


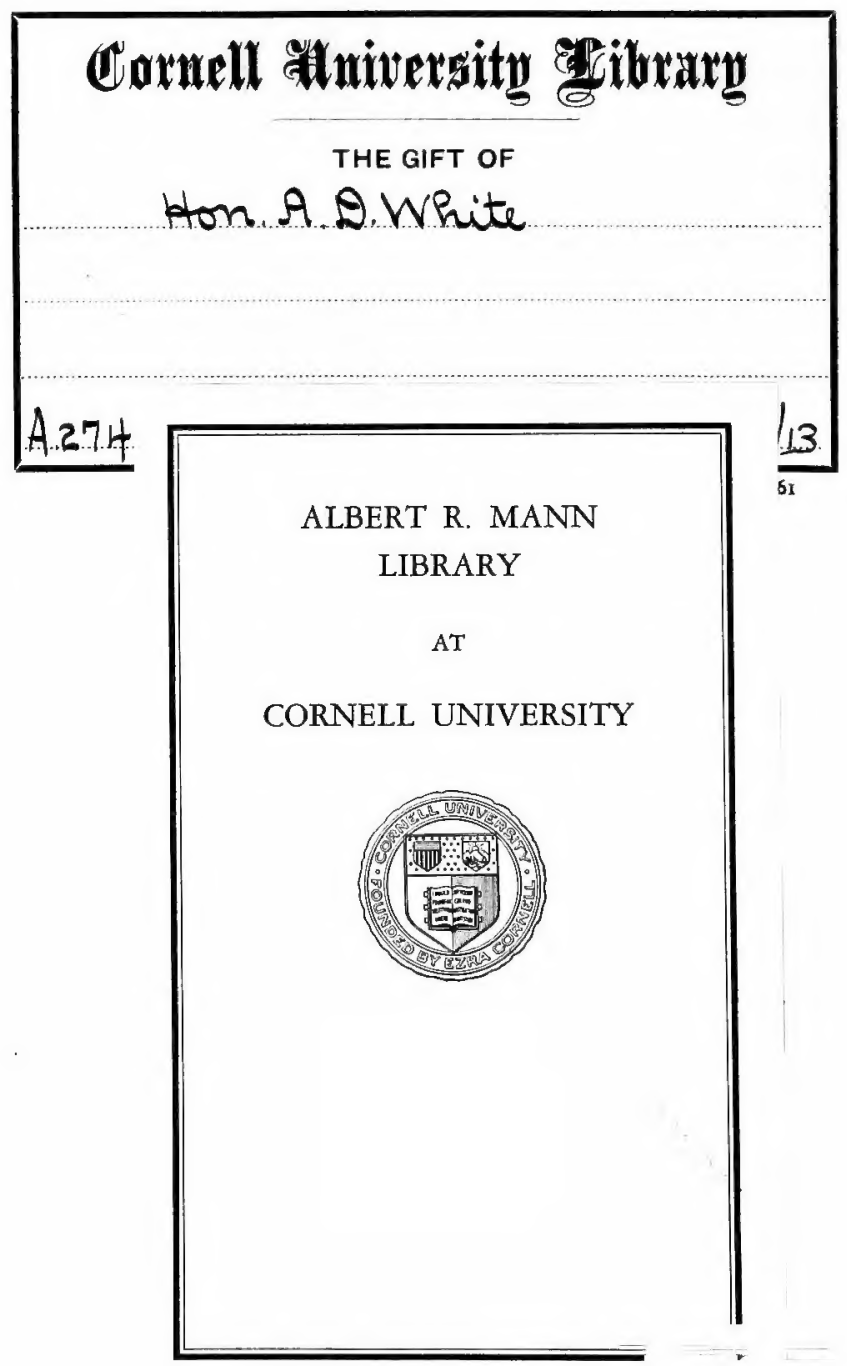




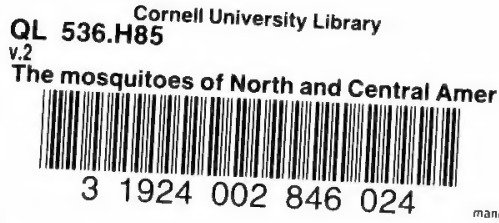




\section{Cornell University Library}

The original of this book is in the Cornell University Library.

There are no known copyright restrictions in the United States on the use of the text. 


\title{
THE MOSQUITOES \\ OF NORTH AND CENTRAL AMERICA AND THE WEST INDIES
}

\author{
BY \\ LELAND O. HOWARD, HARRISON G. DYAR, \\ AND FREDERICK KNAB
}

VOLUME TWO

P LATES

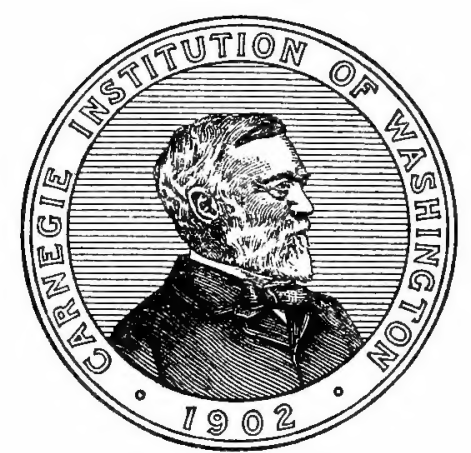

WASHINGTON, D. C.

Published by the Carnegie Institution of Washington 1912 


\section{A.274070 \\ CARNEGIE INSTITUTION OF WASHINGTON \\ Publication No. 159, Vol. Two}

Copies of this Book

mare first issued

FEB 241913

EBe Rord

BALTIMORE, MD., U. B. A. 


\section{EXPLANATION OF PLATES.}

Puate 1. Diagram of Culicid Larva.

PtaATE 2. Male Genitalia: (1) Sabethes cyaneus. (2) Limatus cacophrades. (3) Limatus durhamii. (4) Sabethinus undosus. (5) Limatus methysticus. (6) Sabethinus identicus. (7) Wyeomyia symmachus. (8) Wyeomyia drapetes.

Plate 3. Male Genitalia: (9) Wyeomyia espartana. (10) Wyeomyia codiocampa. (11) Wyeomyia pandora. (12) Wyeomyia bromeliarum. (13) Wyeomyia vanduzeei. (14) Wyeomyia chrysomus. (15) Wyeomyia philophone. (16) Wyeomyia smithii.

Plate 4. Male Genitalia: (17) Wyeomyia matæa. (18) Wyeomyia antoinetta. (19) Wyeomyia abascanta. (20) Wyeomyia mitchellif. (21) Wyeomyia ablabes. (22) Wyeomyia abebela. (23) Wyeomyia galoa. (24) Wyeomyia telestica. (25) Wyeomyia chresta.

PuAte 5. Male Genitalia: (26) Wyeomyia abrachys. (27) Wyeomyia chalcocephala. (28) Wyeomyia simmsi. (29) Wyeomyia leucopisthepus. (30) Wyeomyia scotinomus. (31) Wyeomyia aporonoma. (32) Wyeomyia pseudopecten. (33) Wyeomyia onidus. (34) Wyeomyia pantoia.

Puate 6. Male Genitalia: (35) Wyeomyia cacodela. (36) Wyeomyia eloisa. (37) Wyeomyia circumcincta. (38) Wyeomyia cœnonus. (39) Wyeomyia autocratica. (40) Wyeomyia trinidadensis. (41) Joblotia trichorryes.

PLAte 7. Male Genitalia: (42) Joblotia digitatus. (43) Lesticocampa dicellaphora. (44) Lesticocampa lampropus. (45) Lesticocampa rapax. (46) Lesticocampa longipes. (47) Lesticocampa leucopus. (48) Lesticocampa culicivora. (49) Dinanamesus spanius.

PLAte 8. Male Genitalia: (50) Deinocerites troglodytus. (51) Deinocerites pseudes. (52) Deinocerites cancer. (53) Dinomimetes epitedeus. (54) Culex latisquama. (55) Culex bisulcatus. (56) Culex imitator.

Plate 9. Male Genitalia: (57) Culex inimitabilis. (58) Culex rejector. (59) Culex ocellatus. (60) Culex duplicator. (61) Culex agitator. (62) Culex consternator. (63) Culex daumastocampa. (64) Lutzia bigoti.

Puste 10. Male Genitalia: (65) Carollia urichii. (66) Carollia iridescens. (67) Culex peccator. (68) Culex mutator. (69) Culex abominator. (70) Culex conspirator. (71) Culex incriminator.

Puate 11. Male Genitalia: (72) Culex trachycampa. (73) Culex falsificator. (74) Culex caudelli. (75) Culex conservator. (76) Culex elevator. (77) Culex reductor. (78) Culex chrysonotum. (79) Culex hesitator.

Plate 12. Male Genitalia: (80) Culex pilosus. (81) Culex invocator. (82) Culex deceptor. (83) Culex inhibitator. (84) Culex leprincei. (85) Culex carcinophilus. (86) Culex pleuristriatus. (87) Culex consolator.

Plate 13. Male Genitalia: (88) Culex bastagarius. (89) Culex extricator. (90) Culex mastigia. (91) Culex apeteticus. (92) Culex atratus. (93) Culex derivator. (94) Culex territans. (95) Culex eremita.

PLdTe 14. Male Genitalia: (96) Culex habilitator. (97) Culex stenolepis. (98) Culex pinarocampa. (99) Culex dictator. (100) Culex jubilator. (101) Culex elocutilis. (102) Culex reflector. (103) Culex secutor. (104) Culex vindicator. 
Puste 15. Male Genitalia: (105) Culex factor. (106) Culex equivocator. (107) Culex stígmatosoma. (108) Culex eumimetes. (109) Culex mollis. (110) Culex declarator. (111) Culex revocator. (112) Culex interrogator. (113) Culex proclamator.

PuAte 16. Male Genitalia: (114) Culex revelator. (115) Culex erythrothorax. (116) Culex microsquamosus. (117) Culex towerl. (118) Culex lamentator. (119) Culex janitor. (120) Culex carmodyæ.

Plate 17. Male Genitalia: (121) Culex salinarius. (122) The same, basal parts enlarged. (123) Culex pipiens. (124) Culex restuans, basal parts enlarged. (125) Culex tarsalis. (126) Culex coronator. (127) Culex corniger.

Plate 18. Male Genitalia: (128) Culex quinquefasciatus. (129) The same, basal parts enlarged. (130) Culex quinquefasciatus dipseticus, basal parts enlarged. (131) Culex comitatus, basal parts enlarged. (132) Culex proximus. (133) The same, basal parts enlarged. (134) Culex sphinx.

Plate 19. Male Genitalia: (135) Culex similis. (136) The same, basal parts enlarged. (137) Culex dyari. (138) Culex pipiens. (139) The same, basal parts enlarged. (140) Culex melanurus.

Plate 20. Male Genitalla: (141) Psorophora virescens. (142) Psorophora howardii. (143) Psorophora clliata. (144) Psorophora cilipes. (145) Psorophora sæva. (146) Psorophora sayi.

Plate 21. Male Genitalia: (147) Psorophora horridus. (148) Psorophora pazosi. (149) Psorophora posticatus. (150) Psorophora discolor. (151) Psorophora pygmaea. (152) Psorophora insularius.

Plate 22. Male Genitalia: (153) Psorophora floridense. (154) Psorophora Jamaicensis. (155) Psorophora toltecum. (156) Psorophora columbiæ. (157) Psorophora cingulatus. (158) Psorophora cyanescens.

Plate 23. Male Genitalia: (159) Psorophora haruspicus. (160) Psorophora infine. (161) Psorophora signipennis. (162) Stegoconops equinus. (163) Stegoconops albomaculatus. (164) Stegoconops lucifer.

Plate 24. Male Genitalia: (165) Stegoconops capricornii. (166) Aëdes trichurus. (167) Aëdes diantæus. (168) Aëdes impiger. (169) Aëdes pertinax. (170) Aëdes bimaculatus.

Plate 25. Male Genitalia: (171) Aëdes abfitchii. (172) Aëdes abfitchii. (173) Aëdes fitchii. (174) Aëdes curriei. (175) Aëdes stimulans. (176) Aëdes fletcheri.

Plate 26. Male Genitalia: (177) Aëdes auroides. (178) Aëdes campestris. (179) Aëdes riparius. (180) Aëdes hirsuteron. (181) Aëdes lazarensis. (182) Aëdes pullatus.

Plate 27. Male Genitalia: (183) Aëdes æstivalis. (184) Aëdes cantator. (185) Aëdes spencerif. (186) Aëdes idahoensis. (187) Aëdes grossbecki. (188) Aëdes squamiger.

Plate 28. Male Genitalia: (189) Aëdes curriel. (190) Aëdes onondagensis quaylel. (191) Aëdes euedes. (192) Aëdes atlanticus. (193) Aëdes canadensis. (194) Aëdes serratus.

Plate 29. Male Genitalia: (195) Aëdes abserratus. (196) Aëdes aurifer. (197) Aëdes tortilis. (198) Aëdes cuneatus. (199) Aëdes scapularis. (200) Aëdes infirmatus.

Puate 30. Male Genitalia: (201) Aëdes trivittatus. (202) Aëdes scapularis. (203) Aëdes plutocraticus. (204) Aëdes niger. (205) Aëdes tæniorhynchus. (206) Aëdes mediovittata.

Plate 31. Male Genitalia: (207) Aëdes triseriatus. (208) Aëdes dupreei. (209) Aëdes varipalpus. (210) Aëdes atropalpus. (211) Aëdes sollicitans. (212) Aëdes mitchellæ. 
Plate 32. Male Genitalia: (213) Aëdes nigromaculis. (214) Aëdes epactius. (215) Aëdes fluviatilis. (216) Aëdes podographicus. (217) Aëdes thorntoni. (218) Aëdes aureostriata.

Plate 33. Male Genitalia: (219) Aëdes albonotata. (220) Aëdes busckii. (221) Aëdes oswaldi. (222) Aëdes fulvithorax. (223) Aëdes walkeri. (224) Aëdes calopus.

Plate 34. Male Genitalia: (225) Aëdes sylvestris. (226) Aëdes fuscus. (227) Mansonia flaveolus. (228) Mansonia titillans. (229) Mansonia perturbans. (230) Culiseta inornatus.

Plate 35. Male Genitalia: (231) Culiseta impatiens. (232) Culiseta incidens. (233) Culiseta alaskaensis. (234) Culiseta maccrackenæ. (235) Culiseta dugesi. (236) Bancroftia phyllozoa. (237) Bancroftia waverleyi.

Plate 36. Male Genitalia: (238) Bancroftia signifer. (239) Bancroftia fascipes. (240) Megarhinus portoricensis. (241) Megarhinus hypoptes. (242) Megarhinus trinidadensis. (243) Megarhinus rutila. (244) Megarhinus guadeloupensis.

Plate 37. Male Genitalia: (245) Megarhinus septentrionalis. (246) Megarhinus moctezuma. (247) Megarhinus superbus. (248) Uranotænia lowii. (249) Uranotænia socialis. (250) Uranotænia geometrica. (251) Uranotænia basalis.

Plate 38. Male Genitalia: (252) Uranotænia sapphirinus. (253) Uranotænia caloso. mata. (254) Uranotænia typhlosomata. (255) Aëdeomyia squamipennis. (256) Anopheles bellator. (257) Cœlodiazesis barberi. (258) Anopheles argyritarsis.

Plate 39. Male Genitalia: (259) Anopheles malefactor. (260) Anopheles strigimacula. (261) Anopheles apicimacula. (262) Anopheles quadrimaculatus. (263) Anopheles tarsimaculata. (264) Anopheles albimanus.

Plate 40. Male Genitalia: (265) Anopheles pseudopunctipennis. (266) Anopheles grabhamii. (267) Anopheles eiseni. (268) Anopheles punctipennis. (269) Anopheles crucians. (270) Anopheles occidentalis.

Plate 41. Wings of different Species of Anophelines shown to the same scale: (1) Anopheles crucians. (2) A. malefactor. (3) A. vestitipennis. (4) A. apicimacula. (5) A. punctimacula. (6) A. grabhamii. (7) A. punctipennis. (8) A. neivai. (9) A. bellator. (10) Cœlodiazesis barberi. (11) A. strigimacula. (12) A. tarsimaculata. (13) A. franciscanus. (14) A. albimanus. (15) A. occidentalis. (16) A. eiseni. (17) A. mediopunctata. (18) A. pseudopunctipennis. (19) A. argyritarsis. (20) A. atropos. (21) A. walkeri. (22) A. quadrimaculatus.

Plate 42. Larva: Sabethinus undosus.

PLATE 43. Larva: Wyeomyia vanduzeei.

Plate 44. Larva: Wyeomyia aporonoma.

Plate 45. Larva: Wyeomyia antoinetta.

Plate 46. Larva: Wyeomyia smithii.

Plate 47. Larva: Joblotia digitatus.

Plate 48. Larva: Deinocerites cancer.

Plate 49. Larva: Culex dyari.

Plate 50. Larva: Culex territans.

Plate 51. Larva: Culex latisquama.

Plate 52. Larva: Culex melanurus.

Plate 53. Larva: Culex restuans.

Plate 54. Larva: Culex quinquefasciatus.

Plate 55. Larva: Culex salinarius.

Plate 56. Larva: Culex decorator. 
Plate 57. Larva: Psorophora ciliata.

Plate 58. Larva: Psorophora discolor.

Plate 59. Larva: Psorophora columbiæ.

Plate 60. Larva: Aëdes atropalpus.

Plate 61. Larva: Aëdes canadensis.

Plate 62. Larva: Aëdes sollicitans.

Puate 63. Larva: Aëdes stimulans.

Plate 64. Larva: Aëdes fitchii.

Prate 65. Larva: Aëdes abfitehii.

Prate 66. Larva: Aëdes sylvestris.

Phate 67. Larva: Aëdes cantator.

Plate 68. Larva: Aëdes trivittatus.

PLATE 69. Larva: Aëdes grossbecki.

Plate 70. Larva: Aëdes fuscus.

Plate 71. Larva: Aëdes hirsuteron.

Plate 72. Larva: Aëdes aurifer.

PLAte 73. Larva: Aëdes impiger.

PuAte 74. Larva: Aëdes triseriatus.

Plate 75. Larva: Aëdes varipalpus.

PuATe 76. Larva: Aëdes calopus.

Plate 77. Larva: Stegoconops lucifer.

Plate 78. Larva: Culiseta inornatus.

PLATE 79. Larva: Mansonia perturbans.

Plate 80. Larva: Bancroftia signifer, stage III.

PuAte 81. Larva: Bancroftia signifer.

Puate 82. Larva: Megarhinus geptentrionalis.

PLATE 83. Larva: Uranotænia sapphirinus.

Plate 84. Larva: Anopheles punctipennis.

Plate 85. Larva: Colodiazesis barberi.

Plate 86. Details of Larvæ: (271) Sabethinus identicus. (272) Limatus durhamil. (273) Limatus cacophrades.

Plate 87. Details of Larvæ: (274) Wyeomyia philophone. (275) Wyeomyia pandora. (276) Wyeomyia canfieldi. (277) Wyeomyia trinidadensis.

PLATE 88. Details of Larvæ: (278) Wyeomyia sororcula. (279) Wyeomyia mitchellii. (280) Wyeomyia codiocampa. (281) Wyeomyia ablabes.

Plate 89. Details of Larvæ: (282) Wyeomyia labesba. (283) Wyeomyia abebela. (284) Wyeomyia abia. (285) Wyeomyia autocratica.

PrAte 90. Details of Larvæ: (286) Wyeomyia pseudopecten. (287) Wyeomyia eloisa. (288) Wyeomyia pantoia. (289) Wyeomyia onidus.

Plate 91. Details of Larvæ: (290) Wyeomyia cacodela. (291) Wyeomyia chresta. (292) Wyeomyia telestica. (293) Wyeomyia bromeliarum. (294) Wyeomyia abrachys.

PuAte 92. Details of Larvæ: (295) Wyeomyia symmachus. (296) Wyeomyia chalcocephala. (297) Wyeomyia simmsi. (298) Wyeomyia circumcincta.

Plate 93. Details of Larvæ: (299) Wyeomyia hapla. (300) Joblotia trichorryes. (301) Wyeomyia scotinomus. (302) Joblotia mogilasia. (303) Wyeomyia hemisagnosta.

Plate 94. Details of Larvæ: (304) Lesticocampa rapax. (305) Lesticocampa dicellaphora. (306) Lesticocampa culicivora.

Plate 95. Details of Larvæ: (307) Deinocerites melanophylum. (308) Deinocerites pseudes. (309) Dinomimetes epitedeus. (310) Deinocerites troglodytus.

Plate 96. Detalls of Larvæ: (311) Culex equivocator. (312) Culex extricator. (313) Culex carmodyæ. (314) Culex jubilator. (315) Culex mollis. 
Plate 97. Details of Larvæ: (316) Culex mortificator. (317) Culex declarator. (318) Culex inquisitor. (319) Culex proclamator. (320) Culex revelator.

Plate 98. Details of Larvæ: (321) Culex dictator. (322) Culex vindicator. (323) Culex reflector. (324) Culex interrogator.

Plate 99. Details of Larvæ: (325) Culex revocator. (326) Culex comitatus. (327) Culex pinarocampa. (328) Culex pipiens.

Plate 100. Detalls of Larvæ: (329) Culex factor. (330) Culex microsquamosus. (331) Culex eremita. (332) Culex habilitator.

Plate 101. Details of Larvæ: (333) Culex caraibeus. (334) Culex proximus. (335) Culex similis. (336) Culex barbarus.

Puate 102. Details of Larvæ: (337) Culex janitor. (338) Culex stigmatosoma. (339) Culex erythrothorax. (340) Culex bisulcatus.

Puate 103. Details of Larvæ: (341) Culex stenolepis. (342) Culex corniger. (343) Culex eumimetes. (344) Culex coronator.

Plate 104. Details of Larvæ: (345) Culex azymus. (346) Culex conspirator. (347) Culex tarsalis. (348) Culex abominator.

Plate 105. Details of Larvæ: (349) Culex erraticus. (350) Culex gravitator. (351) Culex investigator. (352) Culex simulator.

Plate 106. Details of Larvæ: (353) Culex leprincei. (354) Culex elevator. (355) Culex inhibitator. (356) Culex atratus.

PLATE 107. Details of Larvæ: (357) Culex lamentator. (358) Culex toweri. (359) Culex bahamensis. (360) Culex secutor.

PIATE 108. Details of Larvæ: (361) Culex mutator. (362) Culex chrysonotum. (363) Culex trachycampa. (364) Culex bastagarius.

PTate 109. Details of Larvæ: (365) Culex pleuristriatus. (366) Culex daumastocampa. (367) Culex rejector. (368) Culex carcinophilus.

PLATE 110. Details of Larvæ: (369) Culex restrictor. (370) Culex derivator. (371) Culex corrigani. (372) Culex conservator.

Plate 111. Details of Larvæ: (373) Culex jenningsi. (374) Culex consolator. (375) Culex inimitabilis. (376) Culex imitator. (377) Culex ocellatus.

PLate 112. Details of Larvæ: (378) Culex caudelli. (379) Culex pilosus. (380) Culex floridanus. (381) Culex agitator.

PLAte 113. Details of Larvæ: (382) Culex reductor. (383) Carrollia urichii. (384) Carrollia iridescens. (385) Lutzia bigoti.

Plate 114. Details of Larvæ: (386) Psorophora sæva. (387) Psorophora virescens. (388) Psorophora howardii. (389) Psorophora cilipes.

Puat'E 115. Details of Larvæ: (390) Psorophora sayi. (391) Psorophora discrucians. (392) Psorophora toltecum. (393) Psorophora floridense. (394) Psorophora jamaicensis.

Plate 116. Details of Larvæ: (395) Psorophora infine. (396) Psorophora pygmæa. (397) Psorophora signipennis. (398) Psorophora haruspicus. (399) Psorophora insularius. (400) Psorophora cyanescens.

PLATE 117. Details of Larvæ: (401) Aëdes fluviatilis. (402) Aëdes pullatus. (403) Aëdes fletcheri. (404) Aëdes mitchellæ.

Plate 118. Details of Larvæ: (405) Aëdes thorntoni. (406) Aëdes bimaculatus. (407) Aëdes lazarensis. (408) Aëdes sansoni.

Ptate 119. Details of Larvæ: (409) Aëdes oswaldi. (410) Aëdes trichurus. (411) Aëdes epactius. (412) Aëdes cuneatus.

PLAtre 120. Details of Larvæ: (413) Aëdes æstivalis. (414) Aëdes scapularis. (415) Aëdes tortilis. (416) Aëdes bracteatus. 
a)

Plate 121. Details of Larvæ: (417) Aëdes curriei. (418) Aëdes knabi. (419) Aëdes onondagensis quaylei. (420) Aëdes podographicus.

Plate 122. Details of Larvæ: (421) Aëdes euplocamus. (422) Aëdes squamiger. (423) Aëdes niger. (424) Aëdes busckil.

Puate 123. Details of Larvæ: (425) Aëdes fulvithorax. (426) Aëdes spencerii. (427) Aëdes auroides. (428) Aëdes serratus.

Plate 124. Details of Larvæ: (429) Aëdes pertinax. (430) Aëdes albonotata. (431) Aëdes abserratus. (432) Aëdes mediovittata.

Plate 125. Detalls of Larvæ: (433) Aëdes walkeri. (434) Aëdes aureostriata. (435) Aëdes depreei.

Plate 126. Details of Larvæ: (436) Aëdes tormentor. (437) Aëdes atlanticus. (438) Stegoconops capricornii. (439) Stegoconops albomaculatus.

Plate 127. Details of Larvæ: (440) Megarhinus portoricensis. (441) Megarhinus trinidadensis. (442) Megarhinus superbus. (443) Megarhinus guadeloupensis.

Plate 128. Detalls of Larvæ: (444) Megarhinus moctezuma. (445) Megarhinus hypoptes. (446) Mansonia titillans.

PuATE 129. Details of Larvæ: (447) Bancroftia fascipes. (448) Bancroftia phyllozoa. (449) Bancroftia waverleyi. (450) Aëdeomyia squamipennis.

PLATE 130. Larval Float-Hairs: (451) Anopheles punctipennis. (452) Anopheles crucians. (453) Anopheles quadrimaculatus. (454) Anopheles pseudopunctipennis. (455) Anopheles grabhamil. (456) Anopheles strigimacula. (457) Anopheles eiseni. (458) Anopheles malefactor. (459) Anopheles argyritaris. (460) Anopheles albimanus and A. tarsimaculata. (461) Anopheles neivai. (462) Colodiazesis barberi.

Plate 131. Head of Larva, ventral view: (463) Sabethinus undosus. (464) Lesticocampa rapax.

Plate 132. Maxillæ of Larvæ: (465) Limatus durhamii. (466) Wyeomyia trinidad. ensis. (467) Wyeomyia aporonoma. (468) Sabethinus undosus. (469) Wyeomyia sororcula. (470) Wyeomyia mitchelli. (471) Wyeomyia smithii. (472) Wyeomyia autocratica. (473) Wyeomyia pseudopecten. (474) Wyeomyia bromeliarum. (475) Wyeomyia telestica. (476) Wyea myia hemisagnosta. (477) Lesticocampa rapax. (478) Joblotia digltatus.

Plate 133. Maxillæ of Larvæ: (479) Deinocerites cancer. (480) Culex mortificator. (481) Culex corniger. (482) Culex dyari. (483) Culex carmodyæ. (484) Culex factor. (485) Culex caudelli. (486) Culex mutator. (487) Culex pleuristriatus. (488) Lutzia bigoti. (489) Culex rejector. (490) Culex inimitabilis. (491) Carrollia urichii.

Plate 134. Maxillæ of Larvæ: (492) Culiseta incidens. (493) Culiseta inornatus. (494) Psorophora ciliata. (495) Psorophora howardii. (496) Psorophora signipennis. (497) Aëdes cantator. (498) Aëdes lazarensis. (499) Aëdes trivittatus. (500) Aëdes euplocamus. (501) Aëdes atlanticus. (502) Aëdes podographicus. (503) Aëdes mediovittata. (504) Aëdes knabi. (505) Aëdes albonotata. (506) Stegoconops luclfer.

Ptate 135. Maxillæ of Larvæ: (507) Bancroftia fascipes. (508) Bancroftia signifer. (509) Megarhinus septentrionalis. (510) Megarhinus superbus. (511) Uranotænla geometrica. (512) Uranotænia coatzacoalcos. (513) Anopheles eiseni. (514) Anopheles grabhamil. (515) Anopheles punctipennis. (516) Anopheles pseudopunctipennis. (517) Anopheles crucians. (518) Anopheles quadimaculatus. (519) Cœlodiazesis barberi 
Plate 136. Mandibles of Larvæ: (520) Sabethinus undosus. (521) Wyeomyia sororcula. (522) Lesticocampa rapax. (523) Joblotia digitatus. (524) Deinocerites cancer. (525) Culex pleuristriatus. (526) Culex rejector. (527) Culex corniger. (528) Culex divisor.

PLate 137. Mandibles of Larvæ: (529) Lutzia bigoti. (530) Culiseta incidens. (531) Psorophora ciliata. (532) Psorophora signipennis. (533) Aëdes cantator. (534) Bancroftia fascipes. (535) Megarhinus superbus. (536) Megarhinus septentrionalis. (537) Stegoconops lucifer.

Plate 138. Mandibles of Larvæ: (538) Uranotænia coatzacoalcos. (539) Uranotænia geometrica. (540) Anopheles grabhamii. (541) Anopheles eiseni. (542) Anopheles punctipennis. (543) Anopheles pseudopunctipennis. (544) Anopheles crucians. (545) Anopheles quadrimaculatus. (546) Cœlodiazesis barberi.

PLATE 139. Mental Plates of Larvæ: (547) Sabethinus undosus. (548) Limatus durhamii. (549) Wyeomyia trinidadensis. (550) Wyeomyia sororcula. (551) Wyeomyia aporonoma. (552) Wyeomyia mitchellii. (553) Wyeomyia smithii. (554) Wyeomyla autocratica. (555) Wyeomyia pseudopecten. (556) Wyeomyia bromeliarum. (557) Wyeomyia telestica. (558) Wyeomyia hemisagnosta. (559) Lesticocampa rapax. (560) Joblotia digitatus. (561) Lutzia bigoti. (562) Culex tarsalis. (563) Culex corniger. (564) Culex dyari.

Plate 140. Mental Plates of Larvæ: (565) Culex mortificator. (566) Culex carmodyæ. (567) Culex habilitator. (568) Culex declarator. (569) Culex inquisitor. (570) Culex proclamator. (571) Culex coronator. (572) Culex derivator. (573) Culex territans. (574) Culex bahamensis. (575) Culex simulator. (576) Culex bisulcatus. (577) Culex conservator. (578) Culex melanurus. (579) Culex extricator. (580) Culex restuans. (581) Culex barbarus. (582) Culex similis.

Plate 141. Mental Plates of Larvæ: (583) Culex quinquefasciatus. (584) Culex pipiens. (585) Culex factor. (586) Culex salinarius. (587) Culex investigator. (558) Culex erraticus. (589) Culex agitator. (590) Culex caudelli. (591) Culex conspirator. (592) Culex carcinophilus. (593) Culex elevator. (594) Culex interrogator. (595) Culex mutator. (596) Culex restrictor. (597) Culex decorator. (598) Culex imitator. (599) Culex rejector. (600) Culex pleuristriatus. (601) Culex gravitator. (602) Culex inimitabilis. (603) Carrollia urichii. (604) Culiseta incidens. (605) Culiseta inornatus. (606) Psorophora ciliata.

Plate 142. Mental Plates of Larvæ: (607) Psorophora howardii. (608) Psorophora sayi. (609) Psorophora discrucians. (610) Psorophora signipennis. (611) Psorophora discolor. (612) Psorophora columbiæ. (613) Psorophora infine. (614) Psorophora indoctum. (615) Psorophora insularius. (616) Aëdes bimaculatus. (617) Aëdes curriei. (618) Aëdes atropalpus. (619) Aëdes varipalpus. (620) Aëdes canadensis. (621) Aëdes sollicitans. (622) Aëdes mitchellæ. (623) Aëdes tæniorhynchus. (624) Aëdes stimulans. (625) Aëdes fitchi1. (626) Aëdes abfitchii. (627) Aëdes sylvestris.

Plate 143. Mental Plates of Larvæ: (628) Aëdes cantator. (629) Aëdes fuscus. (630) Aëdes lazarensis. (631) Aëdes pullatus. (632) Aëdes hirsuteron. (633) Aëdes auroides. (634) Aëdes trichurus. (635) Aëdes triseriatus. (636) Aëdes aurifer. (637) Aëdes trivittatus. (638) Aëdes dupreei. (639) Aëdes euplocamus. (640) Aëdes serratus. (641) Aëdes atlanticus. (642) Aëdes bracteatus. (643) Aëdes podographicus. (644) Aëdes oswaIdi. (645) Aëdes mediovittata. (646) Aëdes calopus. 
Plate 144. Mental Plates of Larvæ: (647) Aëdes knabi. (648) Aëdes walkeri. (649) Aëdes albonotata. (650) Aëdes busckii. (651) Stegoconops lucifer. (652) Stegoconops equinus. (653) Bancroftia fascipes. (654) Bancroftia signifer. (655) Uranotænia geometrica. (656) Uranotænia coatzacoalcos. (657) Megarhinus septentrionalis. (658) The same, second plate. (659) The same, third plate. (660) Anopheles grabhamii. (661) Anopheles eiseni. (662) Anopheles pseudopunctipennis. (663) Anopheles crucians. (664) Anopheles punctipennis. (665) Anopheles quadrimaculatus. (666) Cœlodiazesis barberi.

Puste 145. Eggs: (667) Aëdes atropalpus. (668) Aëdes calopus. (669) Bancroftia signifer.

Plate 146. Eggs: (670) Psorophora ciliata. (671) Psorophora howardil. (672) Psorophora columbiæ. (673) Psorophora horridus. (674) Psorophora discolor. (675) Psorophora sayi. (676) Aëdes triseriatus. (677) Aëdes sylvestris. (678) Aëdes tæniorhynchus. (679) Aëdes sollicitans. (680) Aëdes infirmatus. (681) Aëdes tormentor. (682) Aëdes canadensis.

PLATE 147. Eggs: (683) Aëdes grossbecki. (684) Aëdes calopus. (685) Mansonia perturbans. (686) Culex pipiens. (687) Culex salinarius. (688) Culex territans. (689) Culex erraticus. (690) Culex restuans. (691) Culiseta inornatus. (692) The same, single egg. (693) Anopheles punctipennis, dorsal view. (694) The same, ventral view. (695) Anopheles quadrimaculatus, ventral view. (696) Anopheles crucians, ventral view.

Plate 148. Pupæ: (697) Wyeomyia smithii. (698) Culex territans. (699) Wyeomyia circumcincta. (700) Culex restuans. (701) Culex pipiens. (702) Aëdes sylvestris.

Plate 149. Pupæ: (703) Aëdes fuscus. (704) Psorophora cillata. (705) Psorophora sayi. (706) Psorophora columbiæ. (707) Bancroftia signifer. (708) Aëdes triseriatus.

PLAte 150. Pupæ: (709) Anopheles punctipennis. (710) Aëdes mediovittata. (711) Uranotænia sapphirinus. (712) Aëdes atropalpus. (713) Aëdes calopus. 


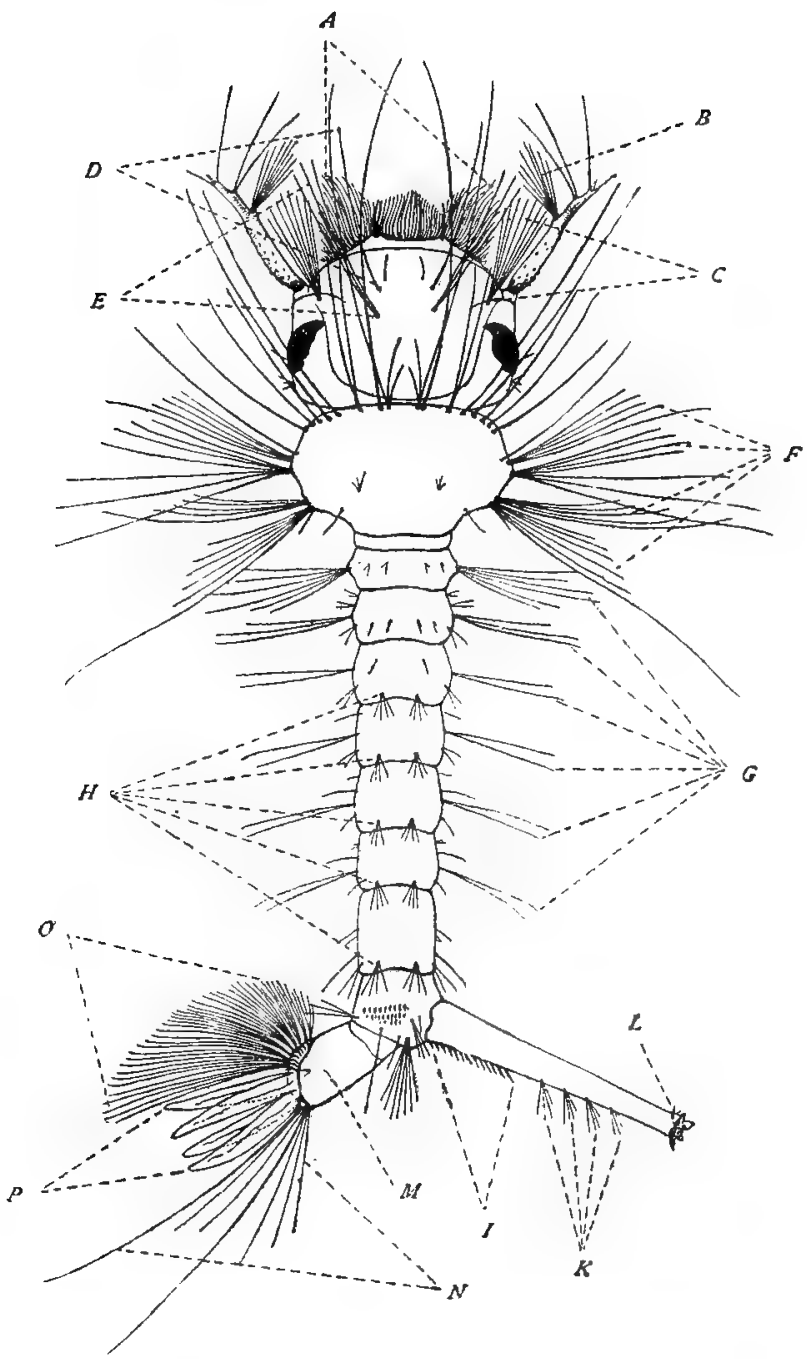

DIAGRAM OF CULICID LARVA.

A. Mouth brushes.

$B$. Tuft of antenna.

C. Ante-antennal tuft.

$D$. Lower frontal tuft.

$E$. Upper frontal tuft.

$F$. Thoracic hair tufts.

$G$. Abdominal lateral tufts.

$H$. Abdominal subdorsal tufts.
I. Pecten of air-tube.

$K$. Hair tufts of air-tube.

$L$. Apical spine of air-tube.

$M$. Anal segment.

$N$. Subdorsal tufts of anal segment.

$O$. Ventral brush.

$P$. Anal gills. 


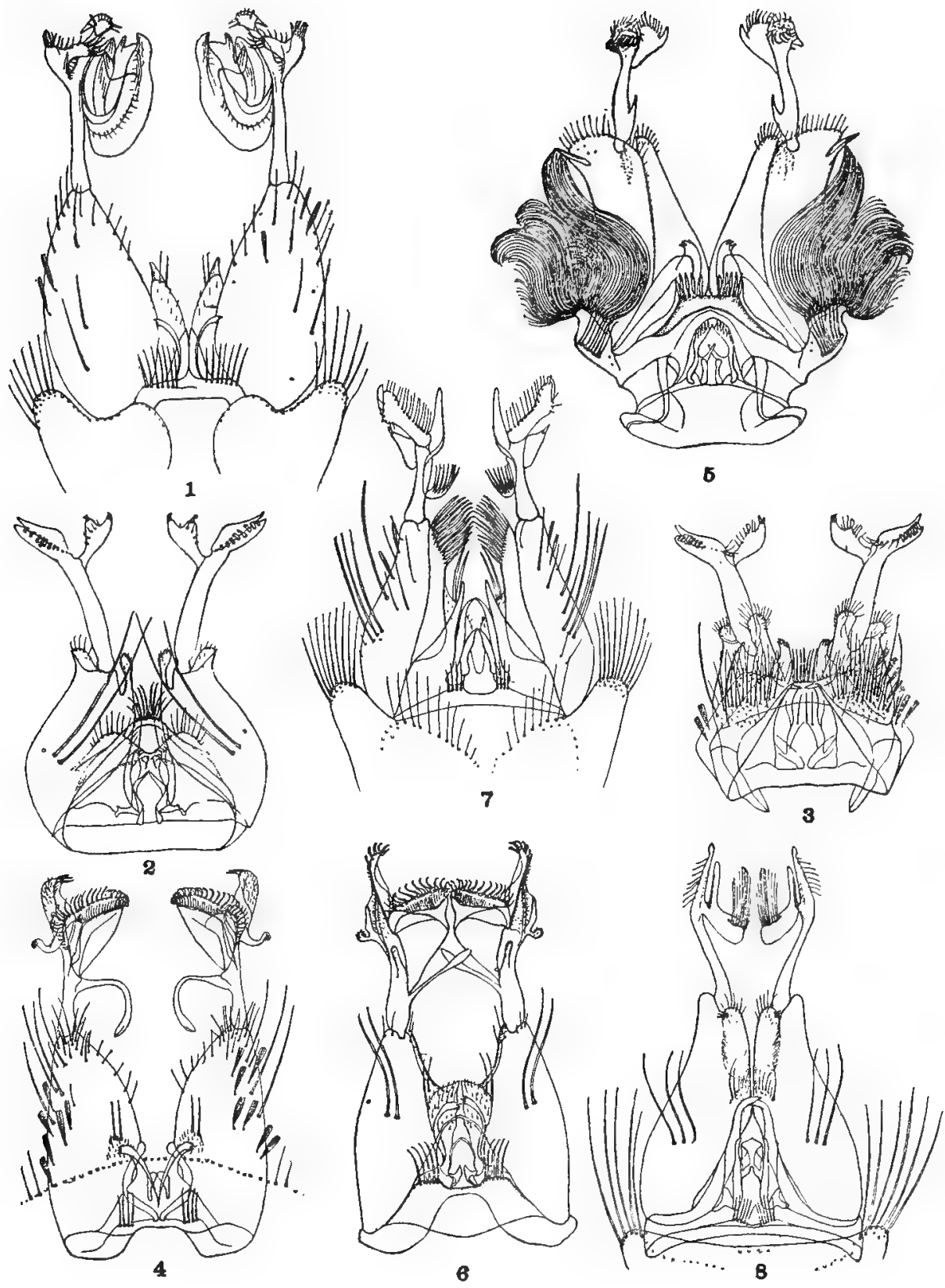

MALE GENI'TALIA.

I. Sabethes cyaneus Fabricius.

2. Limatus cacophrades Dyar \& Knab.

3. Limatus durhamii Theobald.

4. Sabethinus undosus Coquillett.

5. Limatus methysticus Dyar \& Knab.

6. Sabethinus identicus Dyar \& Knab.

7. Wyeomyia symmachus Dyar \& Knab.

8. Wyeomyia drapetes $D$ yar \& $K n a b$. 



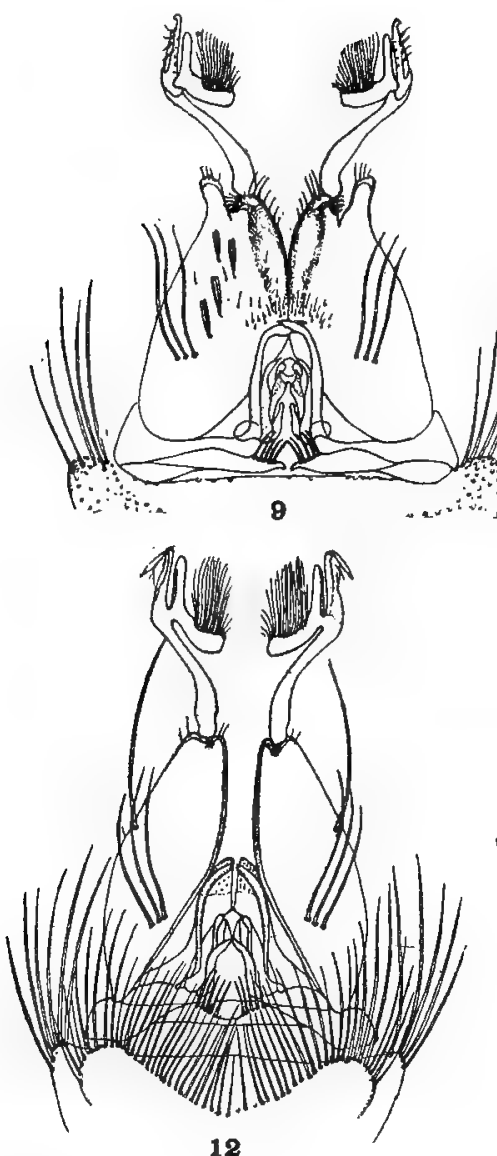

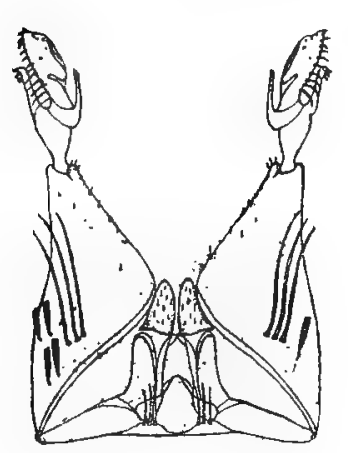

14
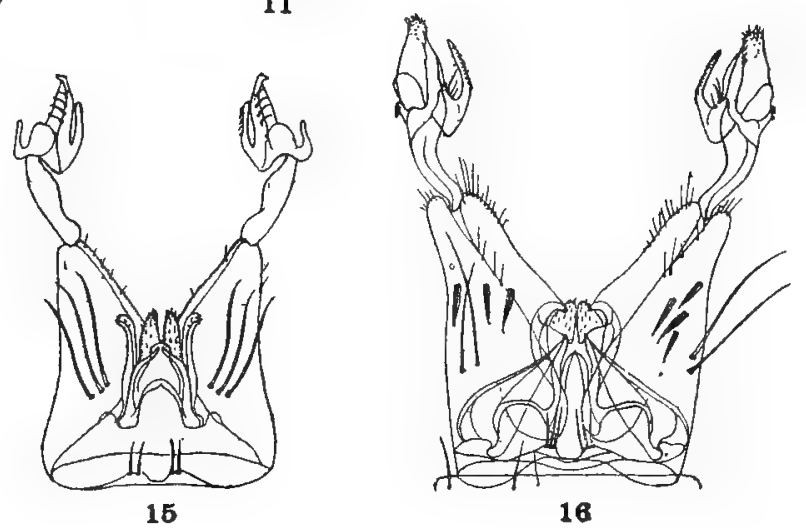

MALE GENITALIA.

9. Wyeomyia espartana Dyar \& Knab.

10. Wyeomyia codiocampa Dyar \& Knab.

II. Wyeomyia pandora Dyar \& Knab.

I2. Wyeomyia bromeliarum Dyar \& Knab.

13. Wyeomyia vanduzeei Dyar \& Knab.

I4. Wyeomyia chrysomus Dyar \& $K n a b$.

I5. Wyeomyia philophone Dyar \& Knab.

I6. Wyeomyia smithii Coquillett. 


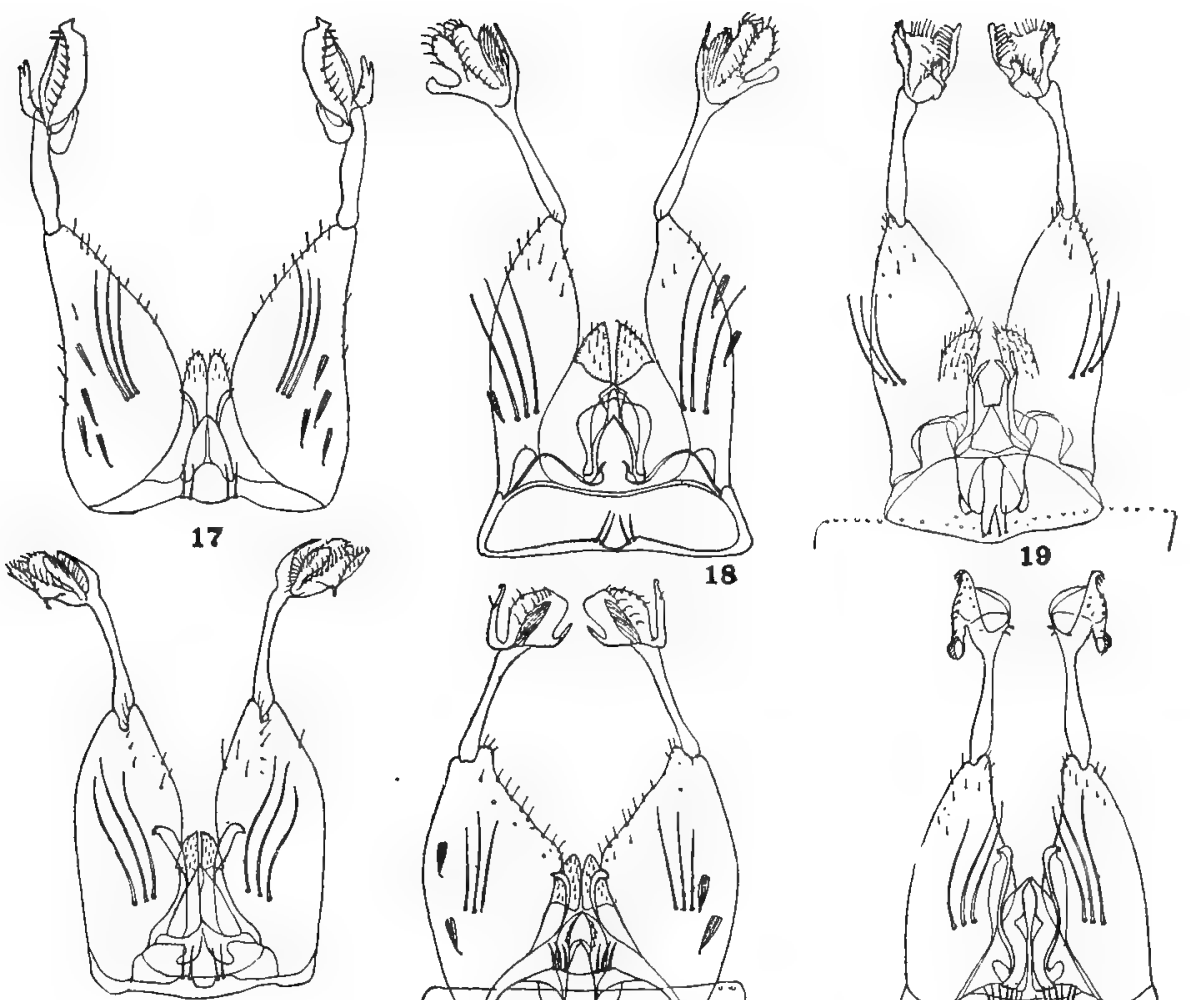

20
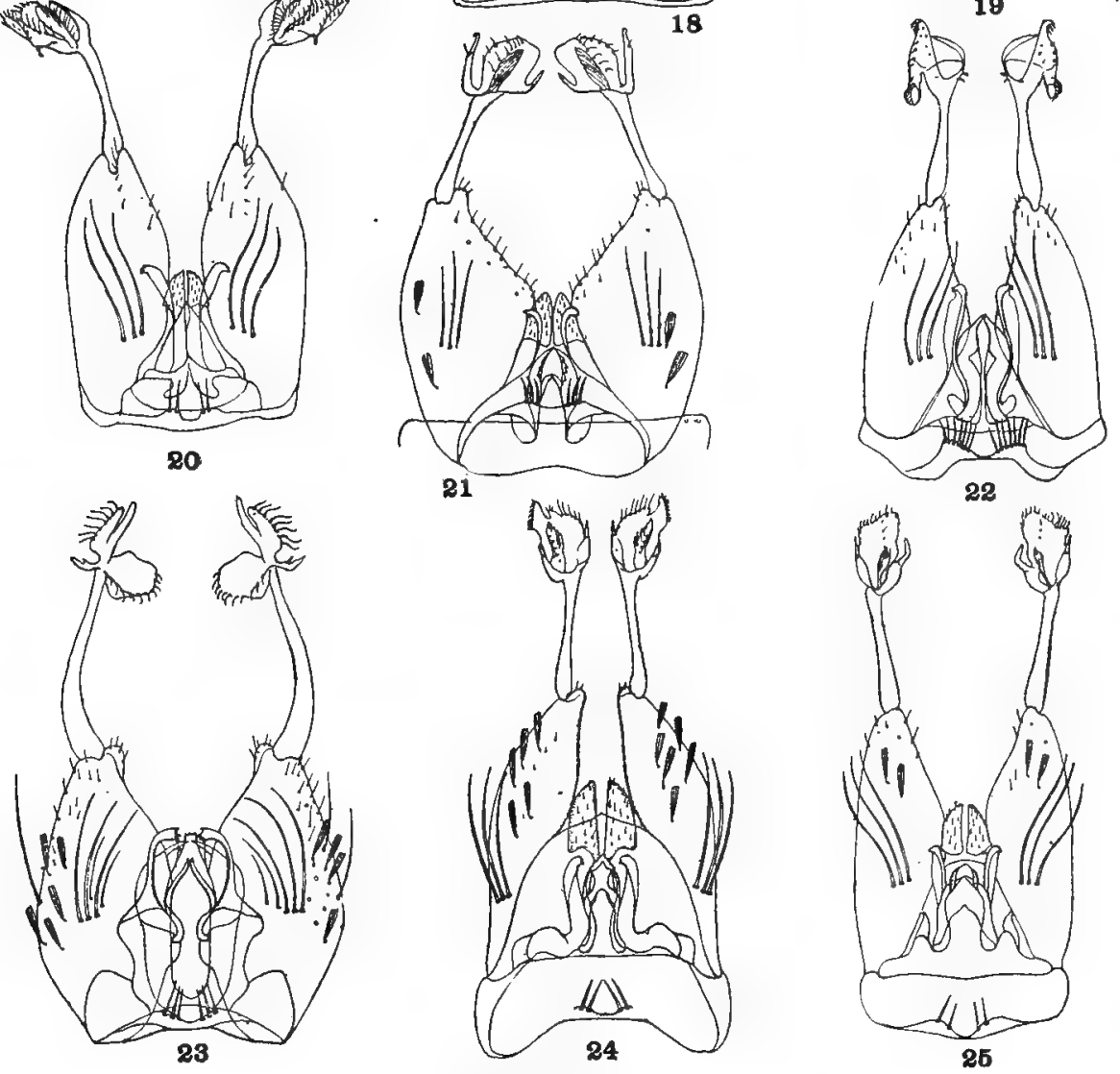

MALE GENITALIA.

17. Wyeomyia matæa Dyar \& Knab.

I8. Wyeomyia antoinetta Dyar \& $K n a b$.

I9. Wyeomyia abascanta Dyar \& $K n a b$.

20. Wyeomyia mitchellii Theobald.

2I. Wyeomyia ablabes Dyar \& Knab.

22. Wyeomyia abebela Dyar $K_{n a b}$.

23. Wyeomyia galoa Dyar \& Knab.

24. Wyeomyia telestica Dyar \& $K$ nab.

25. Wyeomyia chresta Dyar \& $K n a b$. 


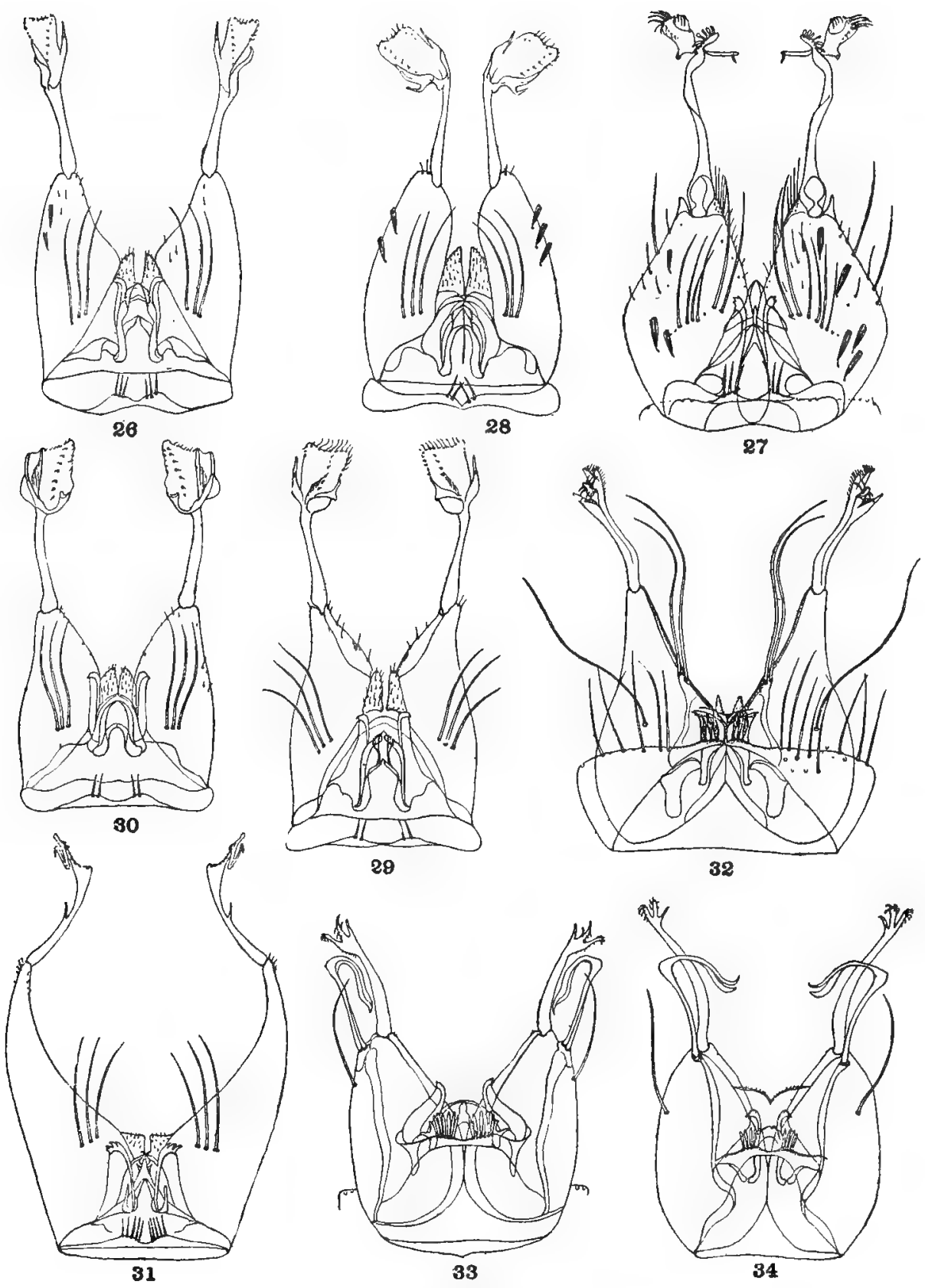

MALE GENITALIA.

26. Wyeomyia abrachys Dyar \& $K n a b$.

27. Wyeomyia chalcocephala Djar \& Knab.

28. Wyeomyia simmsi Dyar \& Knab.

29. Wyeomyia leucopisthepus Dyar \& Knab.

30. Wyeomyia scotinomus Dyar \& Knab.

31. Wyeomyia aporonoma Dyar \& Knab.

32. Wyeomyia pseudopecten Dyar \& Knab.

33. Wyeomyia onidus Dyar \& Knab.

34. Wyeomyia pantoia Dyar \& Knab. 


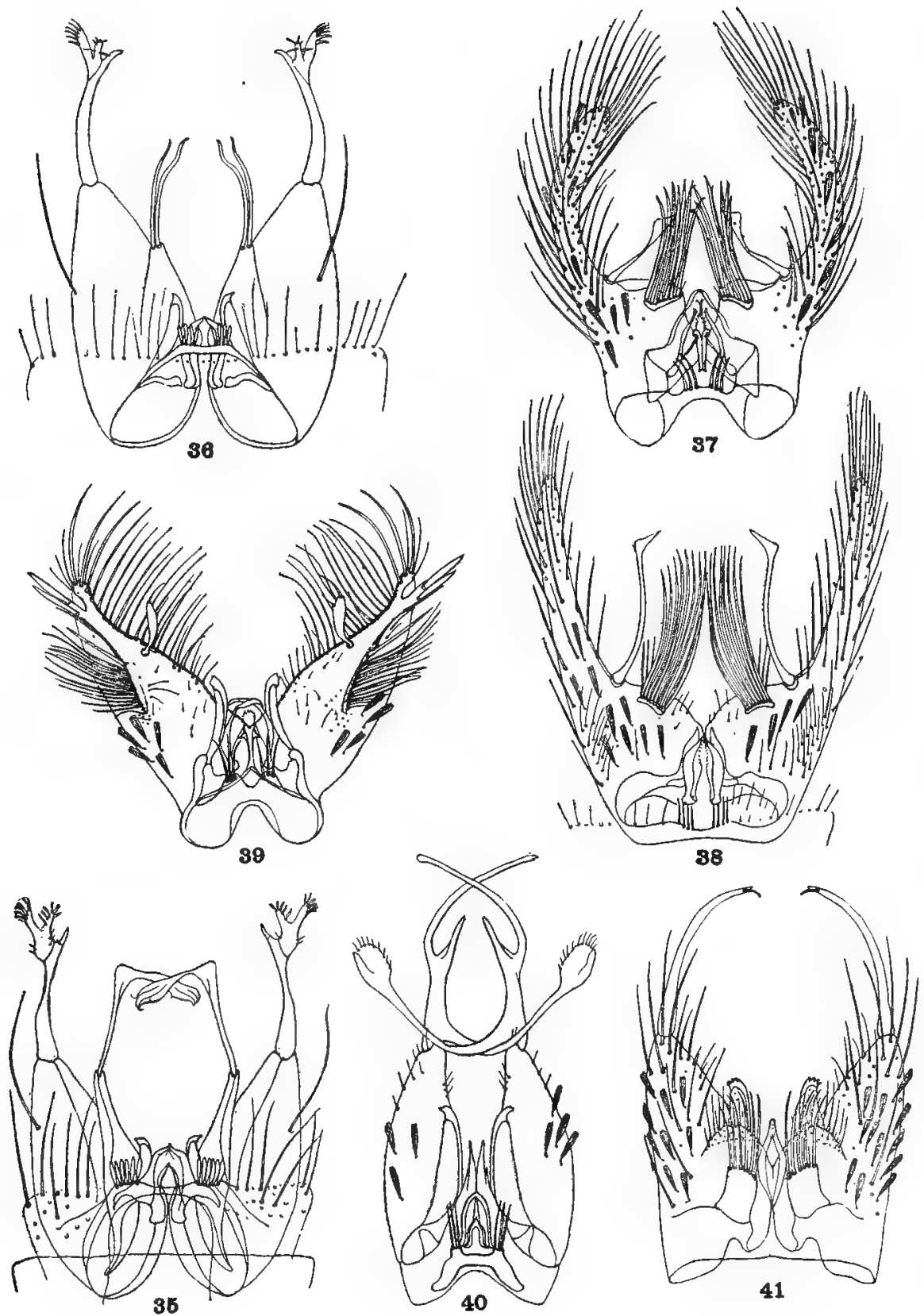

MALE GENITALIA,

35. Wyeomyia cacodela Dyar \& Knab.

36. Wyeomyia eloisa Howard, Dyar \& Knab.

37. Wyeomyia circumcincta Dyar \& Knab.

38. Wyeomyia cœnonus Dyar \& Knab.

39. Wyeomyia autocratica Dyar \& Knab.

40. Wyeomyia trinidadensis Theobald.

4I. Joblotia trichorryes Dyar \& Knab. 


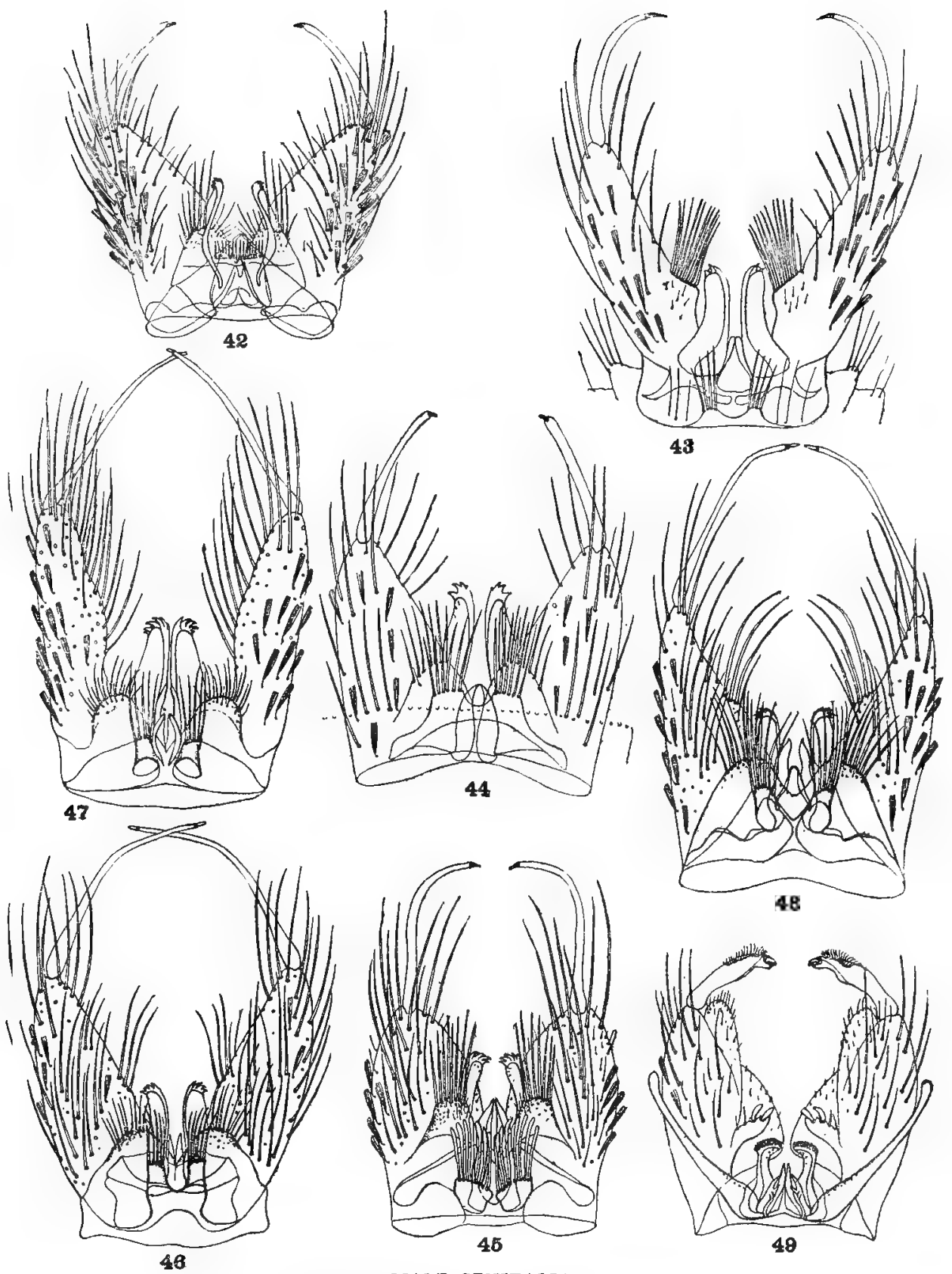

42. Joblotia digitatus Rondani.

43. Lesticocampa dicellaphora Howard, Dyar \& Knab.

44. Lesticocampa lampropus Dyar \& Knab.

45. Lesticocampa rapax Dyar of Knab.

46. Lesticocampa longipes Fabricius.

47. Lesticocampa leucopus $D y a r \& K n a b$.

48. Lesticocampa culicivora Dyar \& Knab.

49. Dinanamesus spanius Dyar \& Knab. 


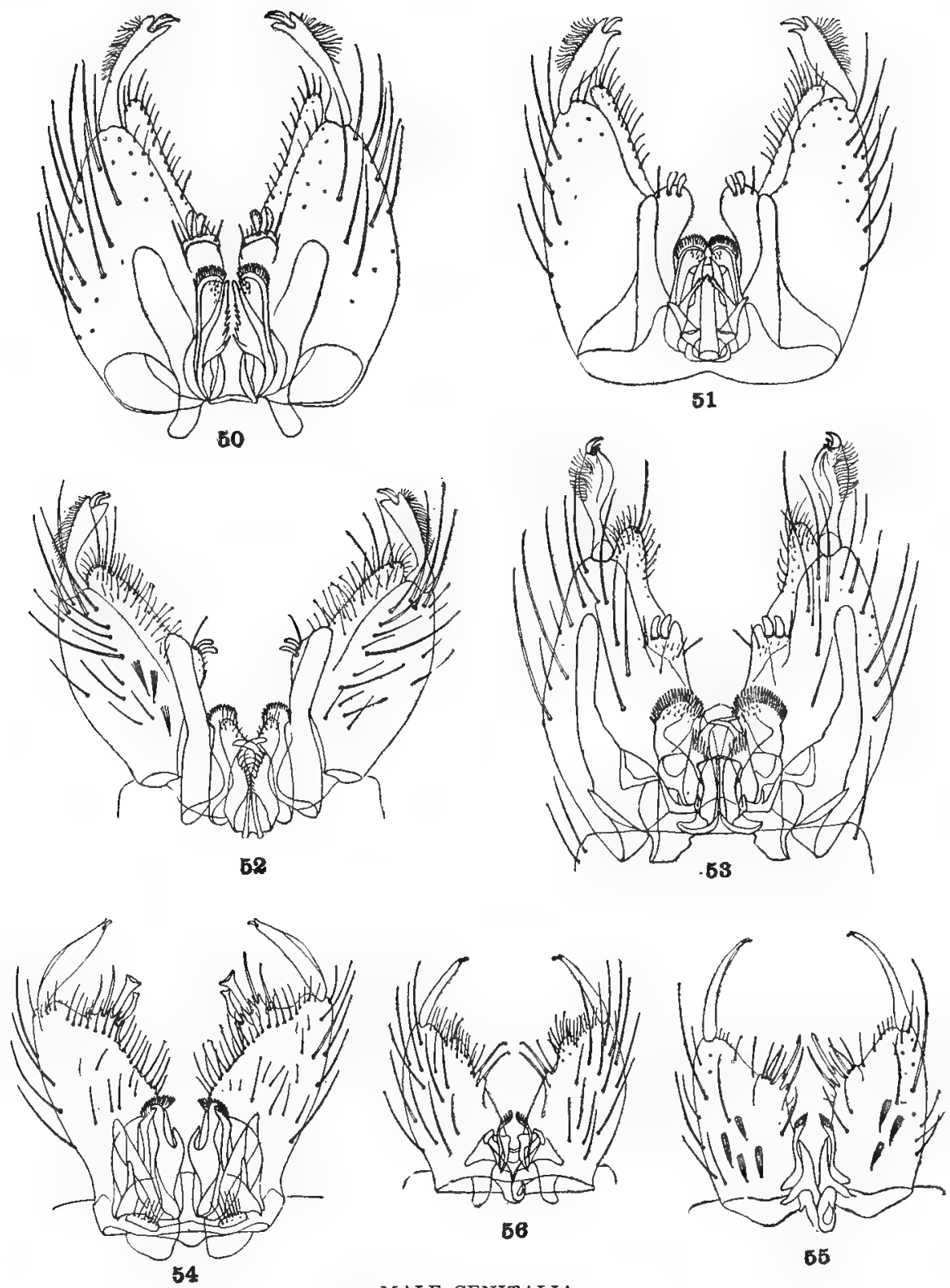

MALE GENITALIA.

50. Deinocerites troglodytus Dyar \& $K n a b$.

51. Deinocerites pseudes Dyar \& Knab.

52. Deinocerites cancer Theobald.

53. Dinomimetes epitedeus $K n a b$.

54. Culex latisquama Coquillett.

55. Culex bisulcatus Coquillett.

56. Culex imitator Theobald. 



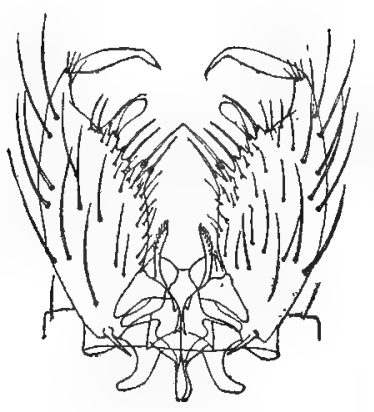

67

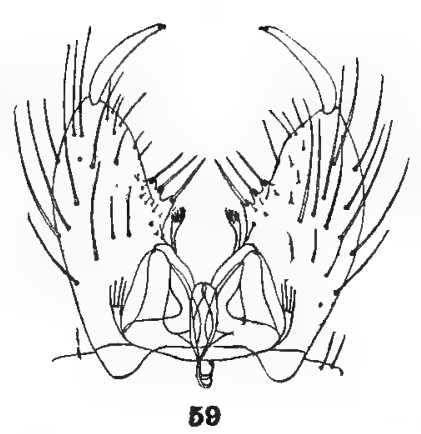

58
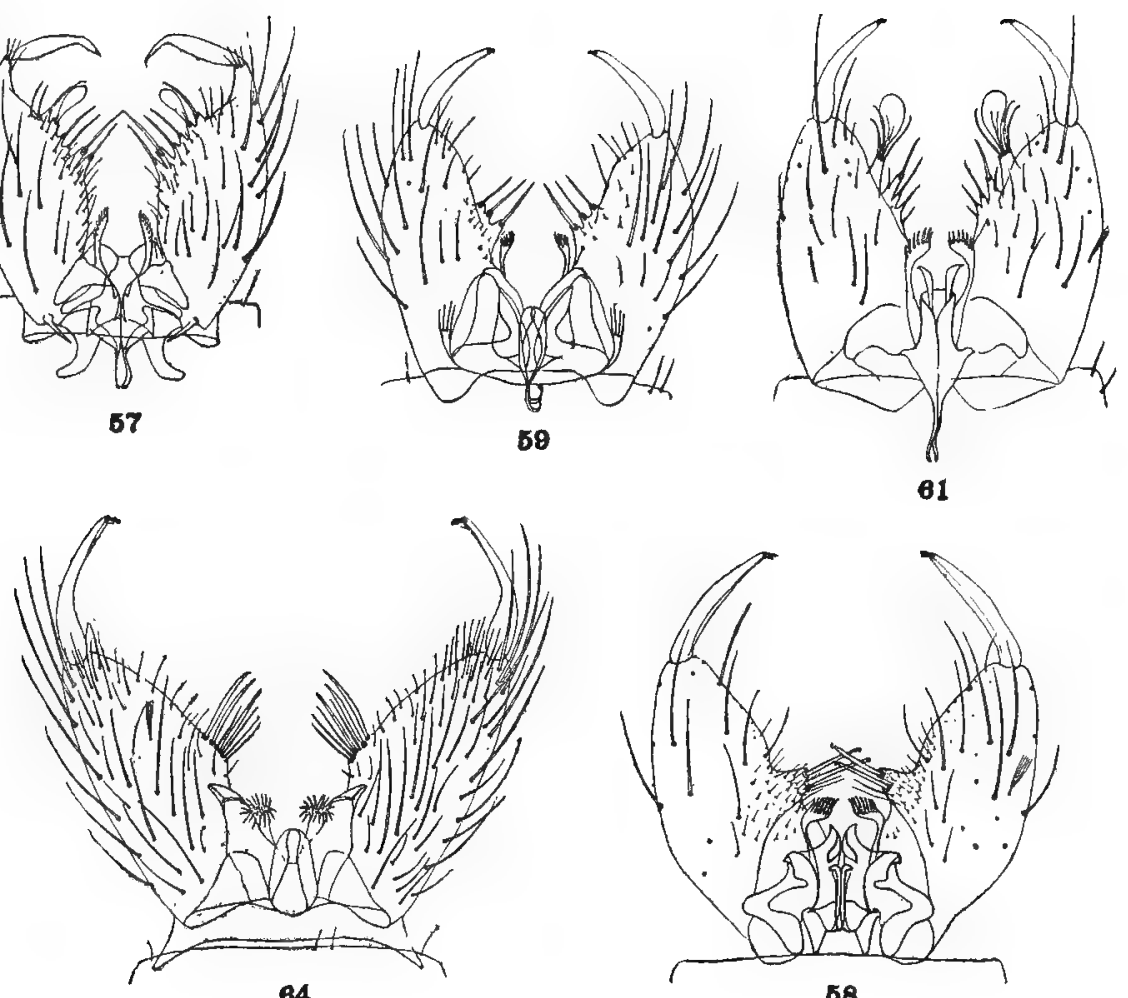

64

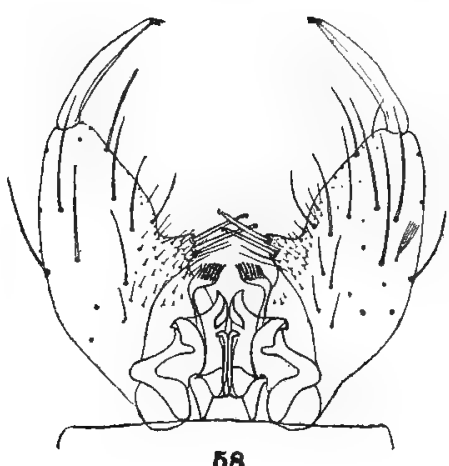

58

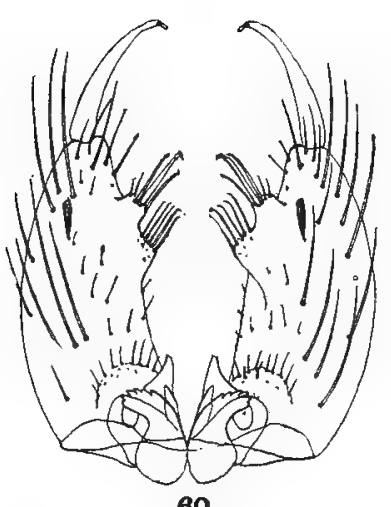

60

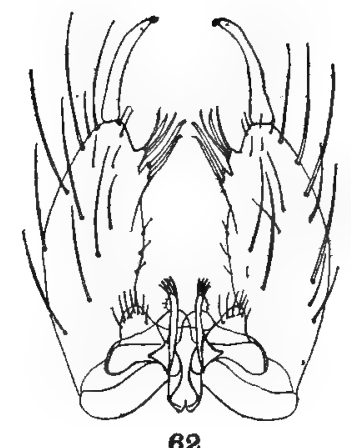

62

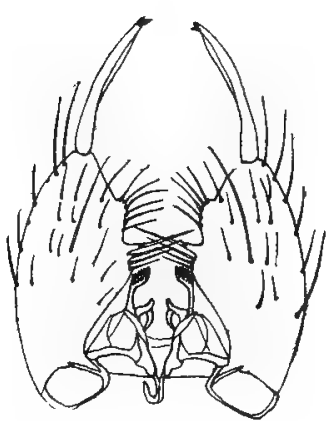

63

MALE GENITALIA.

57. Culex inimitabilis, Dyar \& Knab.

58. Culex rejector Dyar \& $K n a b$.

59. Culex ocellatus Theobald.

60. Culex duplicator Dyar \& Knab.

61. Culex agitator Dyar \& Knab.

62. Culex consternator Dyar \& Knab.

63. Culex daumastocampa Dyar \& Knab.

64. Lutzia bigoti Bellardi. 


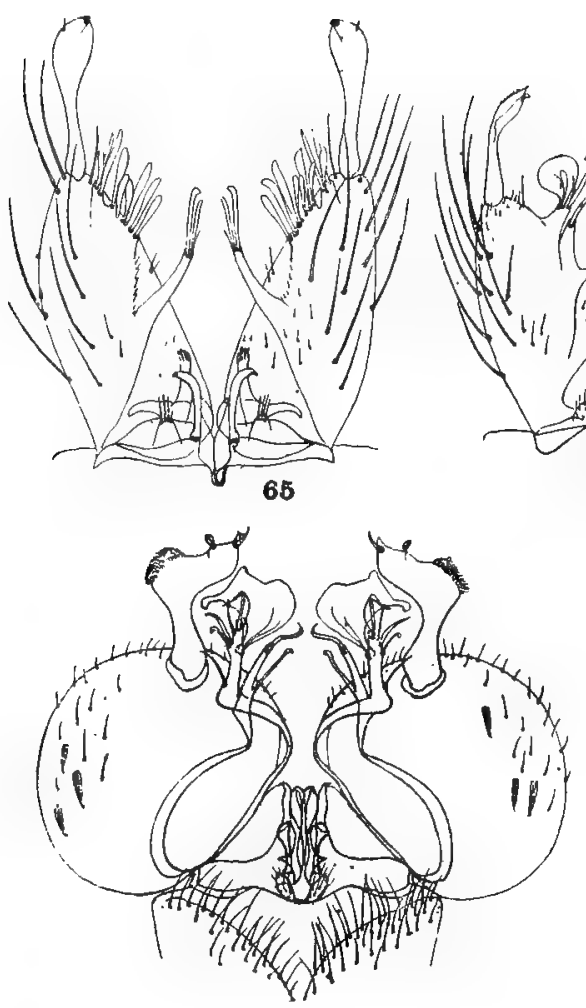

69

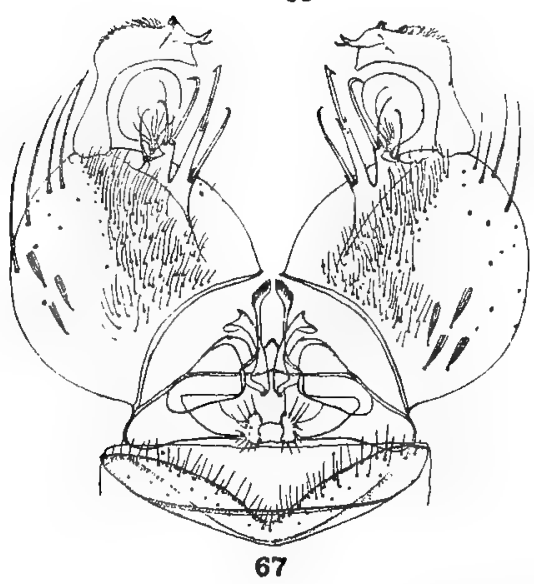

MALE GENITALIA.

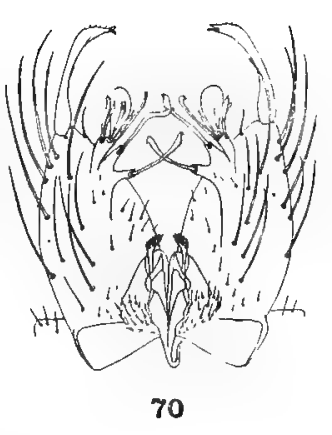

68
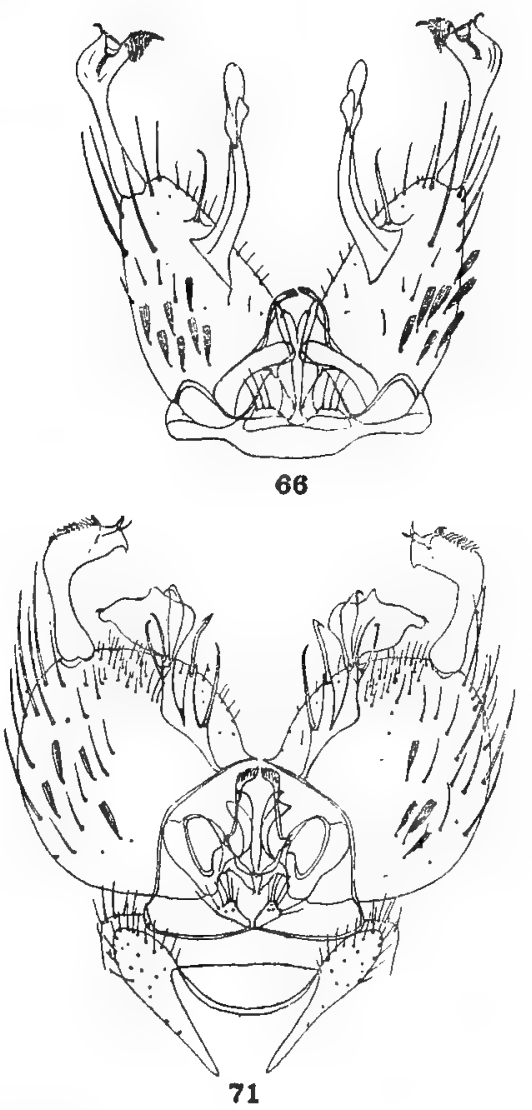

65. Carollia urichii Coquillett.

66. Carrollia iridescens Lutz.

67. Culex peccator Dyar \& Knab.

68. Culex mutator Dyar \& $K n a b$.

69. Culex abominator Dyar \& Knab.

7o. Culex conspirator Dyar \& Knab.

71. Culex incriminator Dyar \& Knab. 



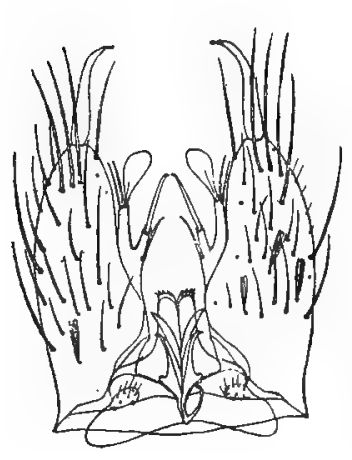

73

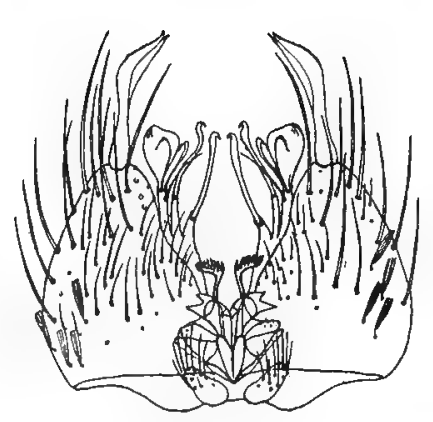

72

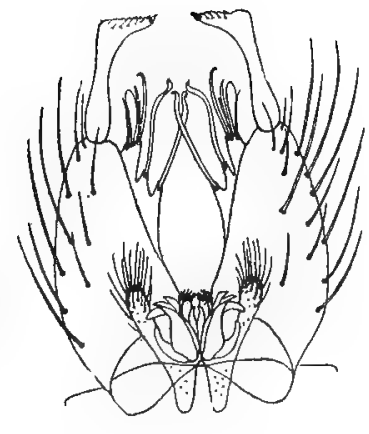

74
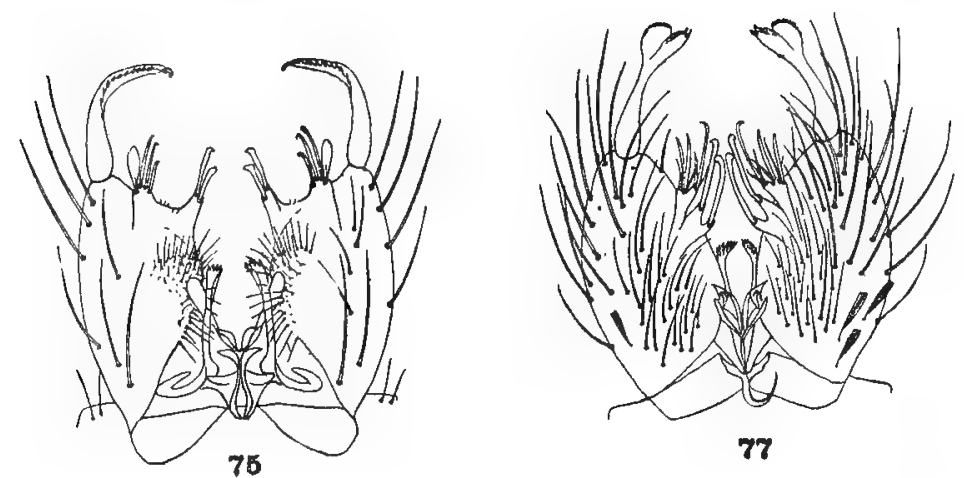

77

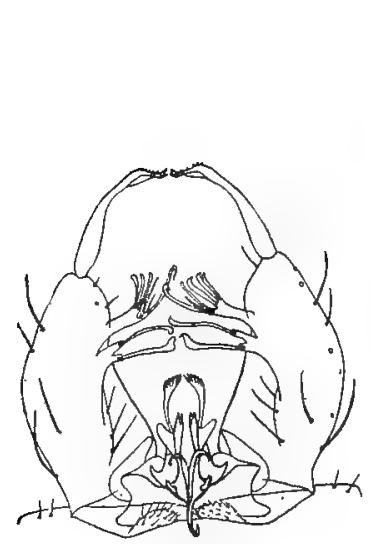

76

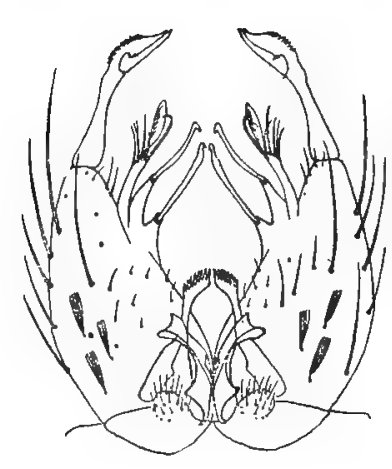

78

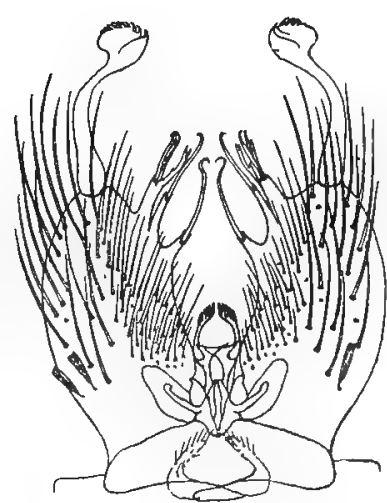

79

MALE GENITALIA.

72. Culex trachycampa $D y a r \& K n a b$.

73. Culex falsificator Dyar \& Knab.

74. Culex caudelli Dyar \& Knab.

75. Culex conservator Dyar $\mathcal{E} K$ Knab.

76. Culex elevator Dyar \& Knab.

77. Culex reductor Dyar \& Knab.

78. Culex chrysonotum Dyar \& Knab.

79. Culex hesitator Dyar \& Knab. 



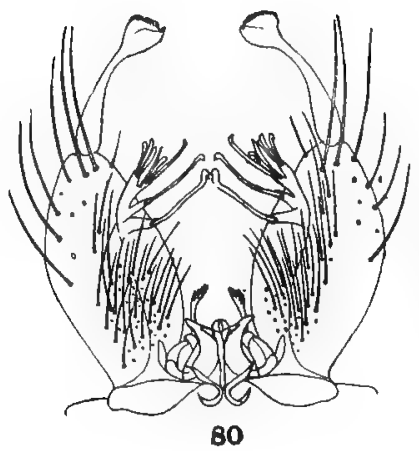

BO

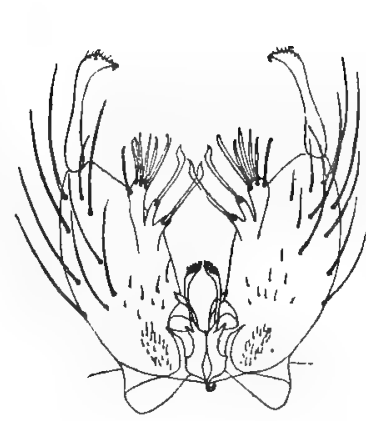

83

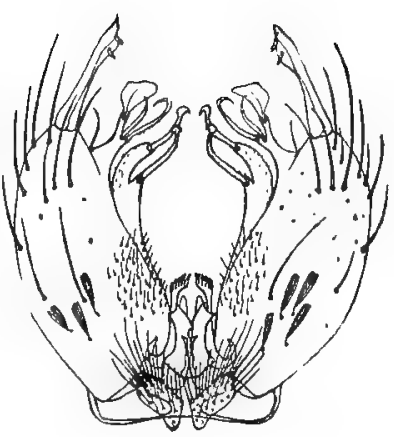

81
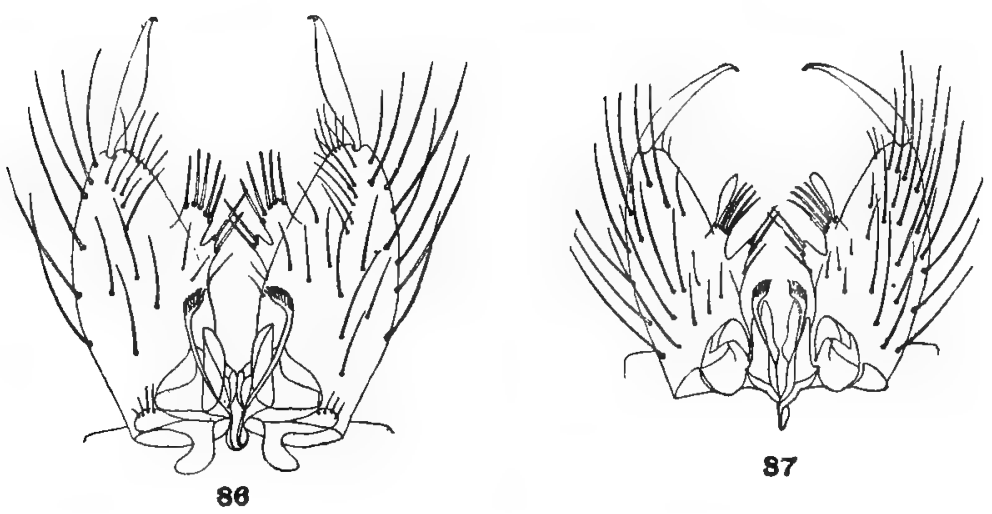

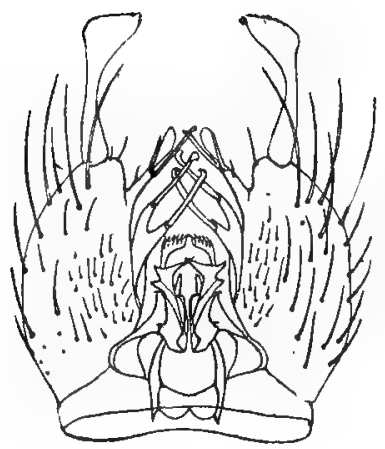

82

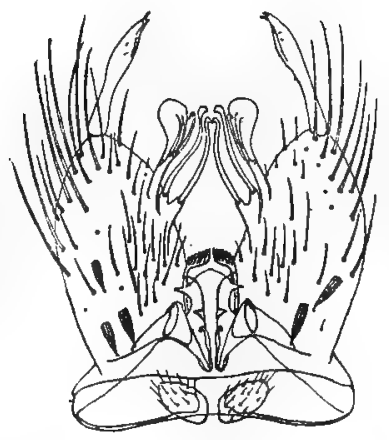

84

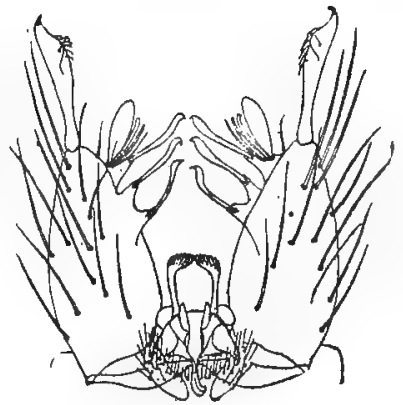

85

MALE GENITALIA.

80. Culex pilosus Dyar \& Knab.

81. Culex invocator Pazos.

82. Culex deceptor Dyar \& Knab.

83. Culex inhibitator Dyar \& Kuab.

84. Culex leprincei Dyar \& Knab.

85. Culex carcinophilus Dyar \& Knab.

86. Culex pleuristriatus Theobald.

87. Culex consolator Dyar \& Knab. 



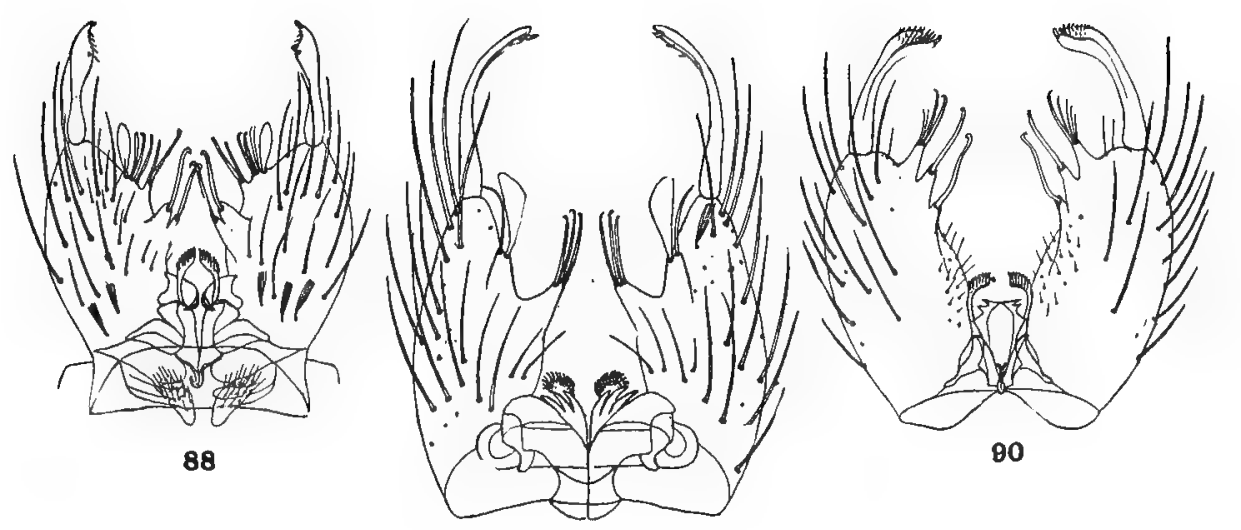

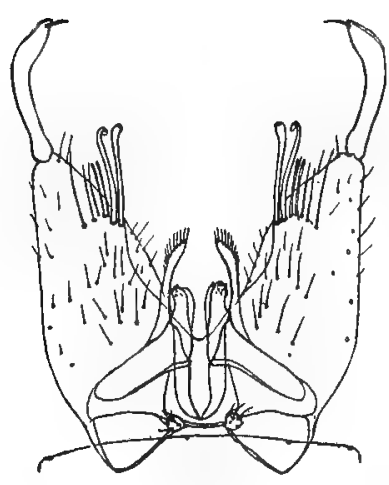

83

89

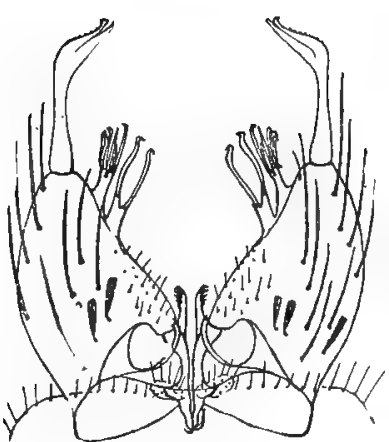

91

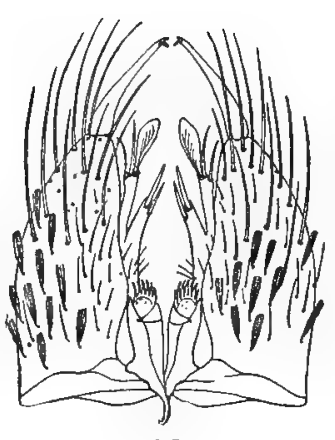

92

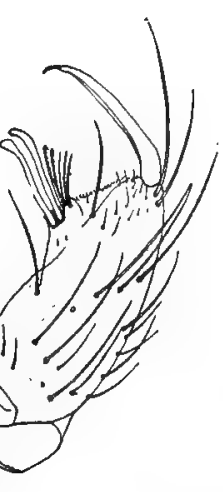

04

MALE GENITALIA.

88. Culex bastagarius Dyar \& Knab.

89. Culex extricator Dyar \& $K n a b$.

90. Culex mastigia Howard, Dyar \& Knab.

9r. Culex apeteticus Howard, Dyar \& Knab.

92. Culex atratus Theobald.

93. Culex derivator Dyar \& Knab.

94. Culex territans $W$ alker

95. Culex eremita Howard, Dyar \& Knab. 


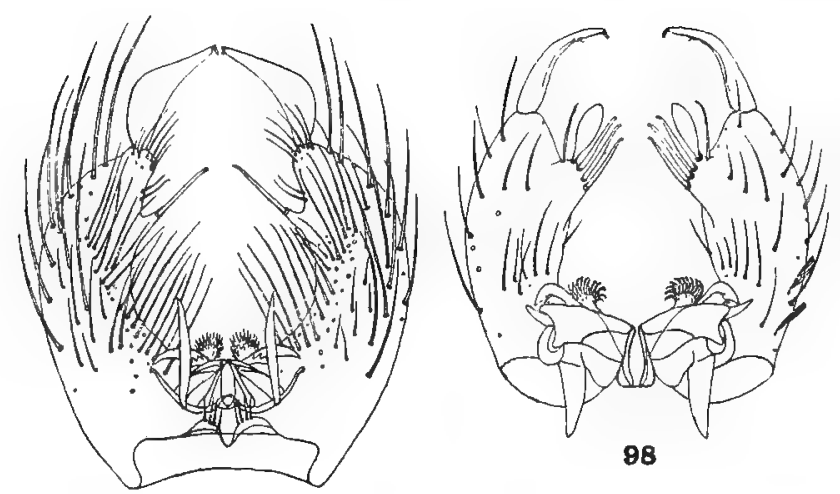

98

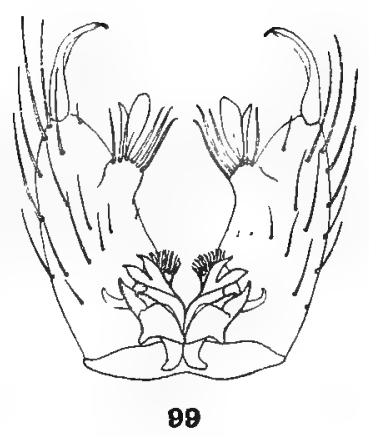

96
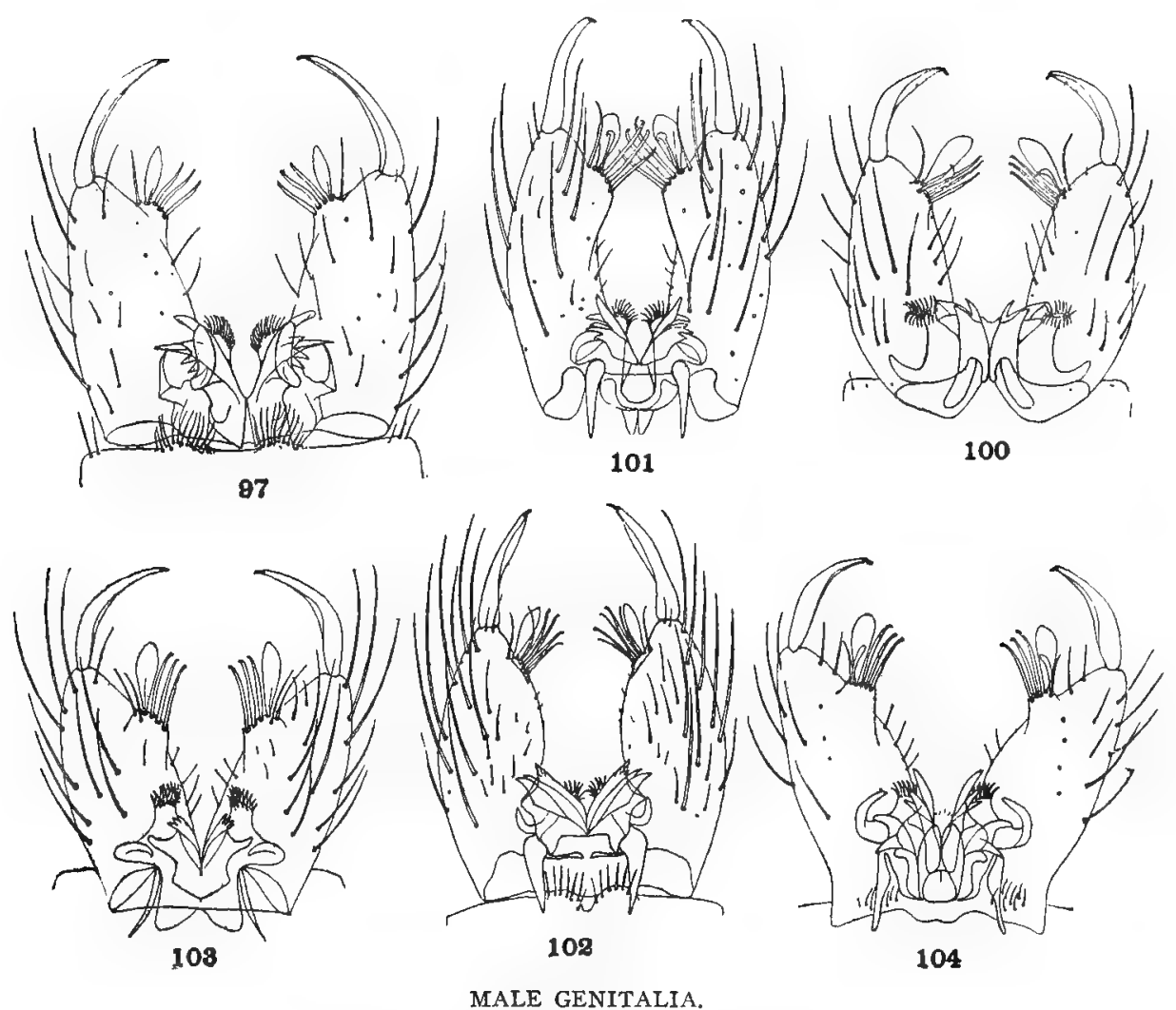

96. Culex habilitator Dyar \& Knab.

97. Culex stenolepis Dyar \& $K n a b$.

98. Culex pinarocampa Dyar \& $K n a b$.

99. Culex dictator Dyar \& Knab.

Ioo. Culex jubilator Dyar \& $K n a b$.

Ior. Culex elocutilis Dyar \& Knab.

102. Culex reflector Dyar \& $K n a b$.

103. Culex secutor Theobald.

104. Culex vindicator Dyar \& Knab. 


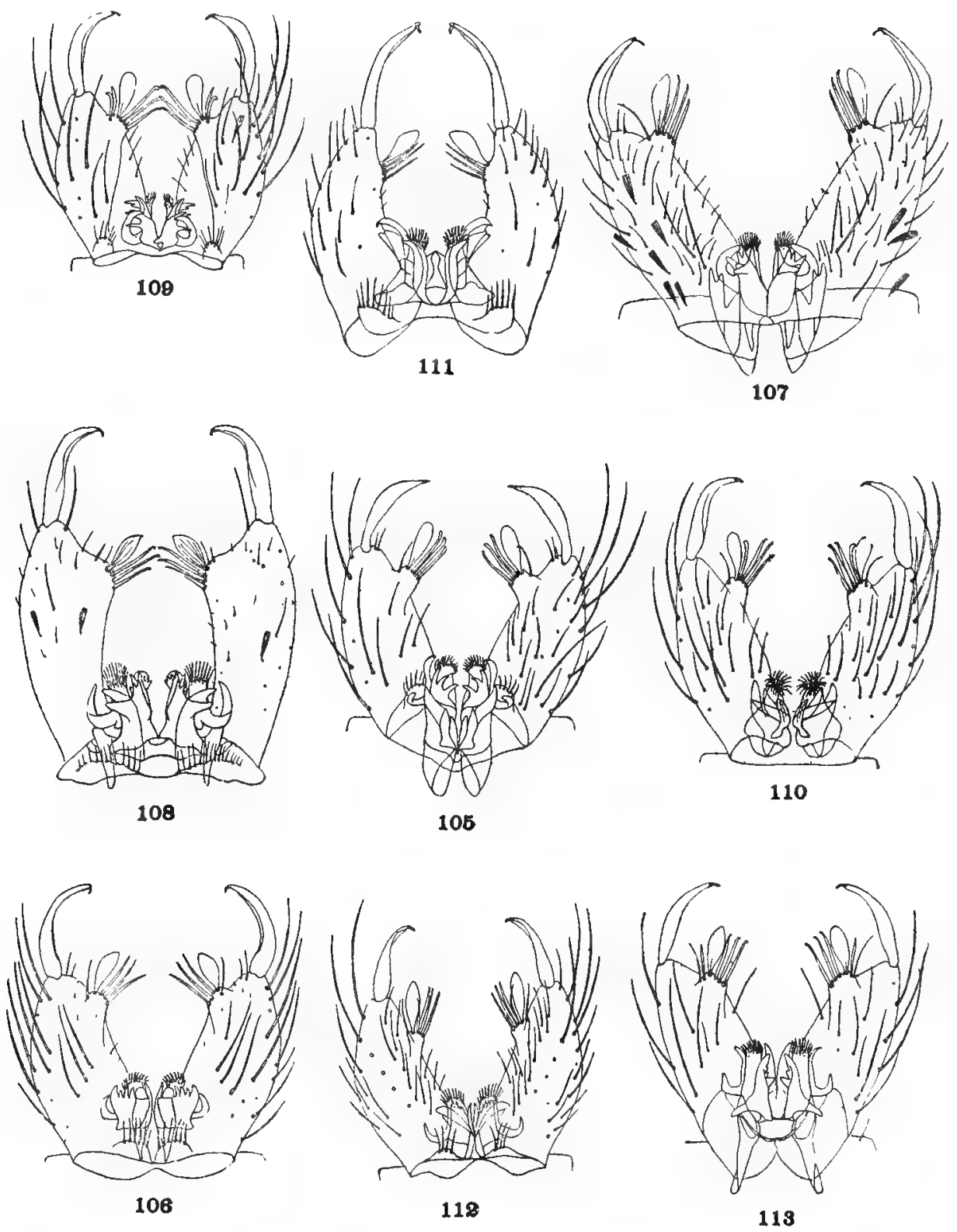

MALE GENITALIA.

105. Culex factor Dyar \& Knab.

Io6. Culex equivocator Dyar \& Knab.

I07. Culex stigmatosoma Dyar.

I08. Culex eumimetes Dyar \& Knab.

I09. Culex mollis Dyar \& $K u a b$.

IIo. Culex declarator Dyar \& Knab.

III. Culex revocator Dyar \& $K n a b$.

I12. Culex interrogator Dyar \& Knab.

ri3. Culex proclamator Dyar \& Knab. 


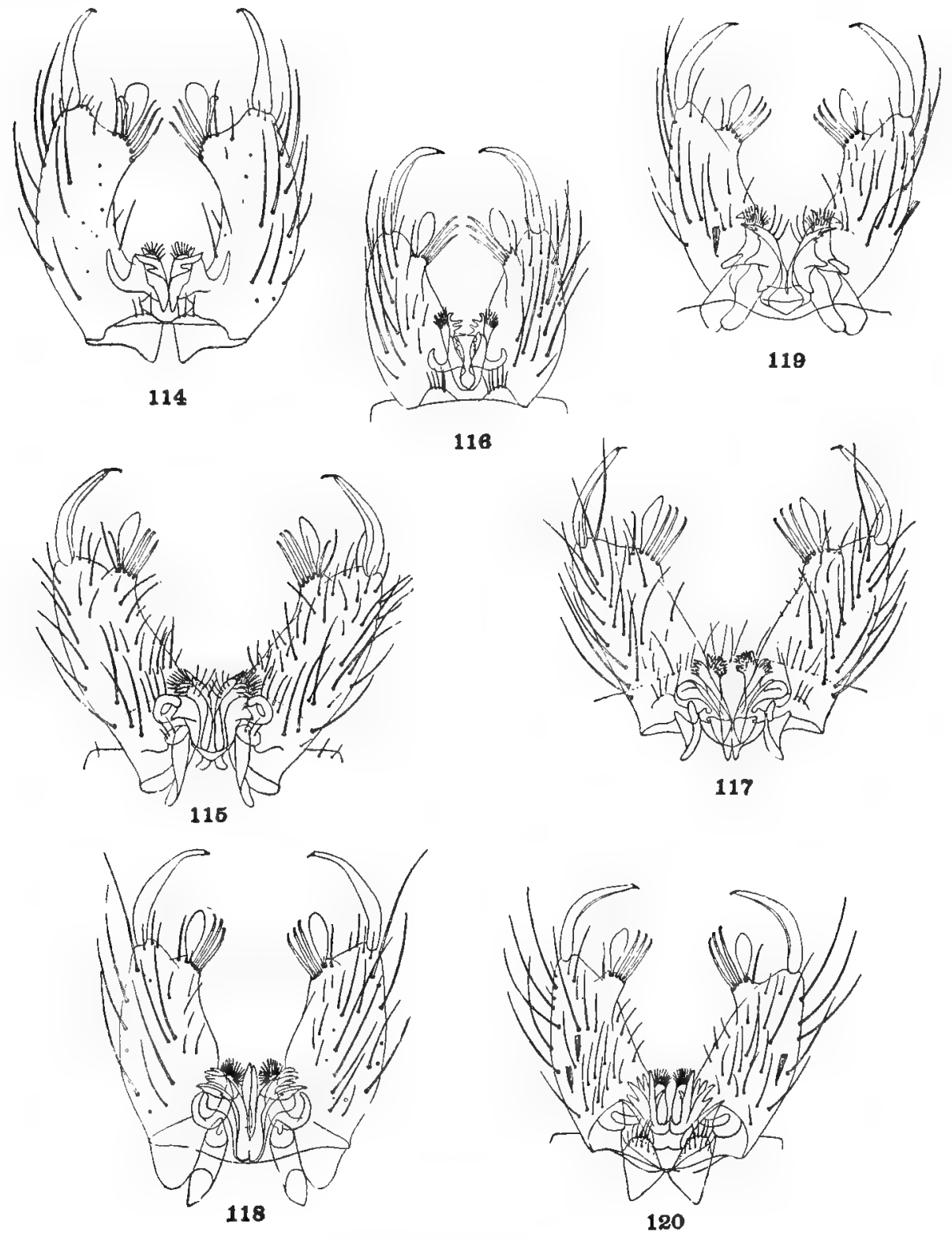

MALE GENITALIA.

I 4. Culex revelator Dyar $\mathcal{E}$ Knab.

II5. Culex erythrothorax Dyar.

II6. Culex microsquamosus Grabham.

II7. Culex toweri Dyar \& $K n a b$.

II8. Culex lamentator Dyar \& Kuab.

II9. Culex janitor Theobald.

120. Culex carmodyæ Dyar \& Knab. 


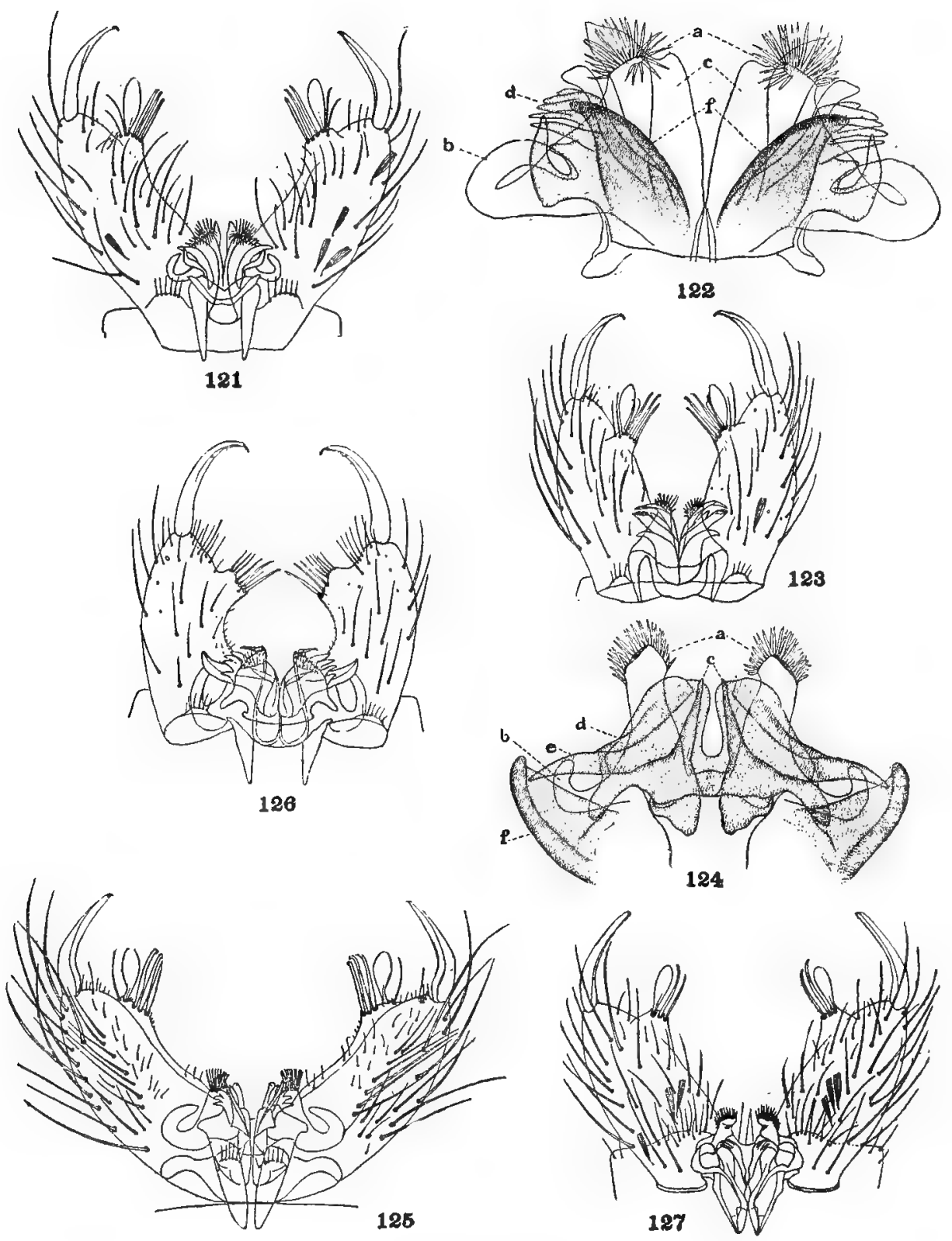

MALE GENITALIA.

12I. Culex salinarius Coquillett.

122. The same, basal parts enlarged.

123. Culex pipiens Linneus.

124. Culex restuans Theobald, basal parts enlarged.

125. Culex tarsalis Coquillett.

126. Culex coronator Dyar \& Knab.

127. Culex corniger Theobald. 


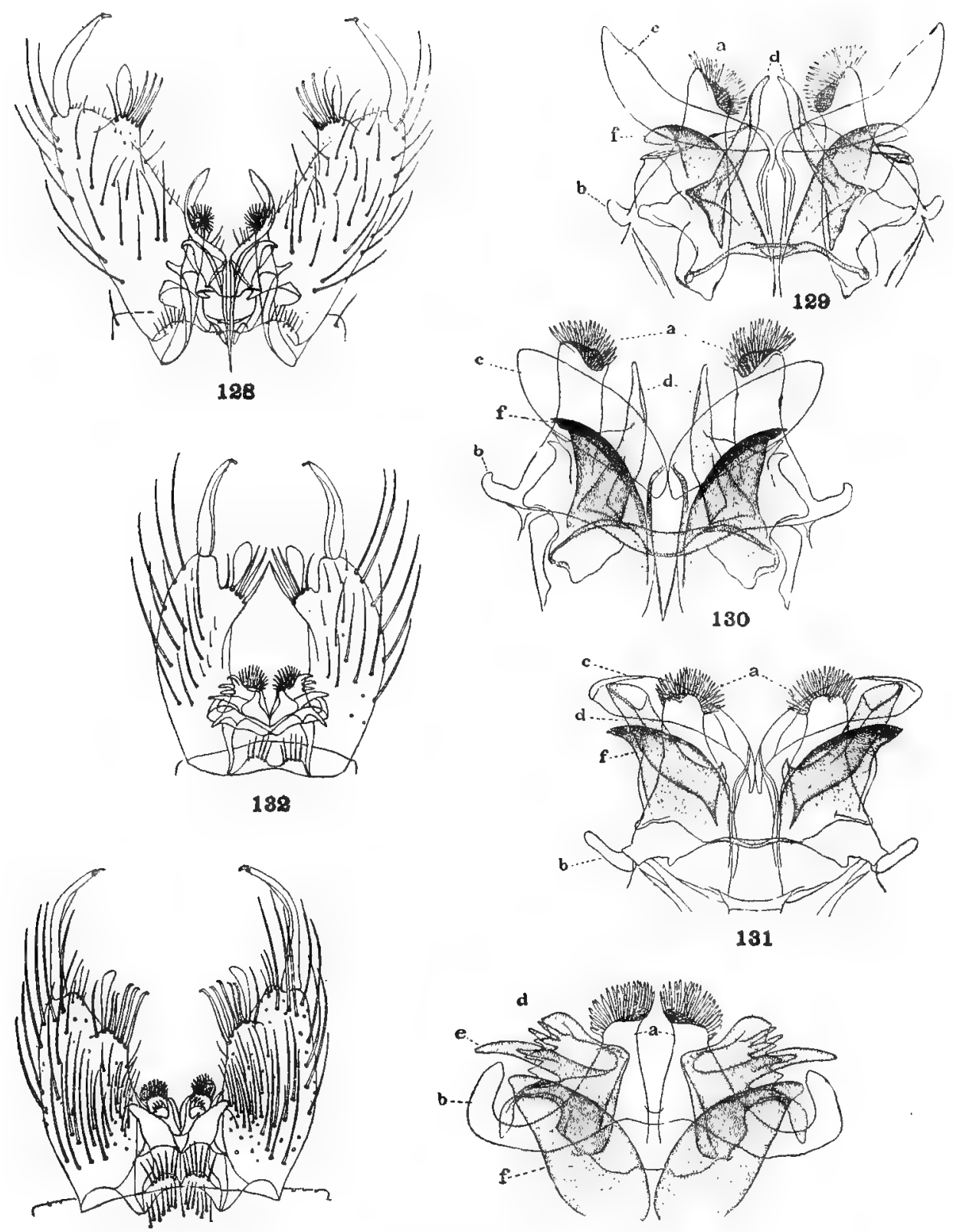

134

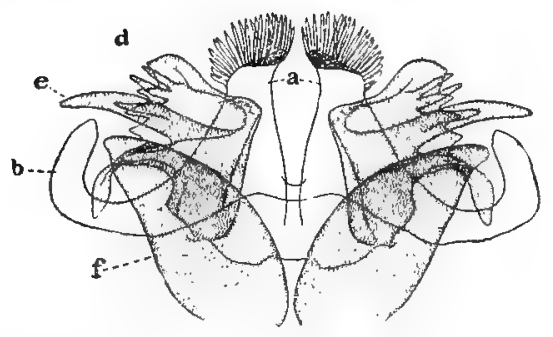

138

MALE GENITALIA.

I28. Culex quinquefasciatus Say.

r29. The same, basal parts enlarged.

I30. Culex quinquefasciatus dipseticus Dyar $\mathcal{E}$ Knab, basal parts enlarged.

131. Culex comitatus Dyar \& Knab, basal parts enlarged.

I32. Culex proximus Dyar \& Knab.

I33. The same, basal parts enlarged.

I34. Culex sphinx Hozvard, Dyar \& Knab. 


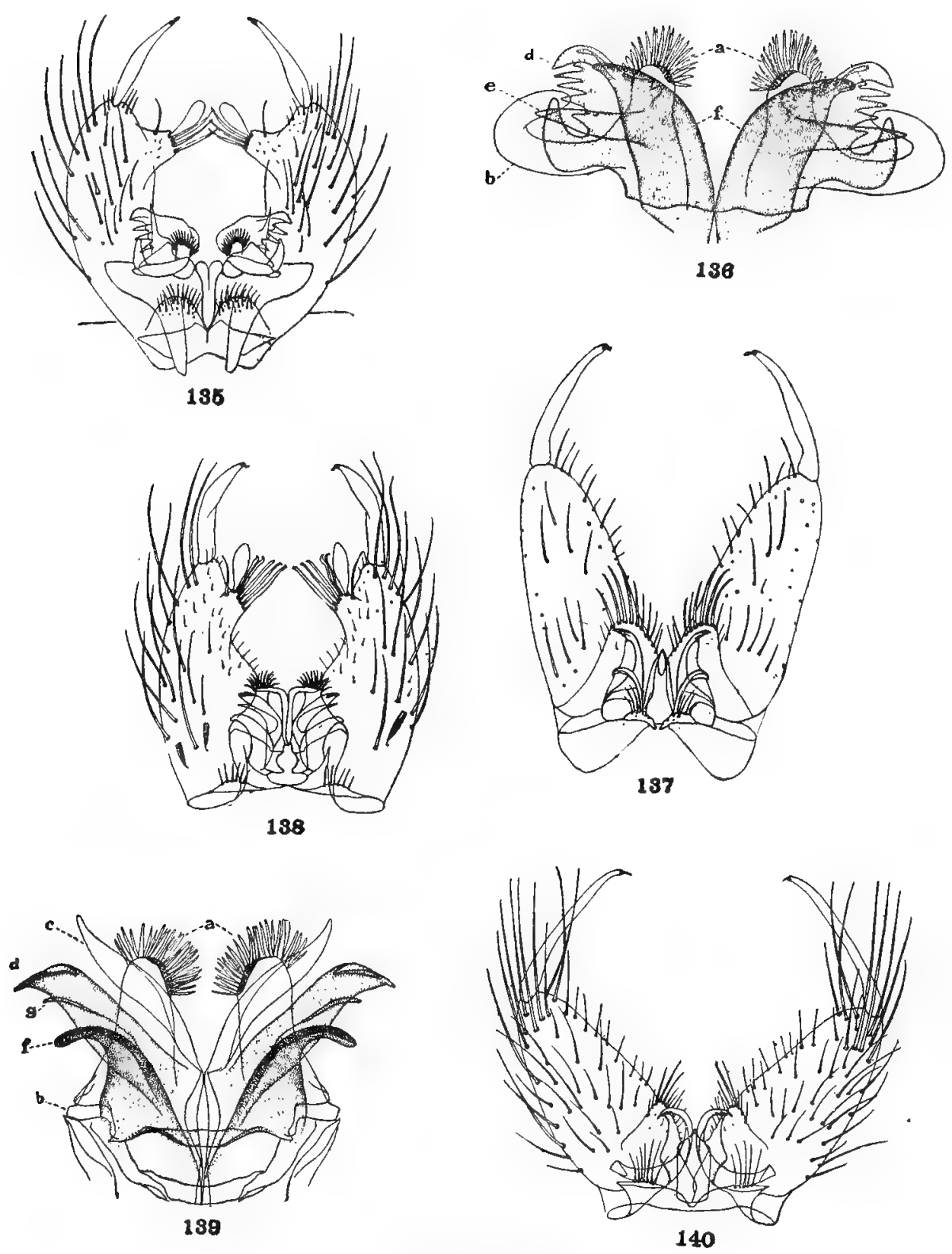

MALE GENITALIA.

I35. Culex similis Theobald.

I36. The same, basal parts enlarged.

I37. Culex dyari Coquillett.

138. Culex pipiens Linnaus.

139. The same, basal parts enlarged.

I40. Culex melanurus Coquillett. 


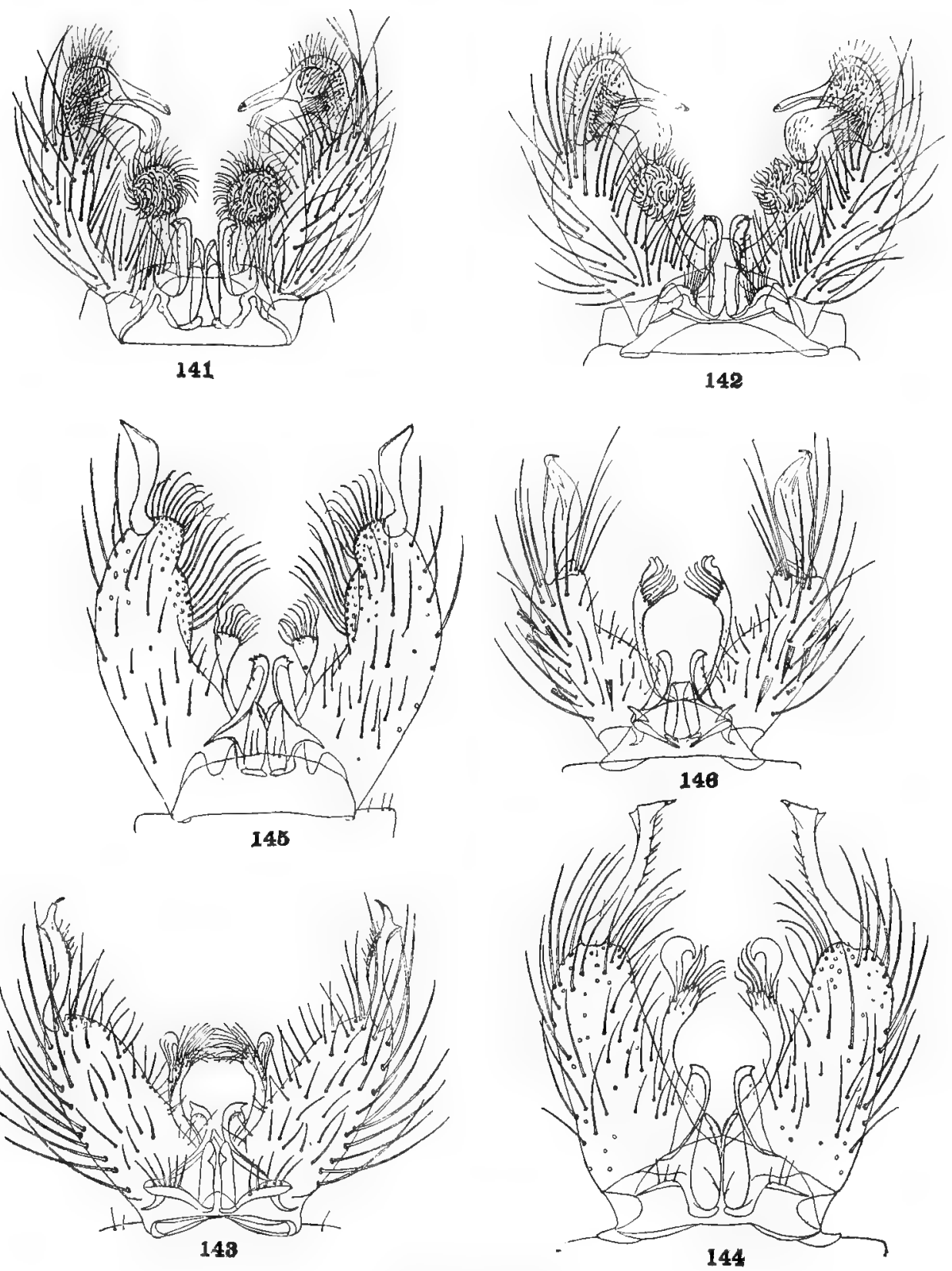

MALE GENITALIA.

I4I. Psorophora virescens Dyar \& $K n a b$.

I42. Psorophora howardii Coquillett.

I43. Psorophora ciliata Fabricius.

I44. Psorophora cilipes Fabricius.

I45. Psorophora sæva Dyar \& $K$ Knab.

I46. Psorophora sayi Dyar \& Knab. 


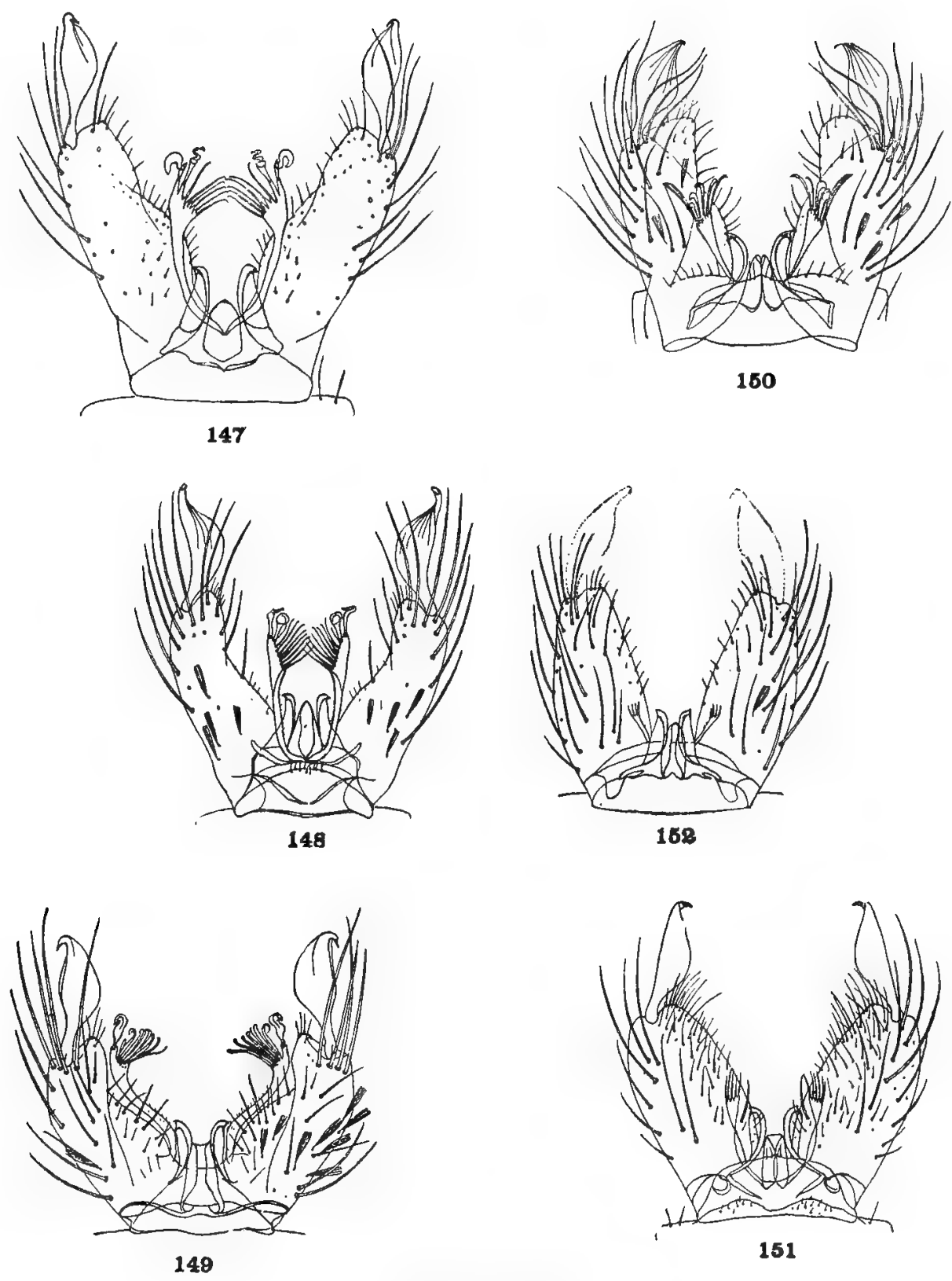

MALE GENITALIA.

I47. Psorophora horridus Dyar \& $K n a b$.

148. Psorophora pazosi Pazos.

I49. Psorophora posticatus Wiedemann.

I50. Psorophora discolor Coquillett.

I5I. Psorophora pygmæa Theobald.

152. Psorophora insularius Dyar \& Knab. 


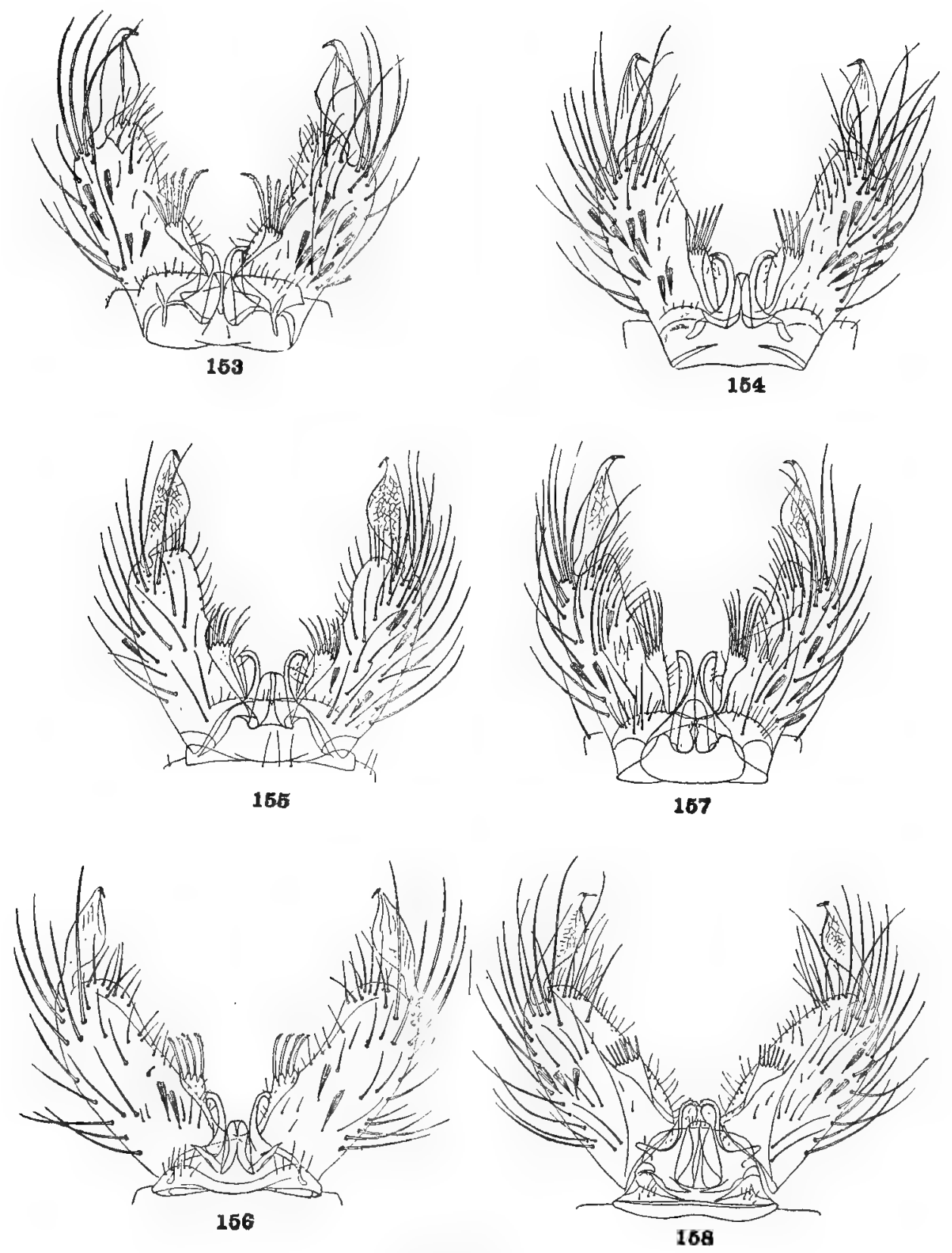

MALE GENITALIA.

I53. Psorophora floridense Dyar \& Knab.

154. Psorophora jamaicensis Theobald.

I55. Psorophora toltecum Dyar \& Knab.

I56. Psorophora columbia Dyar \& Knab.

I57. Psorophora cingulatus Fabricius.

I58. Psorophora cyanescens Coquillett. 


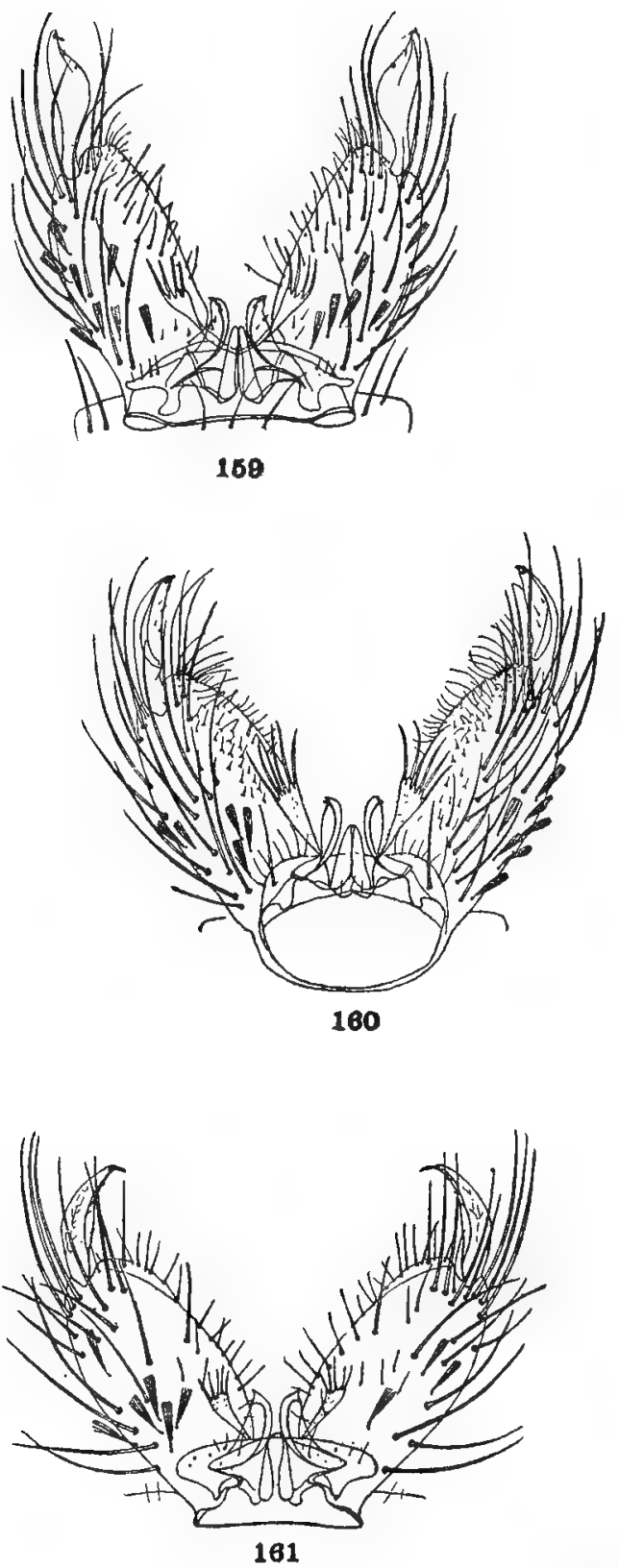
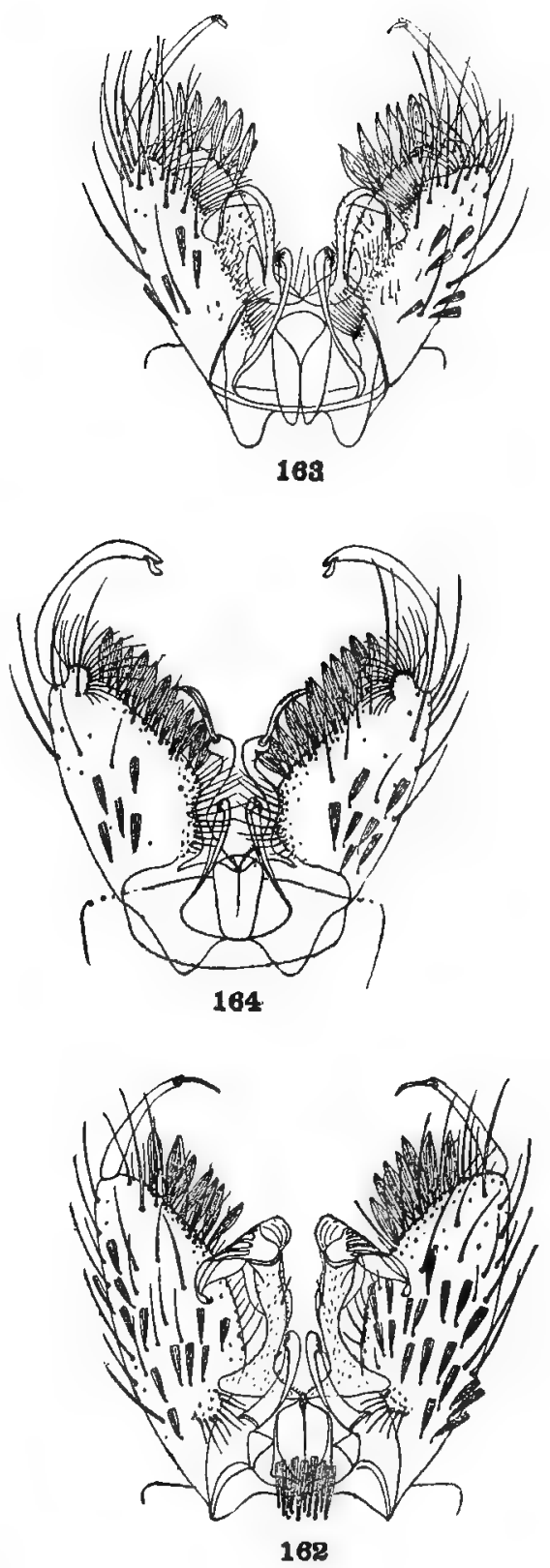

MALE GENITALIA.

159. Psorophora haruspicus Dyar \& Knab.

160. Psorophora infine Dyar \& Knab.

I6r. Psorophora signipennis Coquillett.

I62. Stegoconops equinus Theobald.

163. Stegoconops albomaculatus Theobald.

164. Stegoconops lucifer Howard, Dyar \& Knab. 


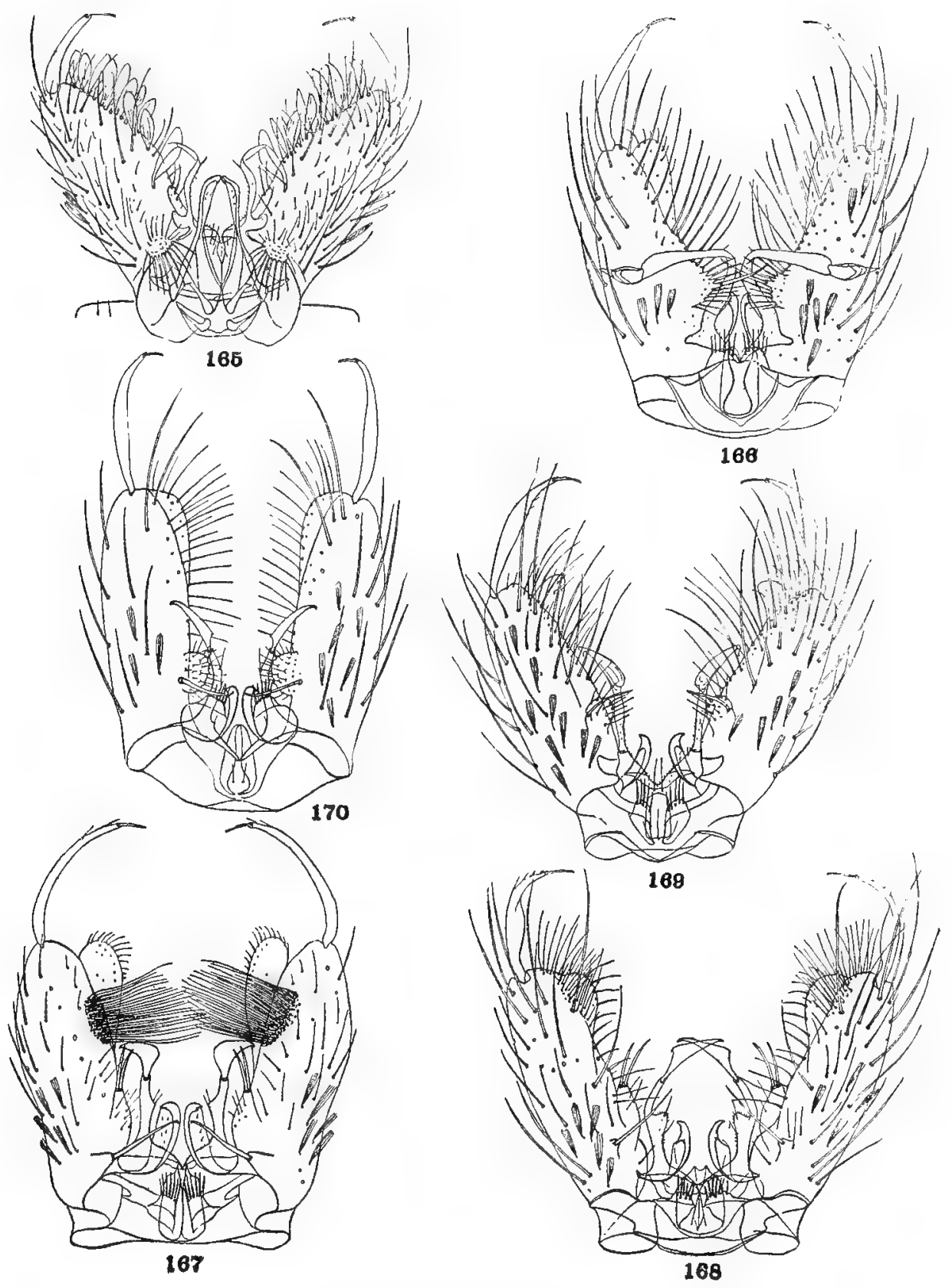

MALE GENITALIA.

165. Stegoconops capricornii Lutz.

I66. Aëdes trichurus Dyar.

I67. Aëdes diantæus Howard, Dyar \& Knab.

168. Aëdes impiger Walker.

169. Aëdes pertinax Grabham.

I7o. Aëdes bimaculatus Coquillett. 


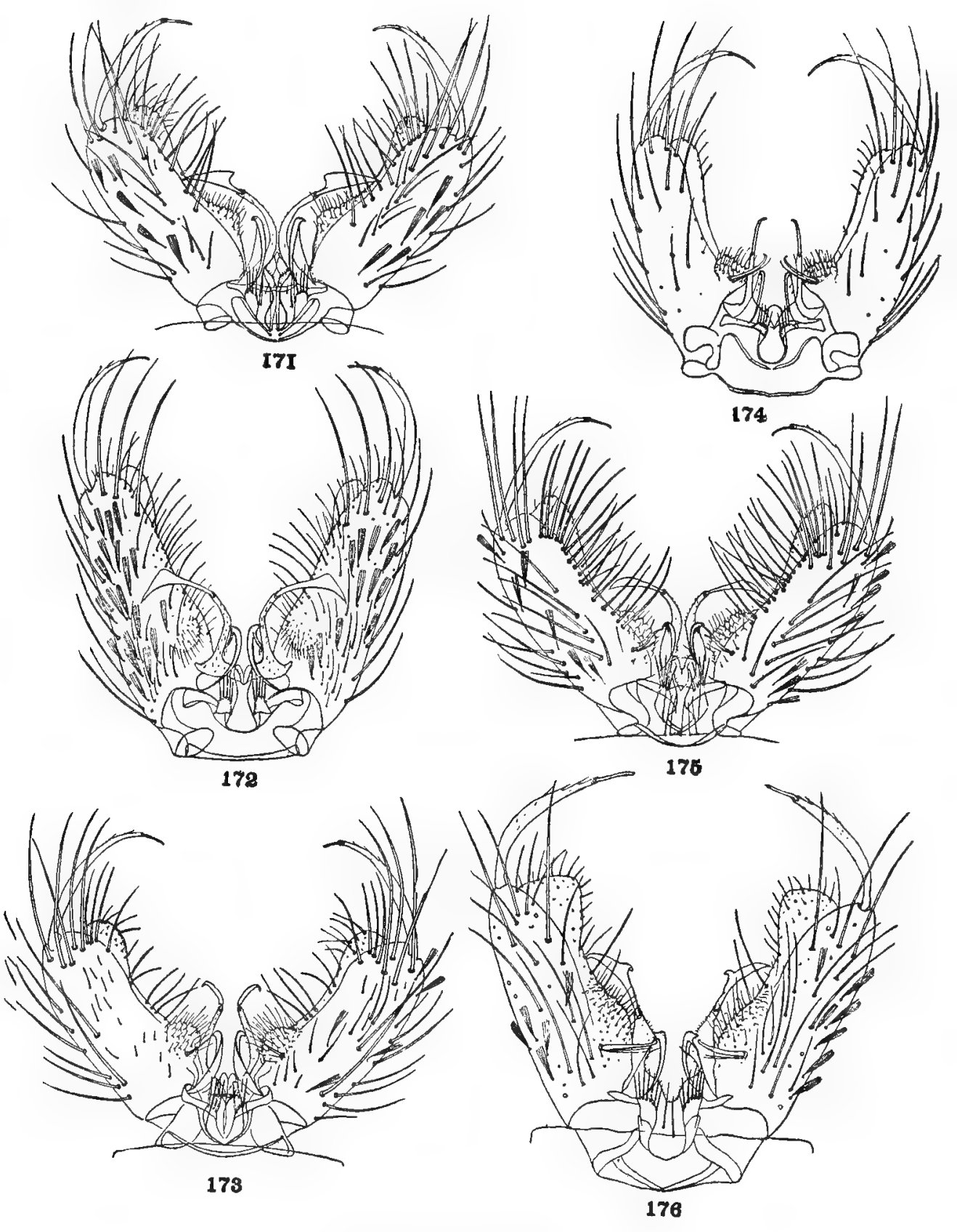

MALE GENITALIA,

I7I. Aëdes abfitchii Felt.

172. Aëdes abfitchii Felt.

173. Aëdes fitchii Felt \& Young.

174. Aëdes curriei Coquillett.

175. Aëdes stimulans Walker.

176. Aëdes fletcheri Coquillett. 


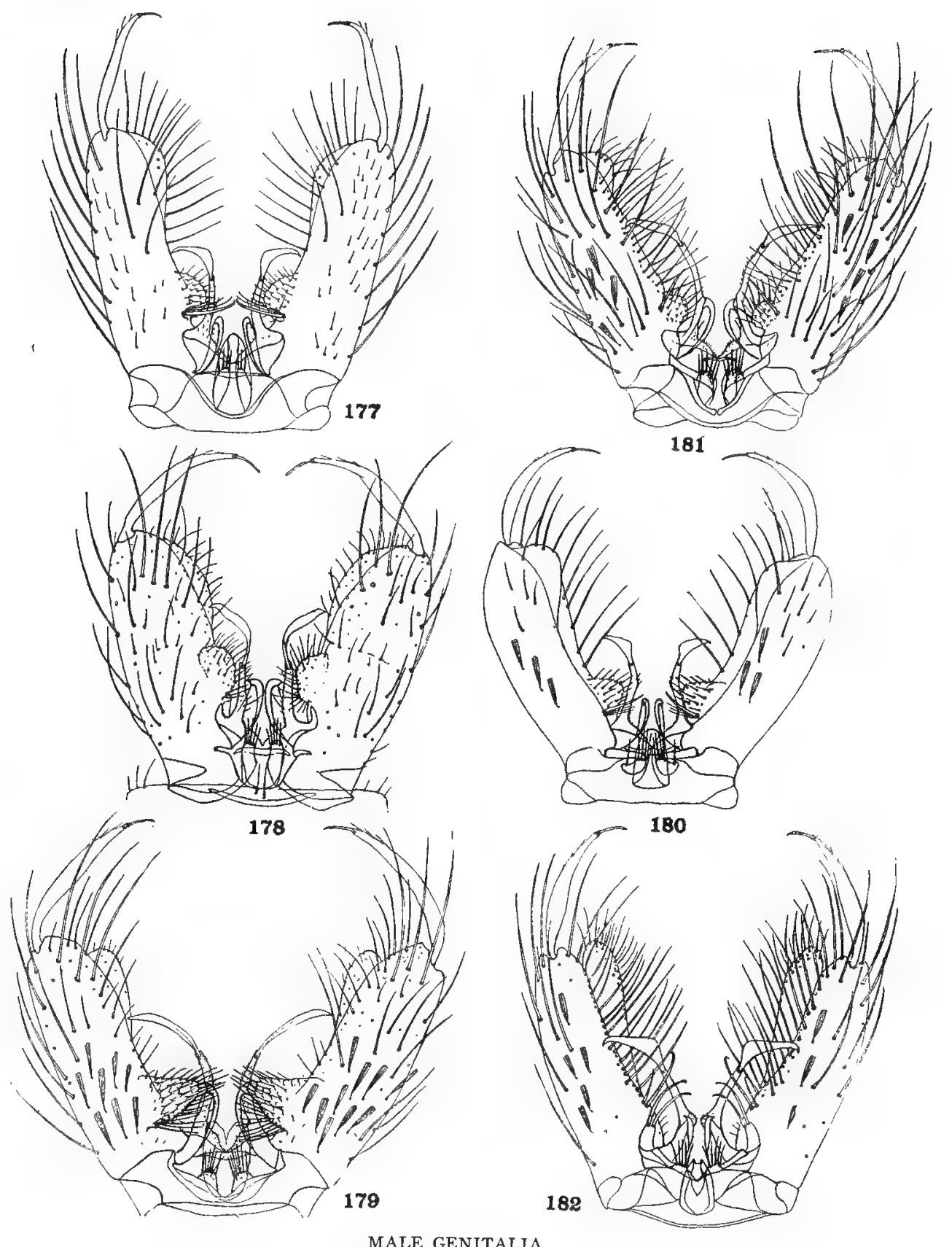

MALE GENITALIA.

I77. Aëdes auroides Felt.

I78. Aëdes campestris Dyar \& $K n a b$.

179. Aëdes riparius Dyar \& $K n a b$.

I80. Aëdes hirsuteron Theobald.

18r. Aëdes lazarensis Felt \& Y Young.

I82. Aëdes pullatus Coquillett. 


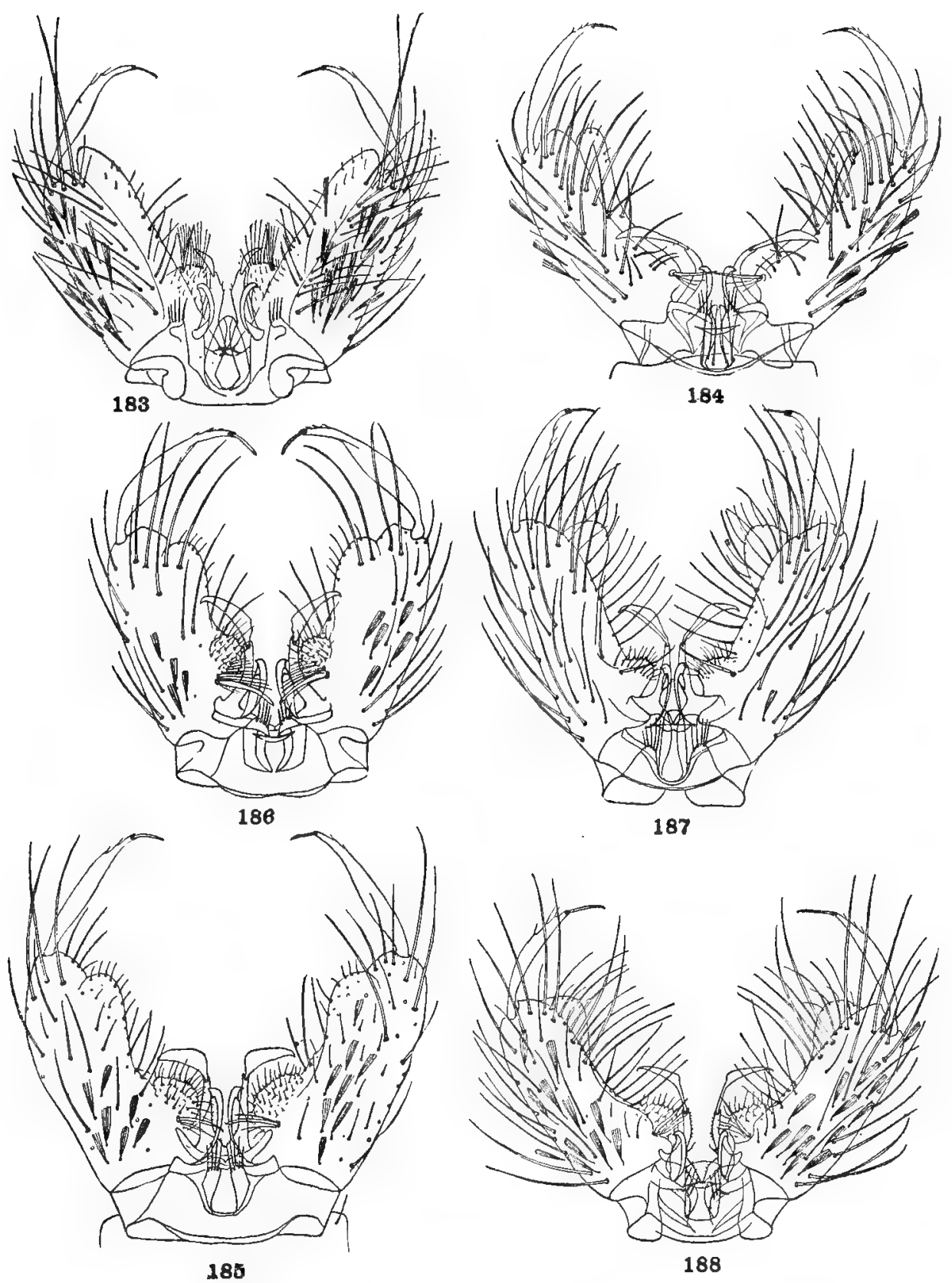

187

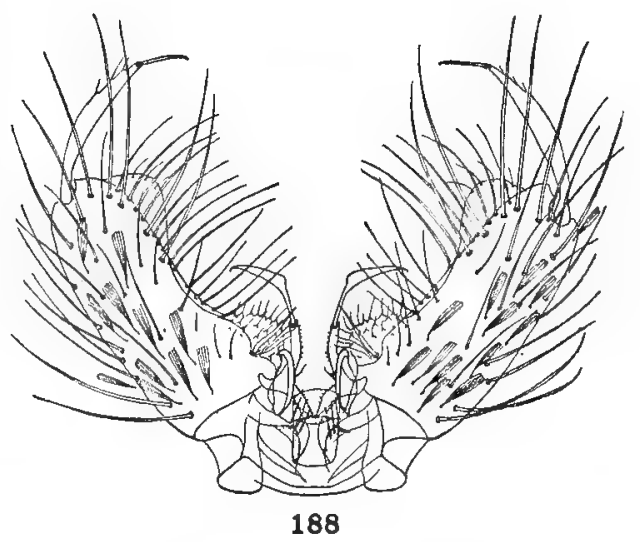

MALE GENITALIA.

183. Aëdes æestivalis Dyar.

184. Aëdes cantator Coquillett.

I85. Aëdes spencerii Theobald.

186. Aëdes idahoensis Theobald.

I8. Aëdes grossbecki Dyar \& Knab.

I88. Aëdes squamiger Coquillett. 


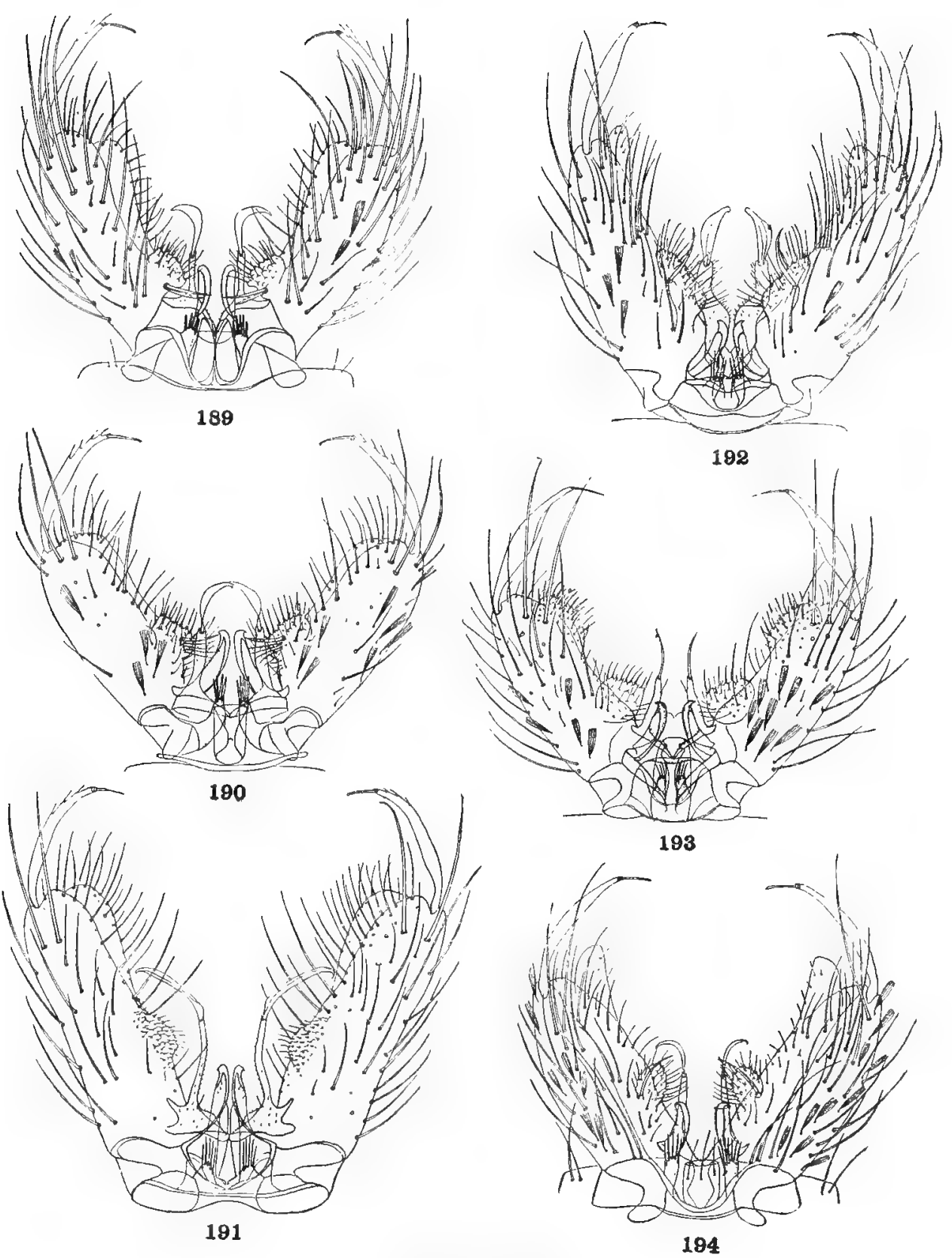

MALE GENITALIA.

I89. Aëdes curriei Coquillett.

I90. Aëdes onondagensis quaylei Dyar \& Knab.

I9I. Aëdes euedes Howard, Dyar \& $K n a b$.

192. Aëdes atlanticus Dyar \& Knab.

I93. Aëdes canadensis Theobald.

194. Aëdes serratus Theobald. 


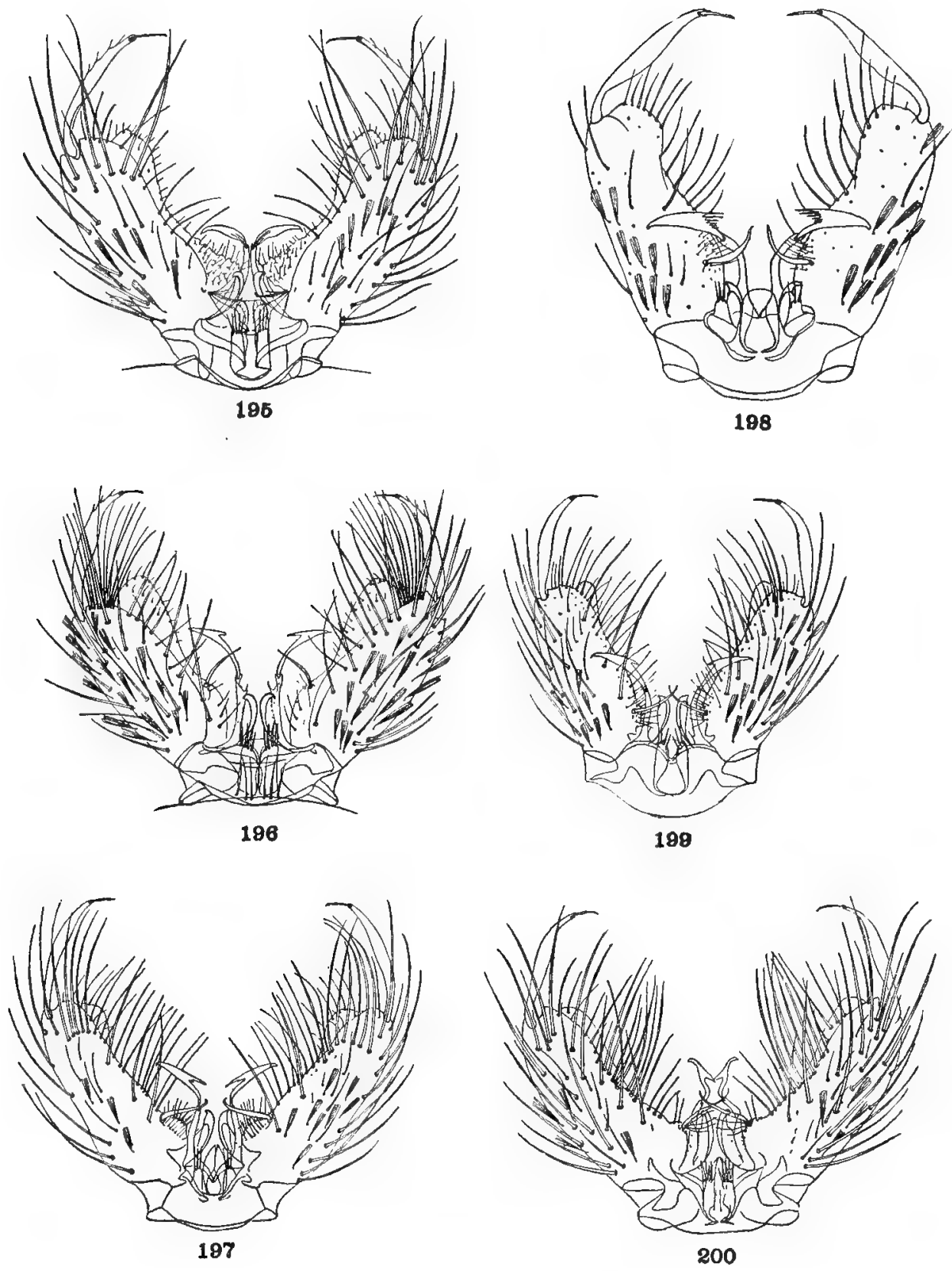

MALE GENITALIA.

195. Aëdes abserratus Felt \& Young.

196. Aëdes aurifer Coquillett.

197. Aëdes tortilis Theobald.

198. Aëdes cuneatus Dyar \& $K n a b$

I99. Aëdes scapularis Rondani.

200. Aëdes infirmatus $D_{\text {yar }} \mathcal{\&} K n a b$. 


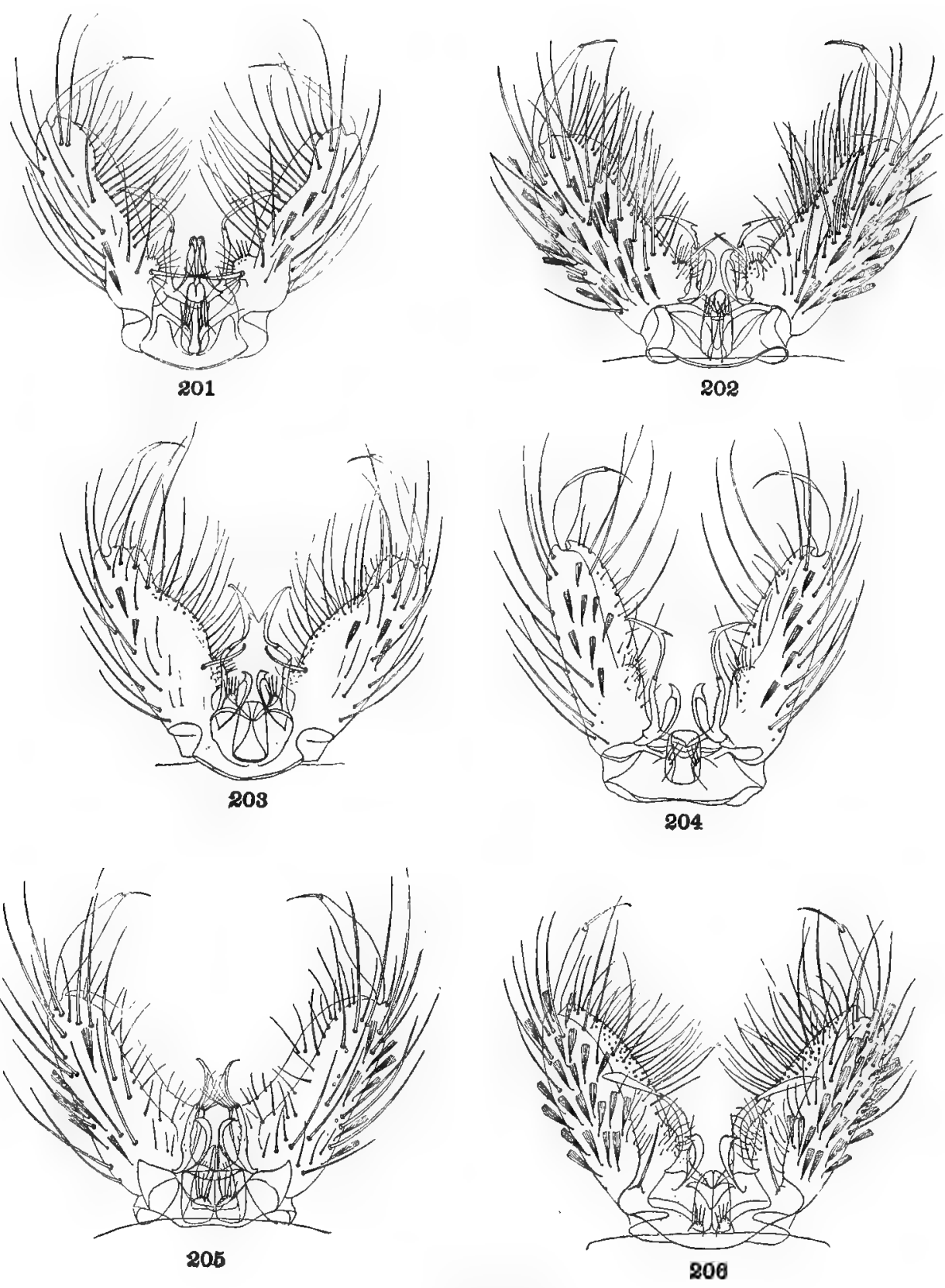

MALE GENITALIA.

20I. Aëdes trivittatus Coquillett.

202. Aëdes scapularis Rondani.

203. Aëdes plutocraticus Dyar \& Knab.

204. Aëdes niger Giles.

205. Aëdes tæniorhynchus Wiedemann.

206. Aëdes mediovittata Coquillett. 


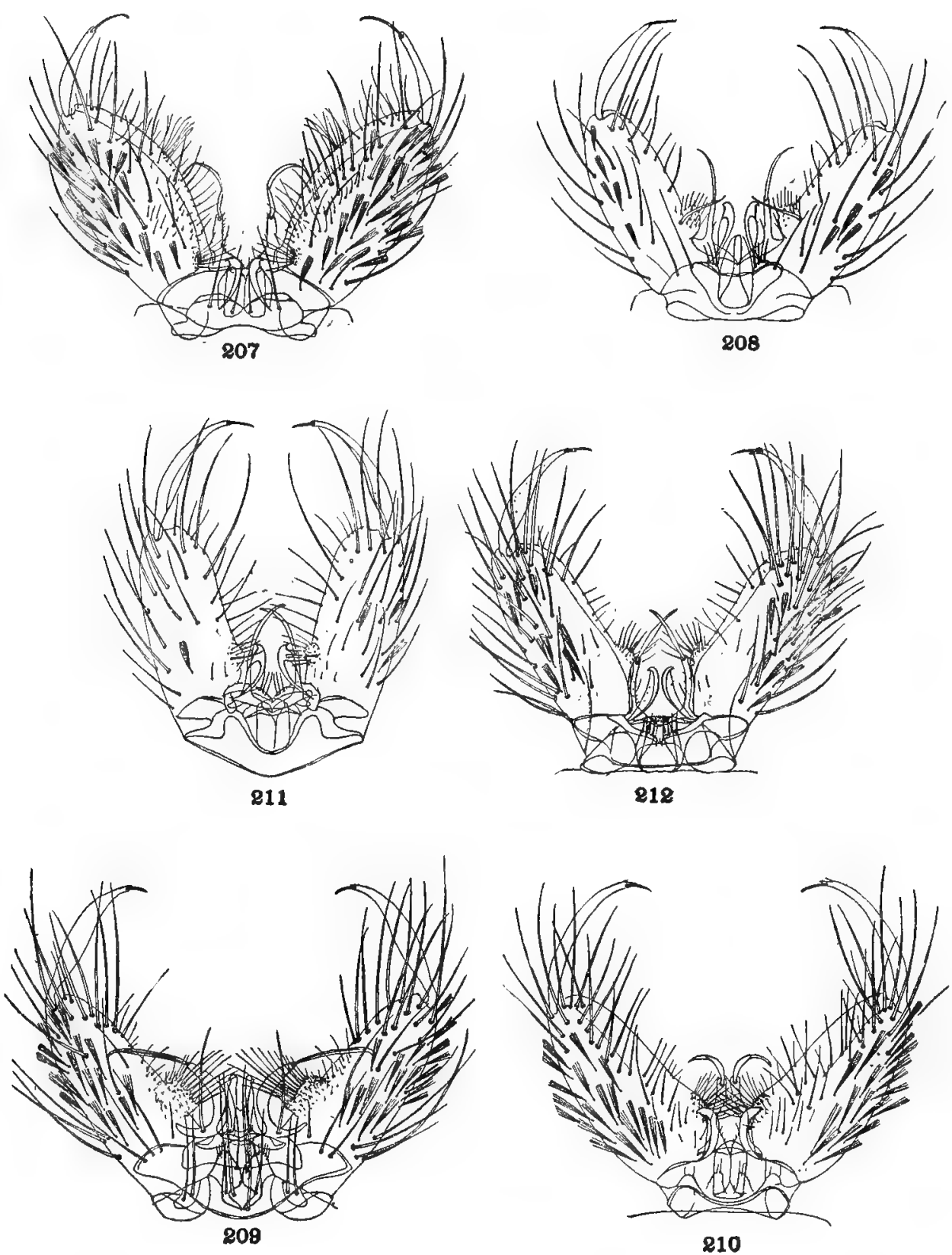

MALE GENITALIA.

207. Aëdes triseriatus Say.

208. Aëdes dupreei Coquillett.

209. Aëdes varipalpus Coquillett.

21o. Aëdes atropalpus Coquillett.

2II. Aëdes sollicitans $W$ alker.

2I2. Aëdes mitchellæ Dyar. 


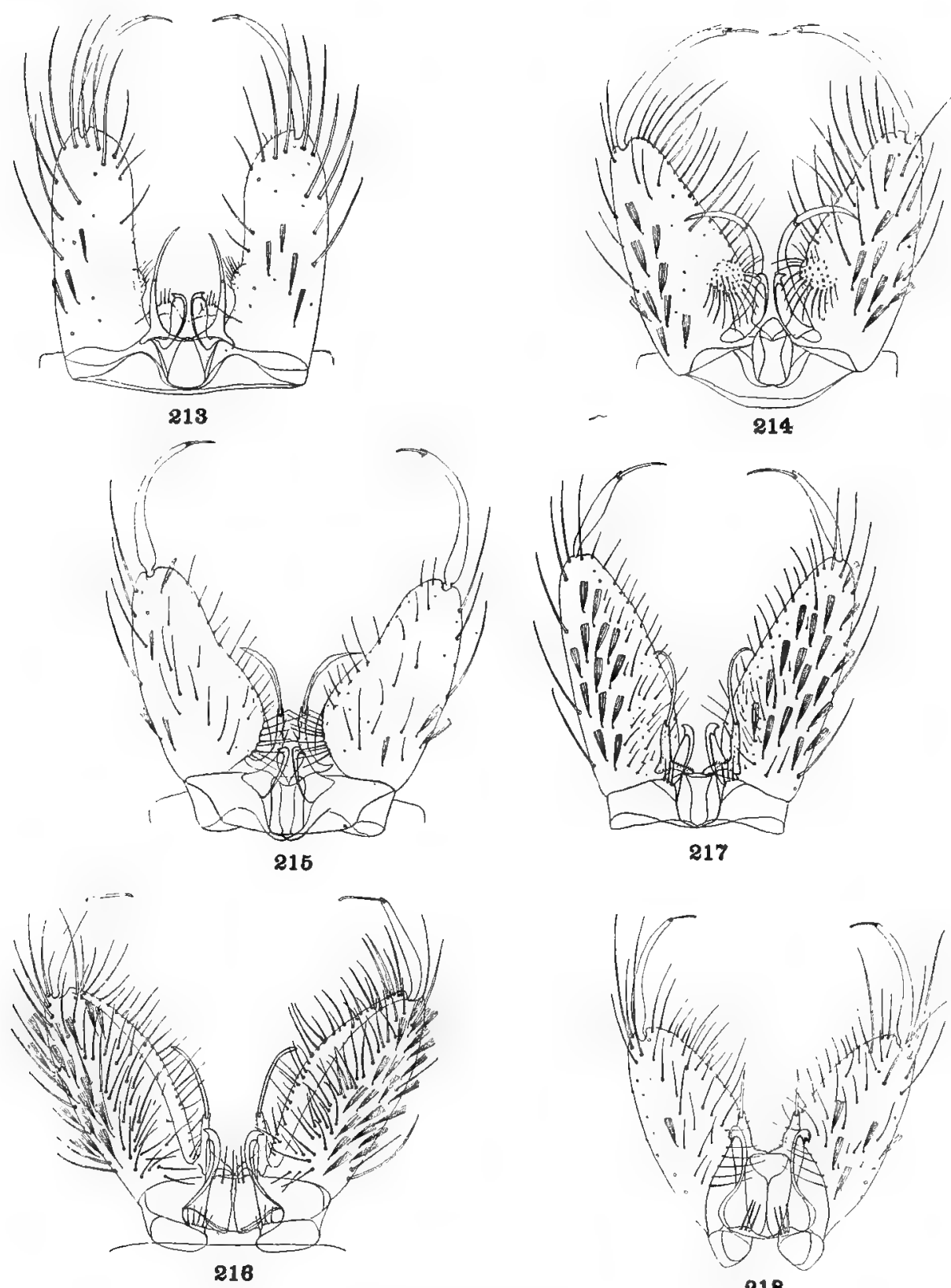

MALE GenitaliA.

2r3. Aëdes nigromaculis Ludlow.

2I4. Aëdes epactius Dyar \& Knab.

2I5. Aëdes fluviatilis Lutz.

216. Aëdes podographicus Dyar \& $K n a b$.

2I7. Aëdes thorntoni Dyar $\mathcal{E} K n a b$.

218. Aëdes aureostriata Grabham. 


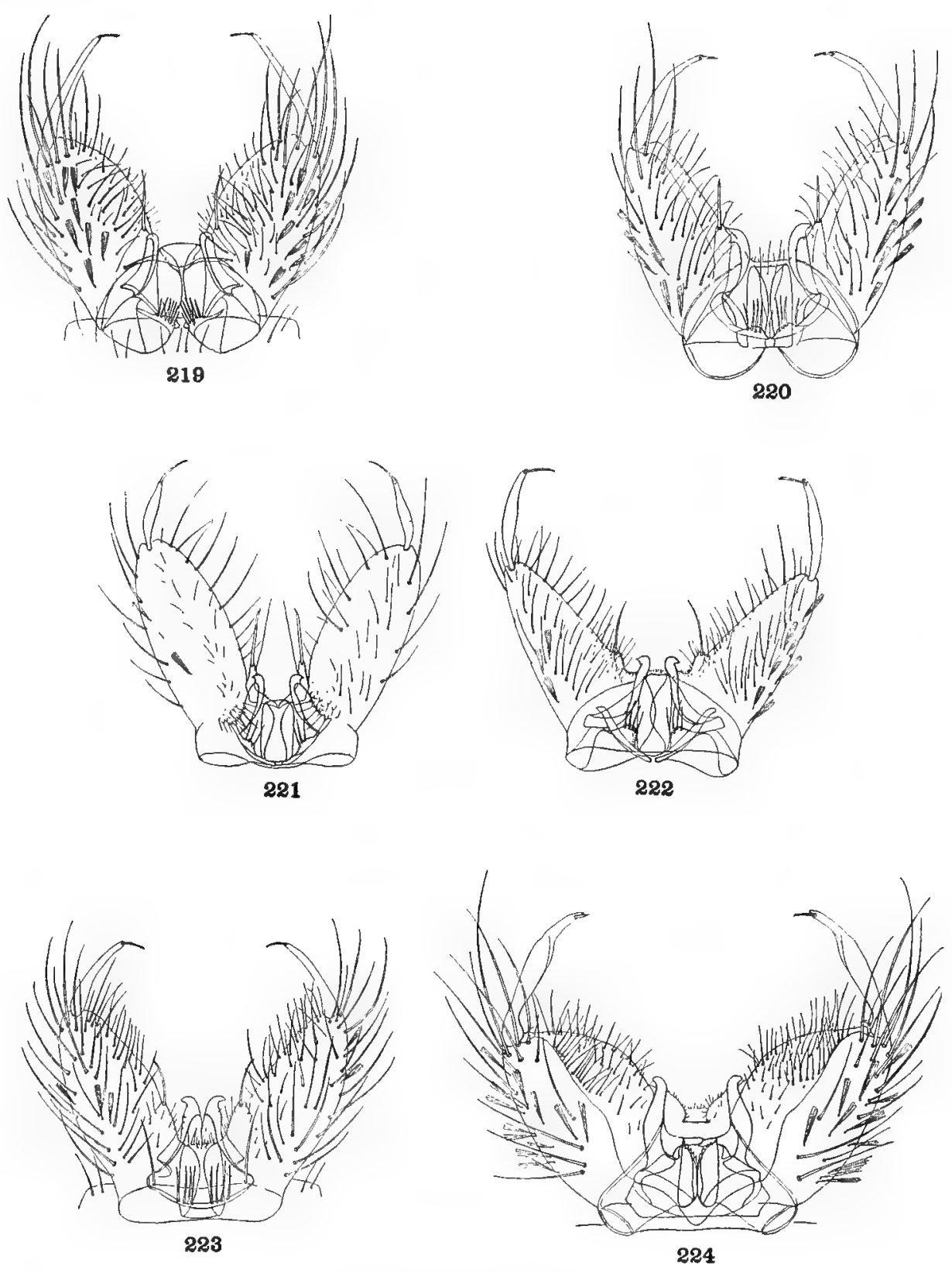

MALE GENITALIA.

2r9. Aëdes albonotata Coquillett.

220. Aëdes busckii Coquillett.

22I. Aëdes oswaldi Lutz.

222. Aëdes fulvithorax Lutz.

223. Aëdes walkeri Theobald.

224. Aëdes calopus Meigen. 


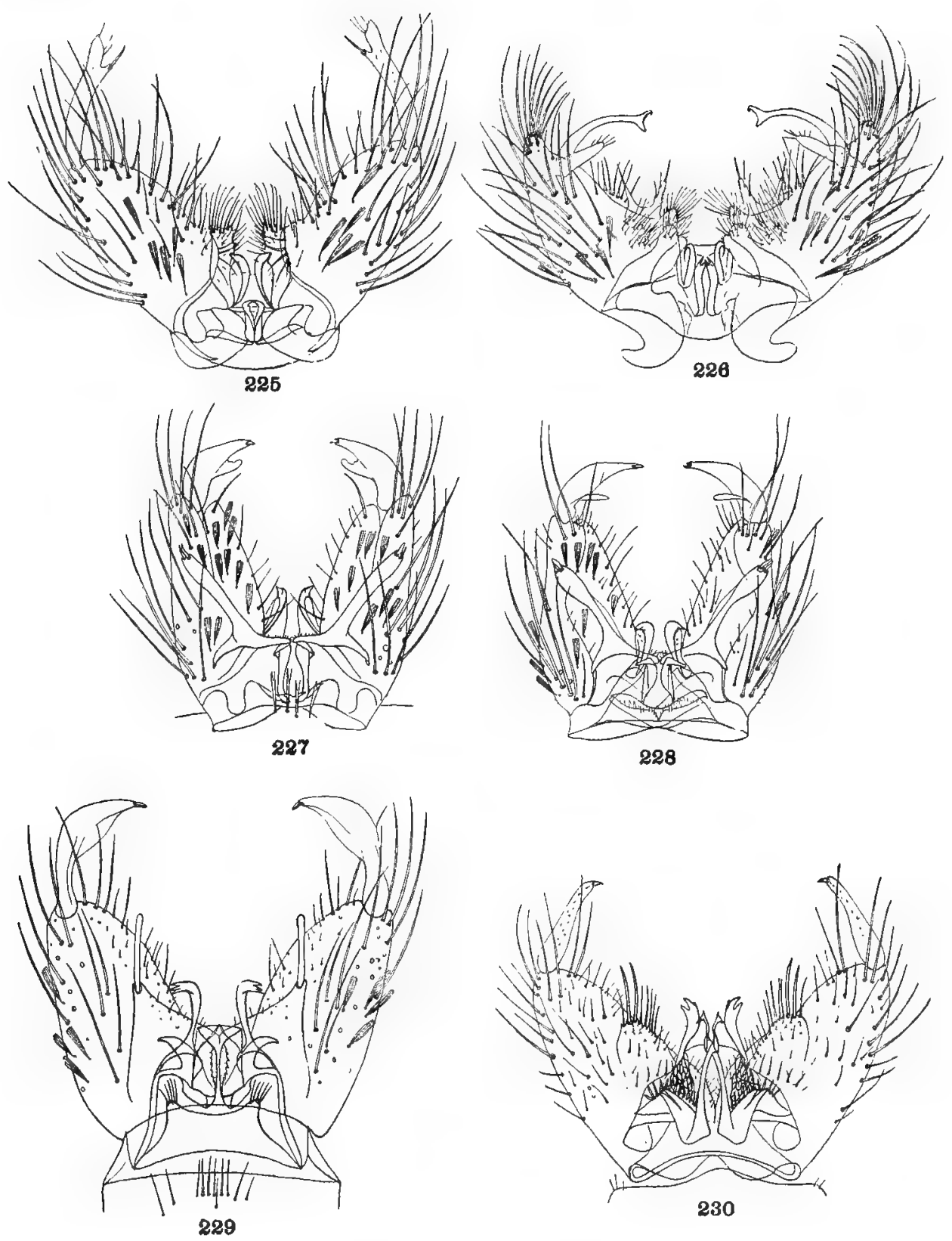

MALE GENITALIA.

225. Aëdes sylvestris Theobald.

226. Aëdes fuscus Osten Sacken.

227. Mansonia flaveolus Coquillett.

228. Mansonia titillans Walker.

229. Mansonia perturbans Walker.

230. Culiseta inornatus Williston. 


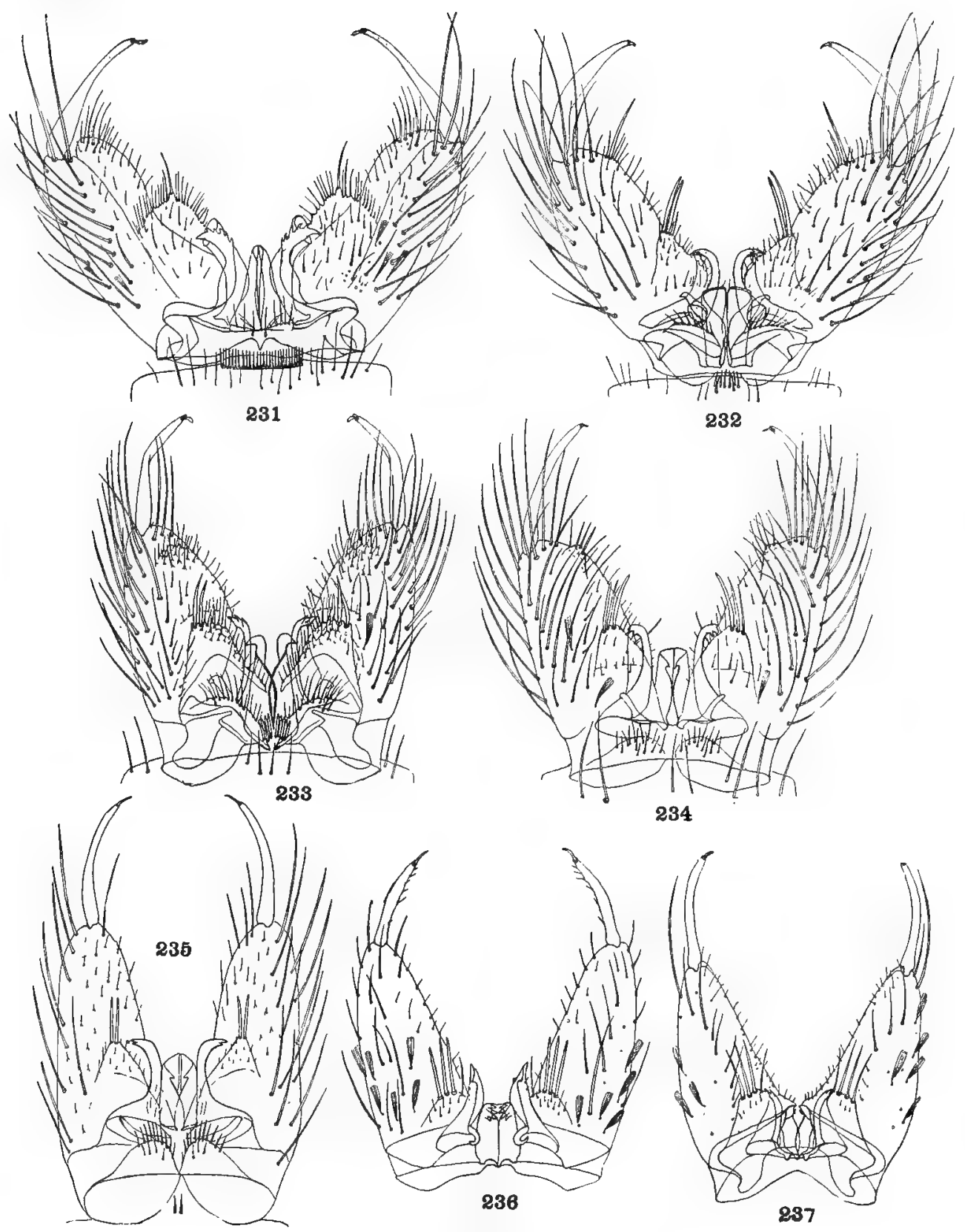

MALE GENITALIA.

231. Culiseta impatiens Walker.

232. Culiseta incidens Thomson.

233. Culiseta alaskaensis Ludlow.

234. Culiseta maccrackenæ Dyar \& Kuab.

235. Culiseta dugesi Dyar \& Knab.

236. Bancroftia phyllozoa Dyar \& Knab.

237. Bancroftia waverleyi Grabham. 


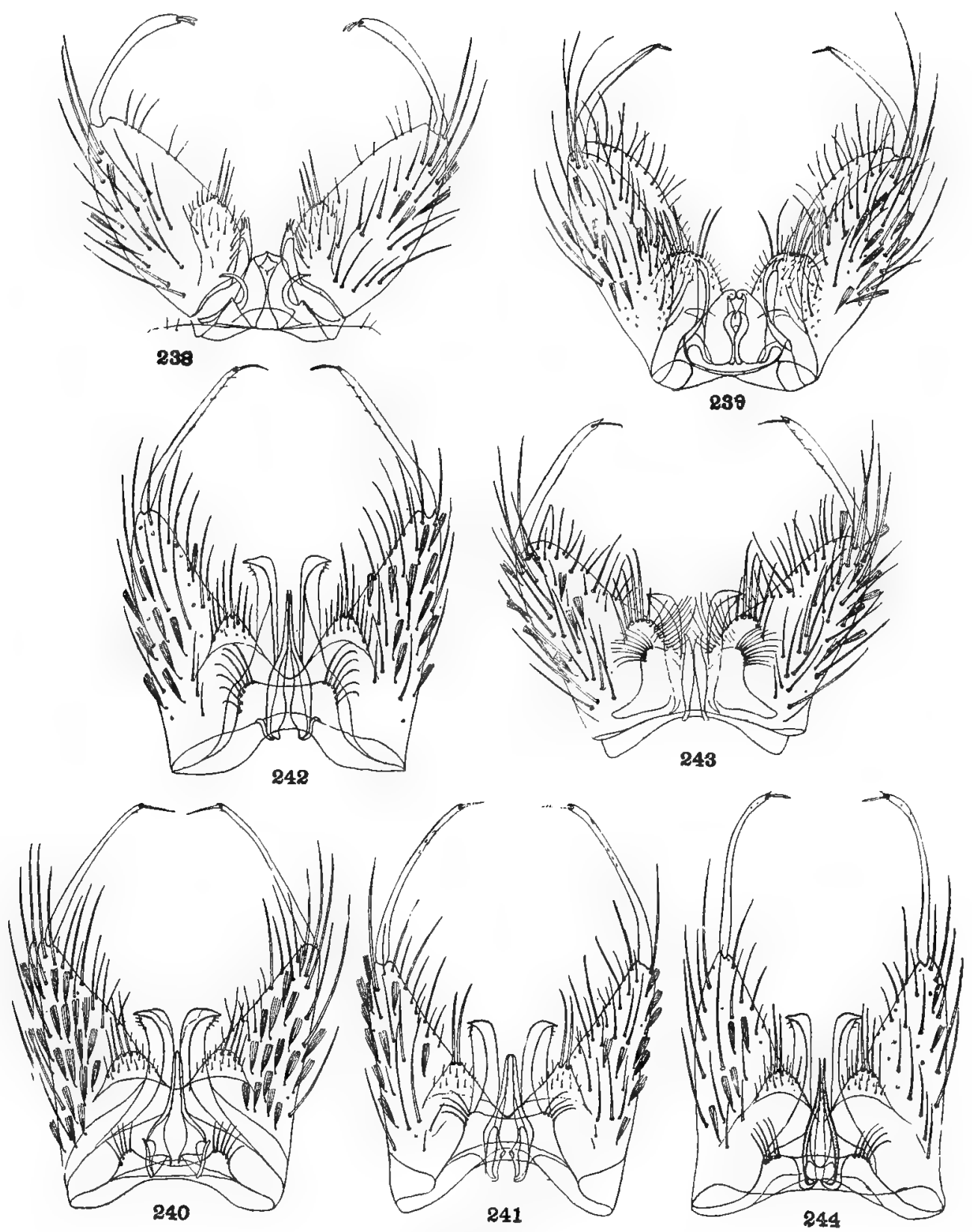

MALE GENITALIA.

238. Bancroftia signifer Coquillett.

239. Bancroftia fascipes Coquillett.

240. Megarhinus portoricensis Von Roeder.

24I. Megarhinus hypoptes $K n a b$.

242. Megarhinus trinidadensis Dyar \& $K n a b$.

243. Megarhinus rutila Coquillett.

244. Megarhinus guadeloupensis Dyar \& Knab. 


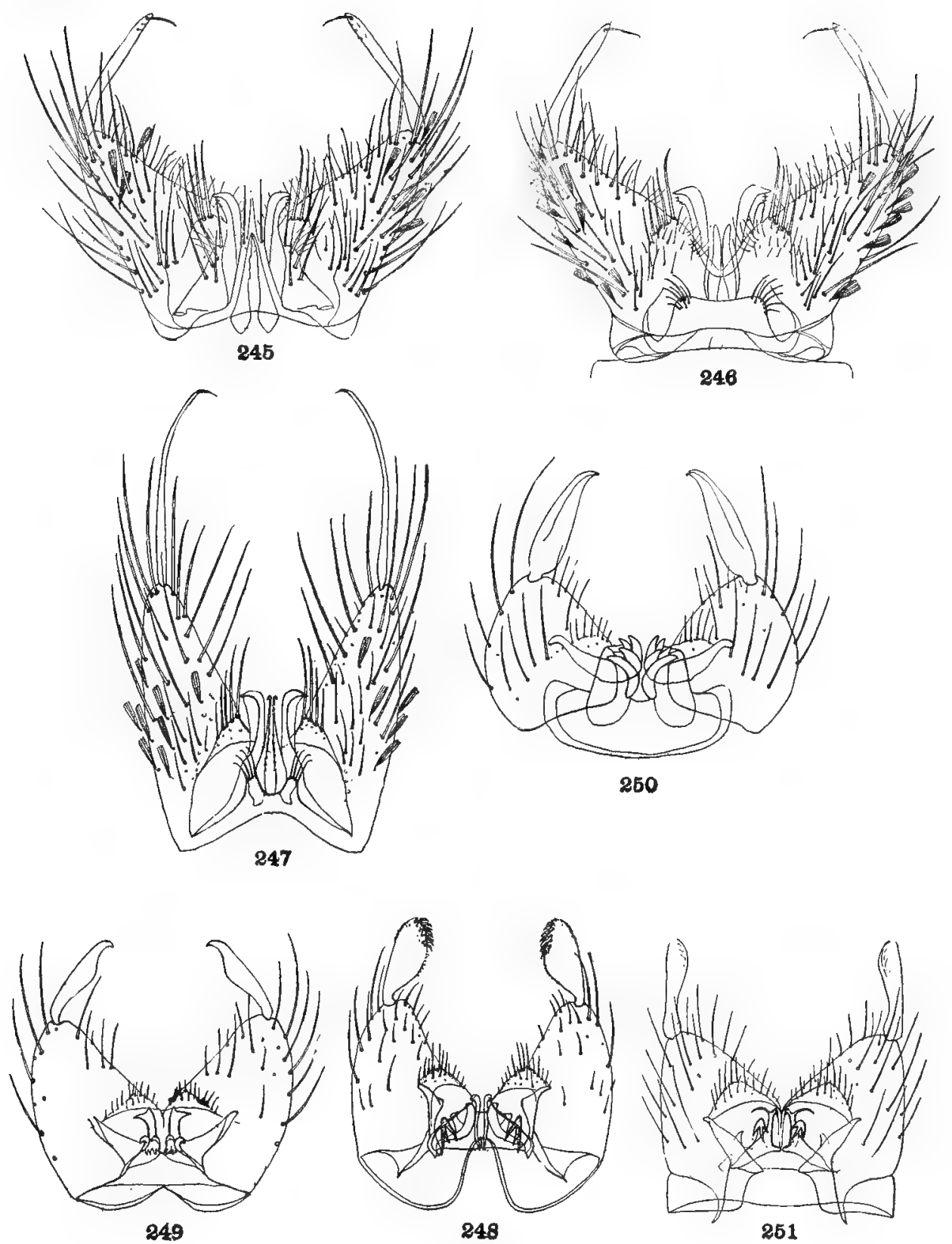

MALE GENITALIA.

245. Megarhinus septentrionalis Dyar \& $K n a b$. 246. Megarhinus moctezuma Dyar \& Knab.

247. Megarhinus superbus Dyar \& Knab.

248. Uranotania lowii Theobald.

249. Uranotrenia socialis Theobald.

250. Uranotænia geometrica Theobald.

25I. Uranotznia basalis Howard, Dyar \& Knab. 


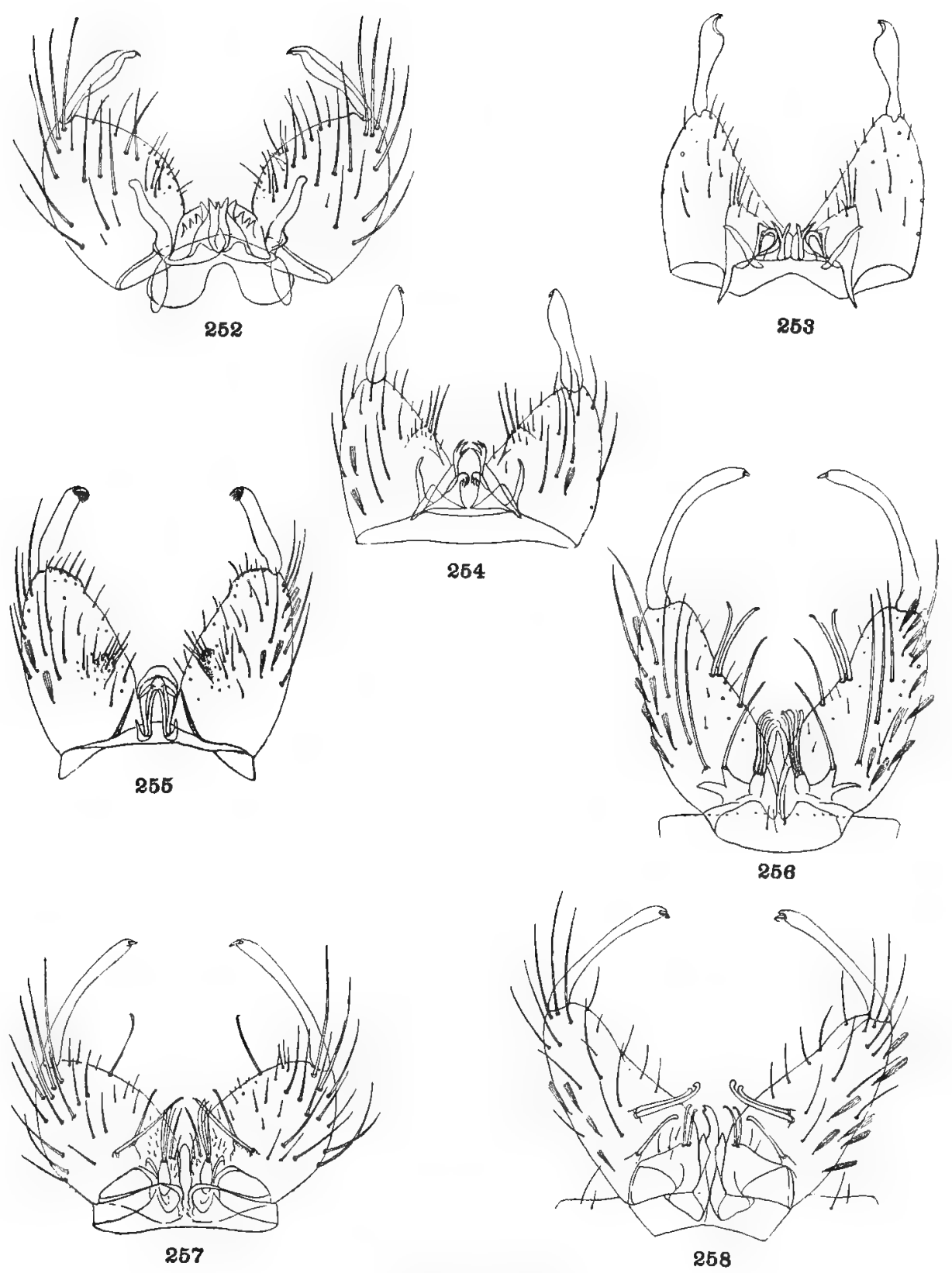

MALE GENITALIA.

252. Uranotænia sapphirinus Osten Sacken.

253. Uranotzenia calosomata Dyar \&o Knab.

254. Uranotænia typhlosomata Dyar \& $K n a b$.

255. Aëdeomyia squamipennis Aribálzaga.

256. Anopheles bellator Dyar $\mathcal{E} K n a b$.

257. Colodiazesis barberi Coquillett.

258. Anopheles argyritarsis Robineau-Desvoidy. 


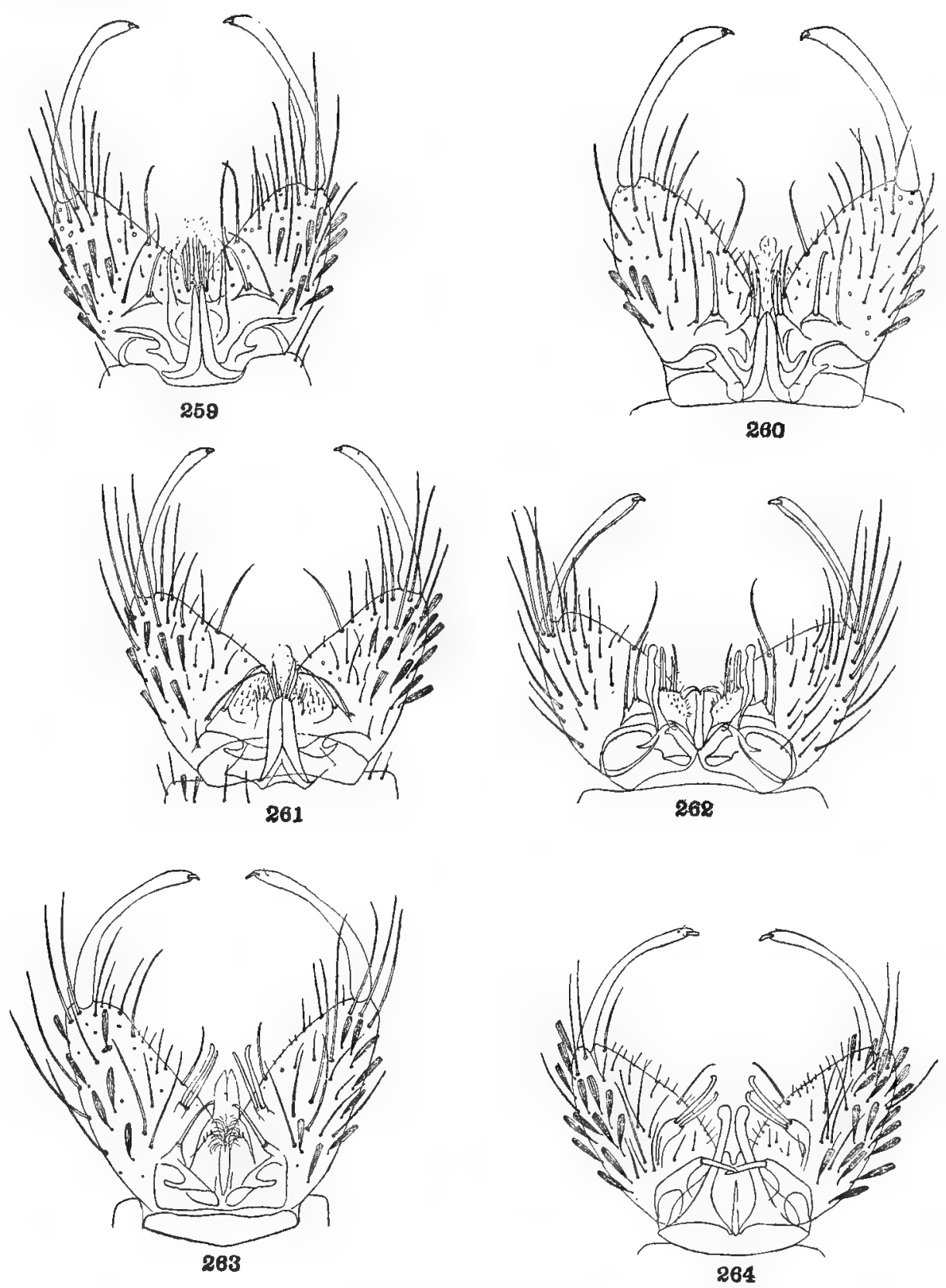

MALE GENITALIA.

259. Anopheles malefactor Dyar \& $K n a b$.

260. Anopheles strigimacula Dyar \& $K n a b$.

261. Anopheles apicimacula Dyar \& $K n a b$.

262. Anopheles quadrimaculatus Say.

263. Anopheles tarsimaculata Goeldi.

264. Anopheles albimanus Wiedemann. 


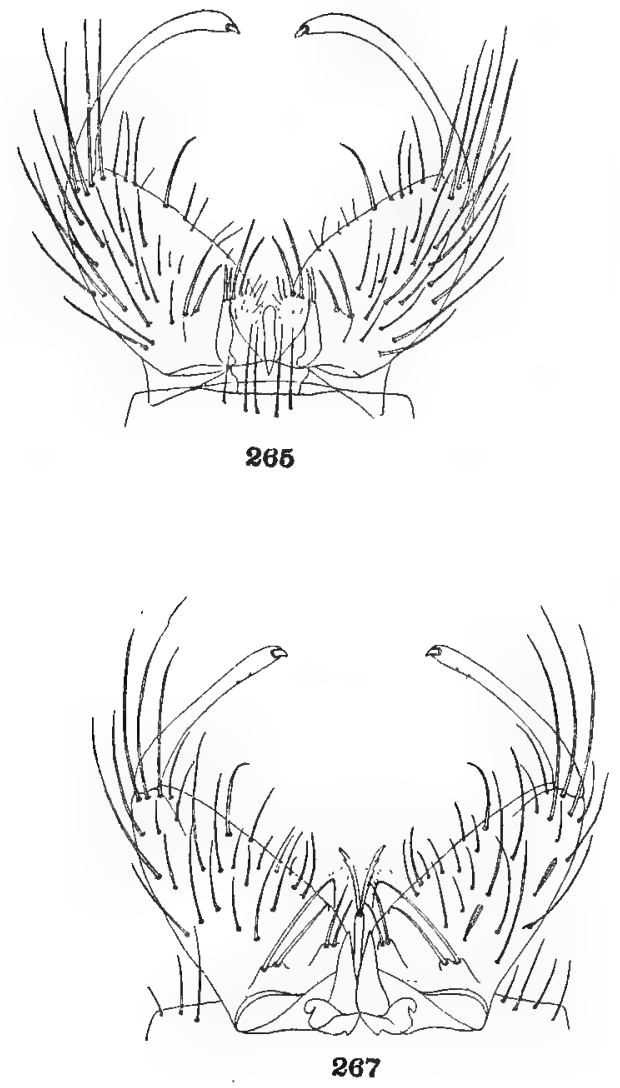
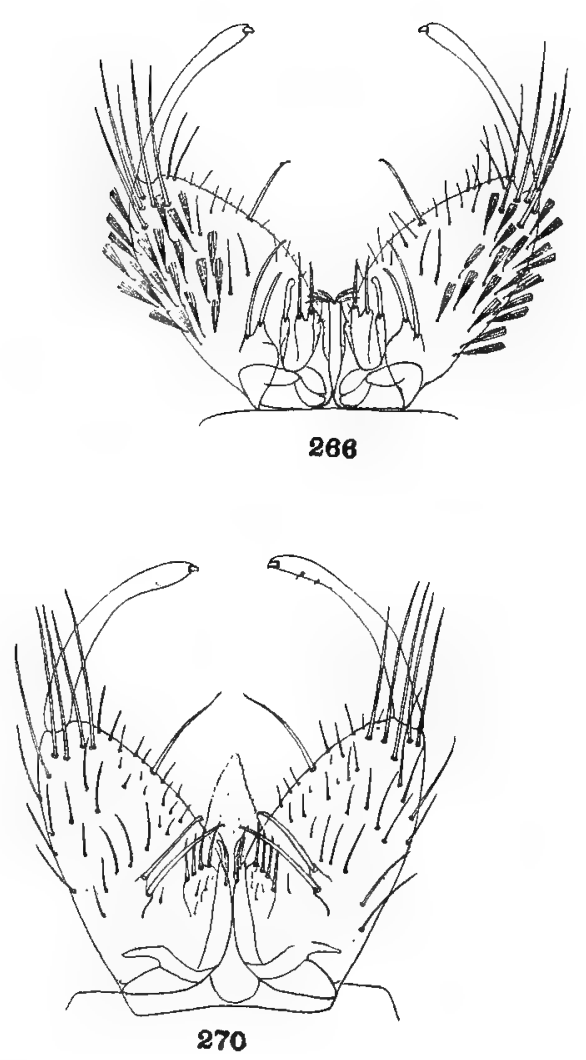

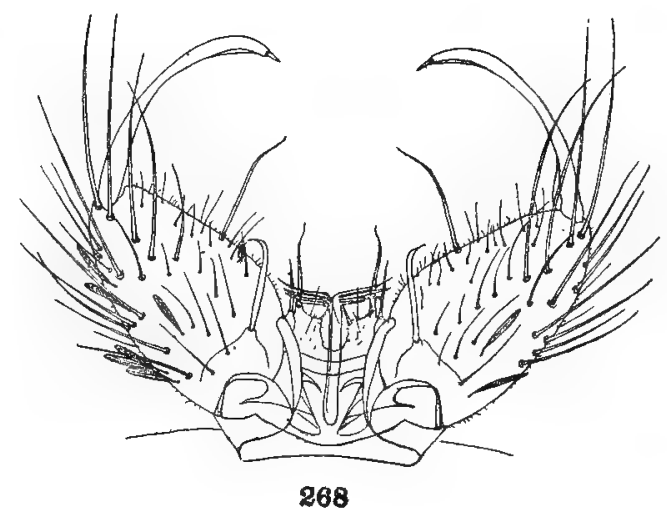

268

MALE GENITALIA.

265. Anopheles pseudopunctipennis Theobald.

266. Anopheles grabhamii Theobald.

26\%. Anopheles eiseni Coquillett.

268. Anopheles punctipennis Say.

269. Anopheles crucians Wiedemann.

270. Anopheles occidentalis Dyar \& Knab. 


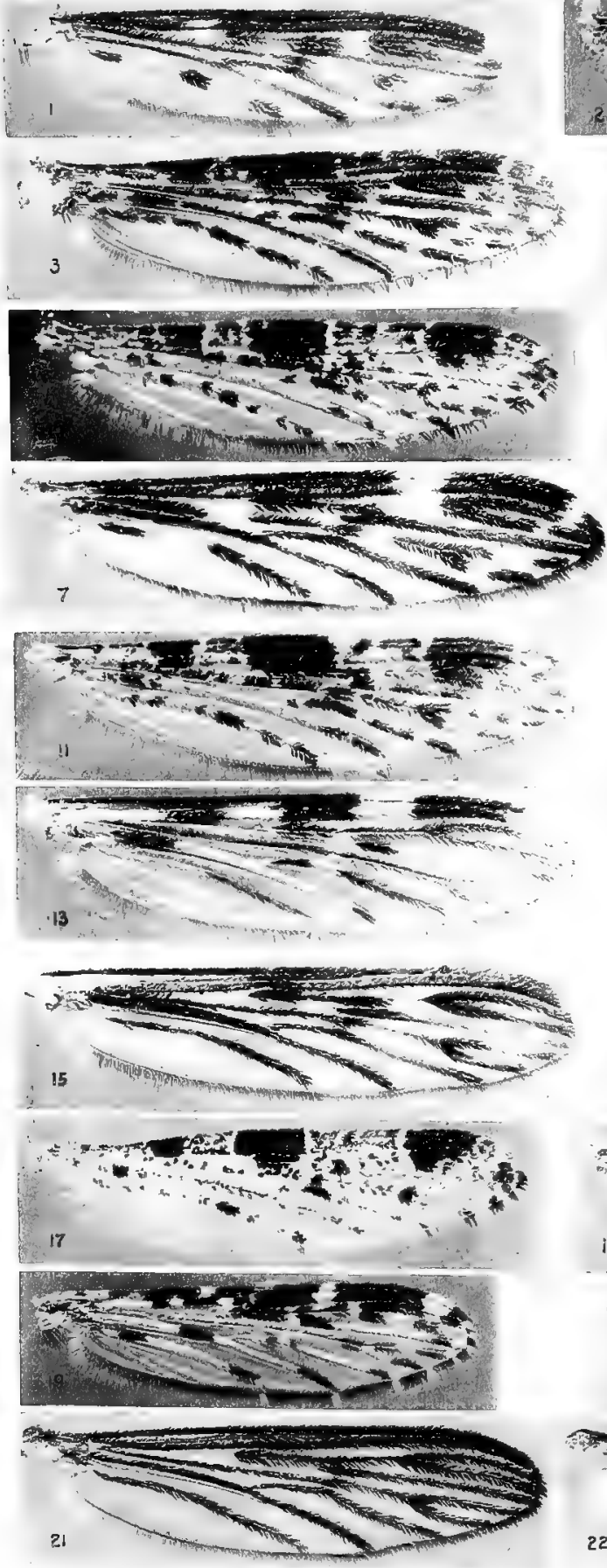
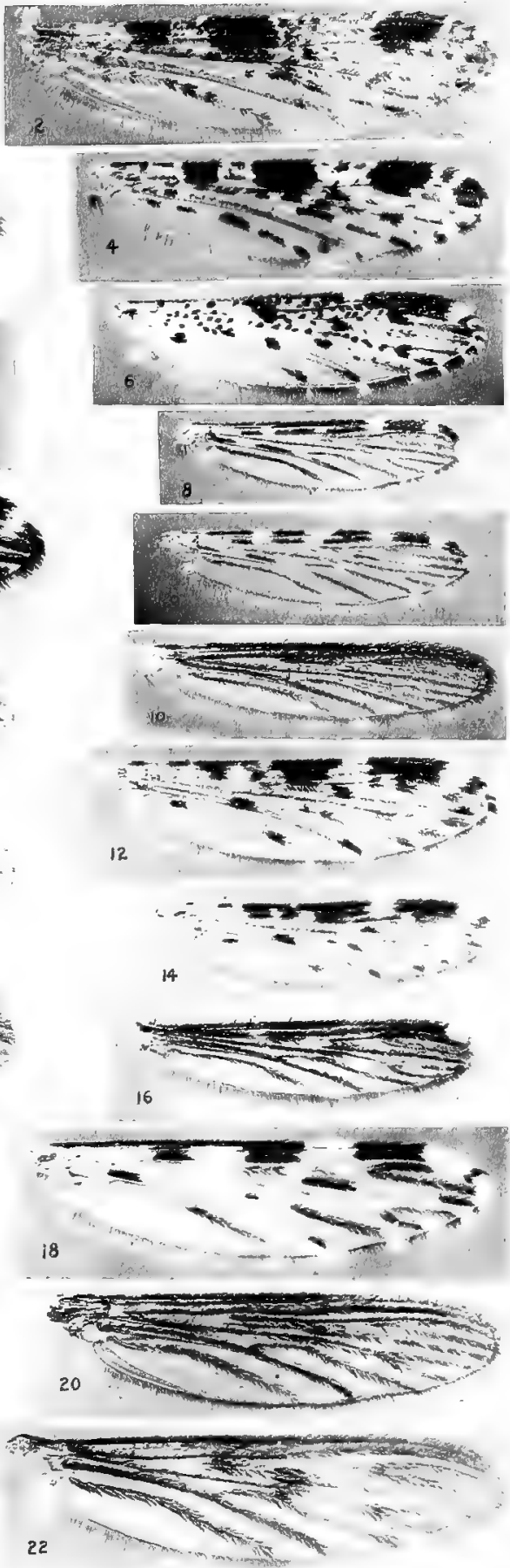

Wings of Different Species of Anopinelines Shown to tite Same Scale.

\section{Anopheles crucians Wied.}

2. A. malefactor $D$. \& $K$.

3. A. vestitipennis $D$. \& $K$.

4. $\Lambda$. apicinacula $D$. \& $K$.

5. A. punctimacula $D$. \& $K$.

6. A, grabhamii Theob.

7. A. punetipennis Say.

8. A. neivai $H ., D . \& K$.
9. A. bellator $D$. $\&$. $K$.

10. Colodiazesis barberi Coq.

11. A. strigimacula $D$. \& $\bar{K}$.

12. A. tarsimaculata Gocldi.

13. A. franciscants $M C C$.

14. A. albimanus Wied.

15. A. occidentalis $D$. \& $K$.
16. $\Lambda$. eiseni Coq.

17. A. mediopunctata Theob.

18. A. pscudopunctipennis Theab.

19. A. argyritarsis Rob.-Des?'.

20. A. atropos $D . \& K$.

21. A. Walkeri Theob.

22. A. quadrimaculatus Say. 



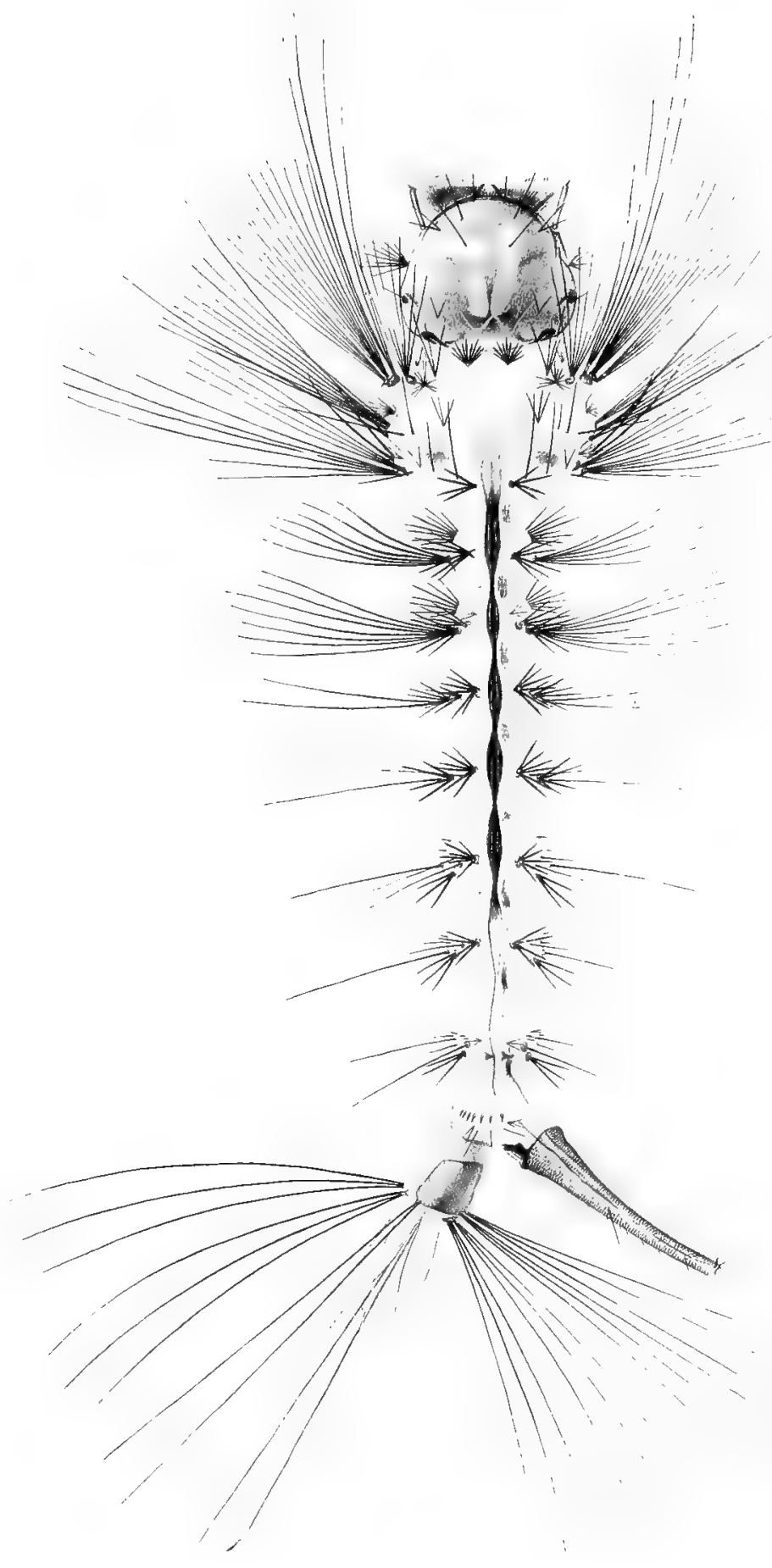

Larva: Sabethinus undosus Coquillett. 



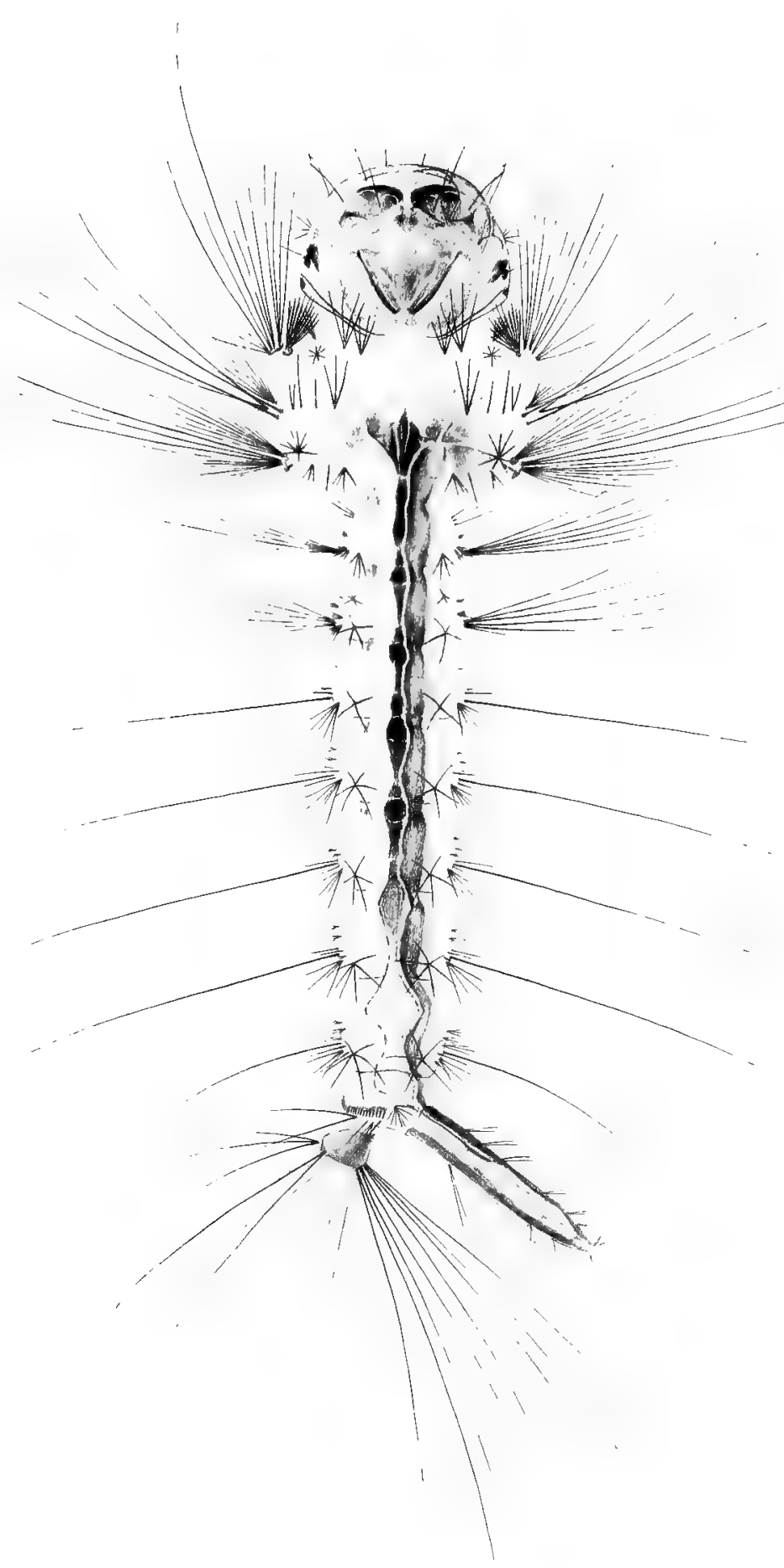

Larva: Wyeomyia vanduzeei Dyar \& Knab. 



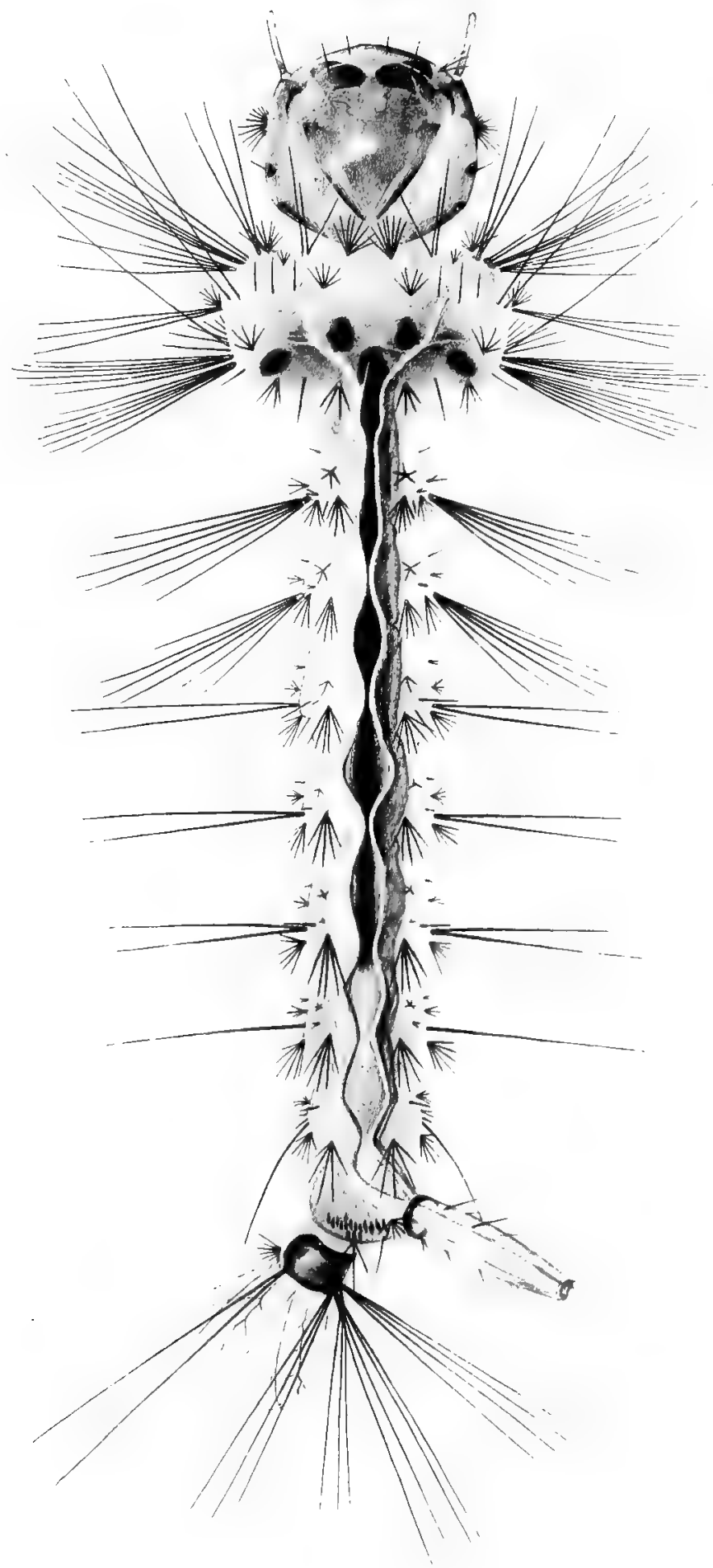

Larva: Wyeomyia aporonoma Dyar \& Knab. 



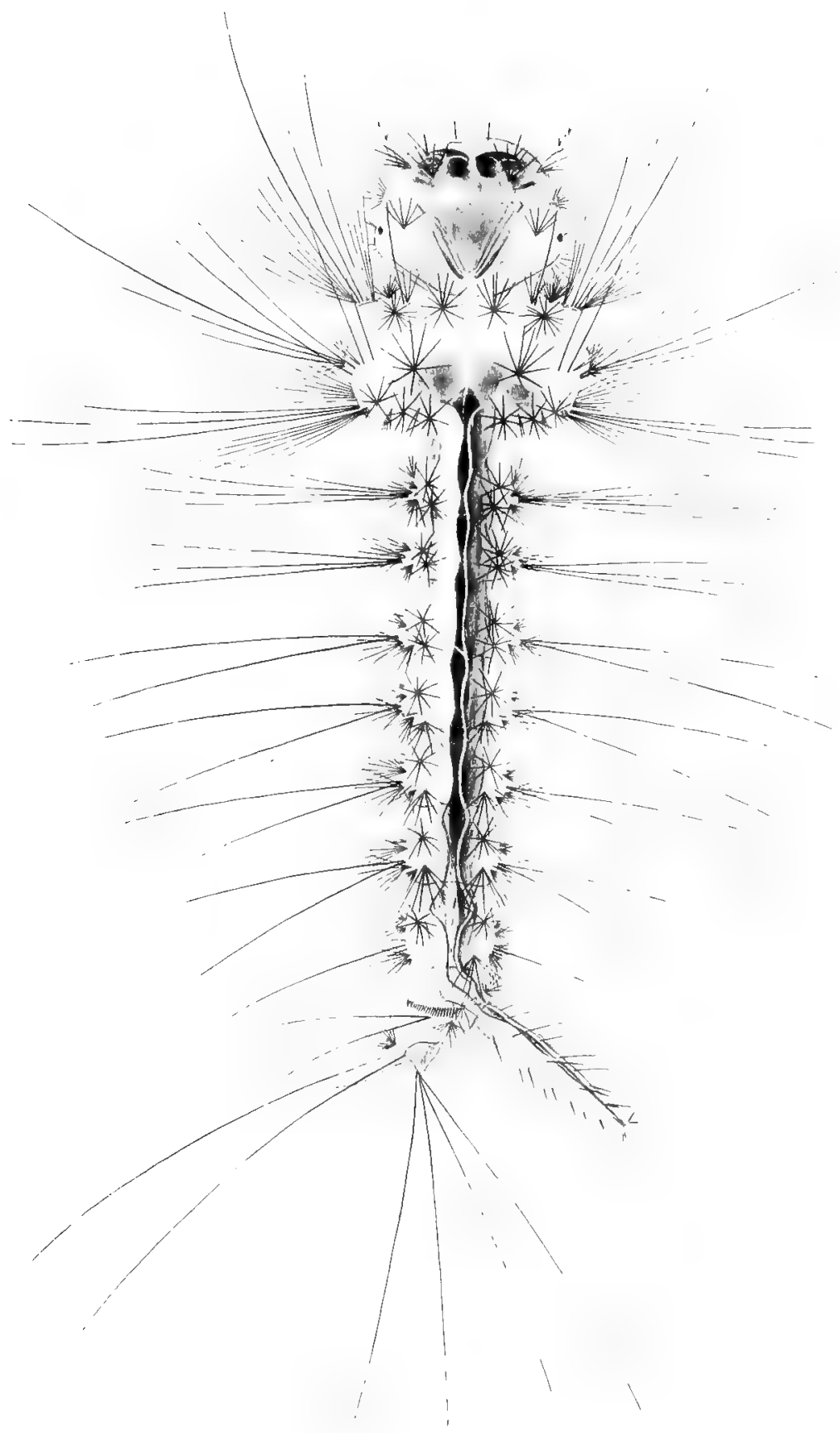





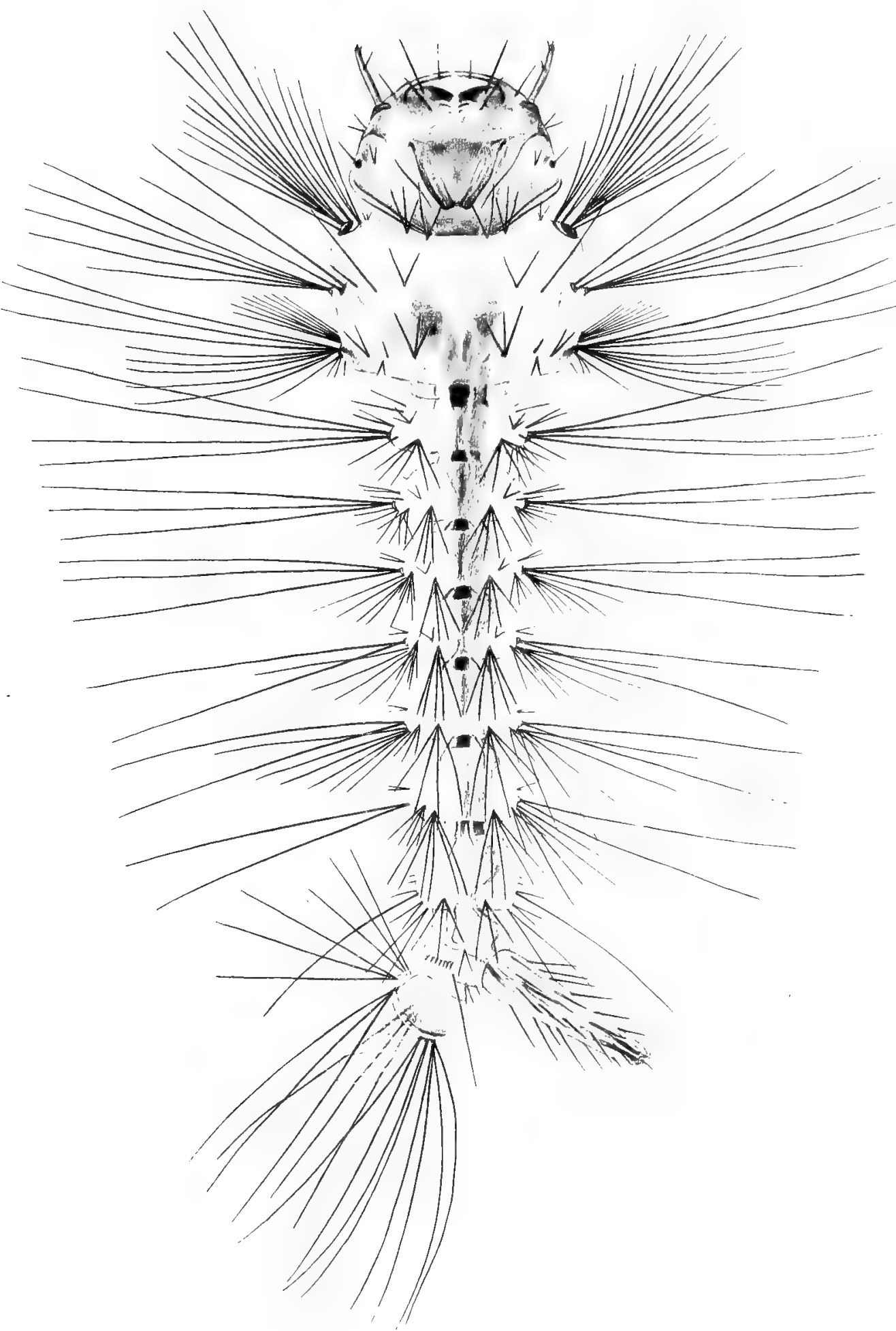





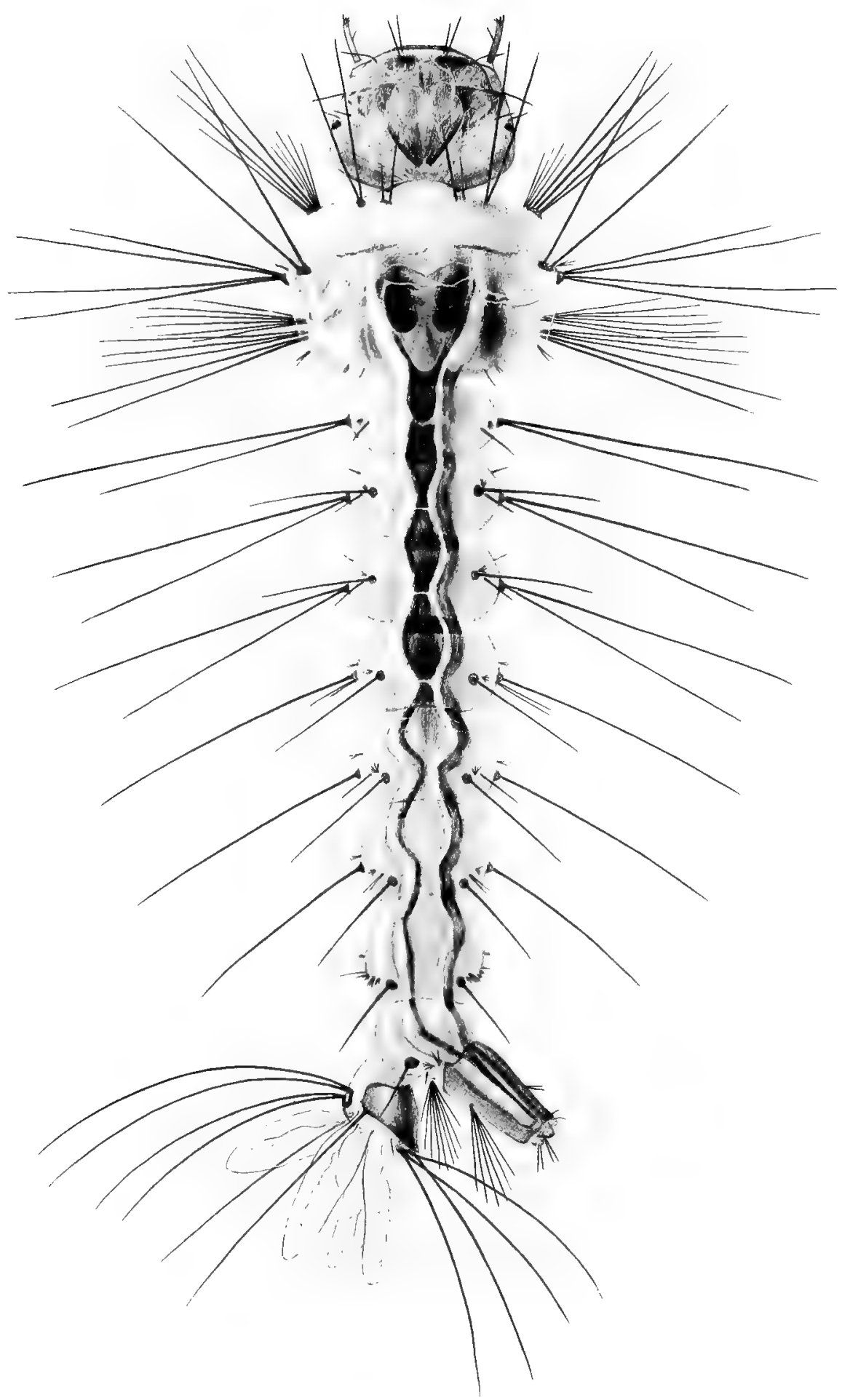

I.arra: Joblotia digitatus Rondani. 



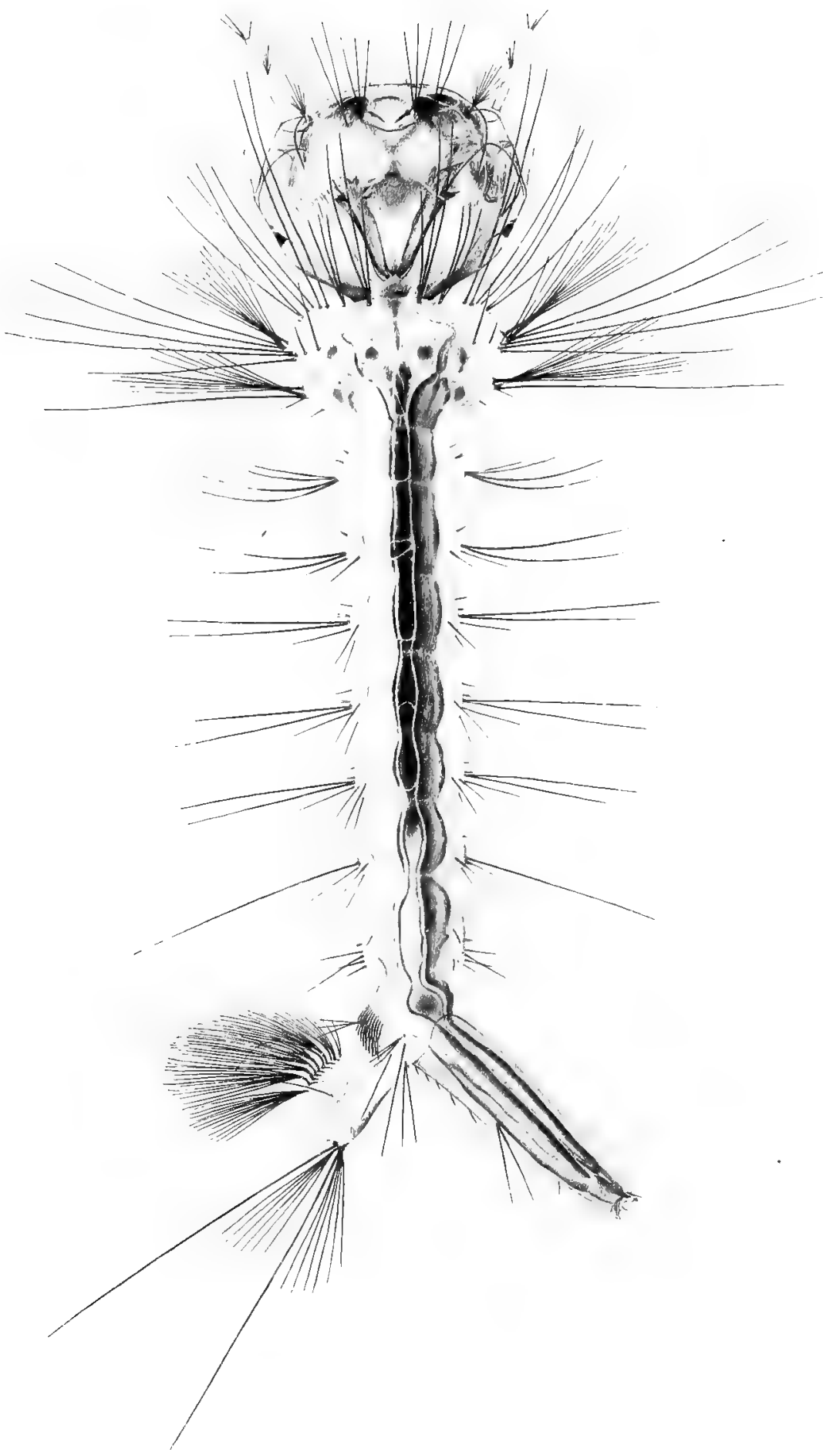

Iarva: Deinocerites cancer Thoobald. 



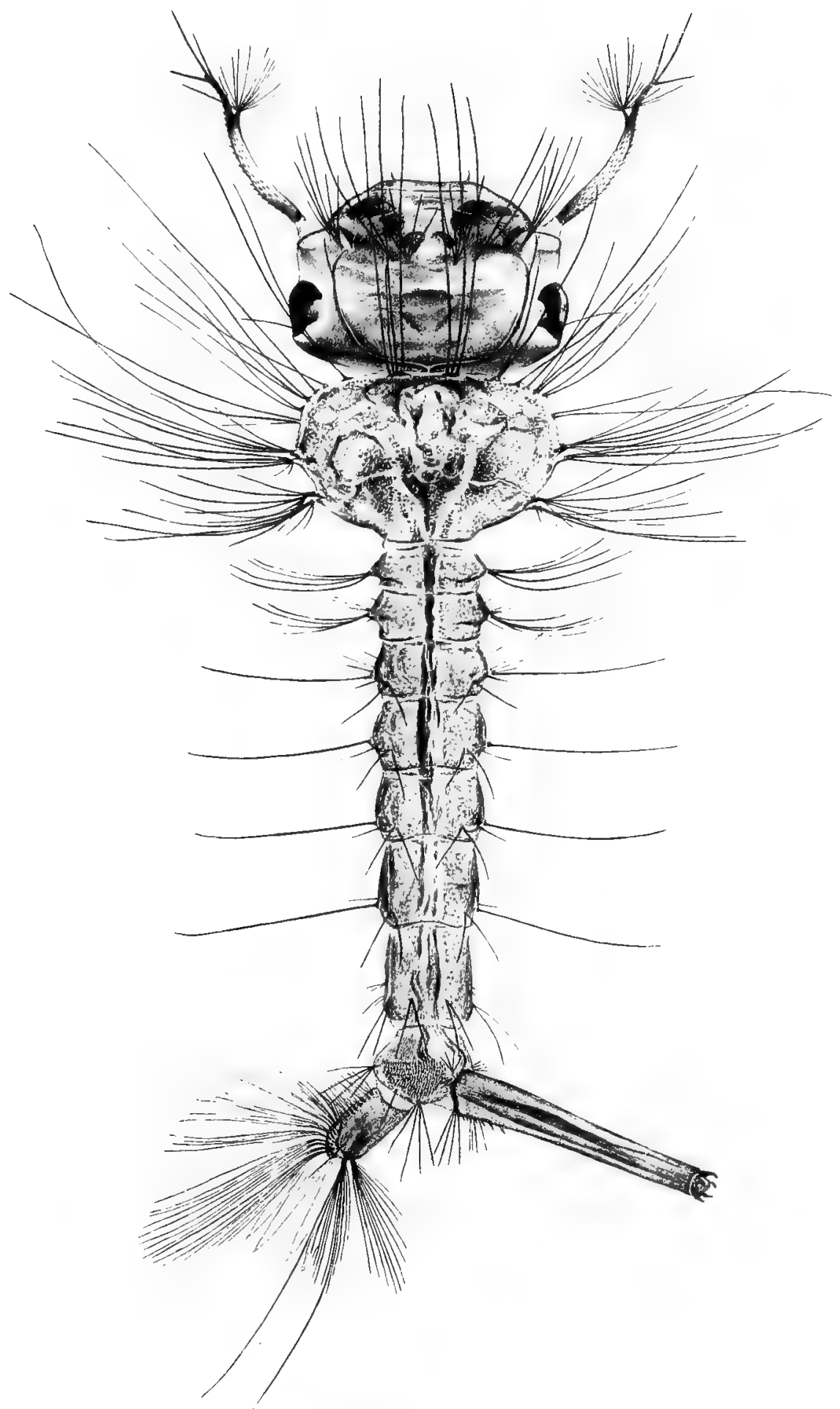

Larva: Culex dyari Coquillett. 

PUBLICATION 159

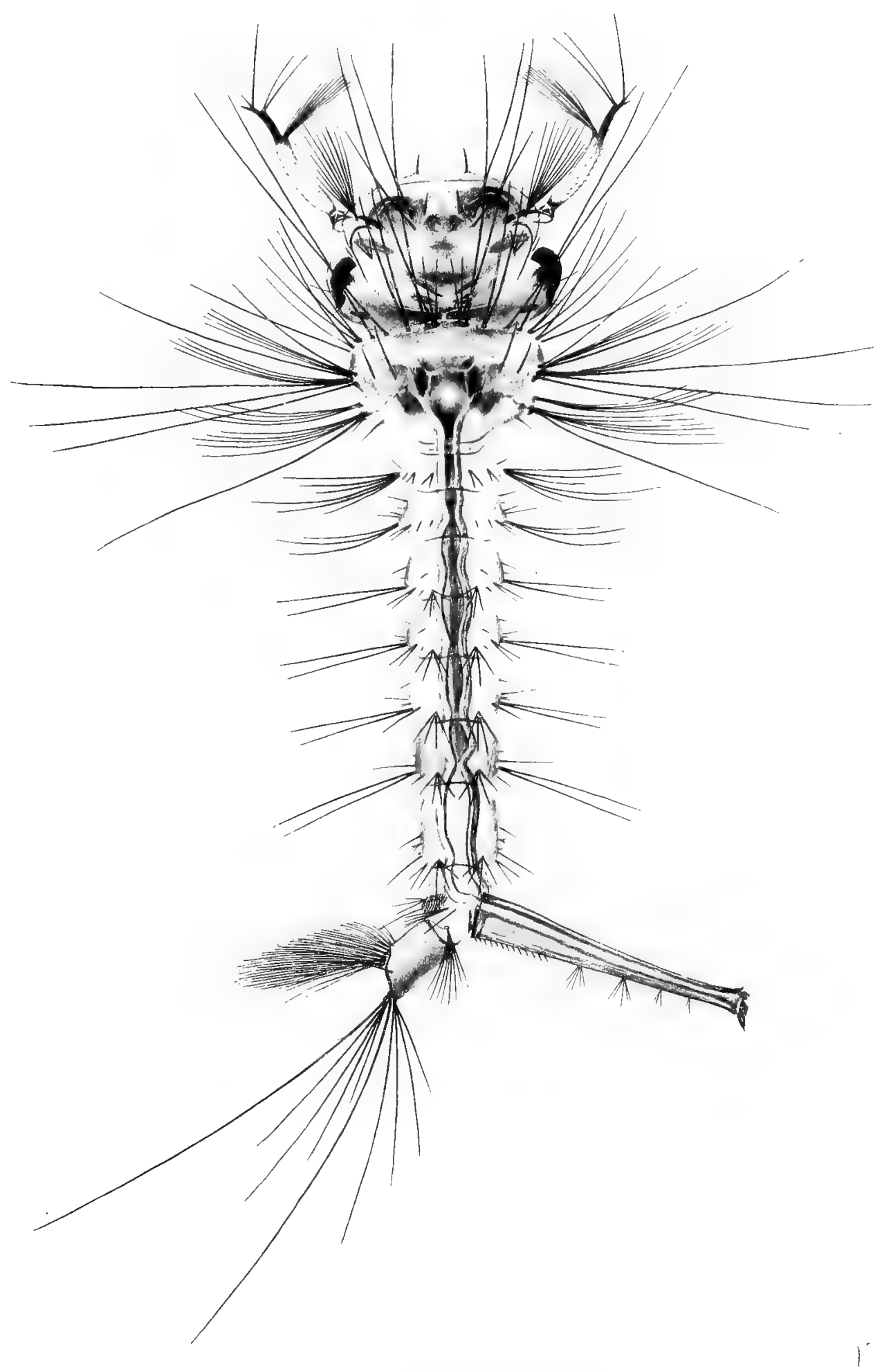

Larva: Culex territans Walkor. 



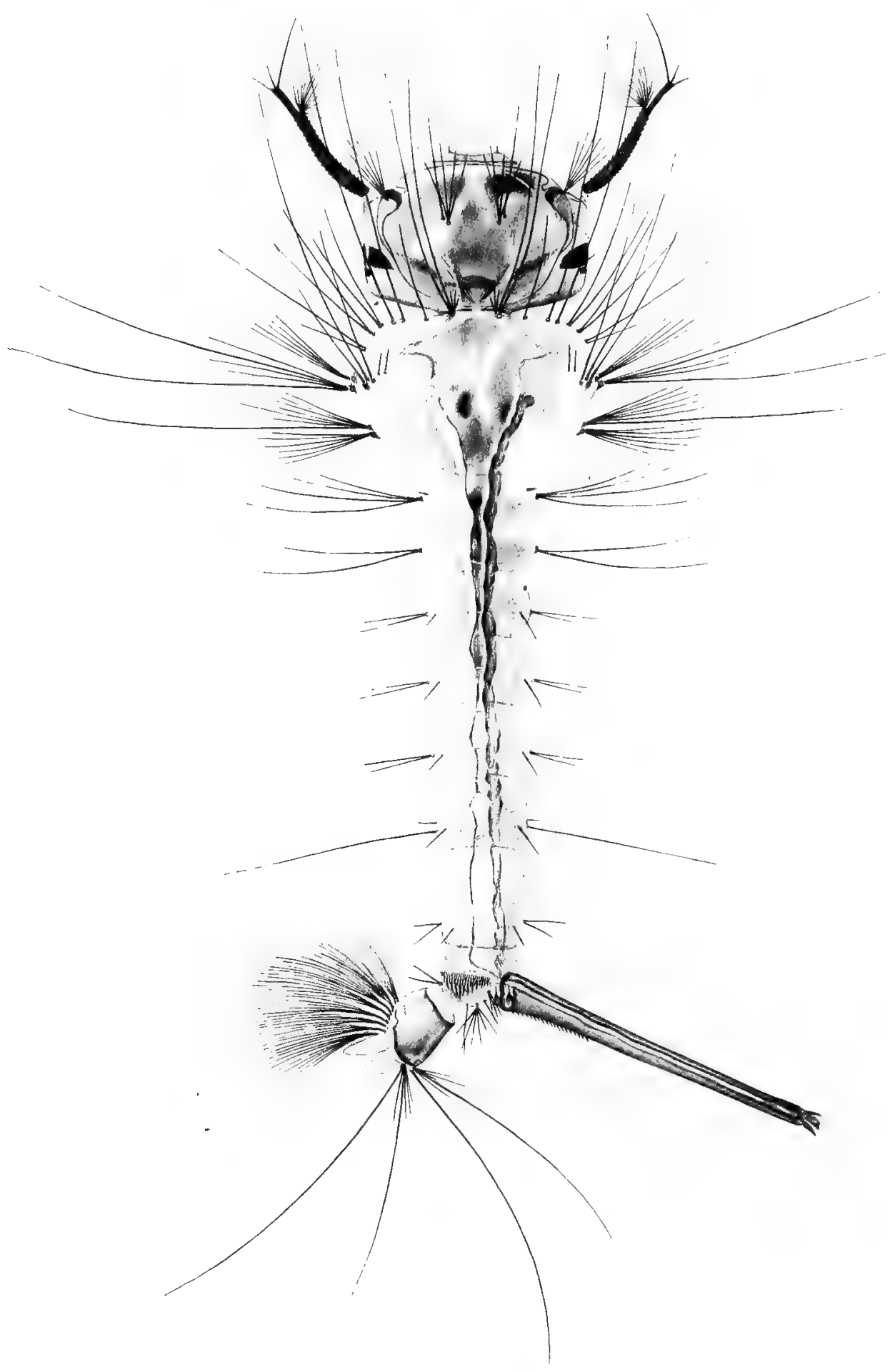

Larva: Culex latisquamma Coquillett. 

PUBLICATION 159

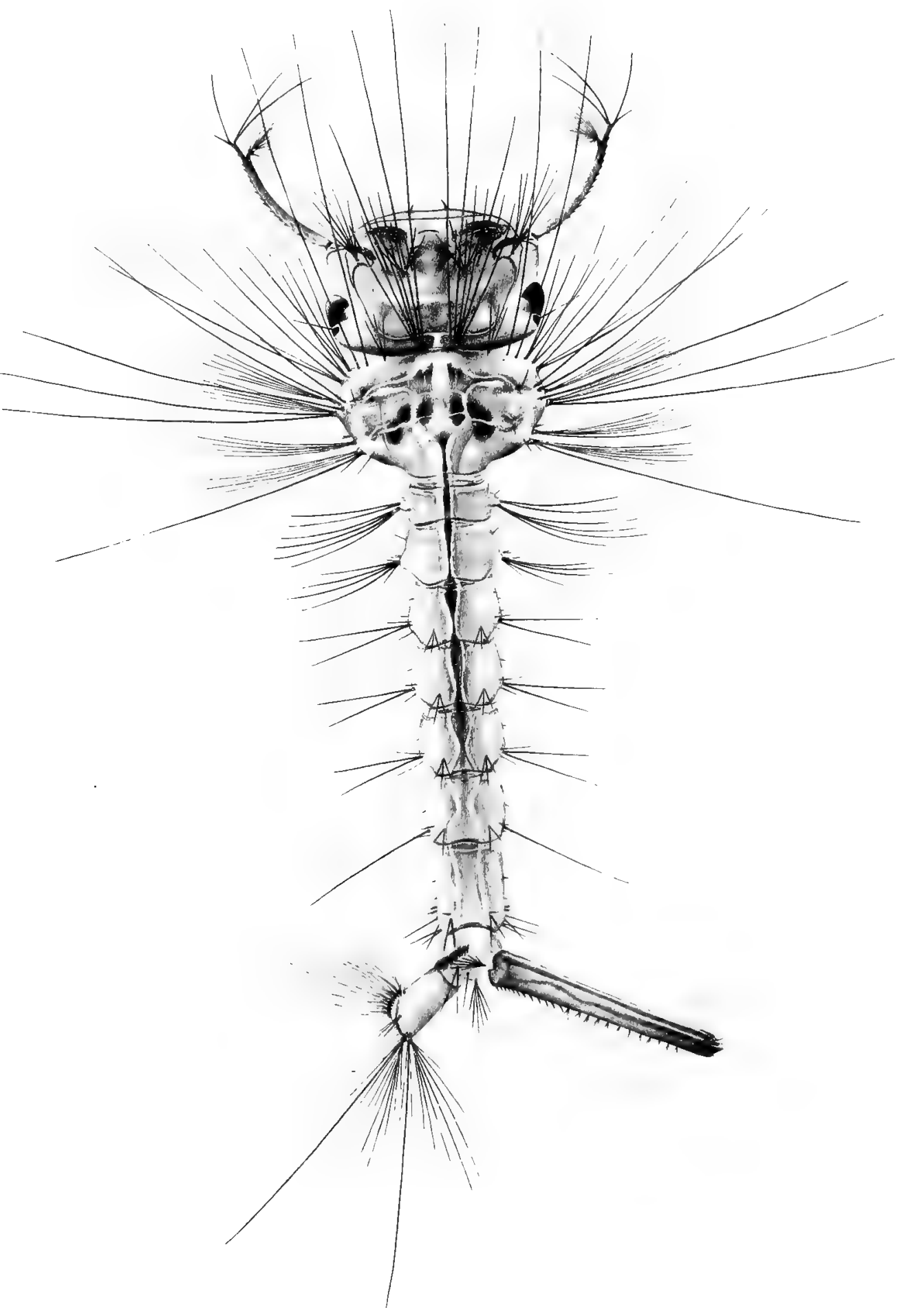

Larva: Culex melanurus Coquillett. 



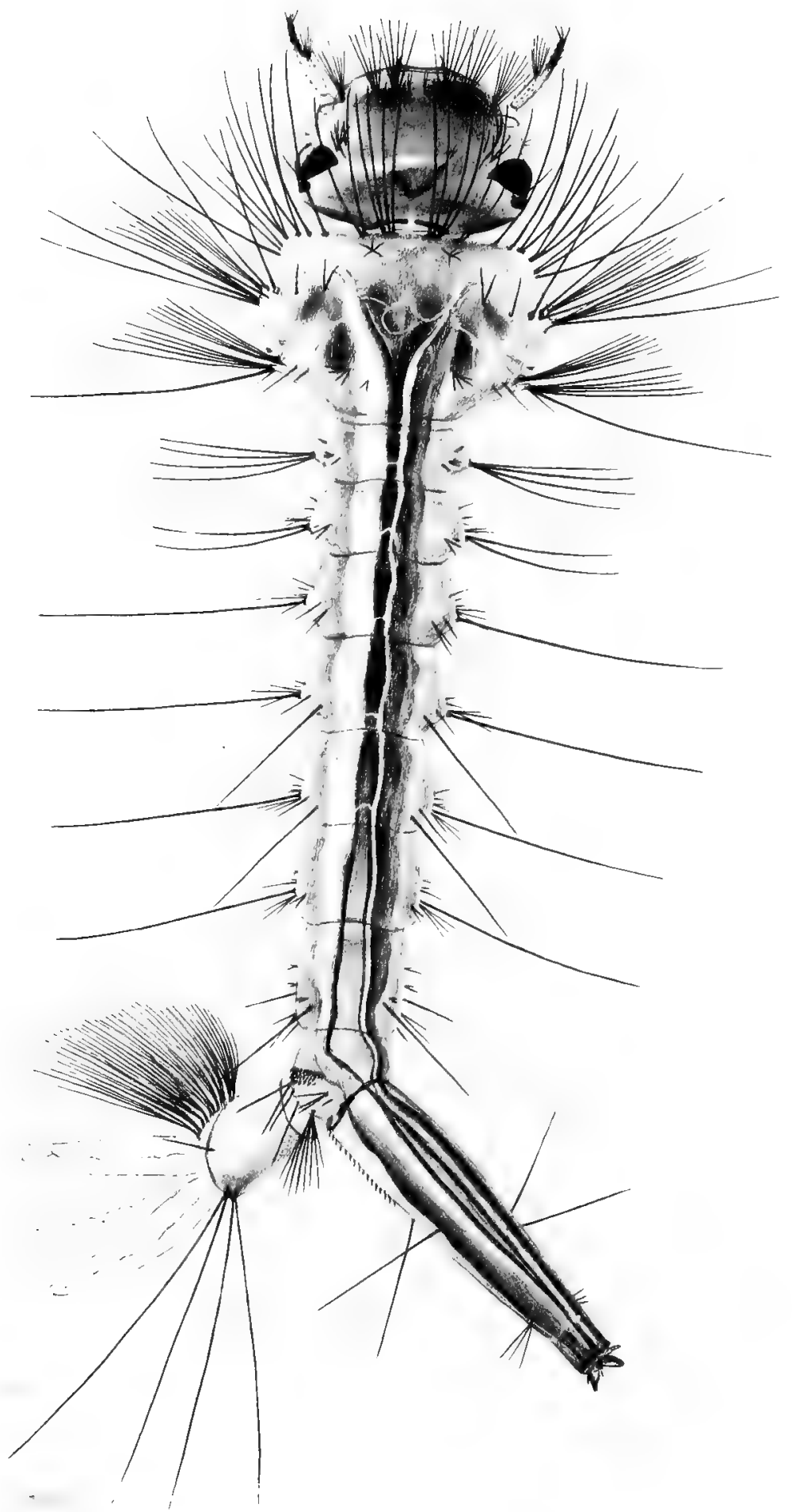

Larva: Culex restuans Thcobald. 



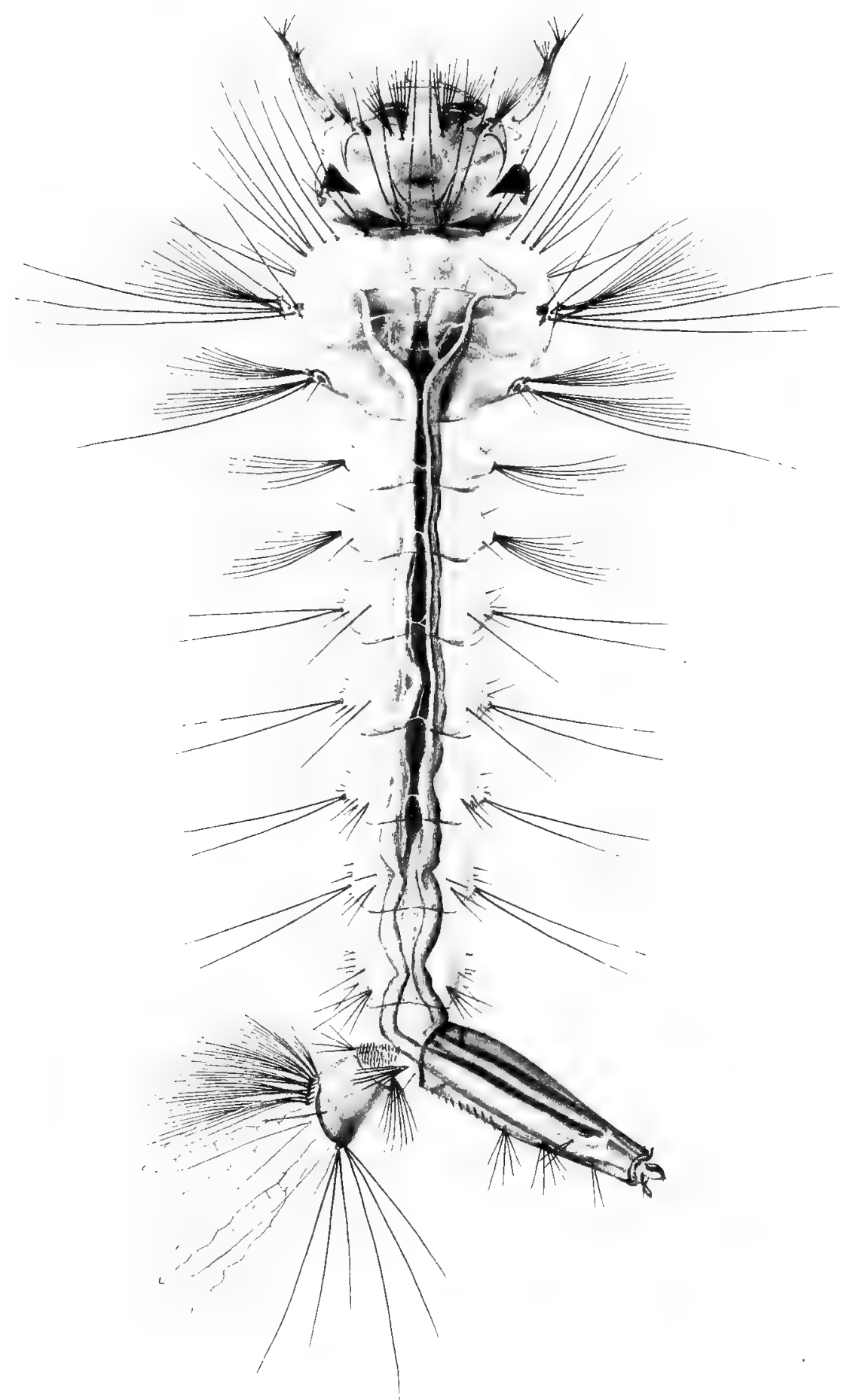





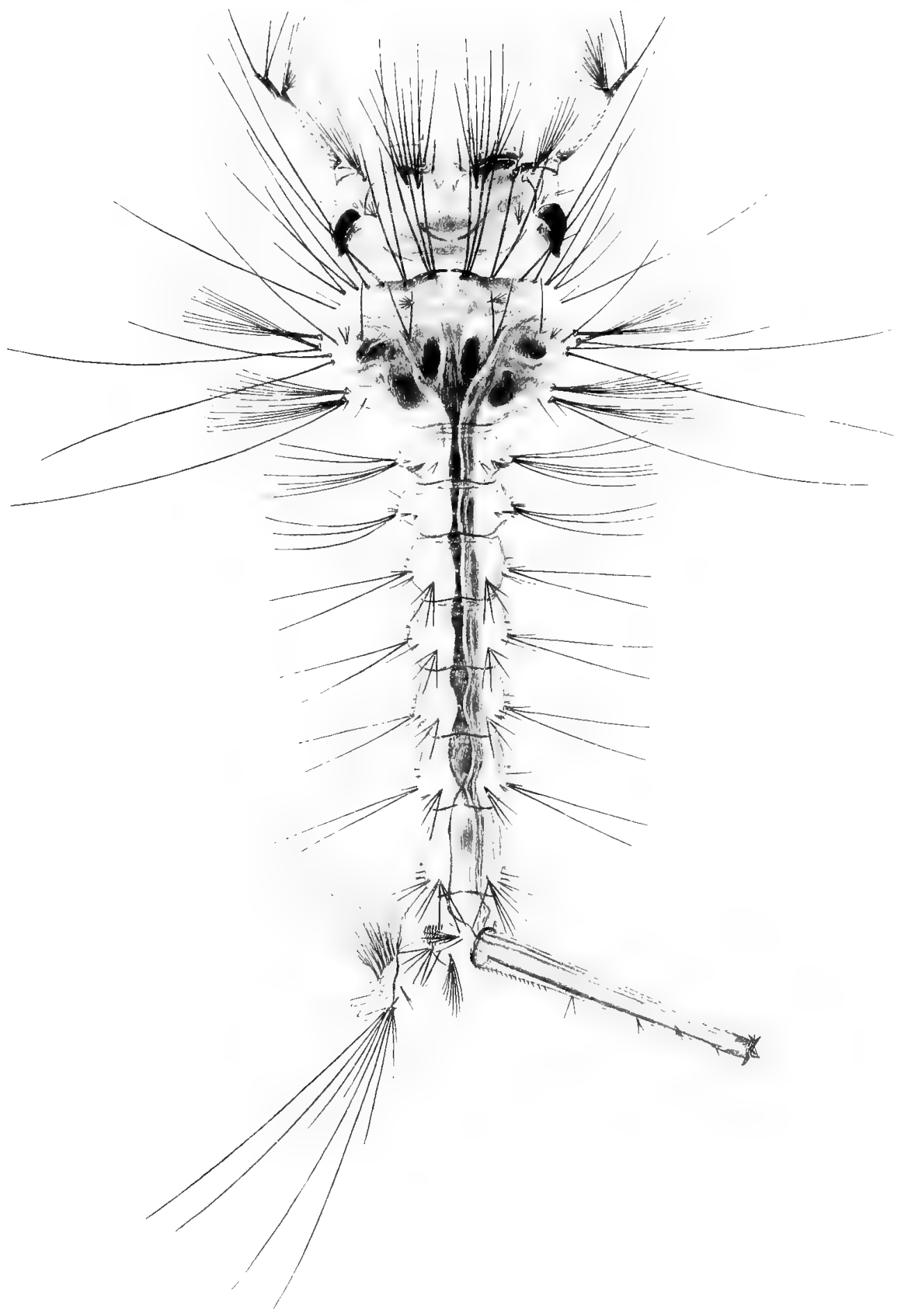

Larva: Culex salinarius Coquillett. 



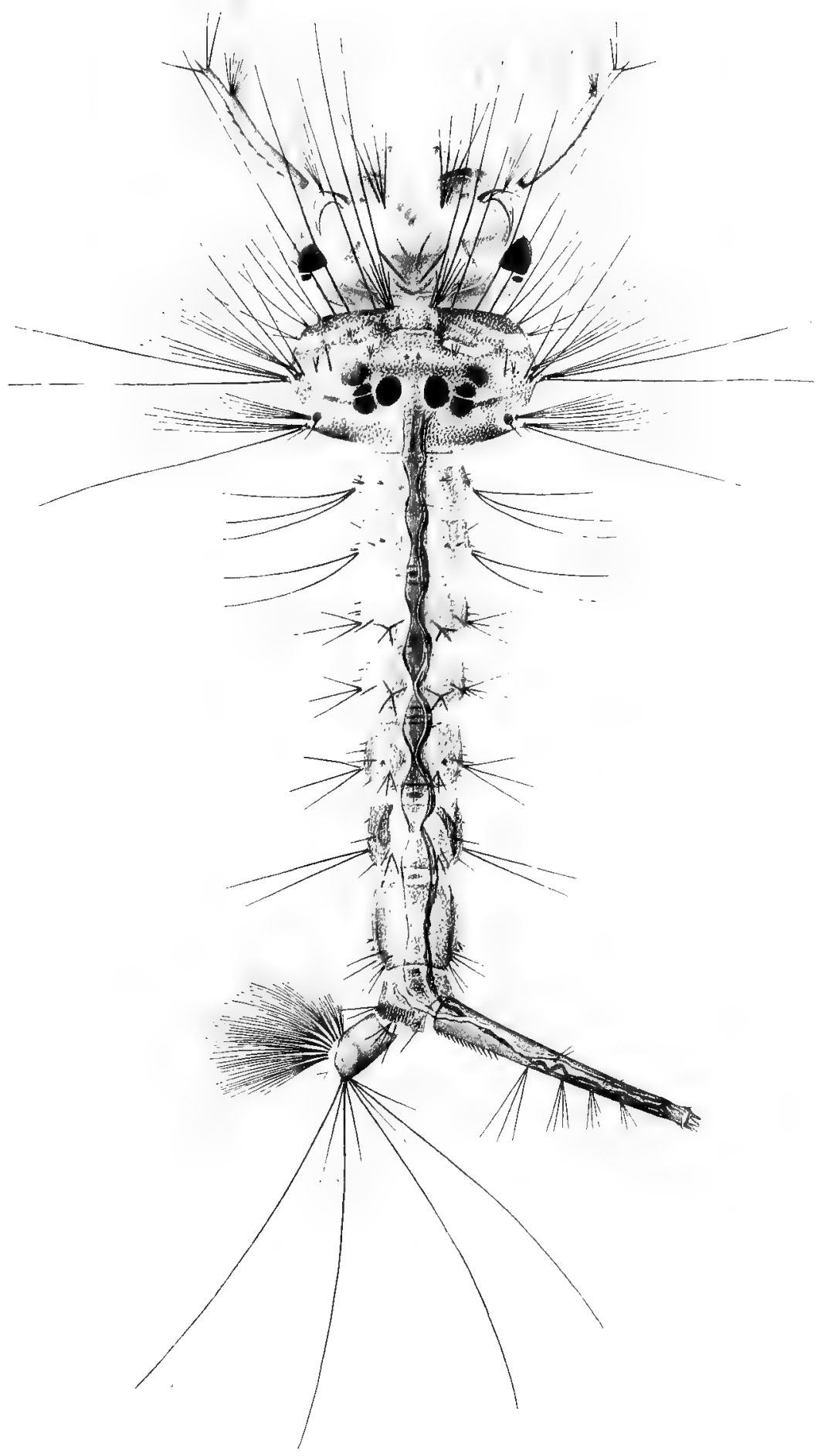

Larva: Culex decorator Dyar \& Knab. 



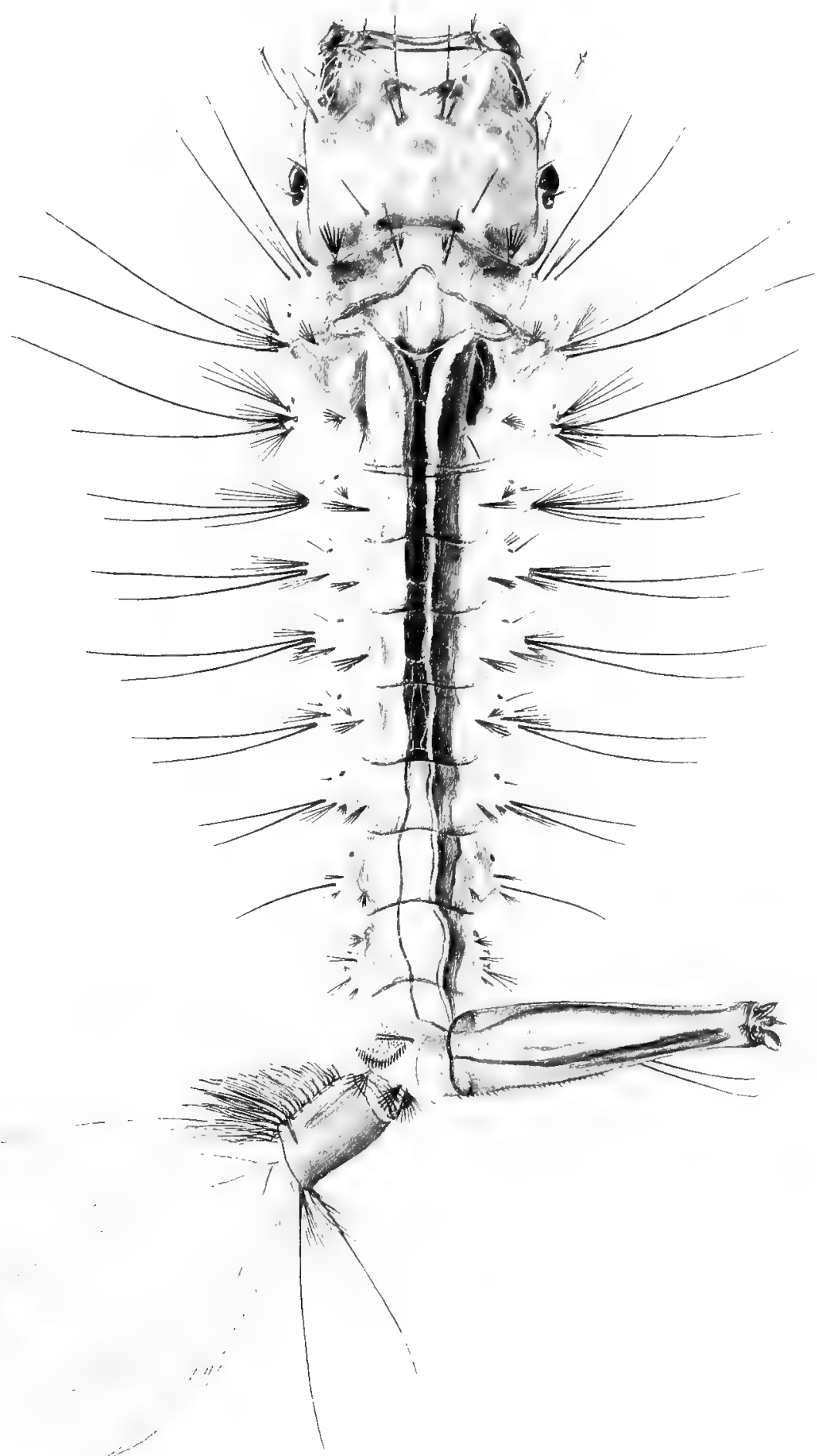




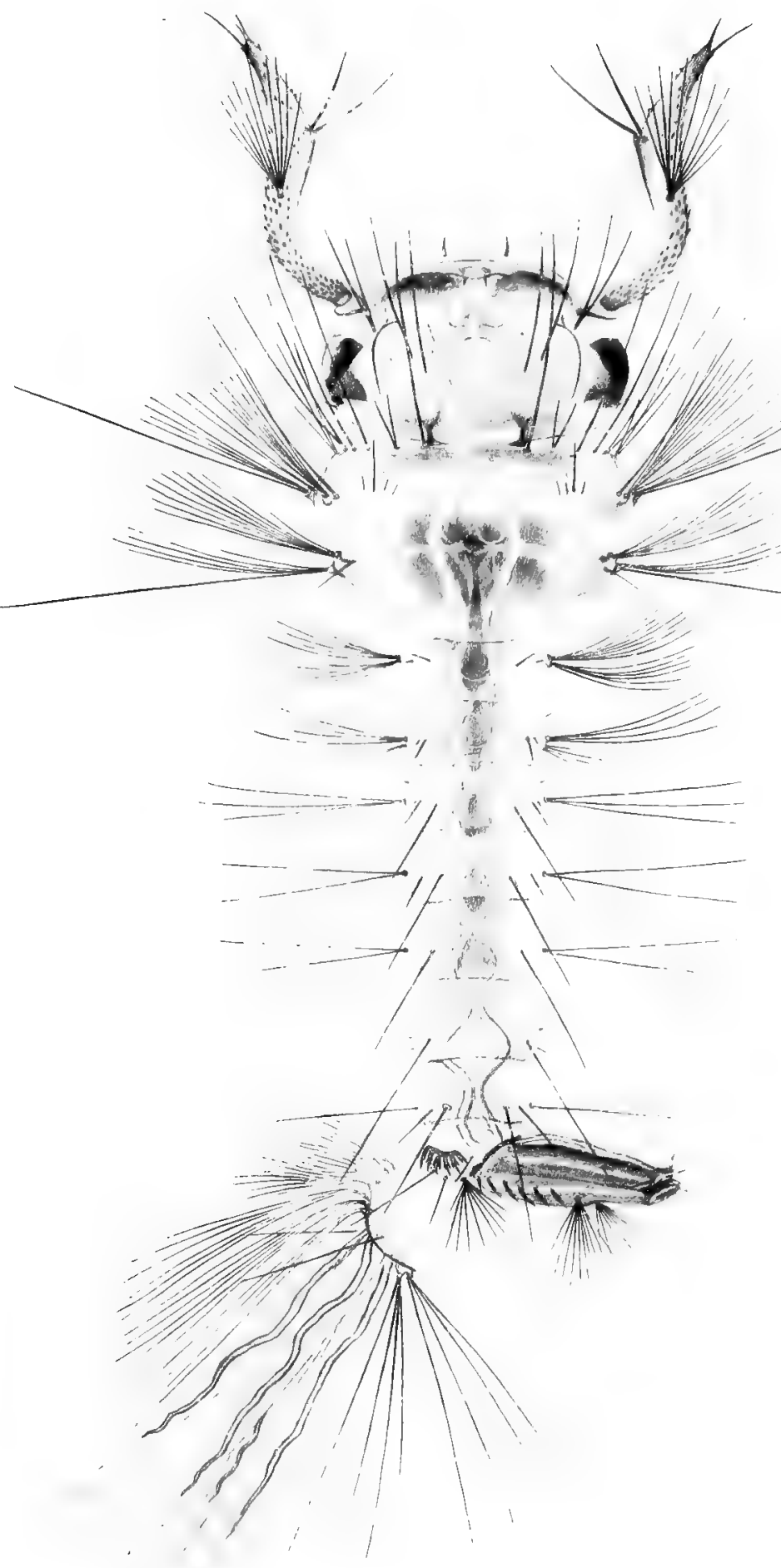

Larva: Psorophora discolor Coquillert. 



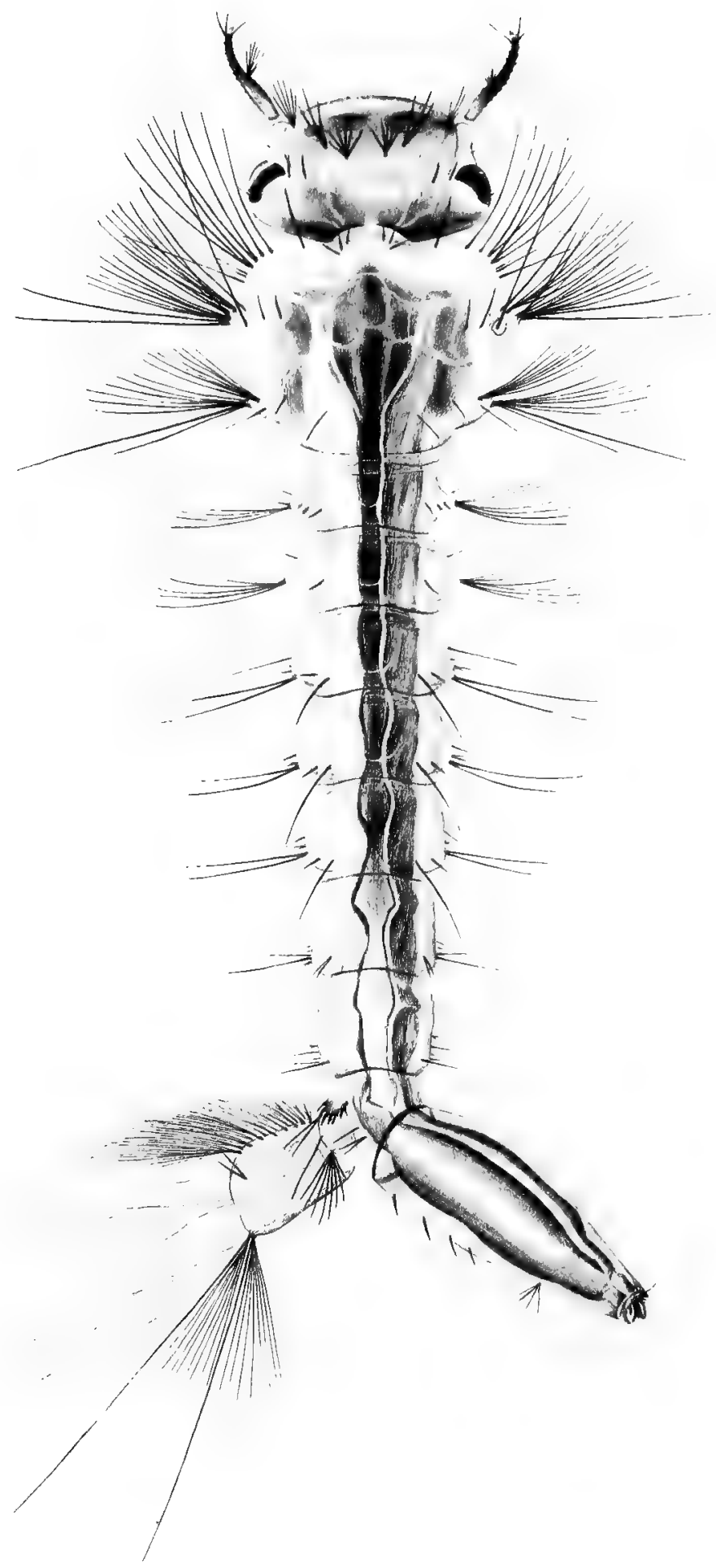

Larva: Psorophora columbiæ Dyar \& Knab. 



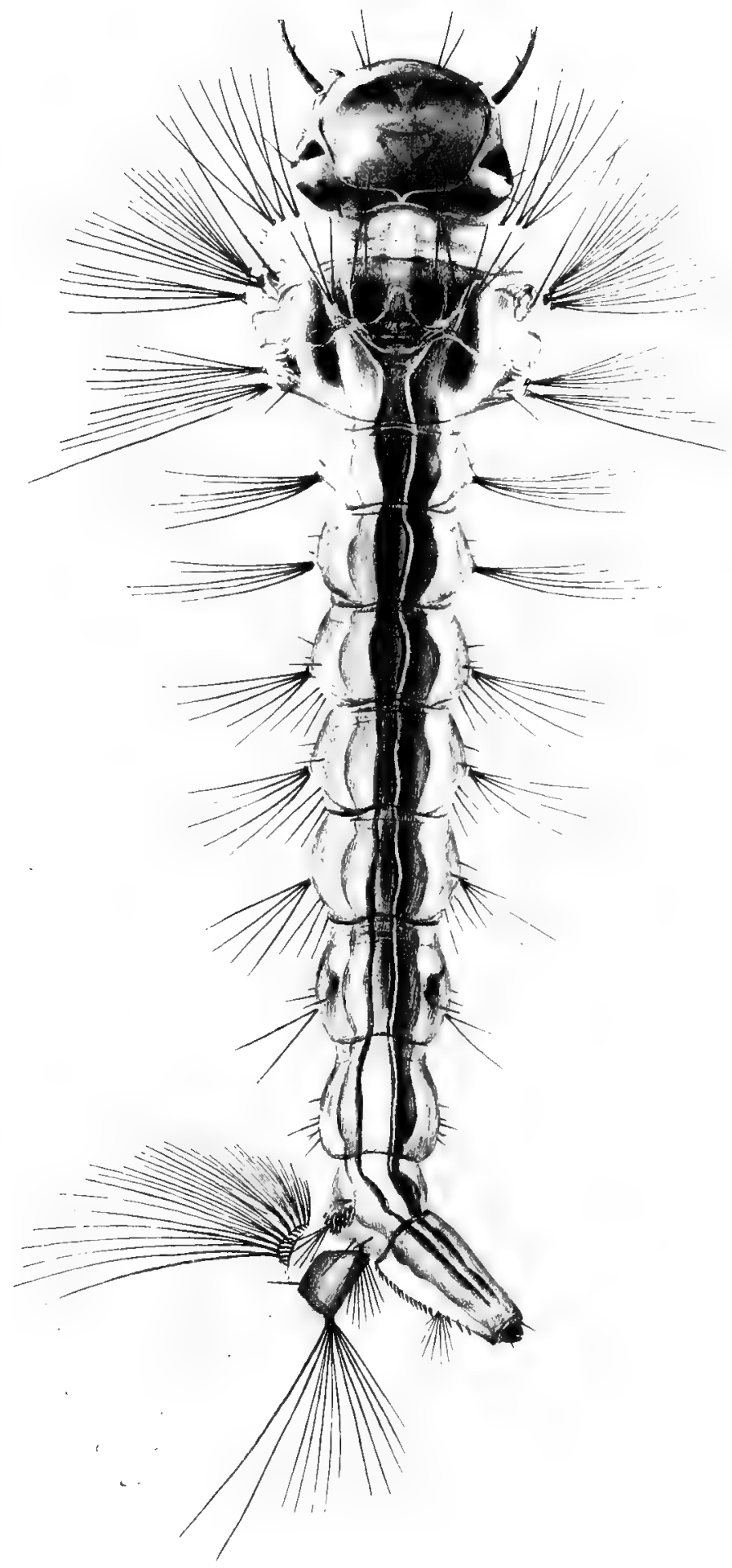

Larva: Aedes atropalpus Coquillett. 



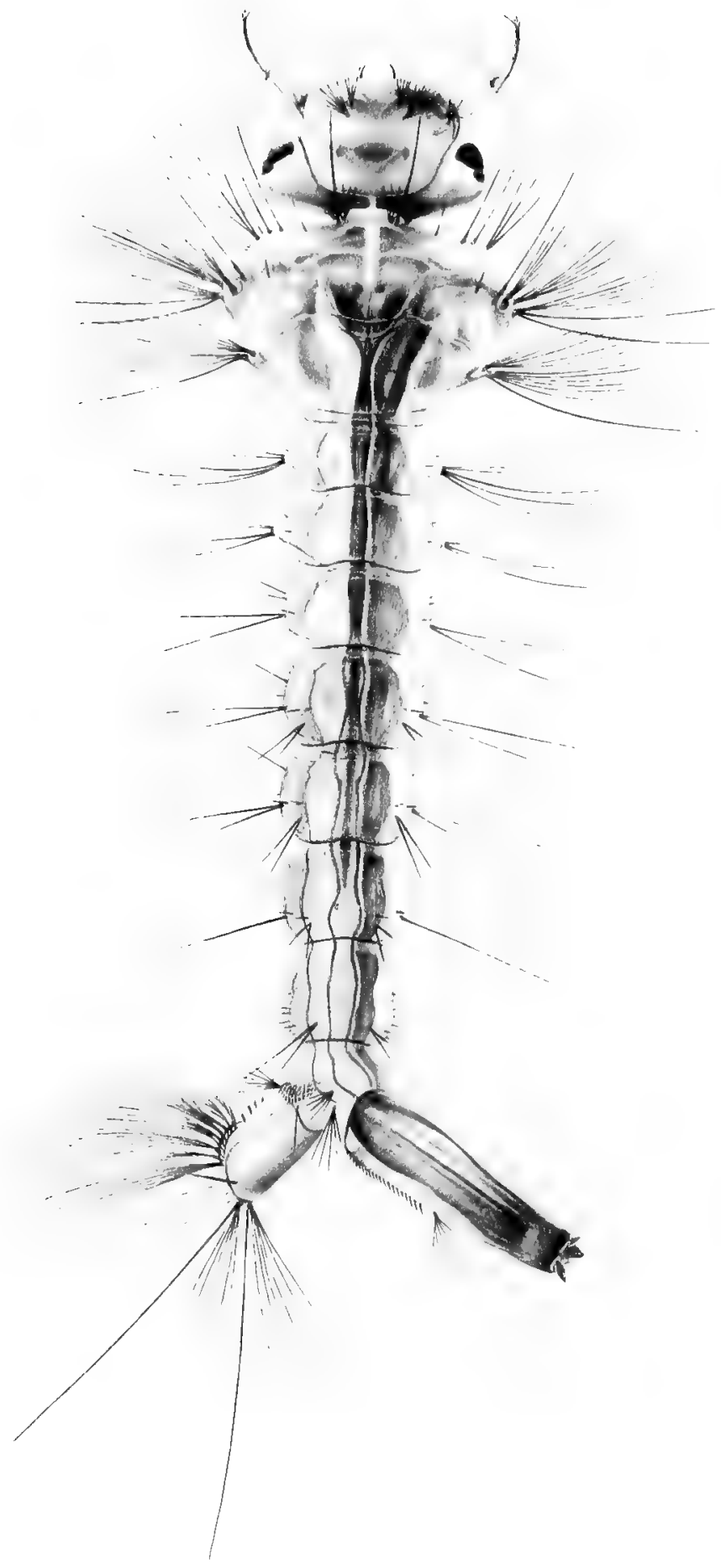

Larva: Aedes canadensis Theobald. 



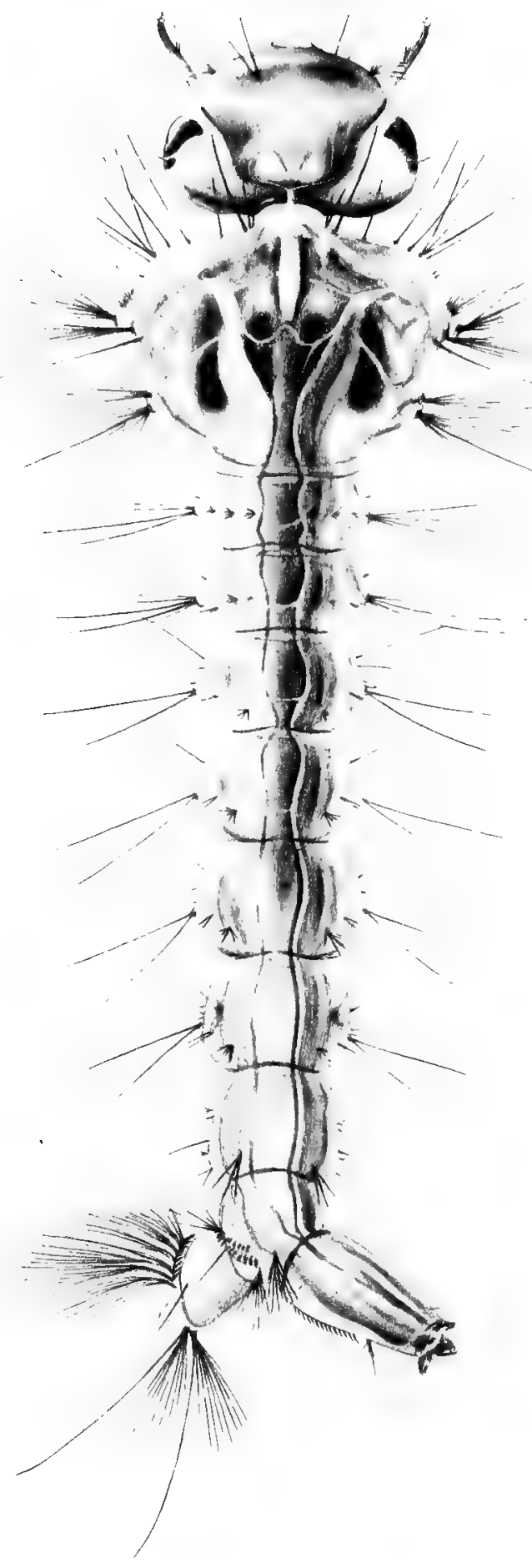

Larva: Aedes sollicitans Walker. 



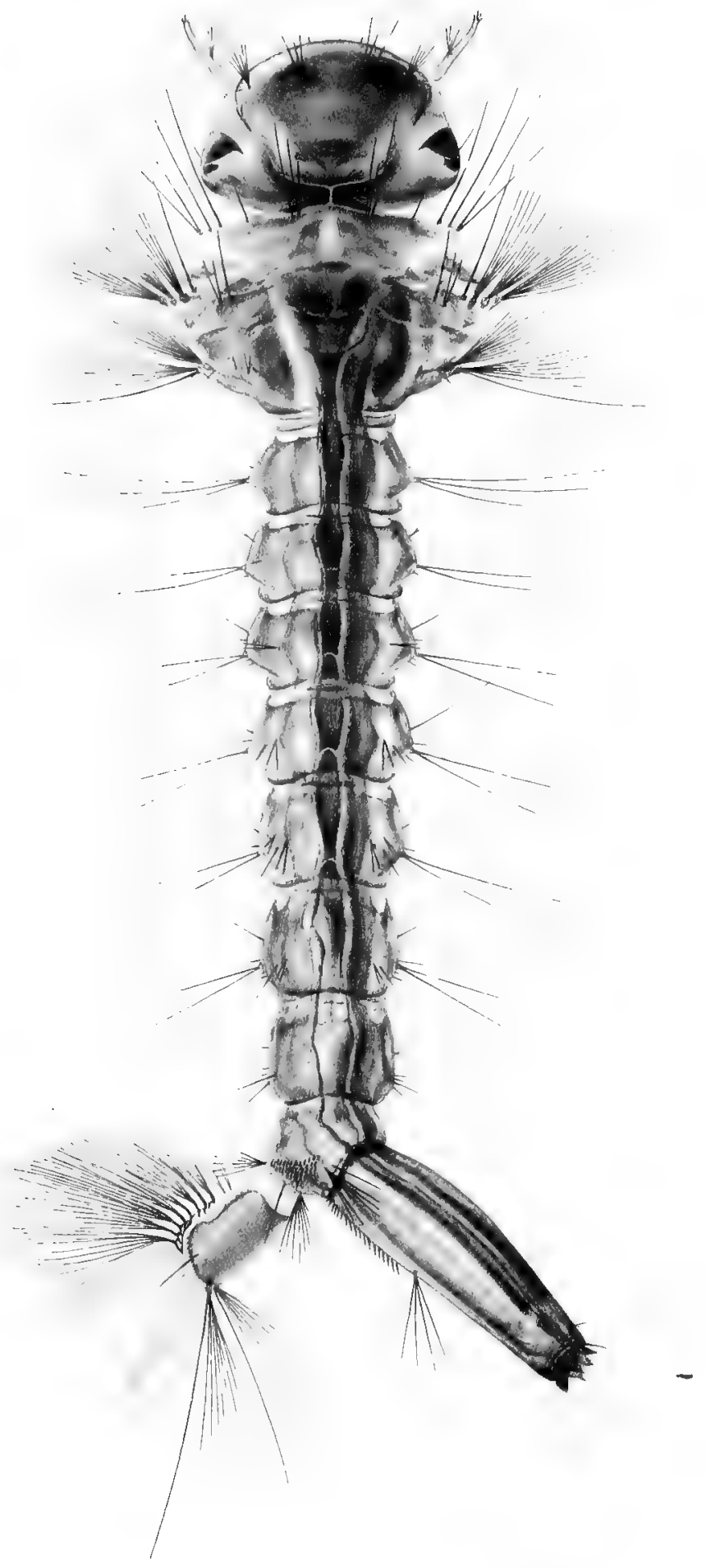

Larva: Aedes stimulans Walker. 



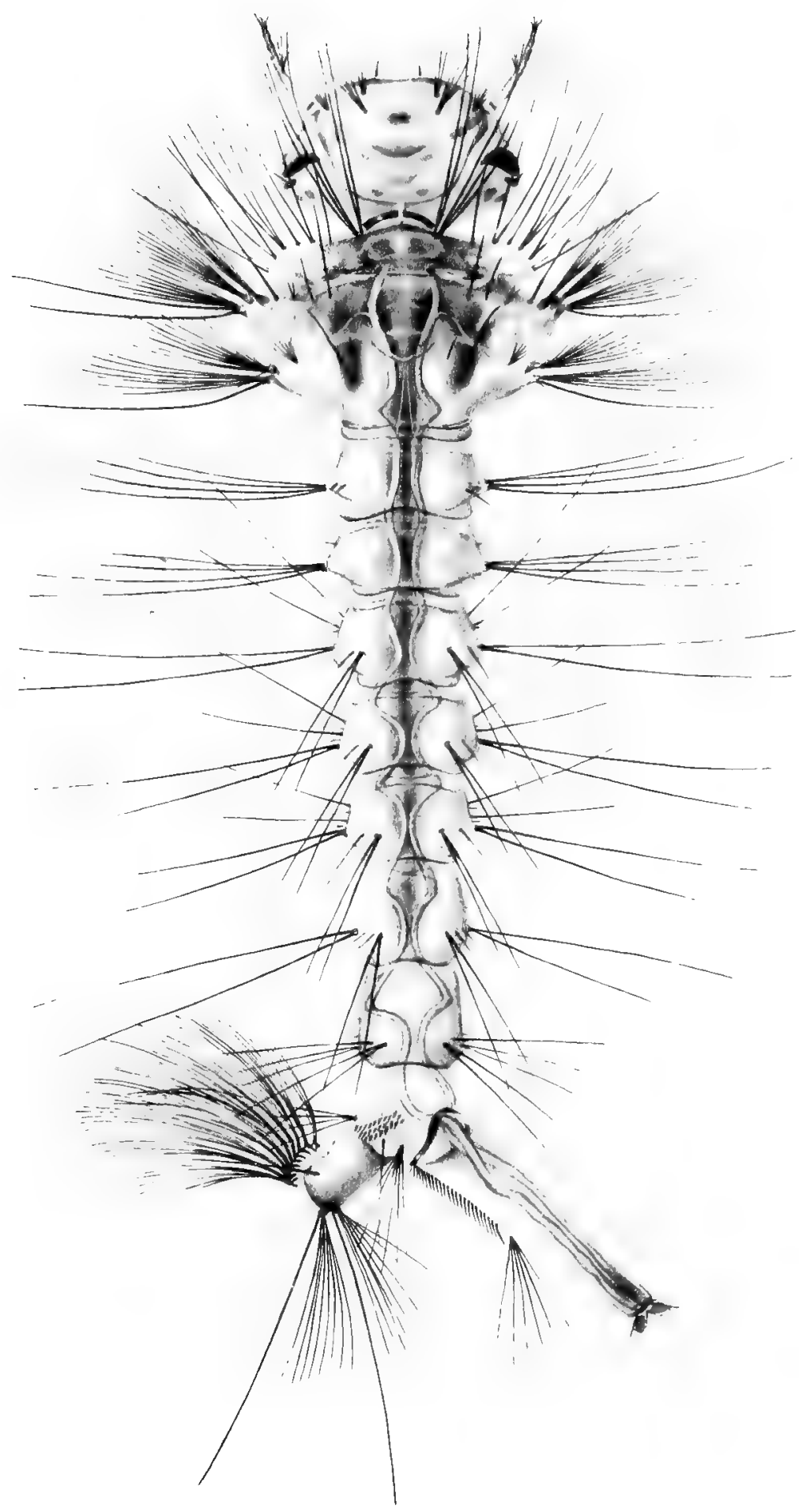

Larva: Aedes fitchii Felt $\mathcal{E}$ Young. 



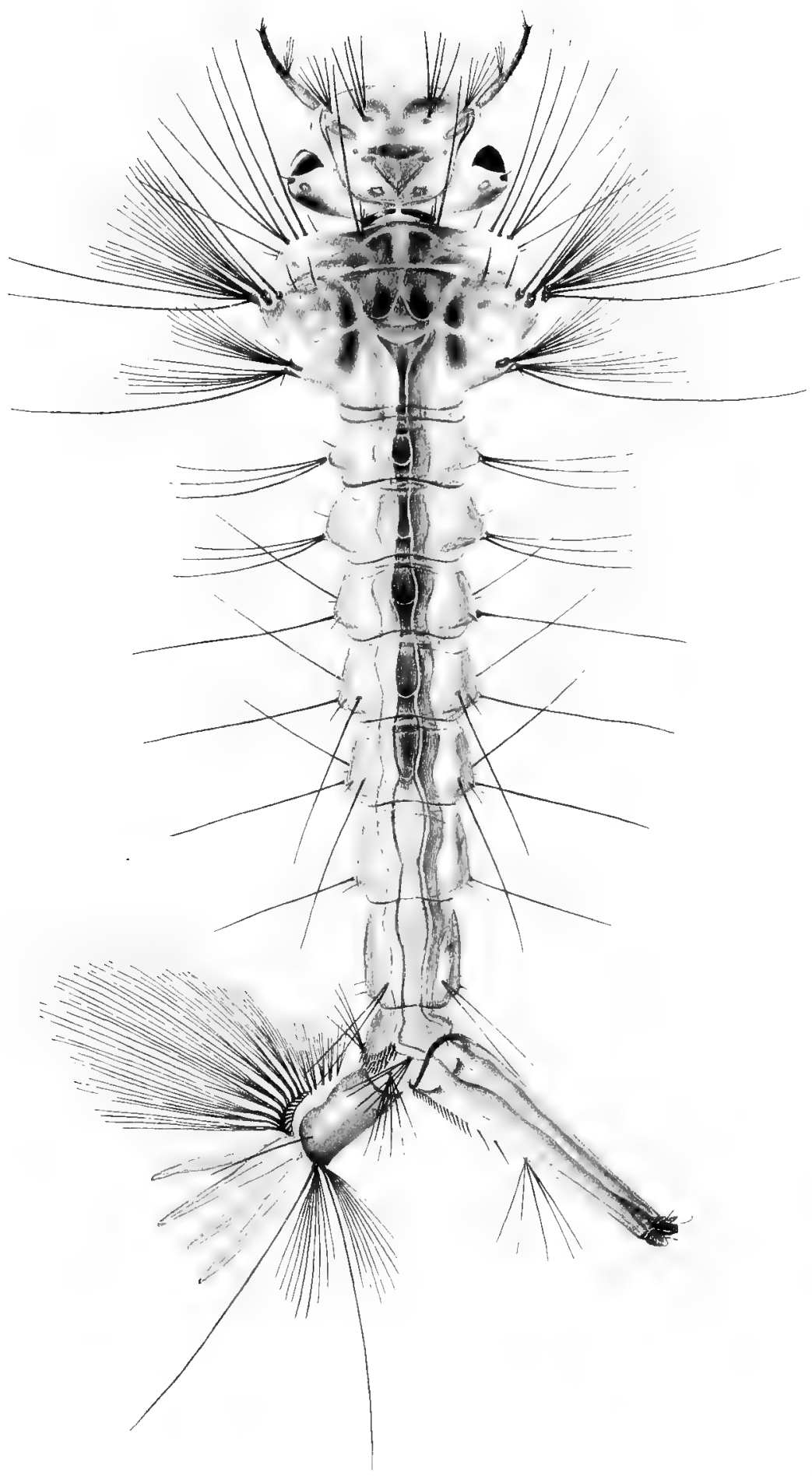

Larva: Aedes abfitchii Felt. 



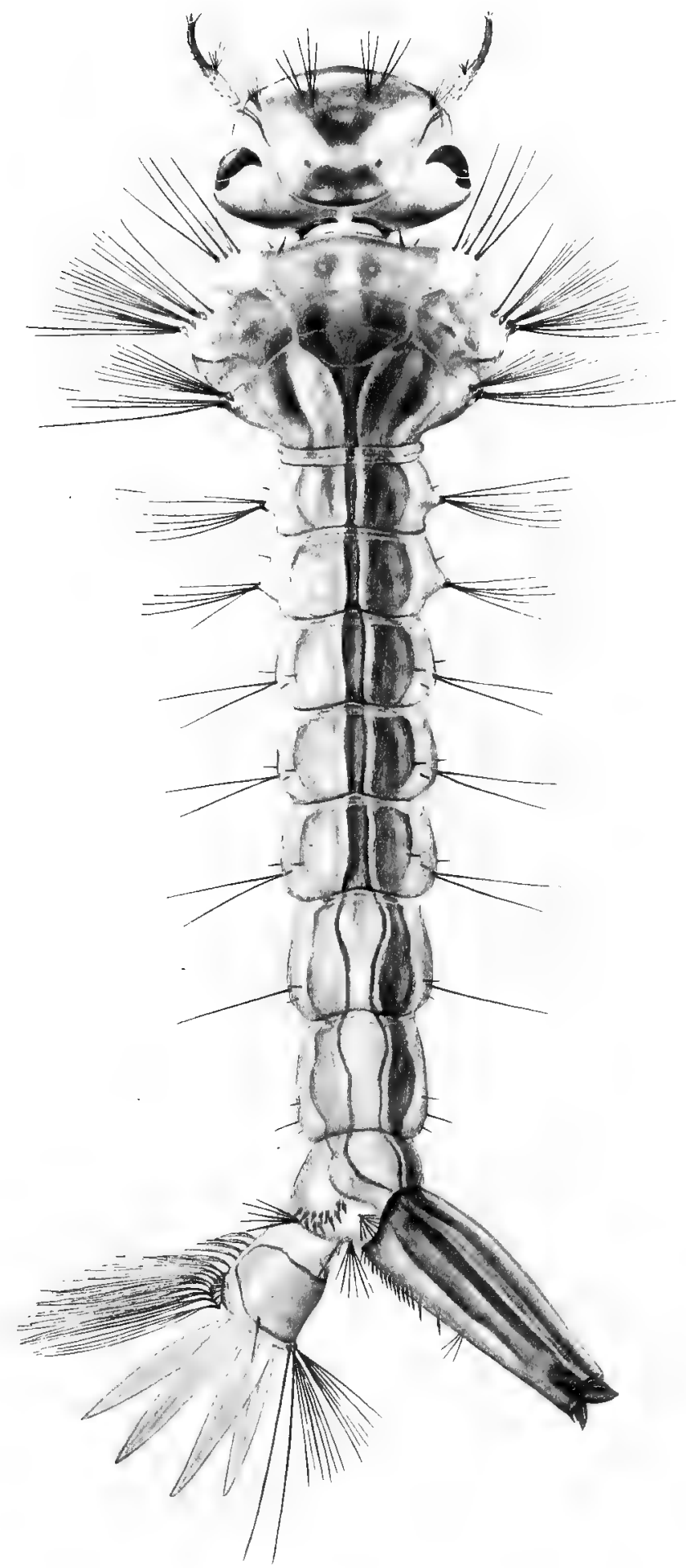

Larva: Apdes sylvestris Thenbald. 



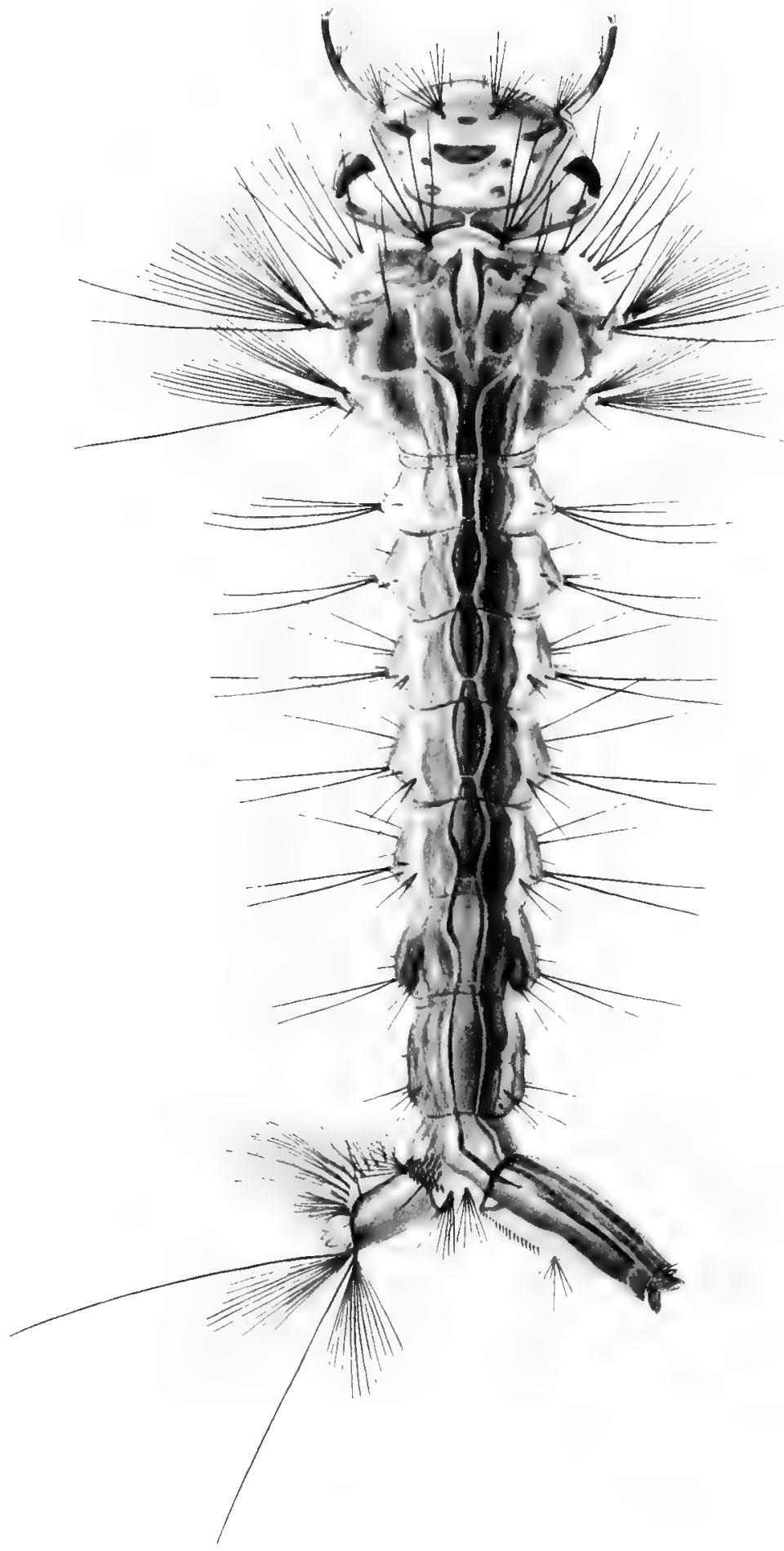

Larva: Aeries cantator Coquilletl. 



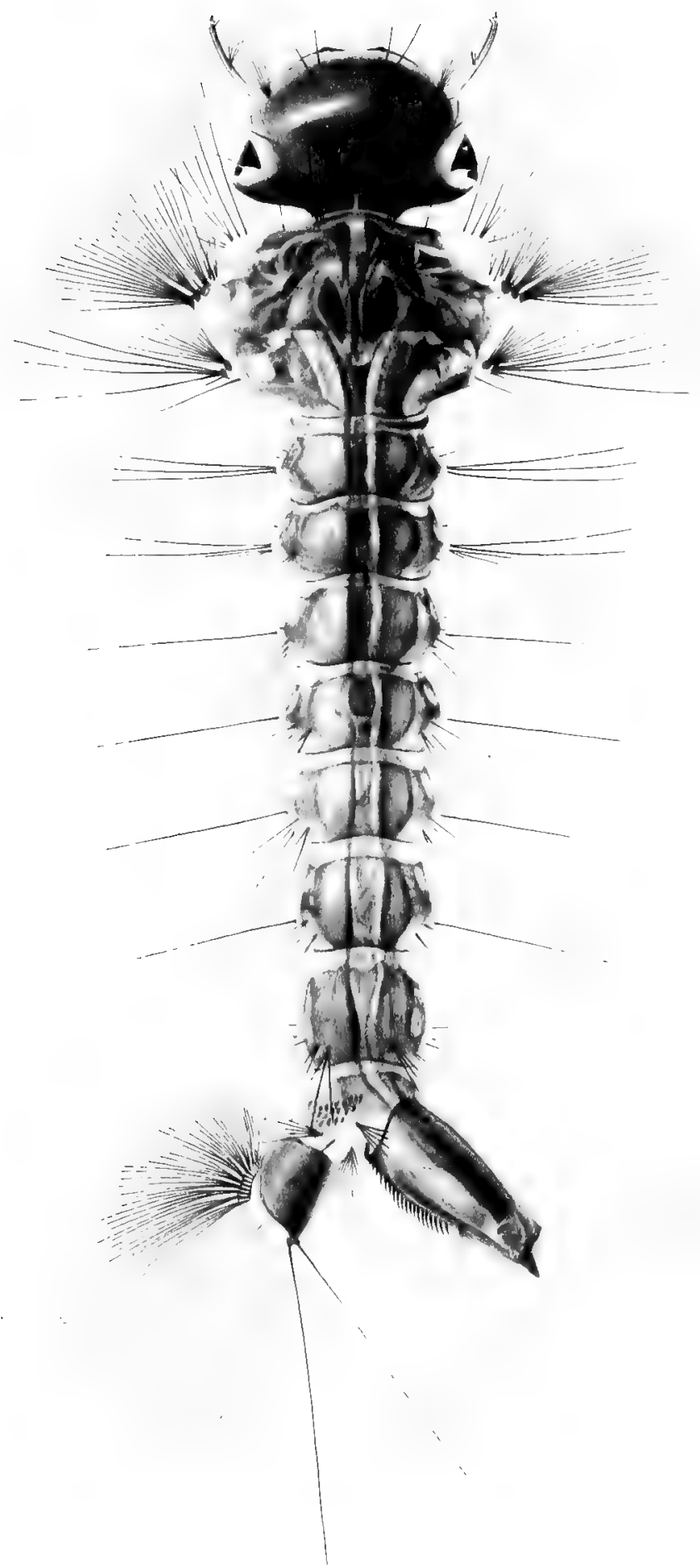

Larvid: Aedes trivithatus Comillett. 



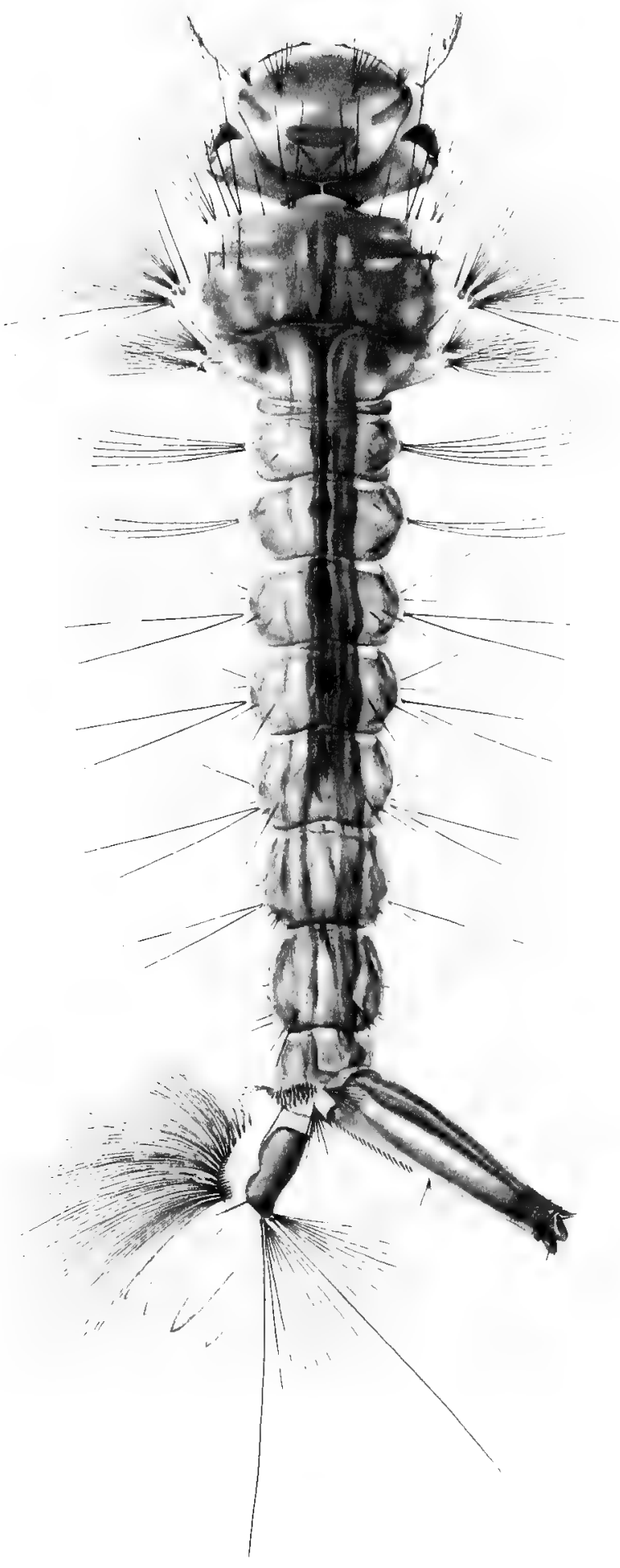

larva: Aedes giossbecki l'yar Knah. 



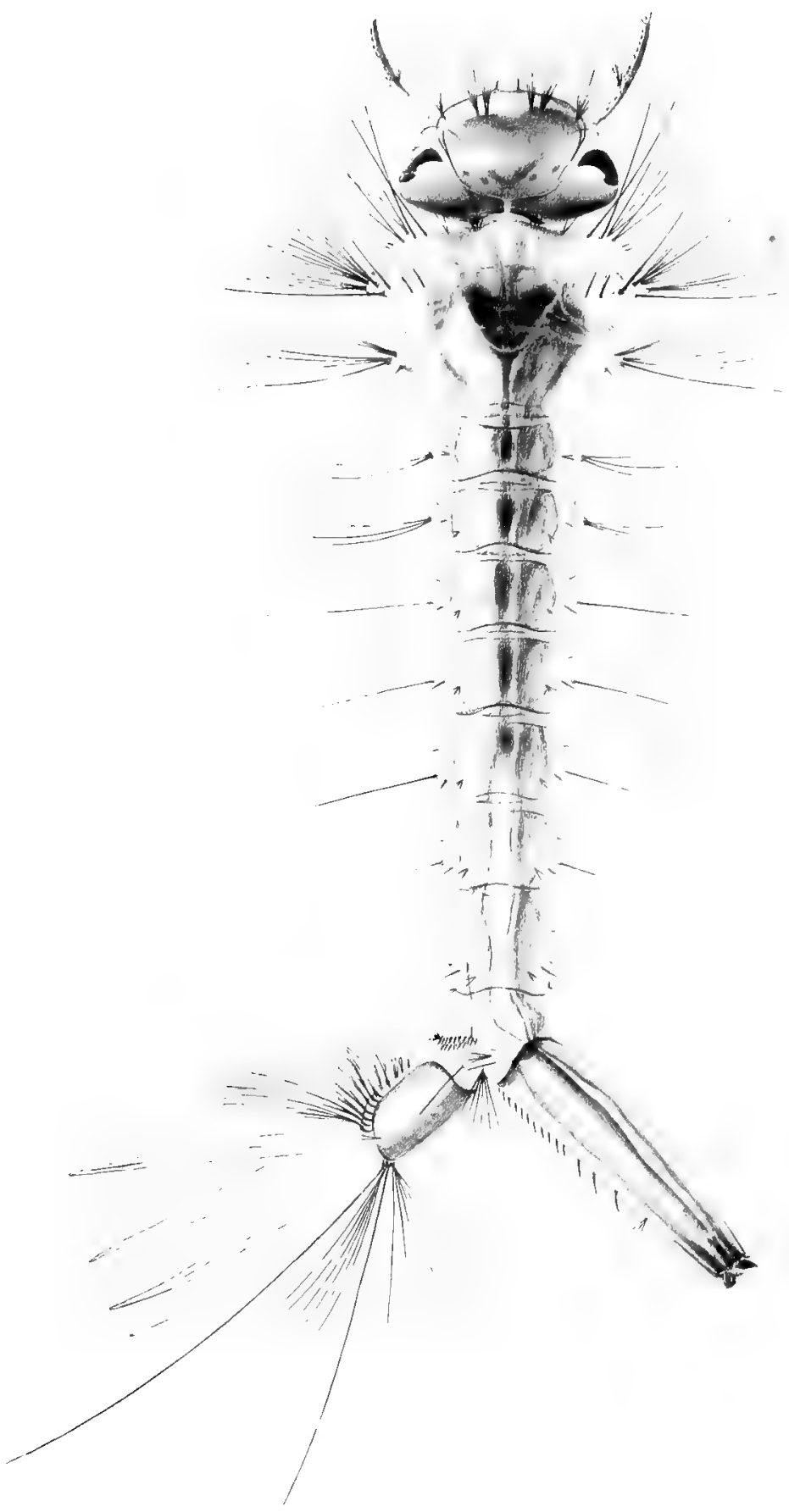

Larva: Aedes fuscus Osten Sacken.

oneate, fin. 



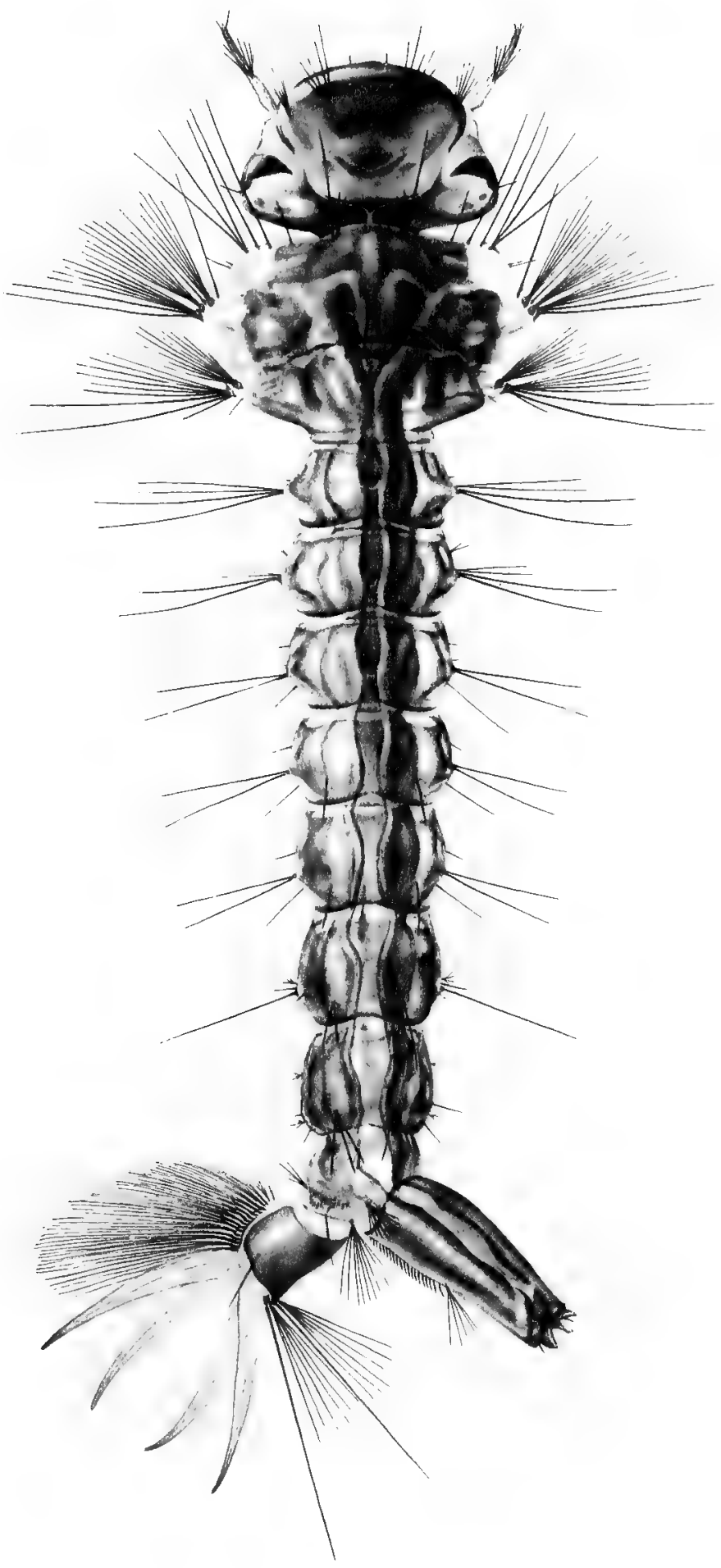

Larva: Aedes hirsuteron Theobald. 



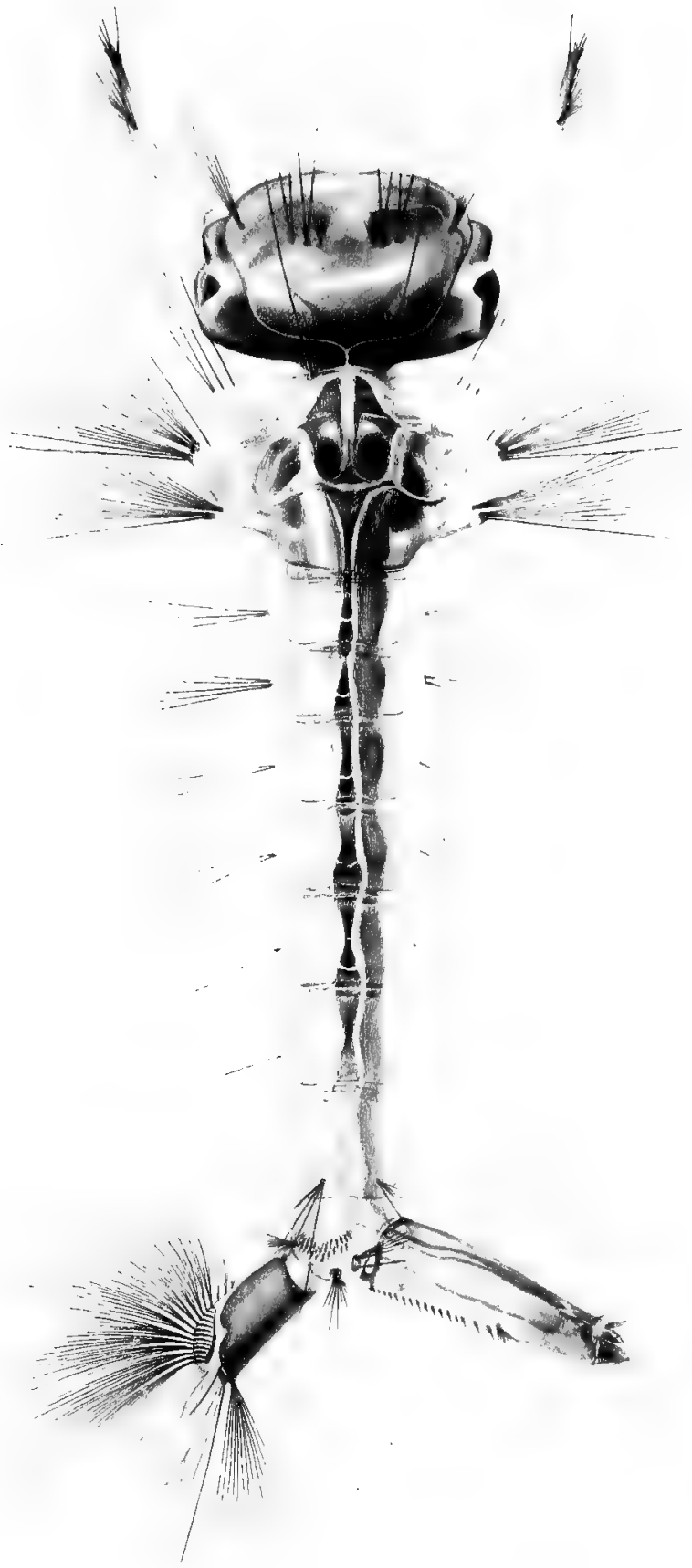

Larva: Aedes aurufer Coquilletl. 



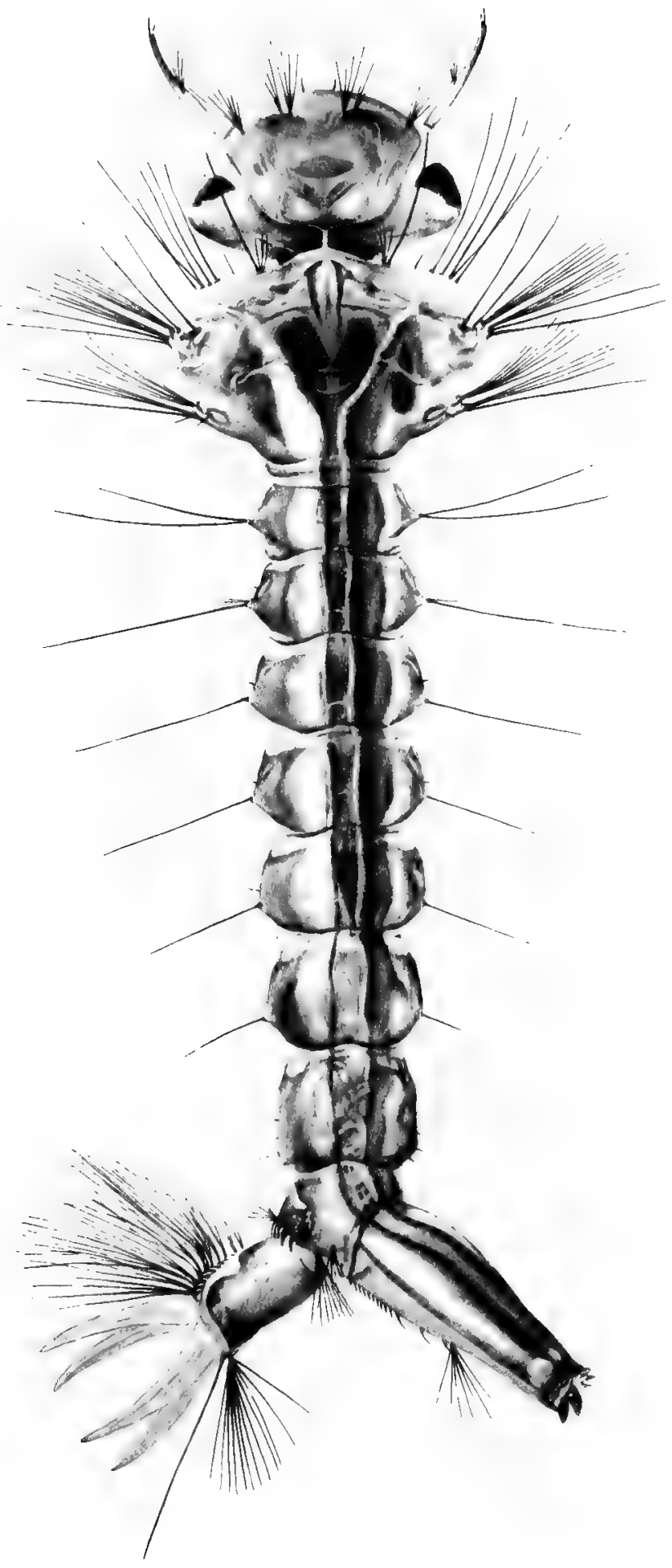

Larva: Aedes imusiger lJalker. 



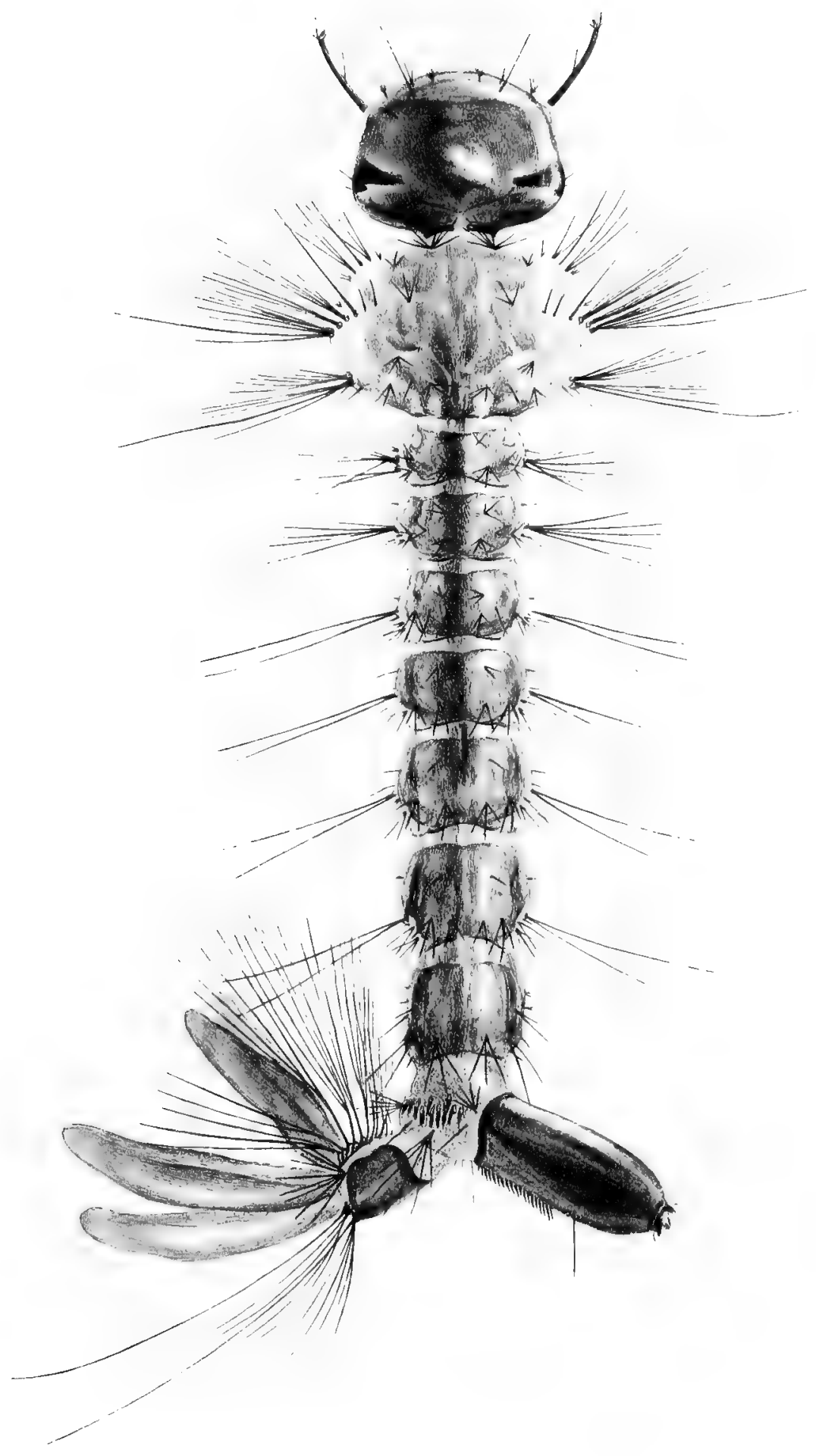

Larva: Aedes triseriatus Say. 



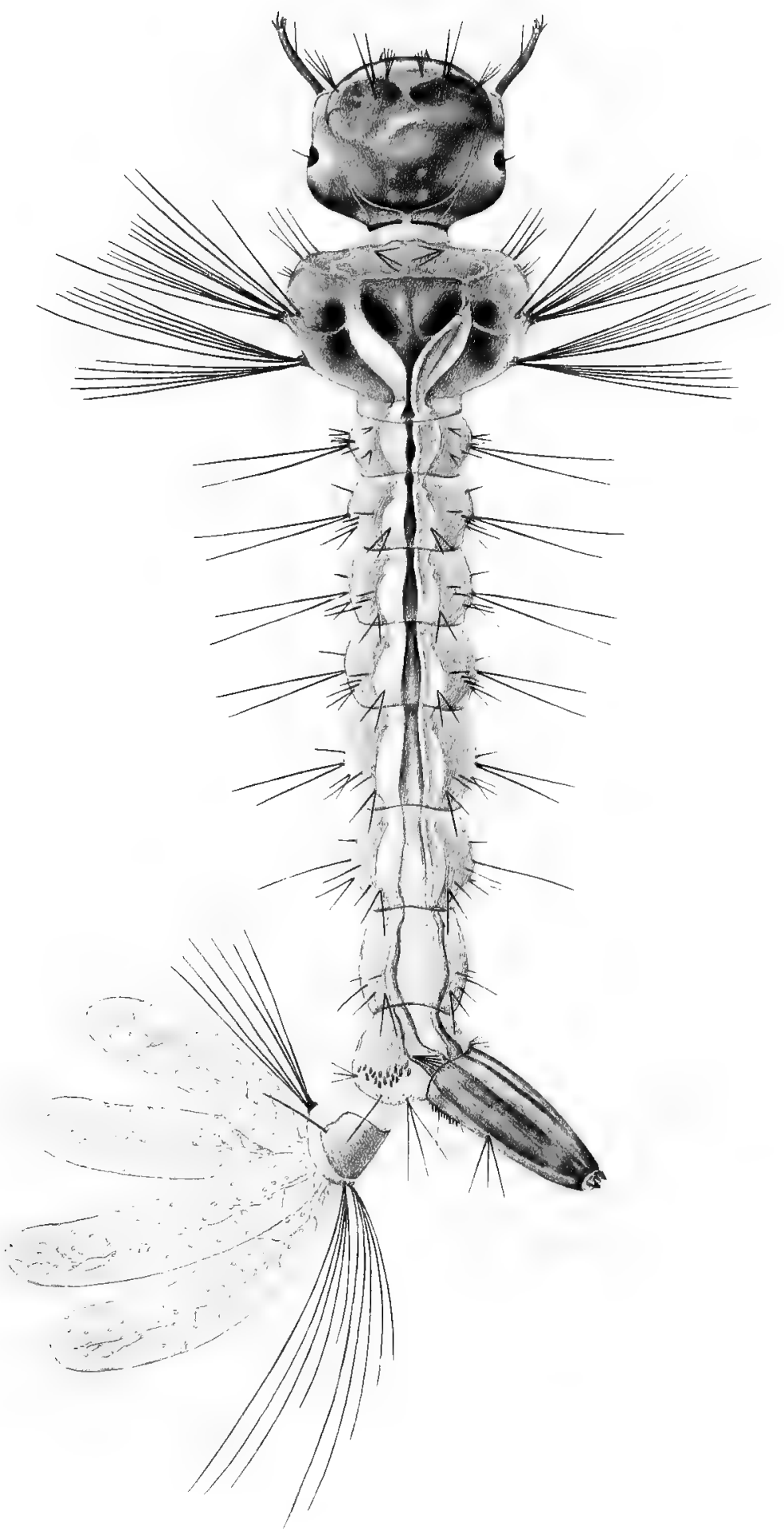

Larva: Aedes varipalpus Coqullett 



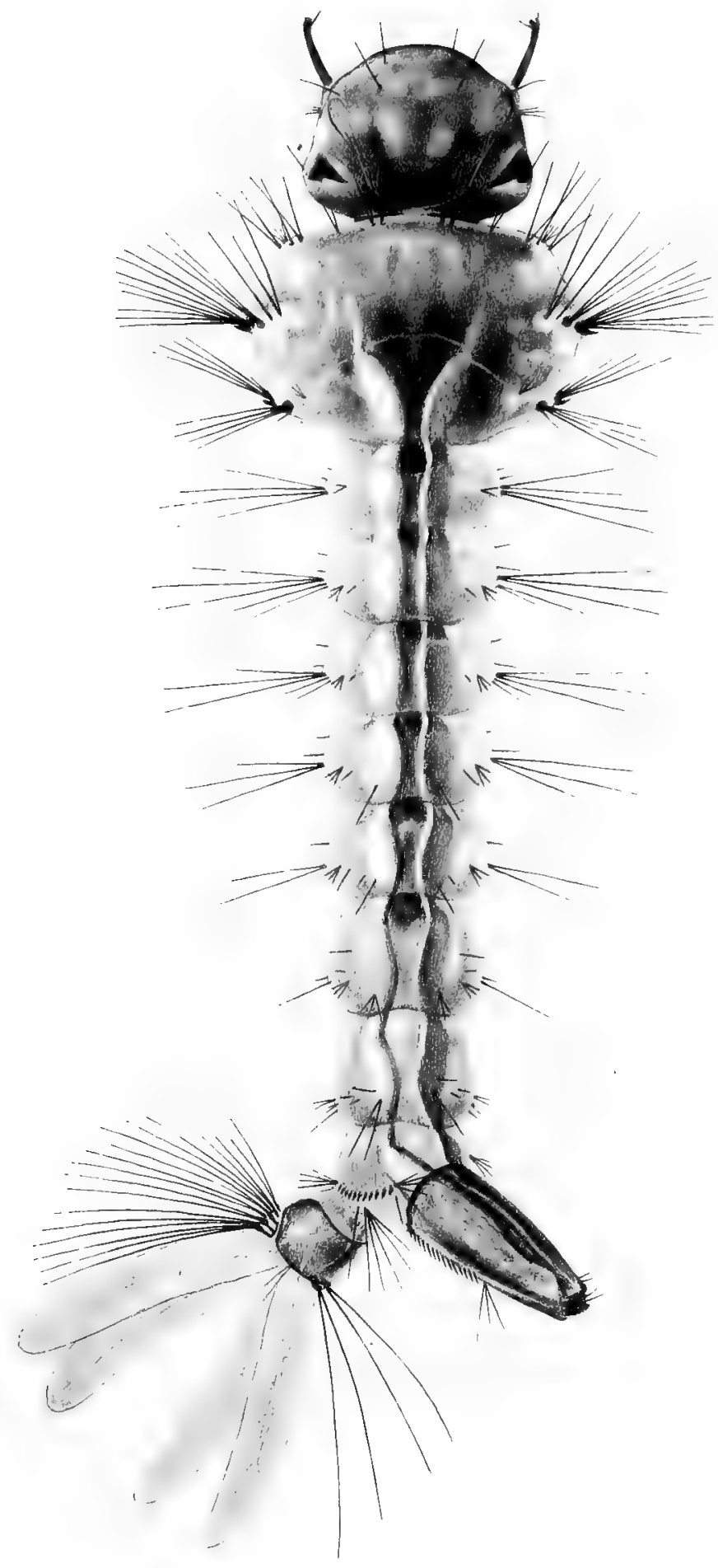

Larva: Aedes calopus Meigen. 



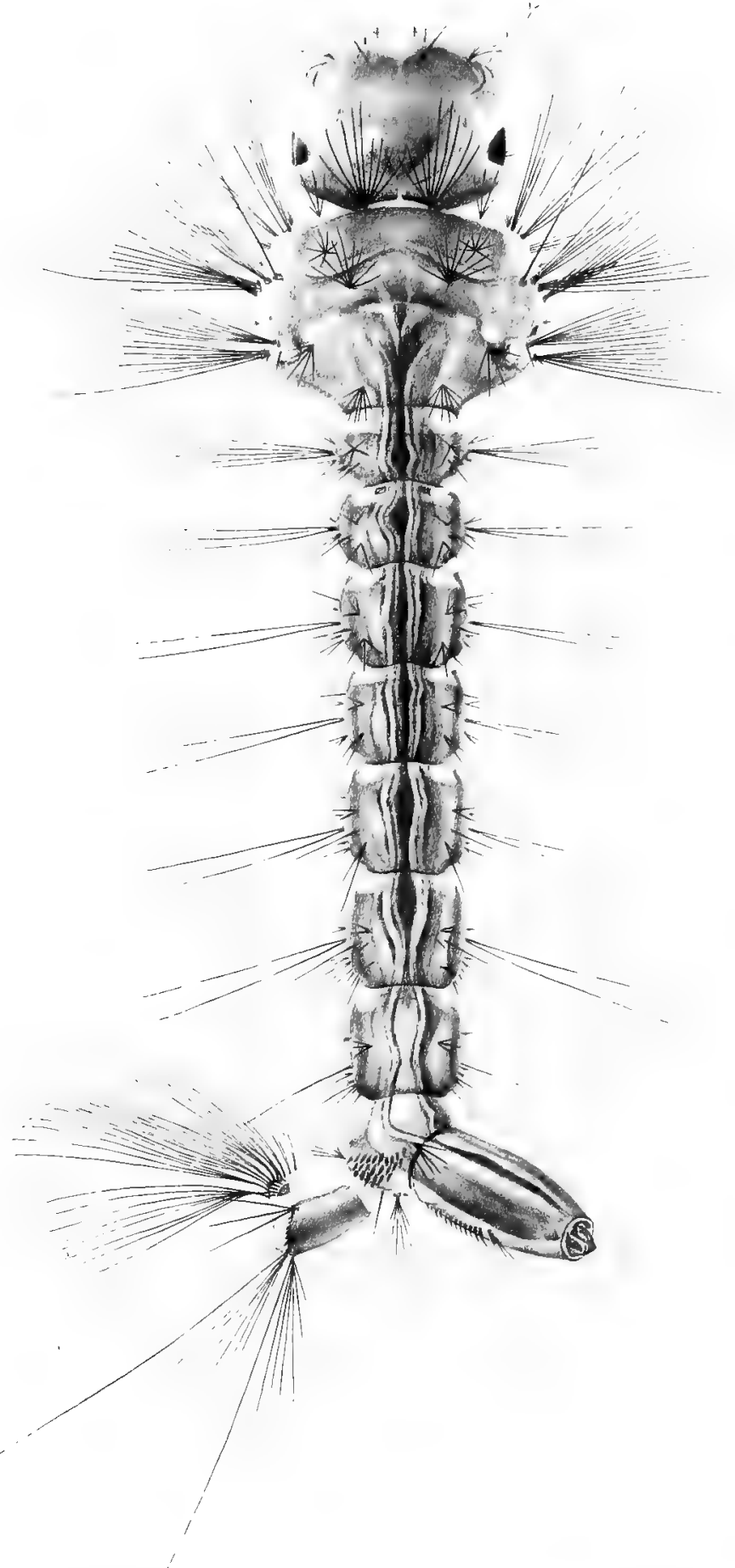

Larva: Stegoconops lucifer Howard, Dyar \& Knab. 



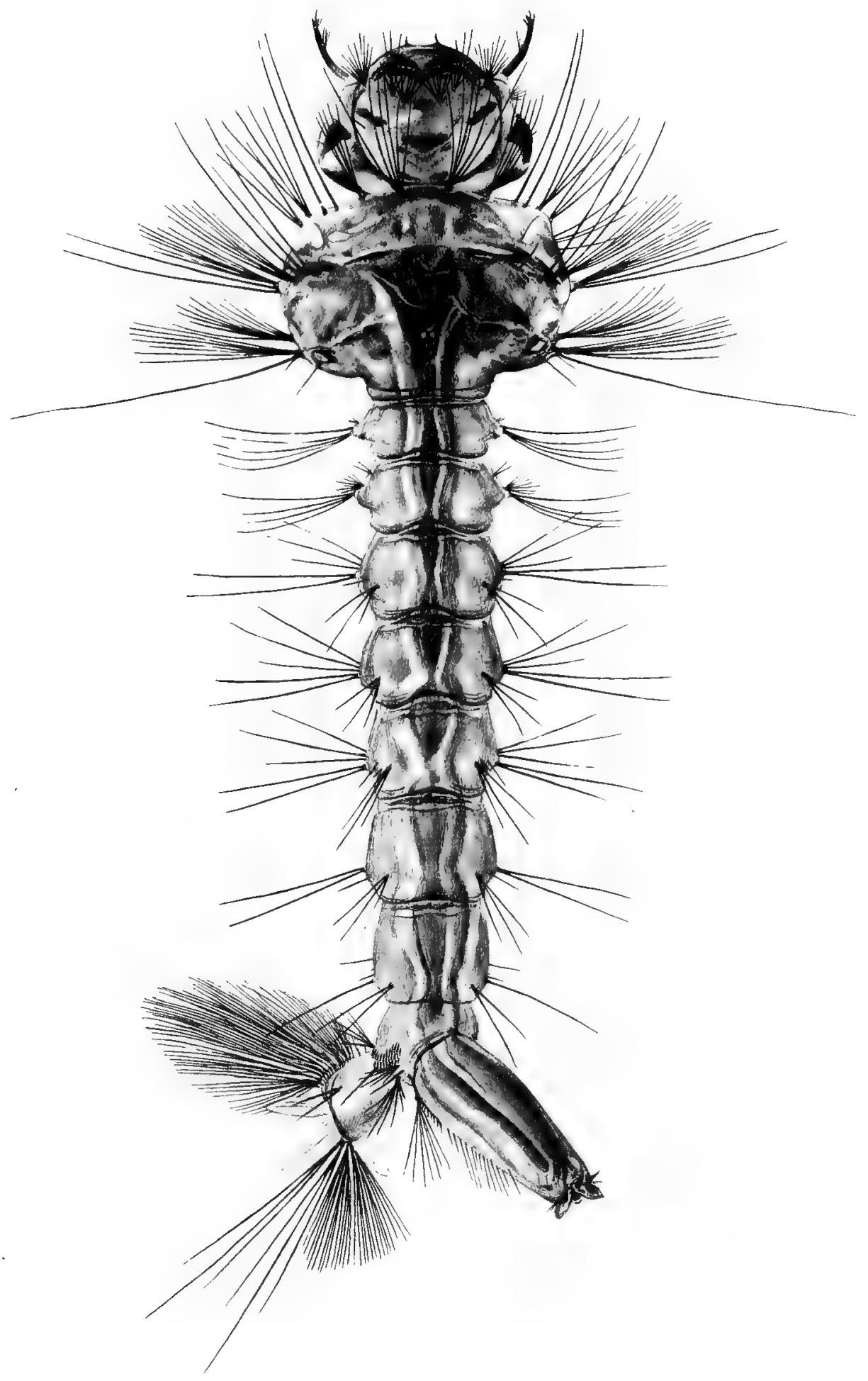

Larva: Culiseta imoruatus Williston. 



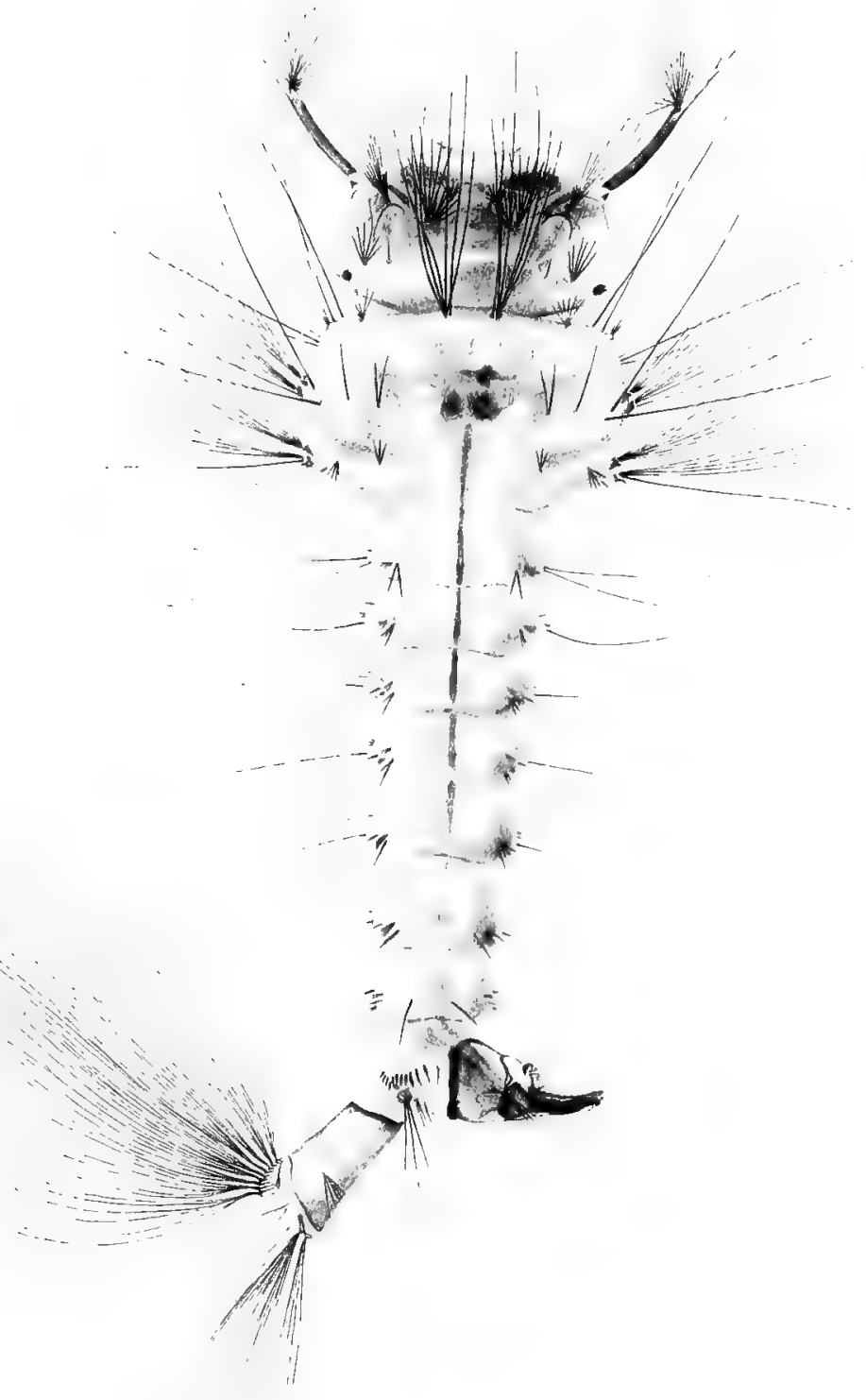





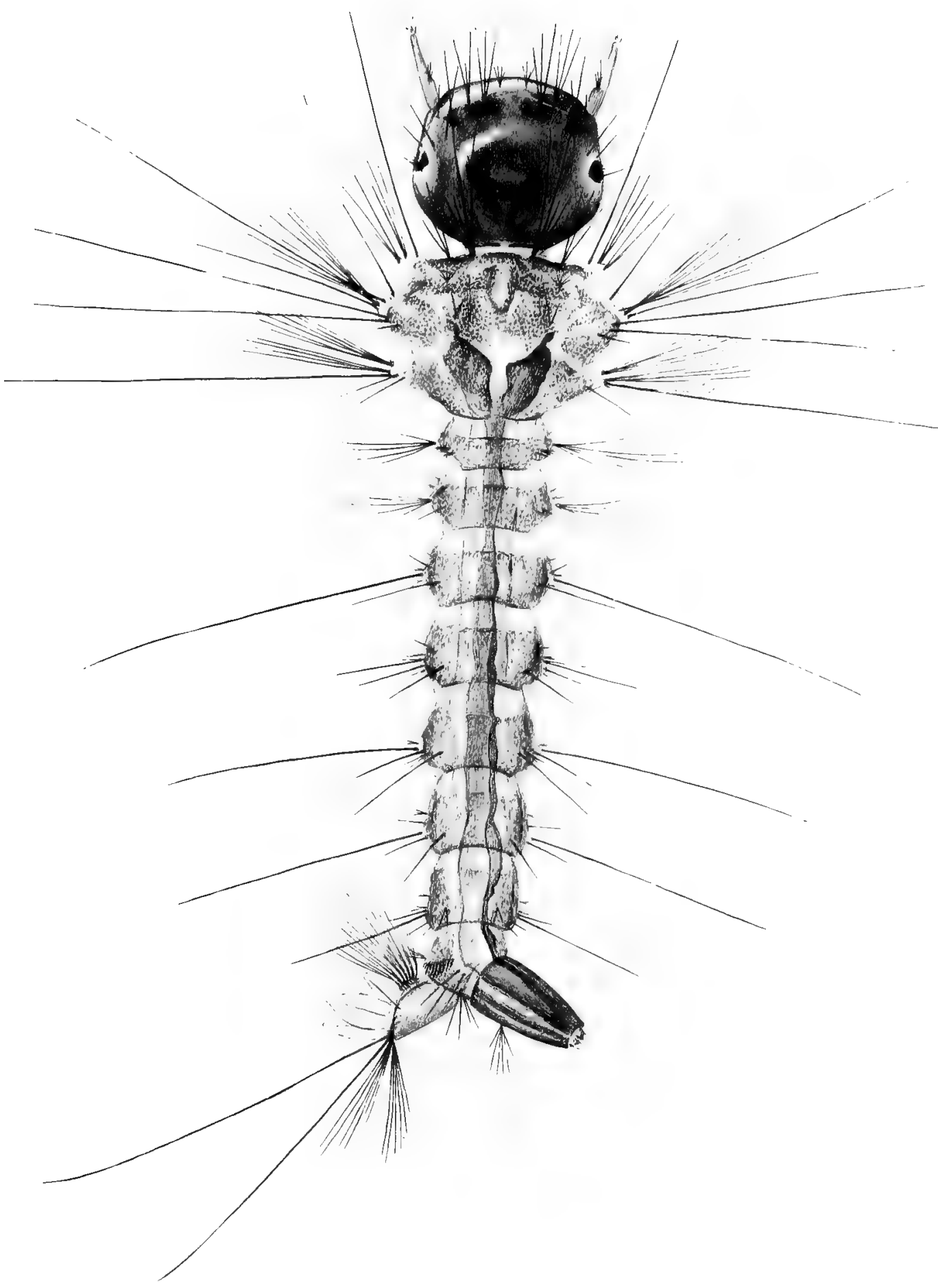

Larva: Bancroftia slgnifer Coqullelt, stage III. 



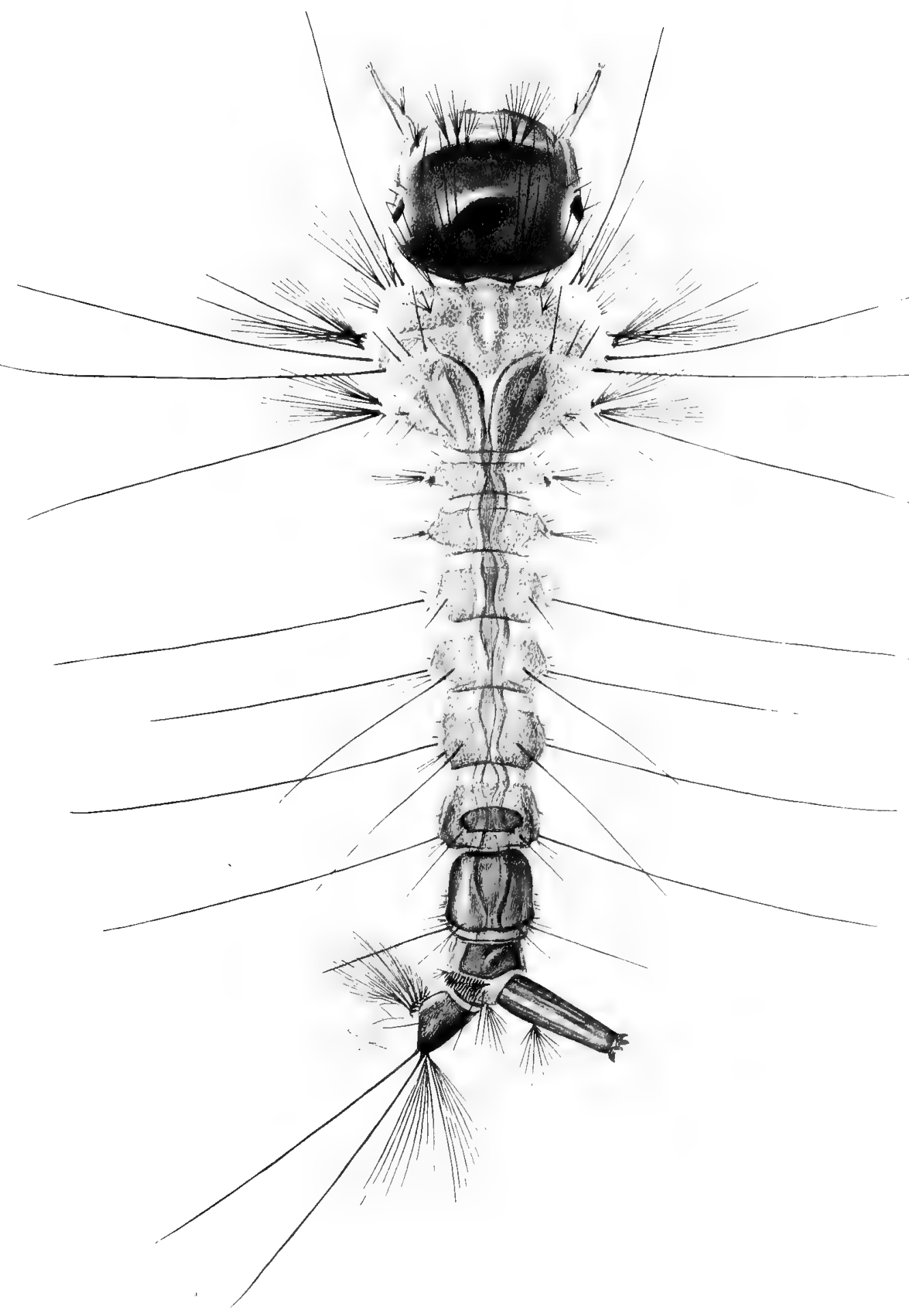

Larva: Bancroftia signifor Coqullctt. 



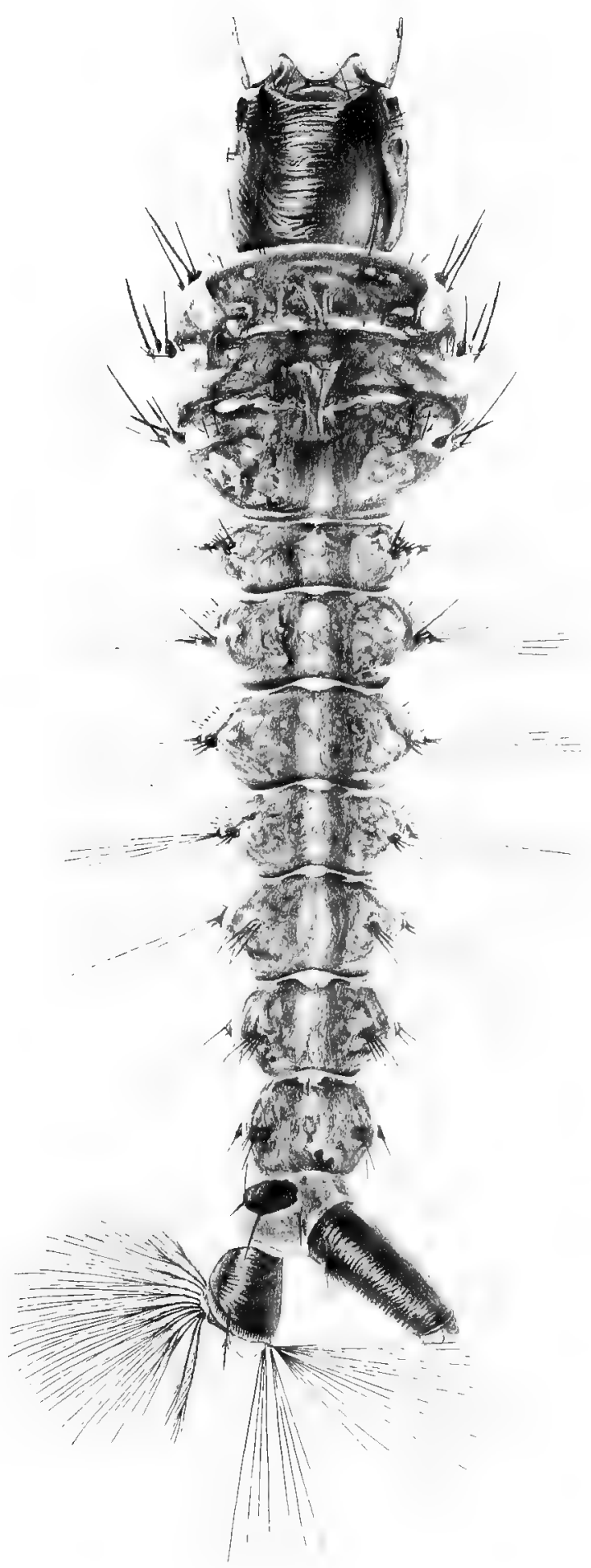

Larva: Megarhinus septentrionalis Dyar \& Knab. 



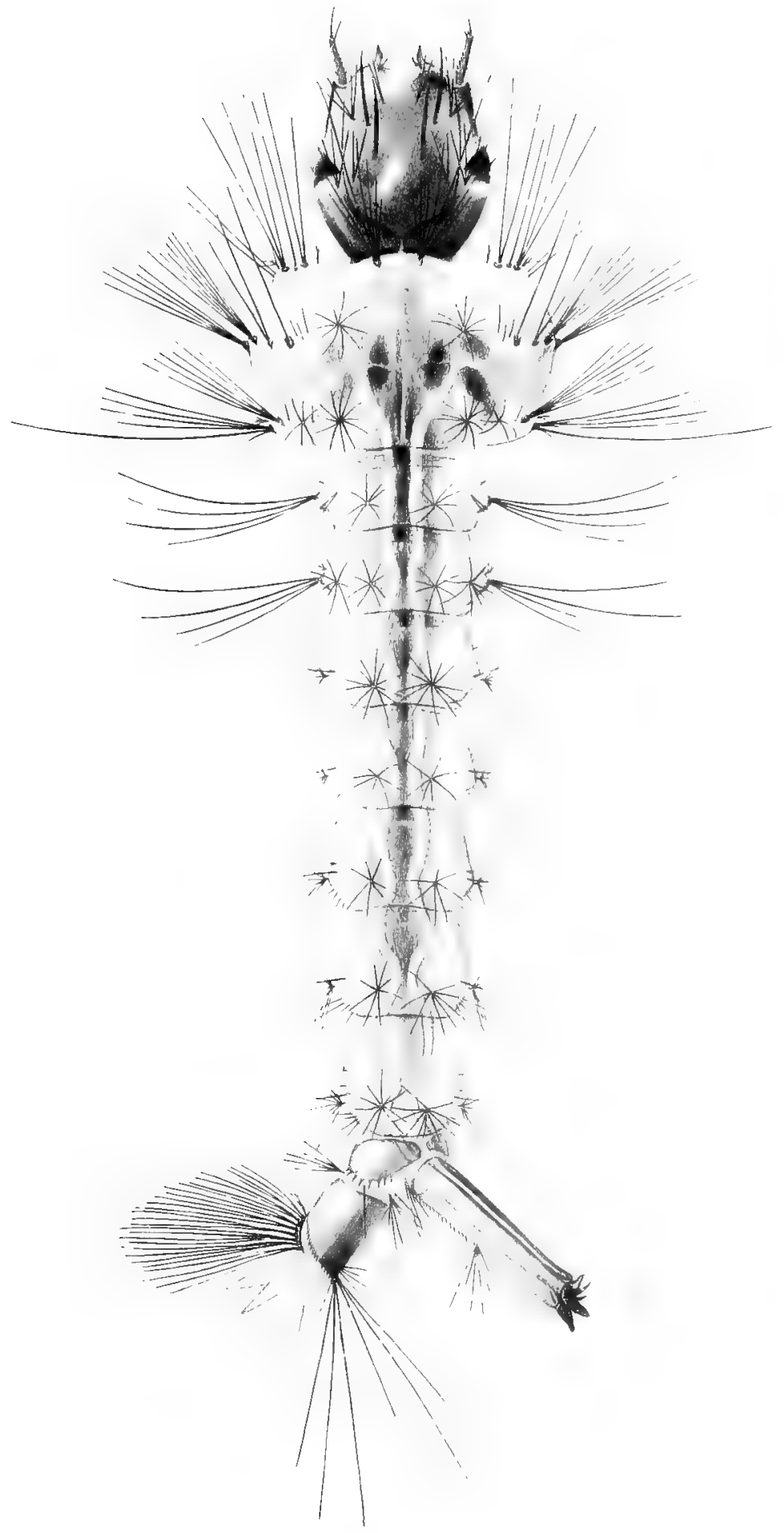

Larva: Uranotænia sapphirinus Osten Sacken. 



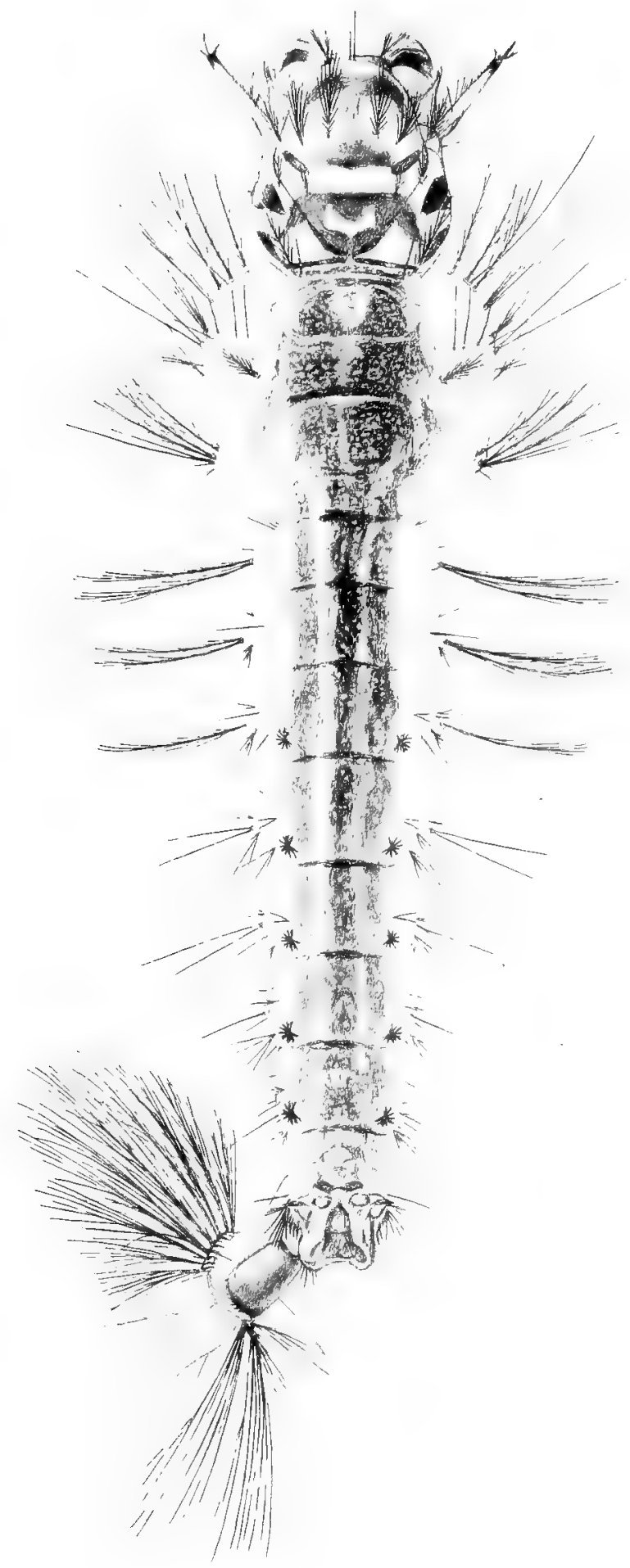

Larva: Anopheles punctipennis Say. 



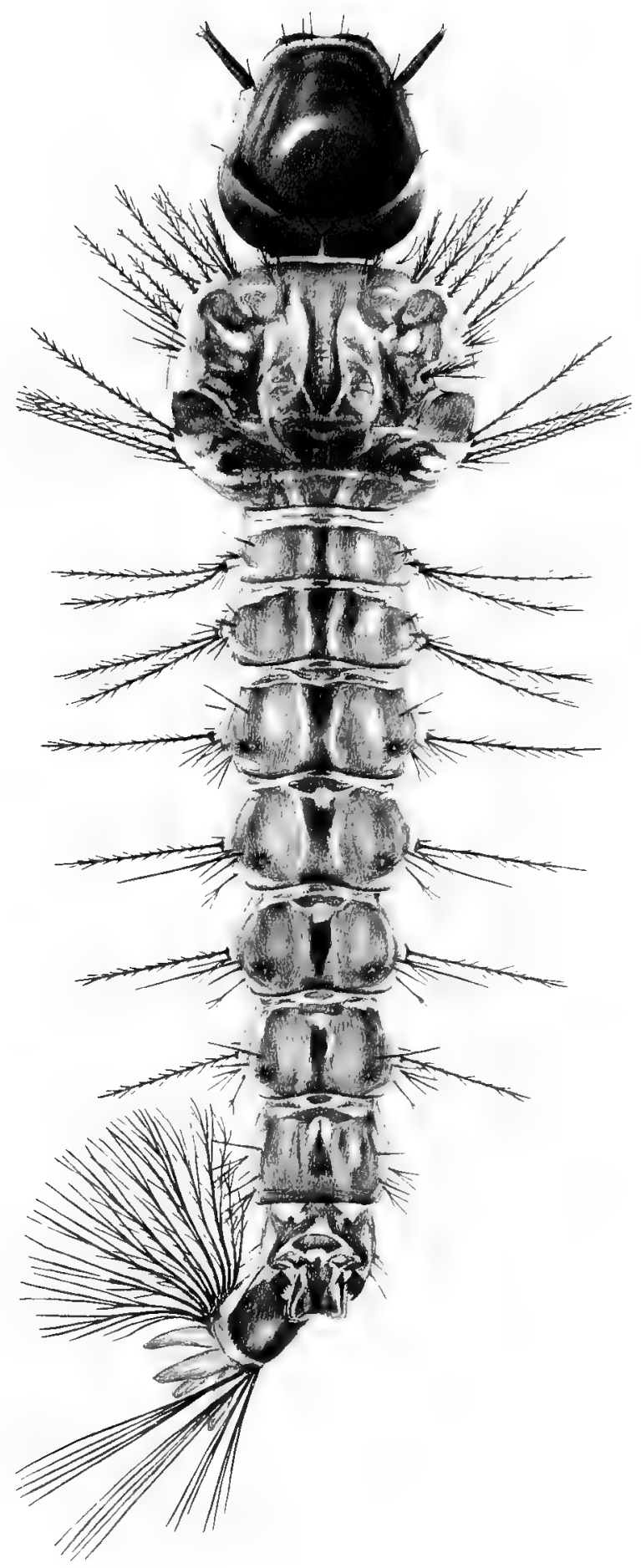

Larva: Cœlodiazesis barberi Coquillett. 


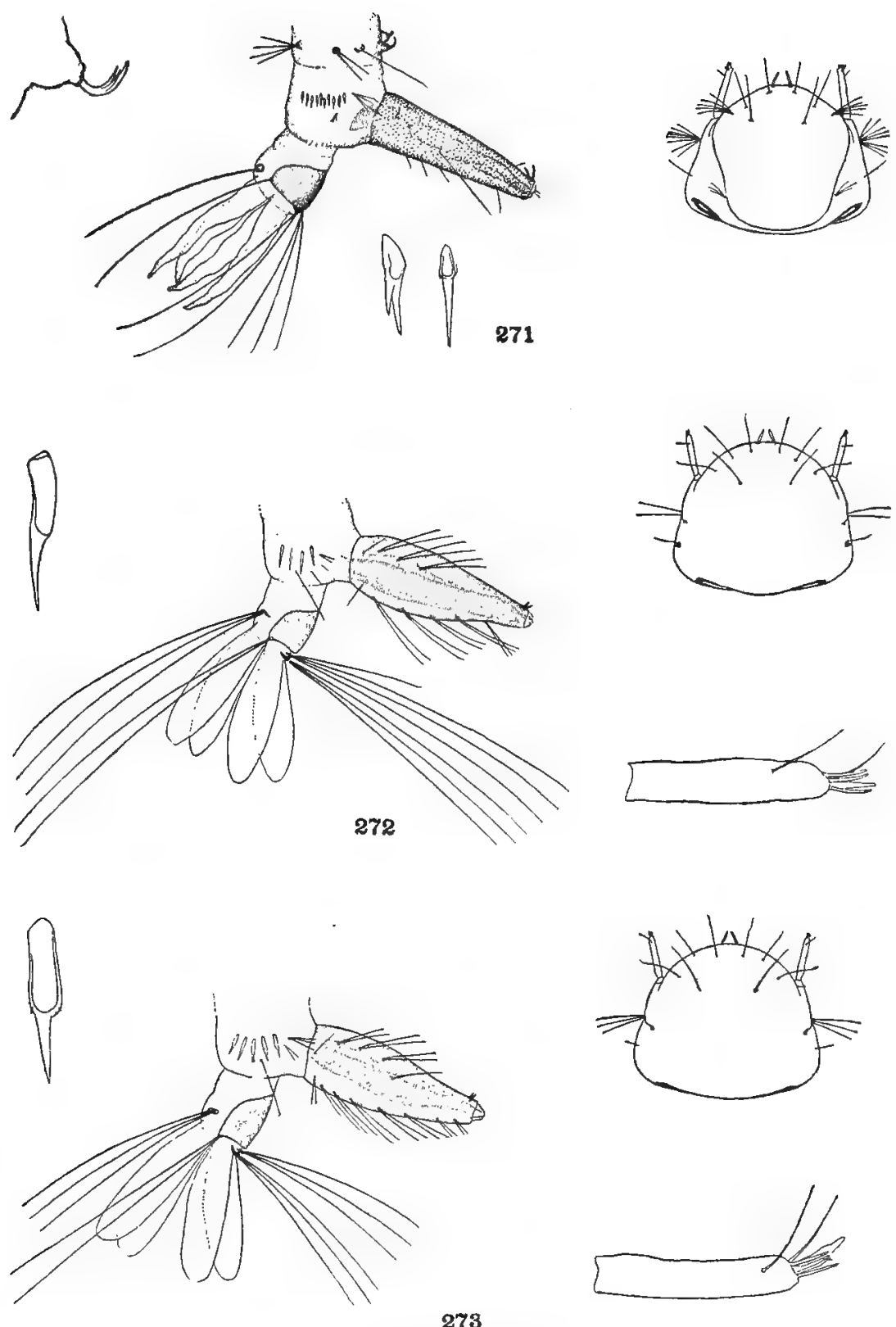

DETAILS OF LARVAE.

27I. Sabethinus identicus Dyar \& Knab.

272. Limatus durhamii Theobald.

273. Limatus cacophrades Dyar \& Knab. 


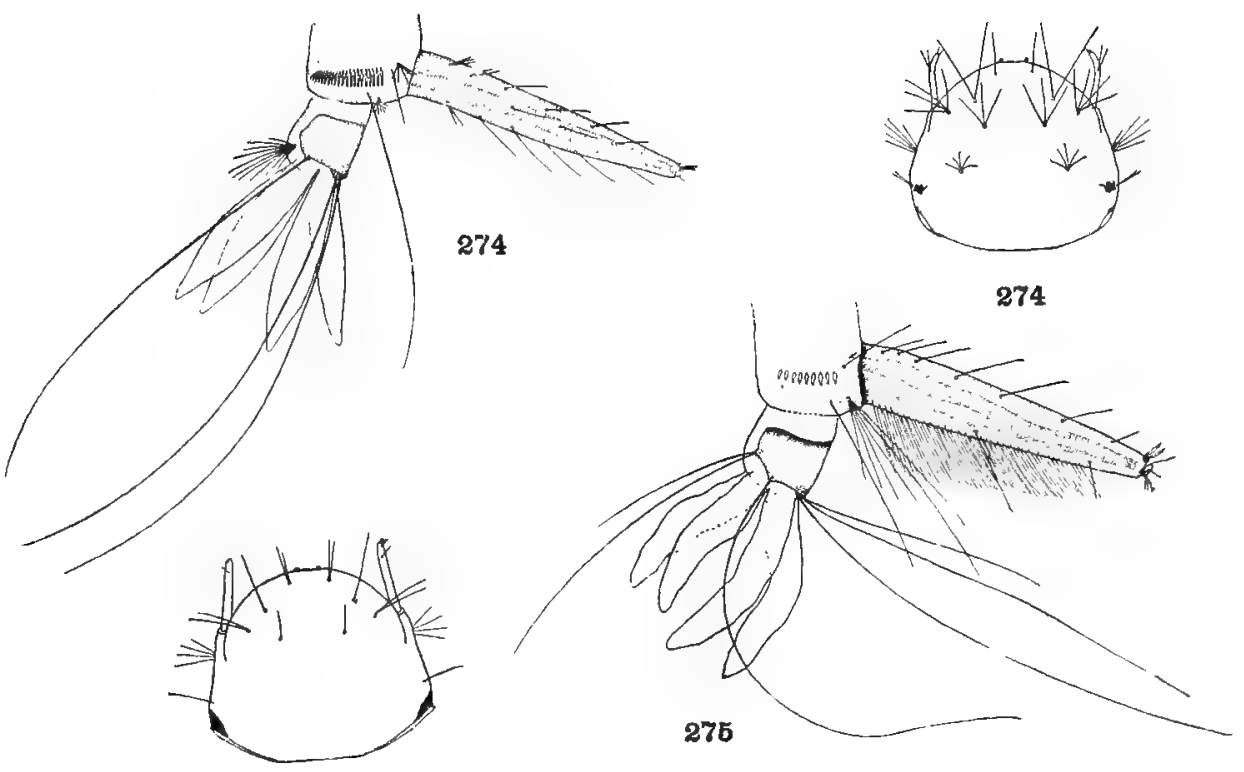

276
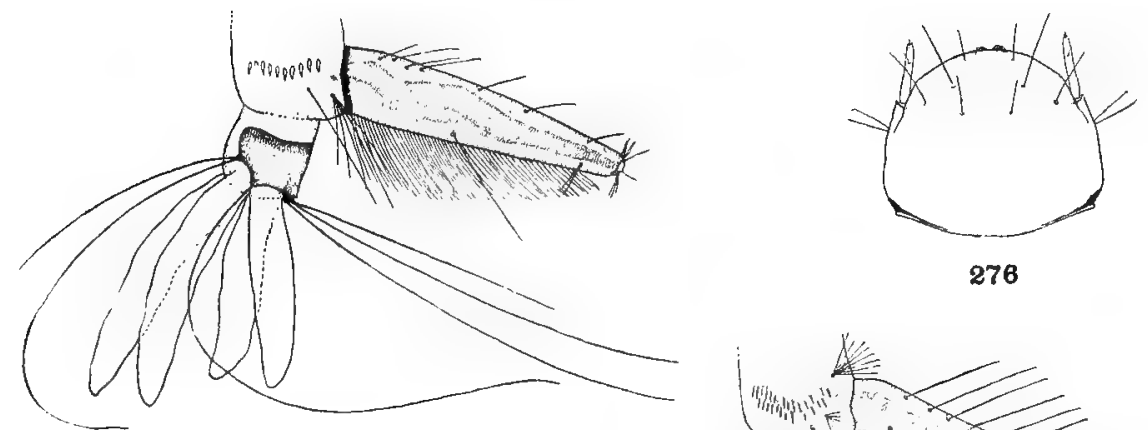

276

276
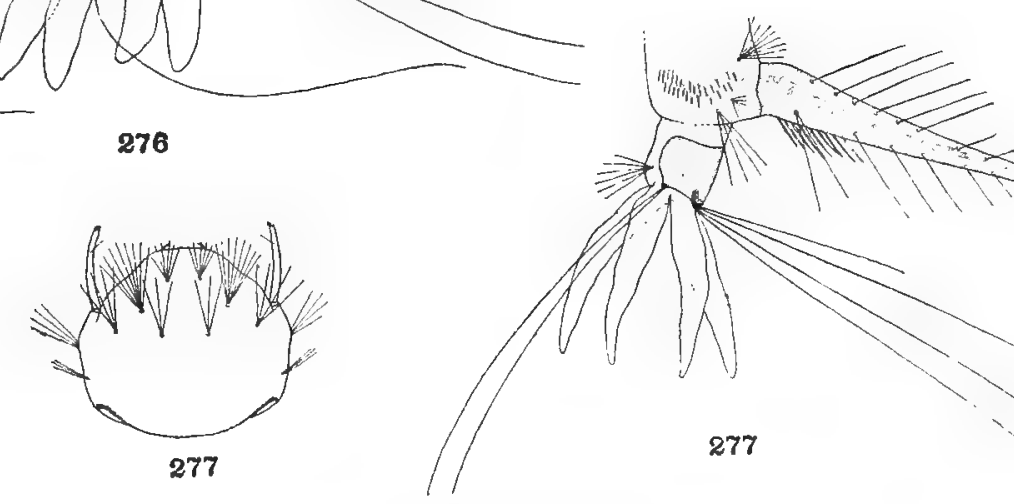

DETAILS OF LARVE.

274. Wyeomyia philophone Dyar \& Knab.

275. Wyeomyia pandora $D y a r$ \& $K n a b$.

276. Wyeomyia canfieldi Dyar \& $K n a b$.

277. Wyeomyia trinidadensis Theobald. 



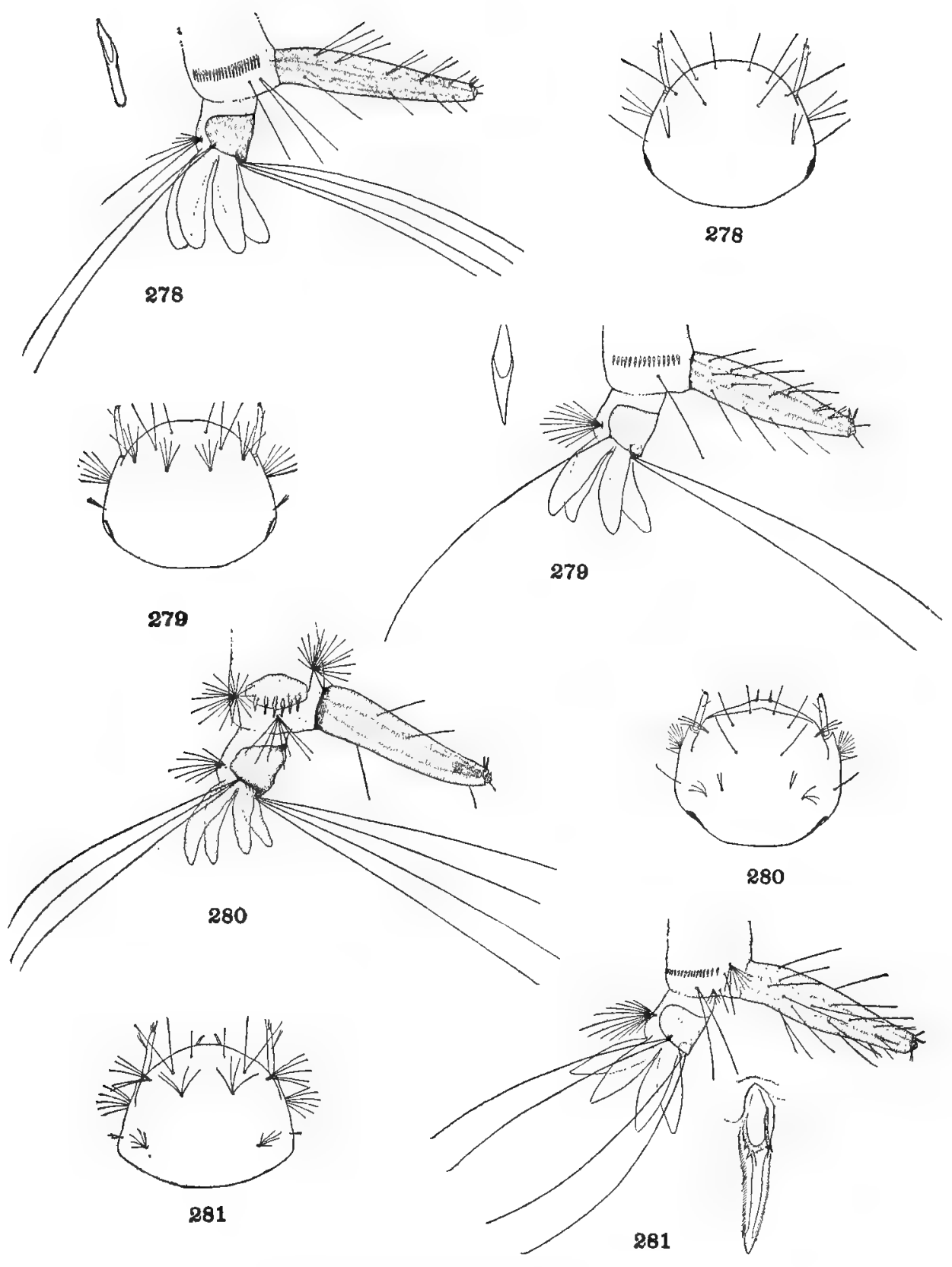

DETAILS OF LARV $Æ$.

278. Wyeomyia sororcula Dyar \& $K n a b$.

279. Wyeomyia mitchellii Theobald.

280. Wyeomyia codiocampa Dyar \& $K n a b$.

281. Wyeomyia ablabes Dyar \& $K n a b$. 



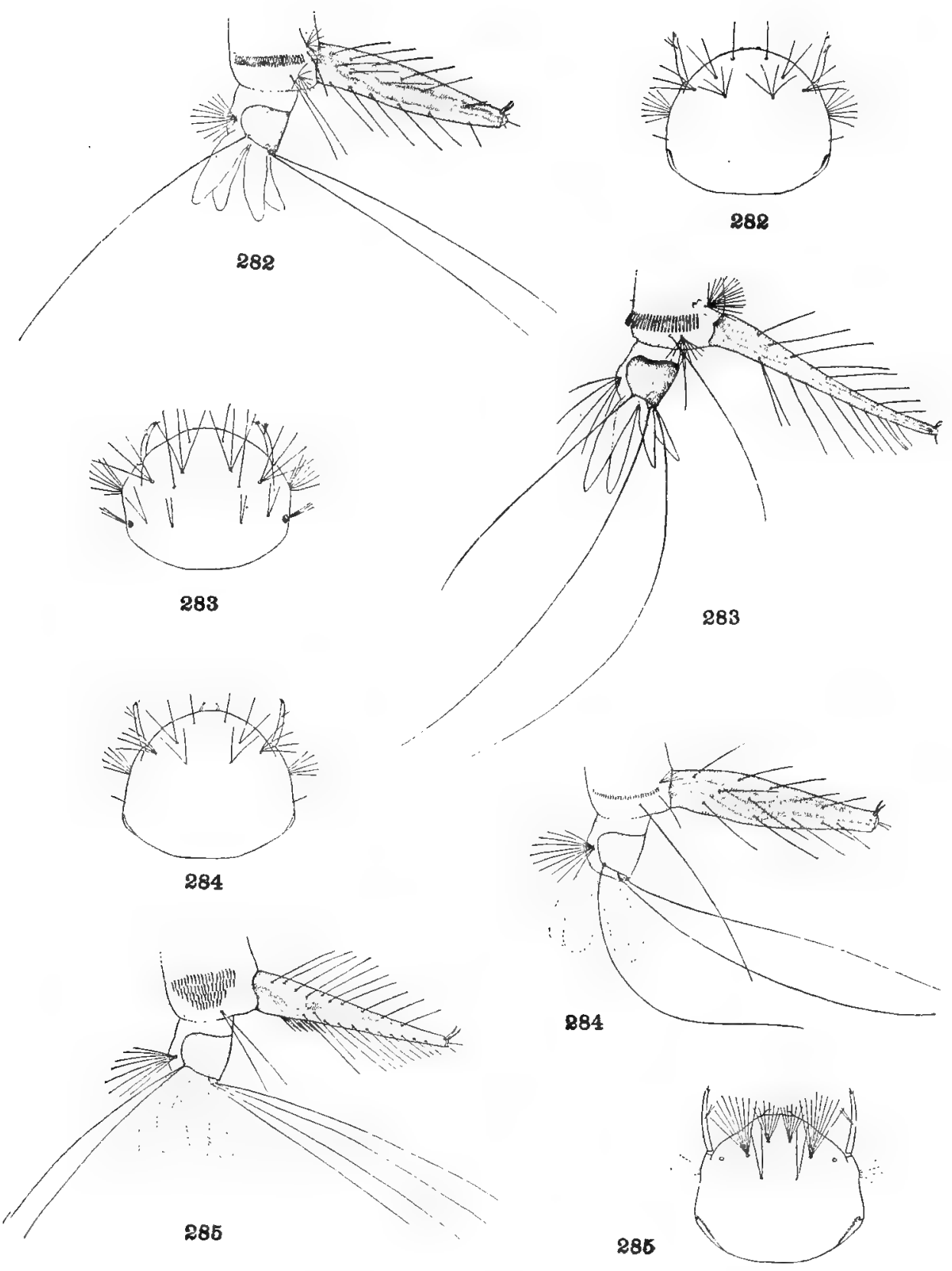

DETAILS OF LARV平.

282. Wyeomyia labesba Dyar \& Knab.

283. Wyeomyia abebela Dyar \& Knab.

284. Wyeomyia abia Dyar \& Knab.

285. Wyeomyia autocratica Dyar \& $K n a b$. 


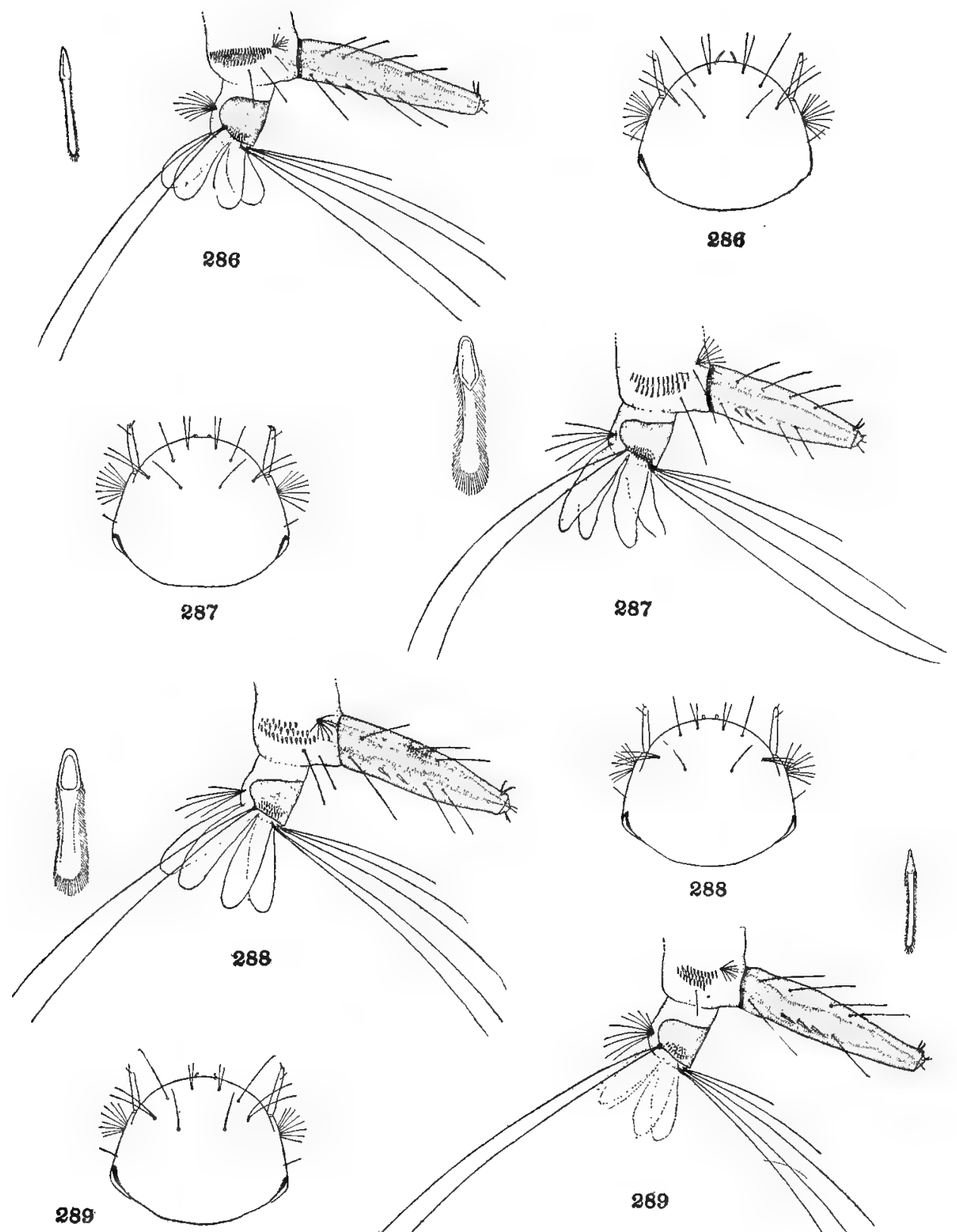

289

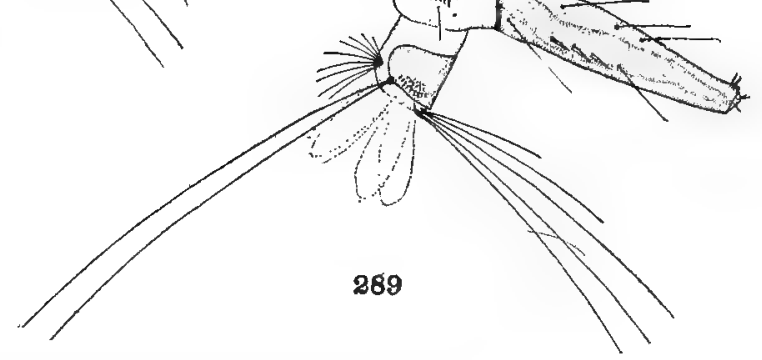

DETAILS OF LARVAE.

286. Wyeomyia pseudopecten Dyar \& Knab.

287. Wyeomyia eloisa Howard, Dyar \& Knab.

288. Wyeomyia pantoia Dyar \& Knab.

289. Wyeomyia onidus Dyar \& Knab. 



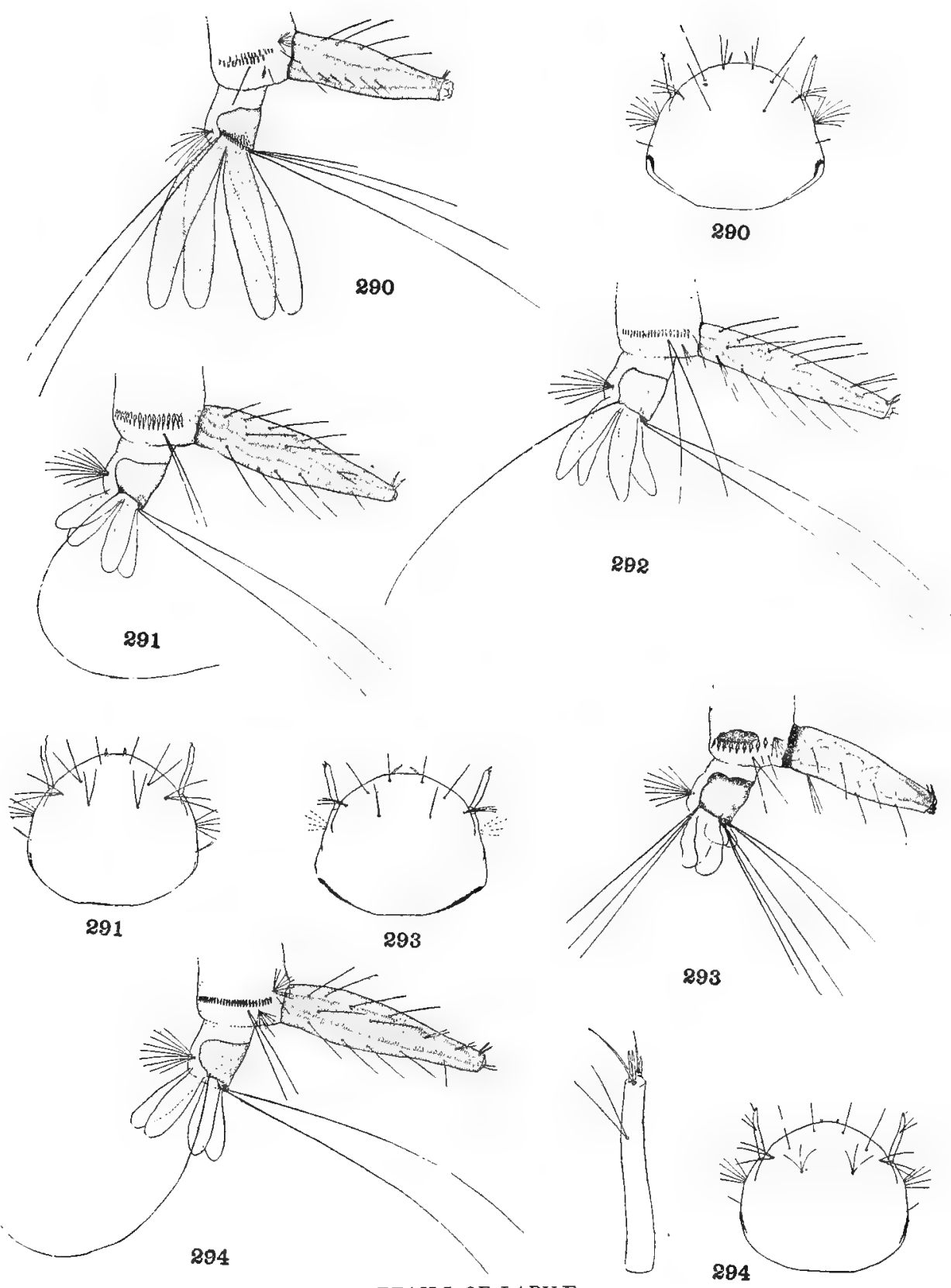

DETAILS OF LARYIE.

290. Wyeomyia cacodela Dyar \& Knab.

291. Wyeomyia chresta Dyar \& Knab.

292. Wyeomyia telestica Dyar \& Knab.

293. Wyeomyia bromeliarum Dyar \& Knab.

294. Wyeomyia abrachys Dyar \& Knab. 


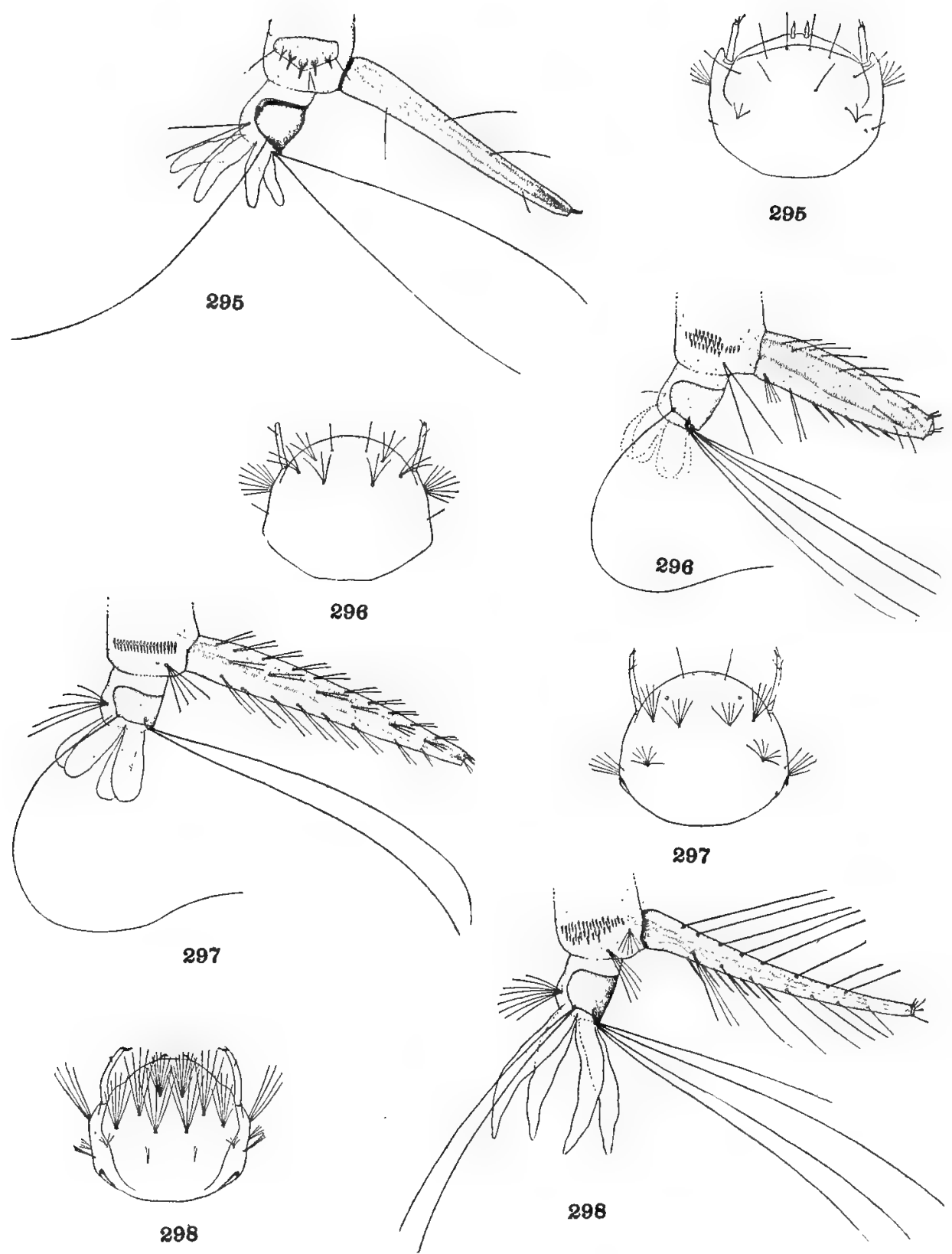

296

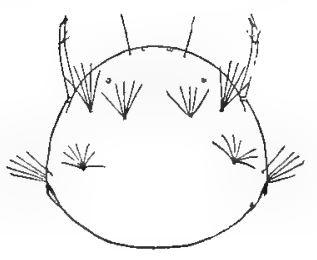

297
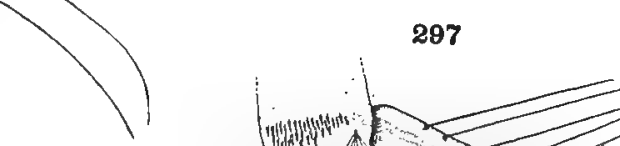


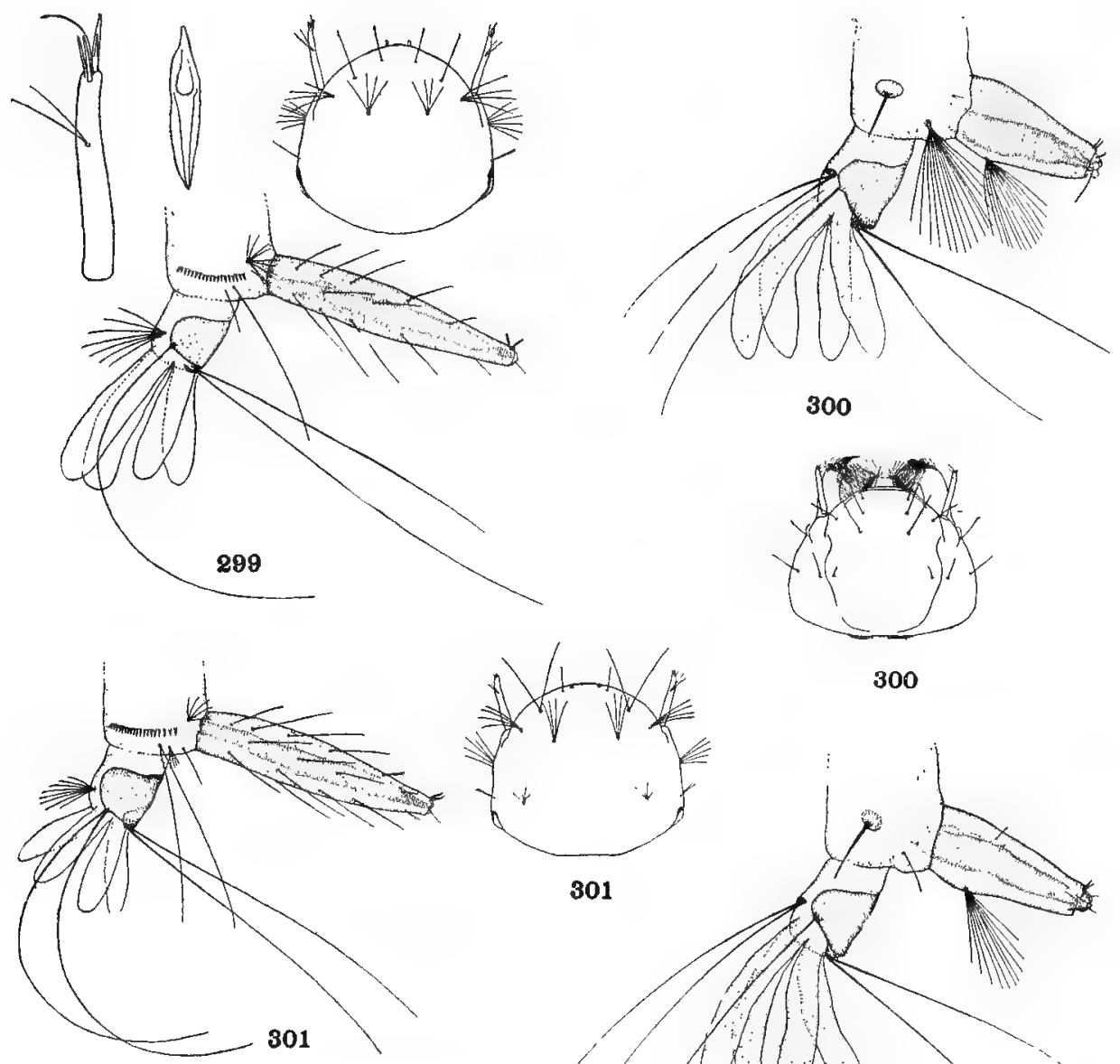

300
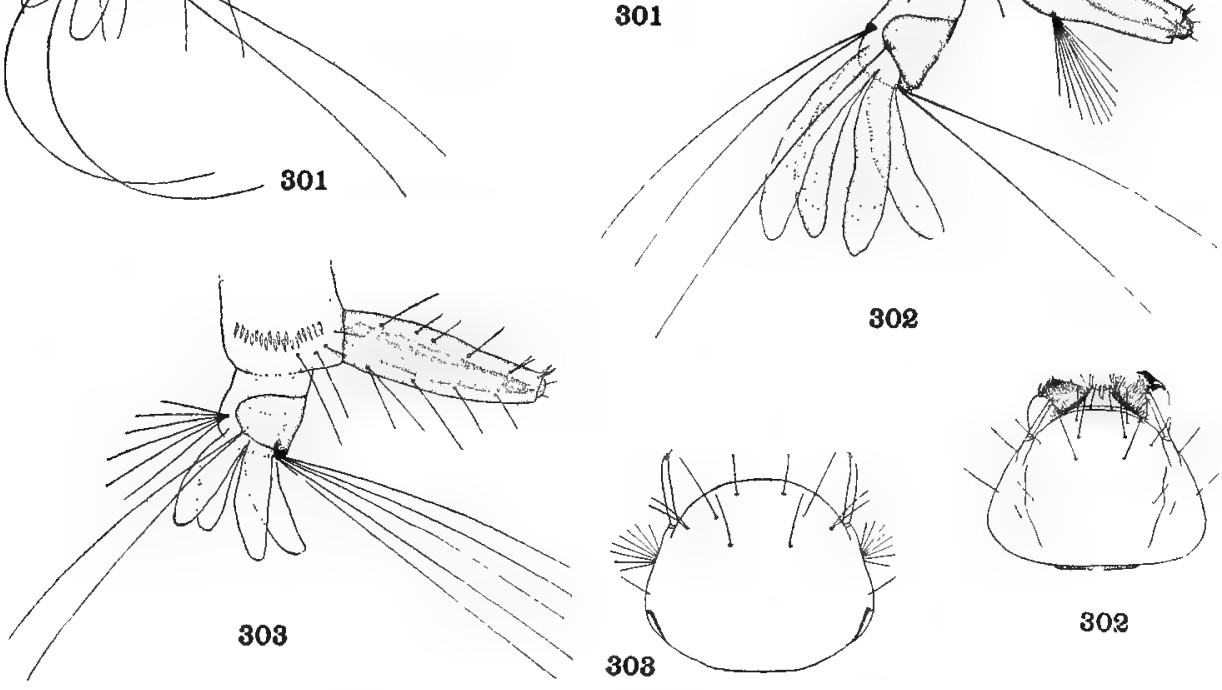

30.2

DETAILS OF LARVE.

299. Wyeomyia hapla Dyar \& Knab.

300. Joblotia trichorryes Dyar \& $K n a b$.

30I. Wyeomyia scotinomus Dyar \& $K n a b$.

302. Joblotia mogilasia Dyar \& Knab.

303. Wyeomyia hemisagnosta Dyar \& Knab. 



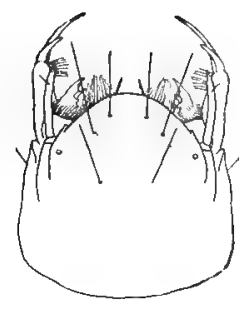

304
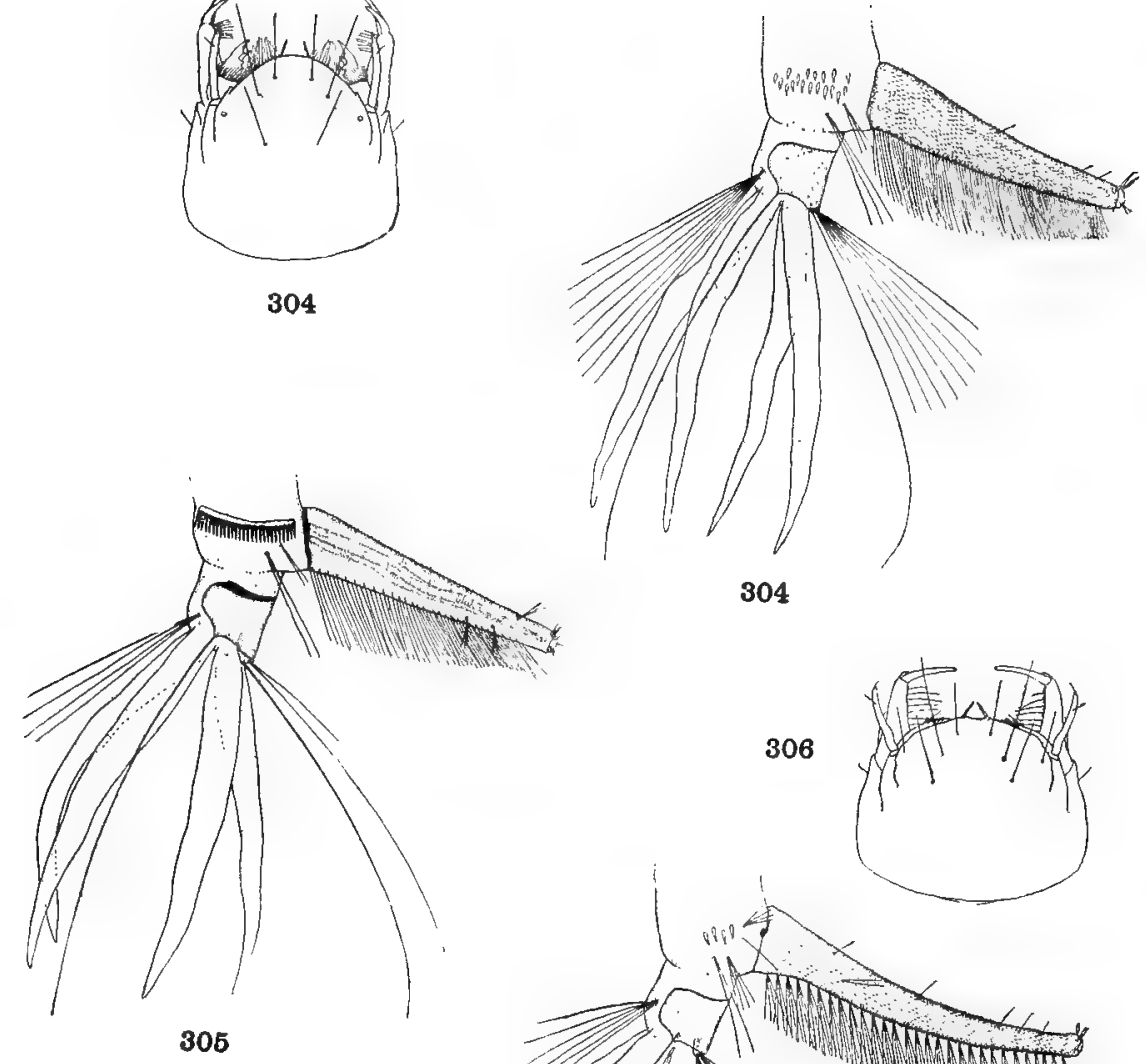

304
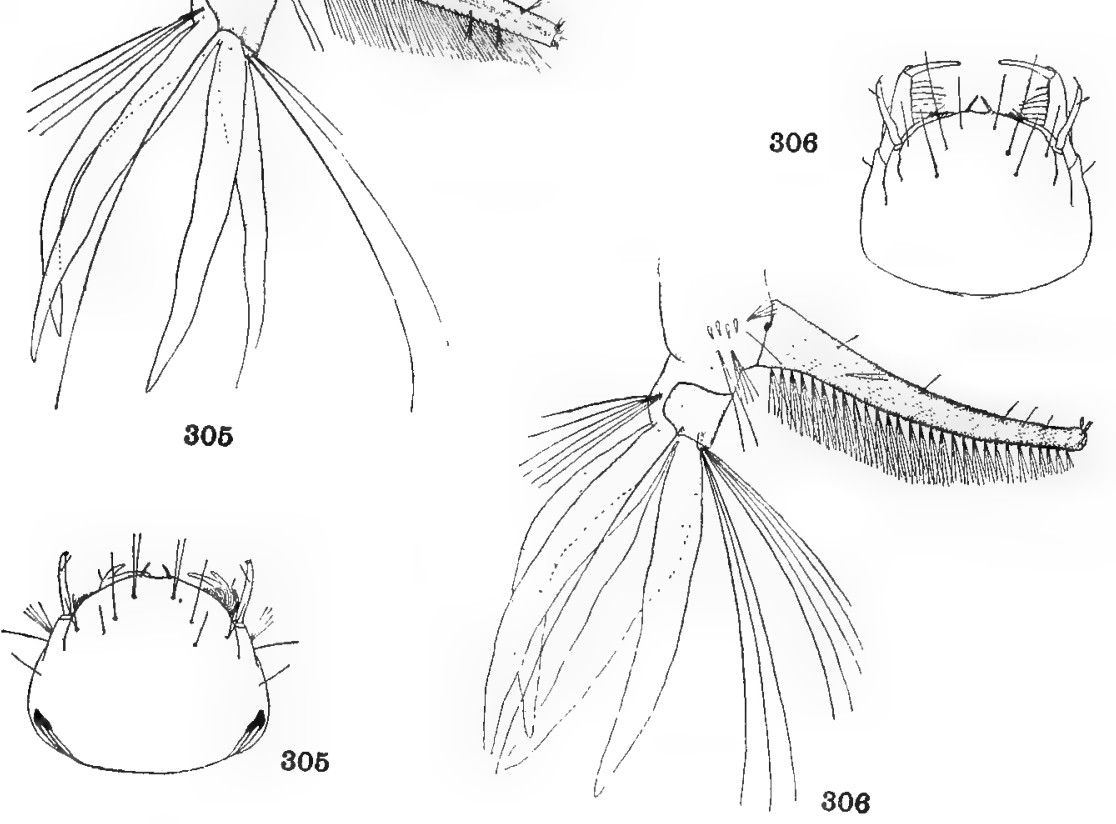

DETAILS OF LARVI.

304. Lesticocampa rapax Dyar \& Knab.

305. Lesticocampa dicellaphora Howard, Dyar \& Knab.

306. Lesticocampa culicivora Dyar \& $K n a b$. 


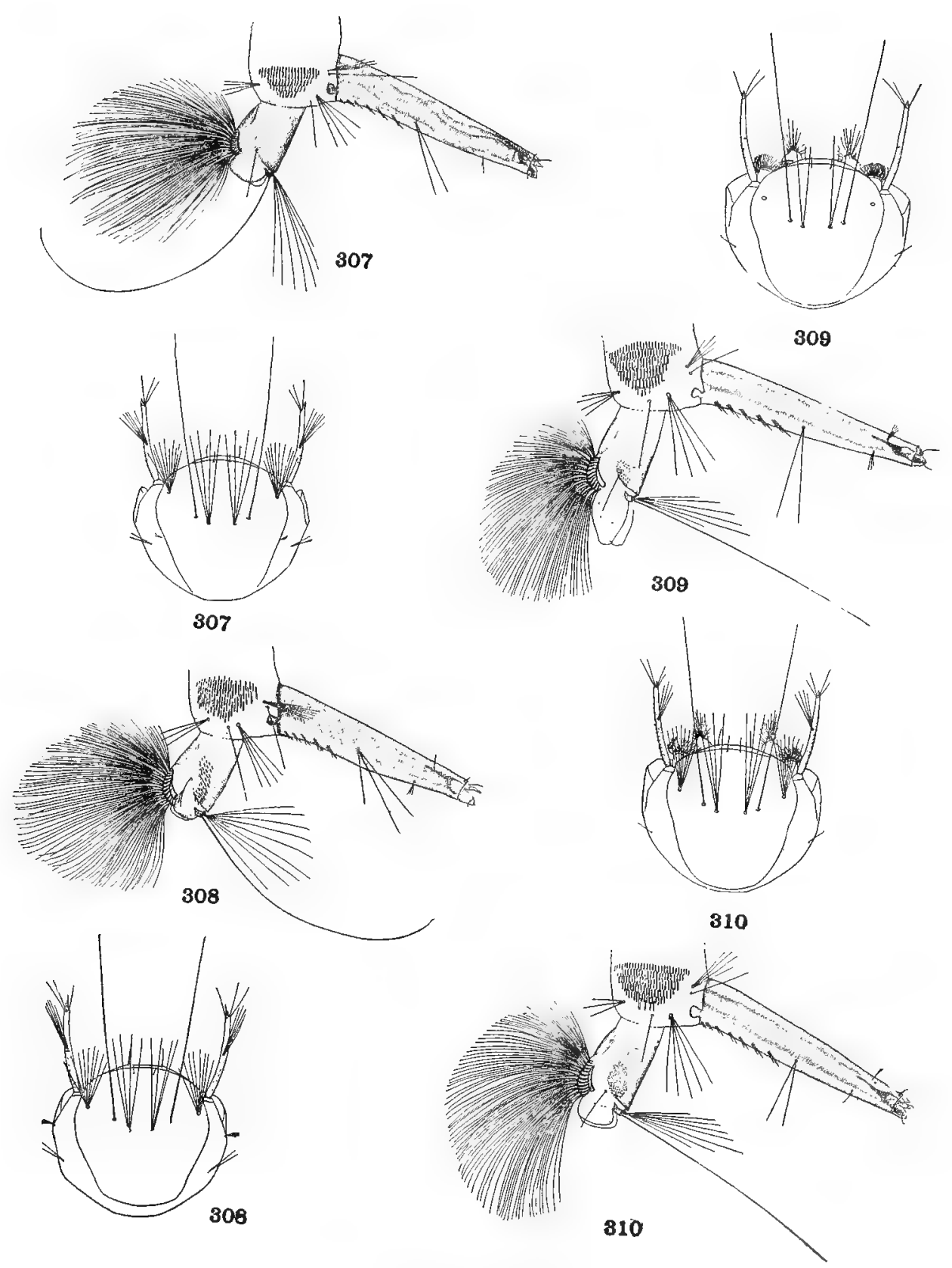

DETAILS OF LARVE.

307. Deinocerites melanophylum Dyar \& $K n a b$.

308. Deinocerites pseudes Dyar \& Knab.

309. Dinomimetes epitedeus $K n a b$.

310. Deinocerites troglodytus Dyar \& $K n a b$. 



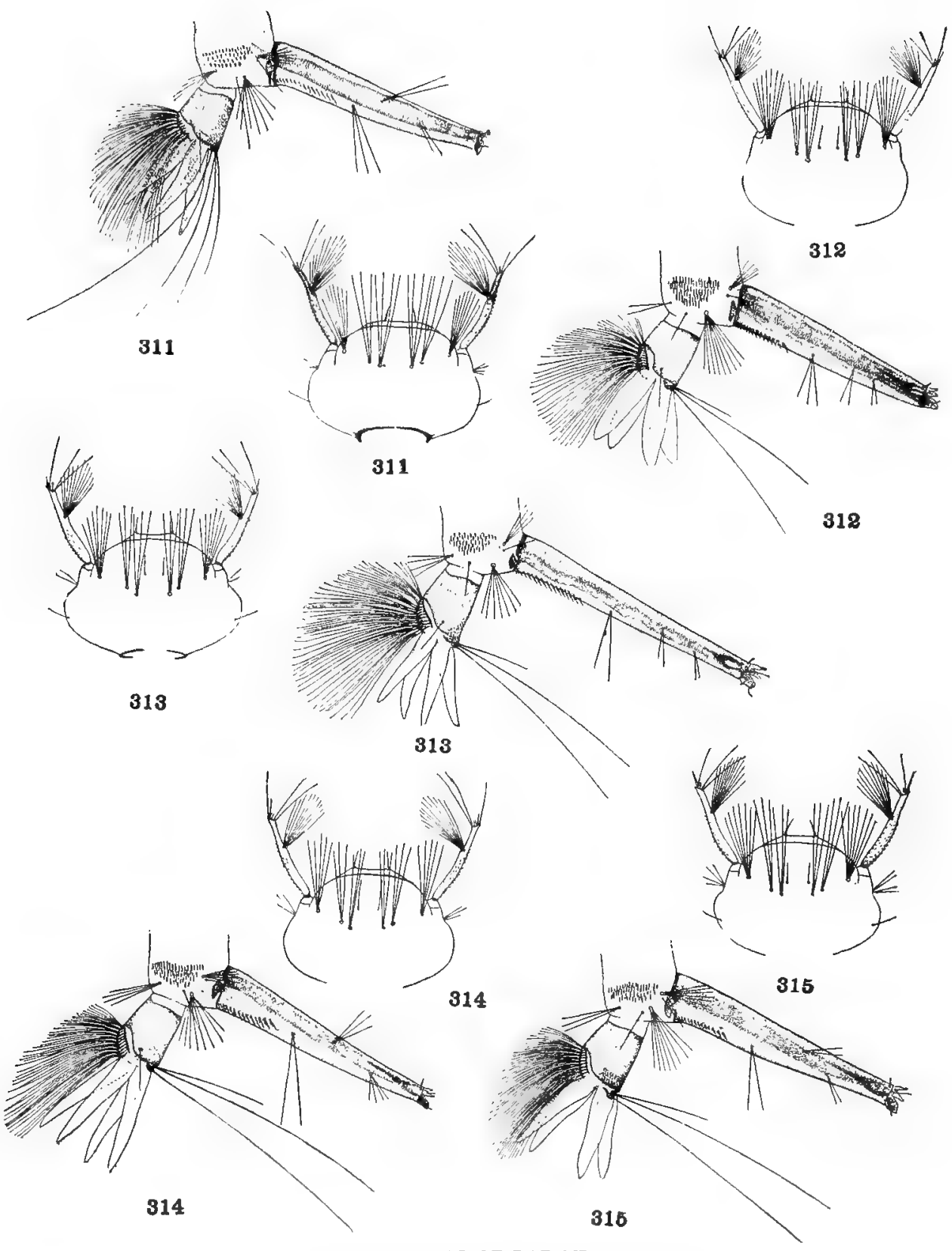

DETAILS OF LARVE.

3II. Culex equivocator $D$ yar \& Knab.

312. Culex extricator Dyar \& Knab.

313. Culex carmodyæ Dyar \& Knab.

314. Culex jubilator Dyar \& Knab.

315. Culex mollis Dyar \& Knab. 


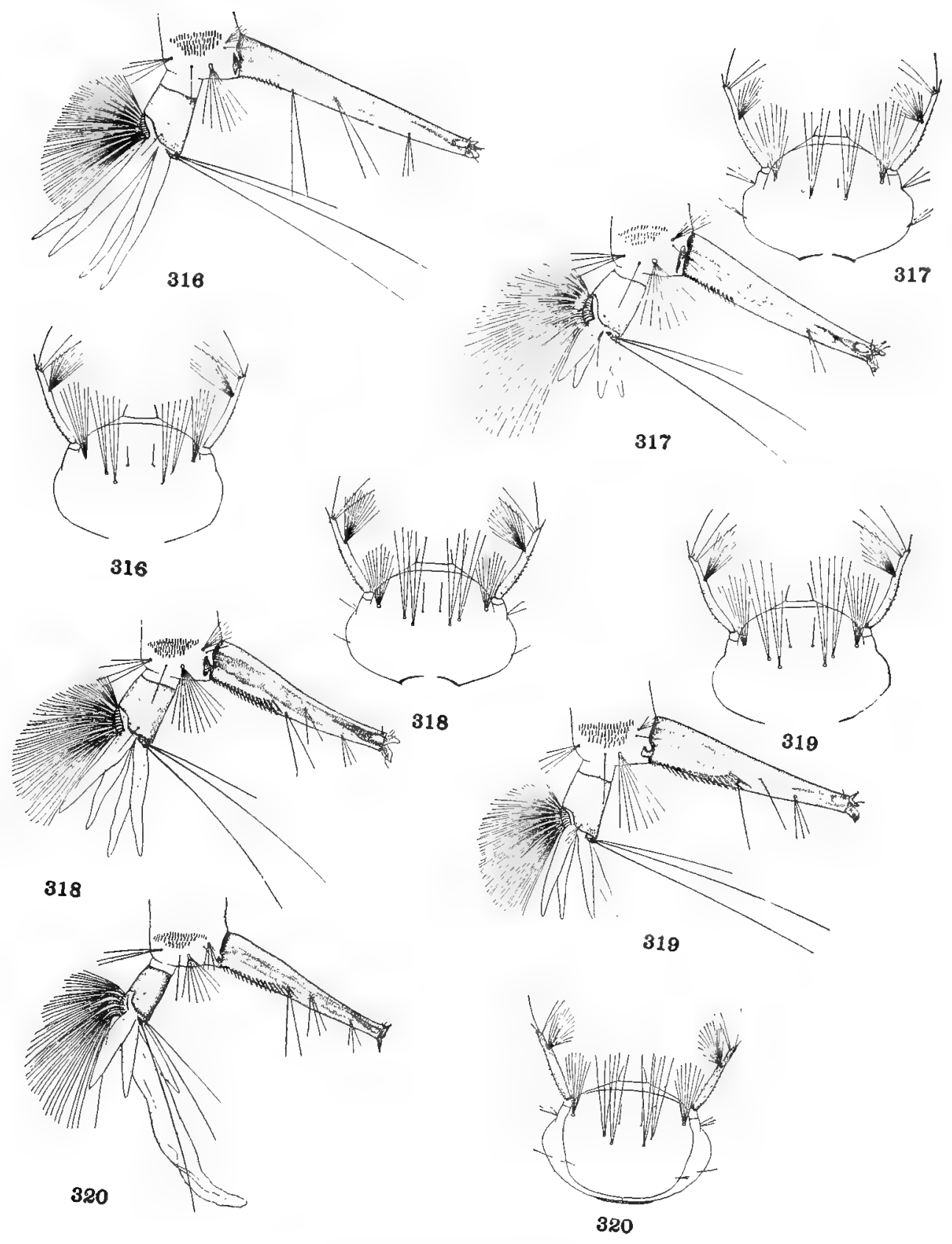

DETAILS OF LARVÆ.

316. Culex mortificator Dyar \& Knab.

317. Culex declarator Dyar \& Knab.

318. Culex inquisitor Dyar \& $K n a b$.

319. Culex proclamator Dyar \& Knab.

320. Culex revelator Dyar \& Knab. 



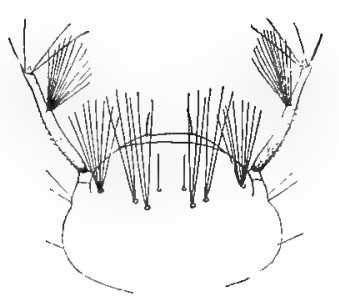

321

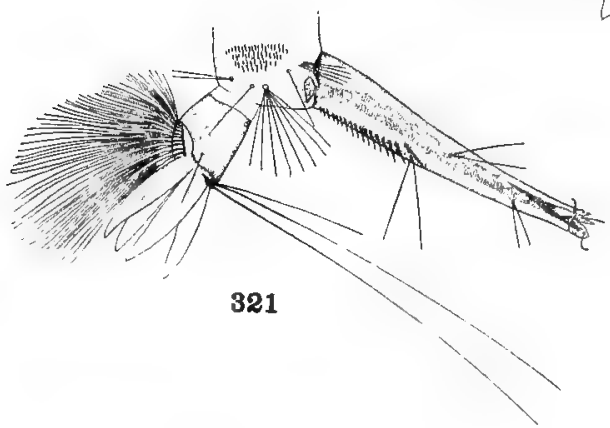

322
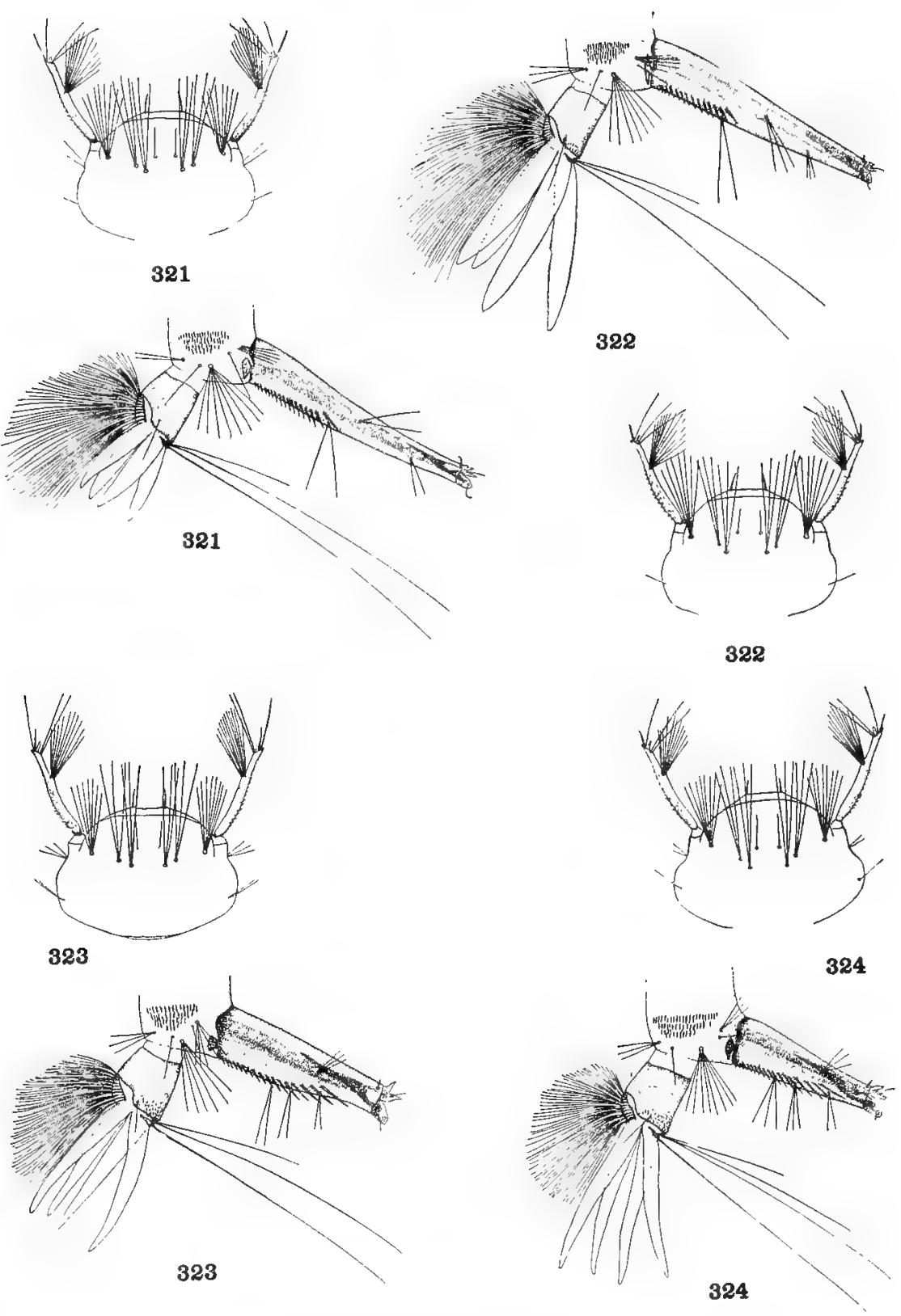

DETAILS OF LARV

32I. Culex dictator Dyar \& Knab.

322. Culex vindicator Dyar \& $K n a b$.

323. Culex reflector Dyar \& $K n a b$.

324. Culex interrogator Dyar \& Knab. 


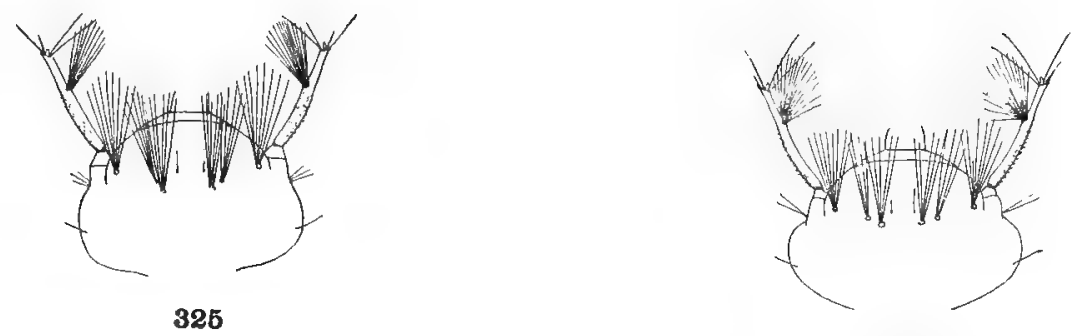

326
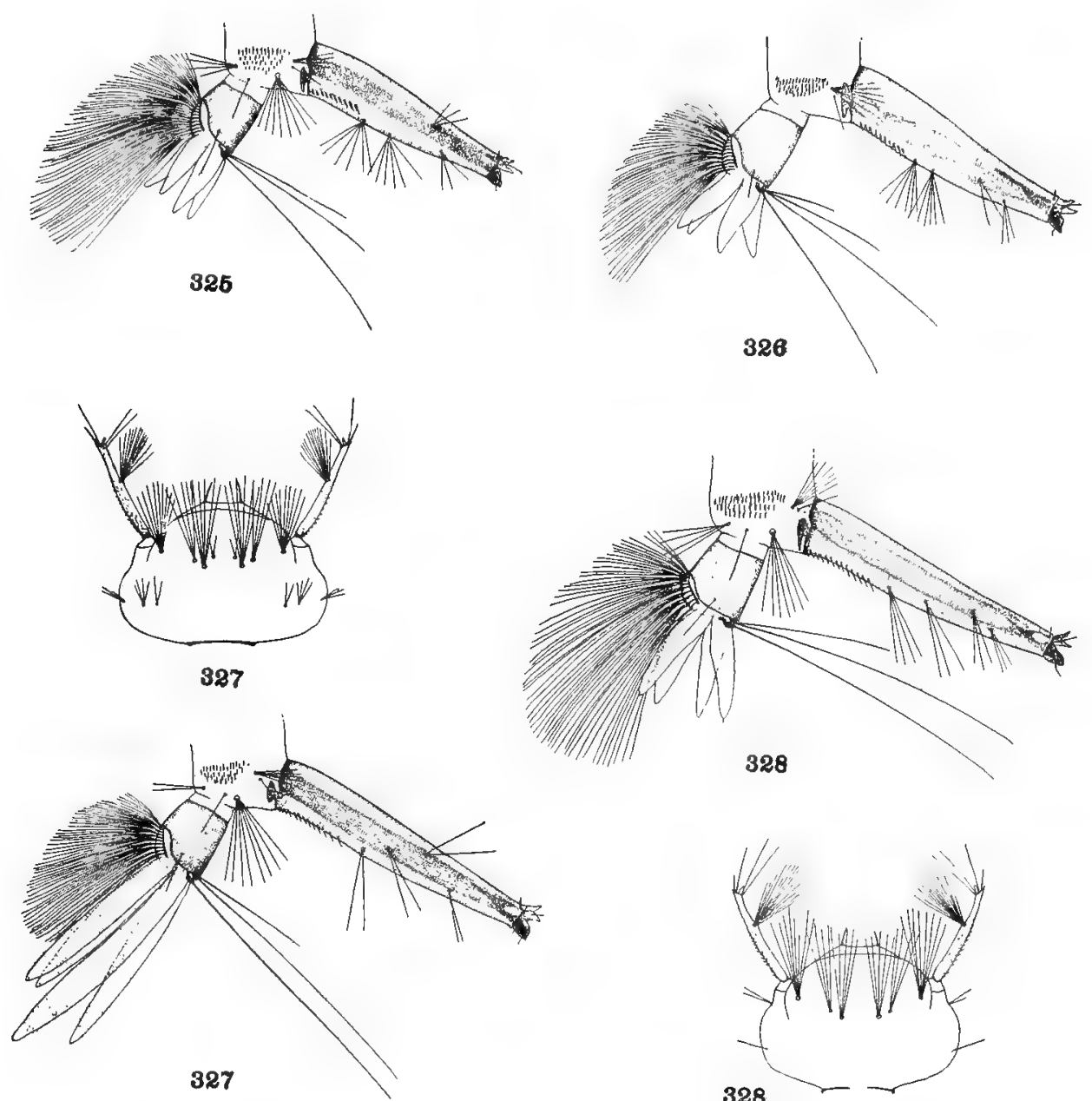

DETAILS OF LARVA.

325. Culex revocator Dyar \& Knab.

326. Culex comitatus Dyar \& Knab.

327. Culex pinarocampa Dyar \& Knab.

328. Culex pipiens Linnaus. 


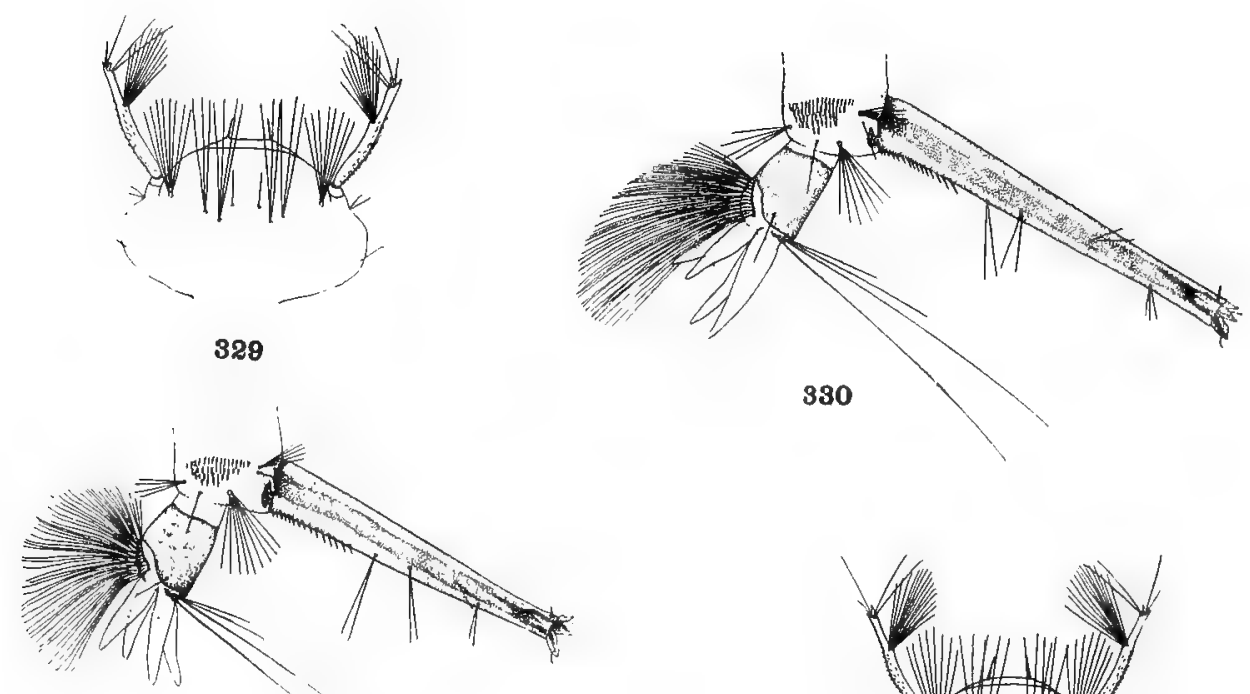

329

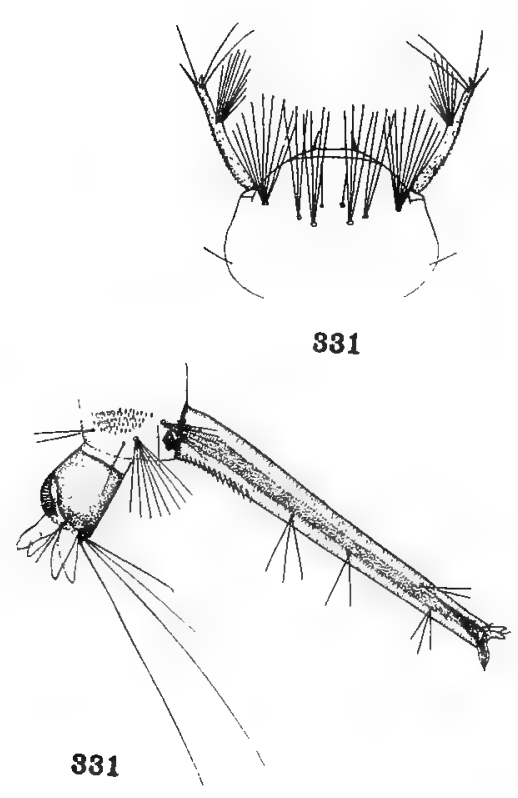

331
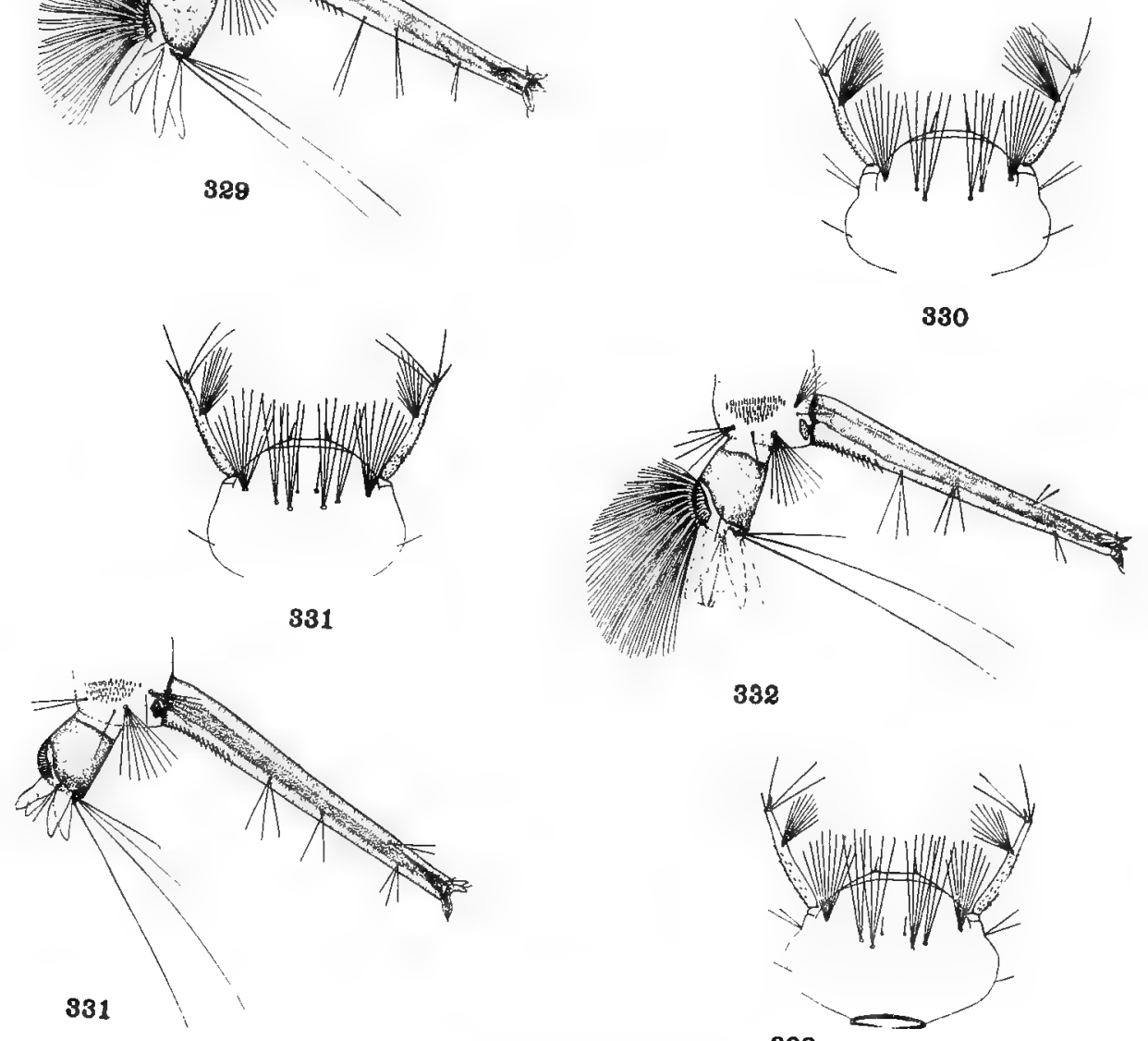

DETAILS OF LARVAE.

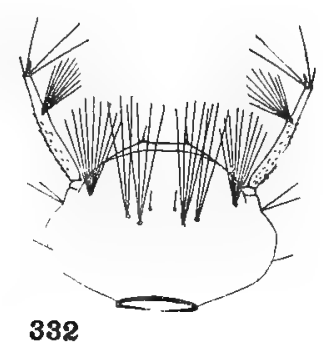

329. Culex factor $D y a r$ \& $K n a b$.

330. Culex microsquamosus Grabham.

33I. Culex eremita Howard, Dyar \& Knab.

332. Culex habilitator Dyar \& Knab. 


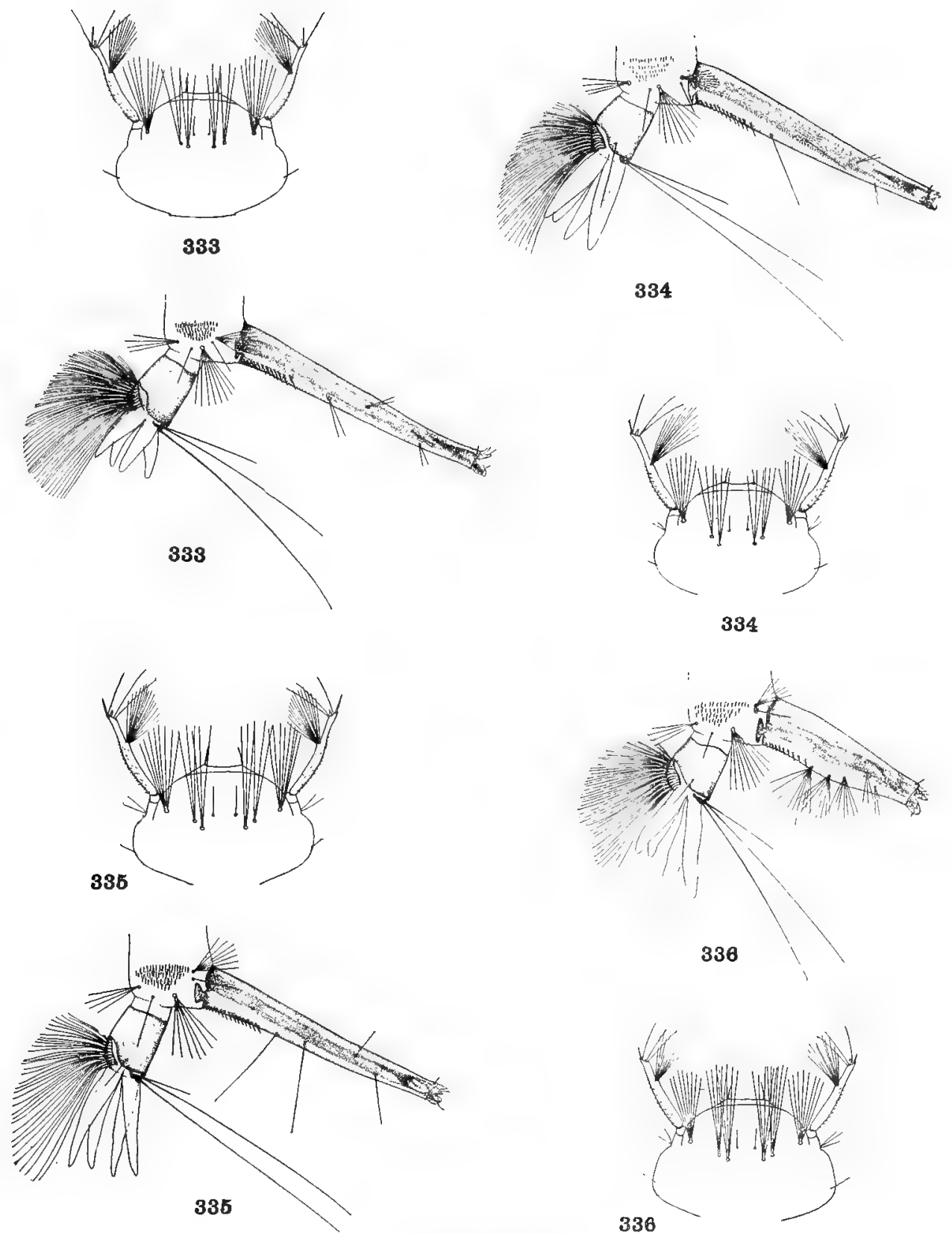

334

DETAILS OF LARVÆ.

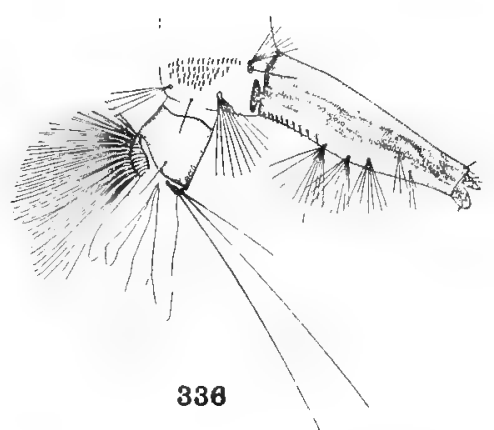

333. Culex caraibeus Howard, Dyar \& Knab.

334. Culex proximus Dyar \& $K n a b$.

335. Culex similis Theobald.

336. Culex barbarus Dyar \& Knab. 


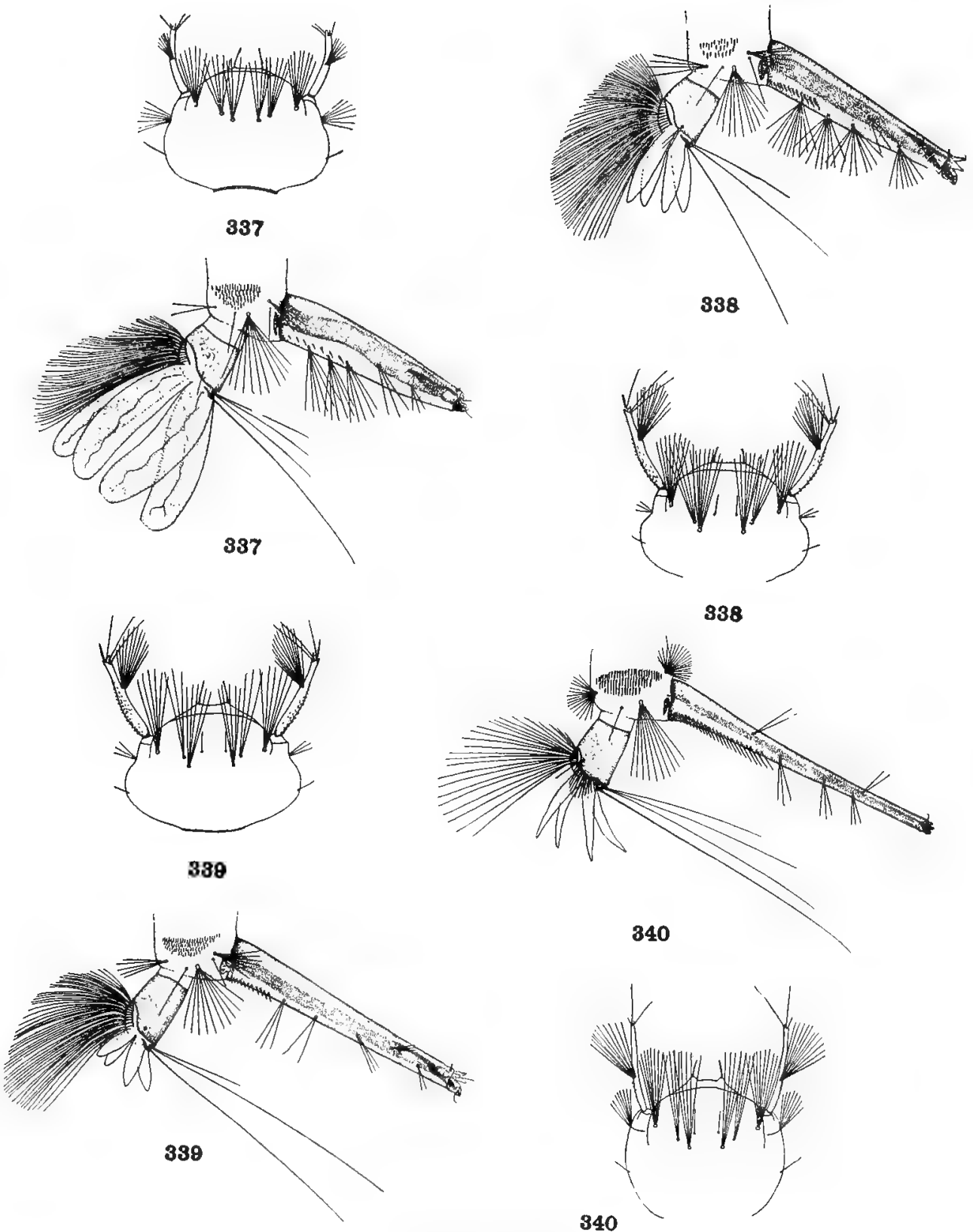

338

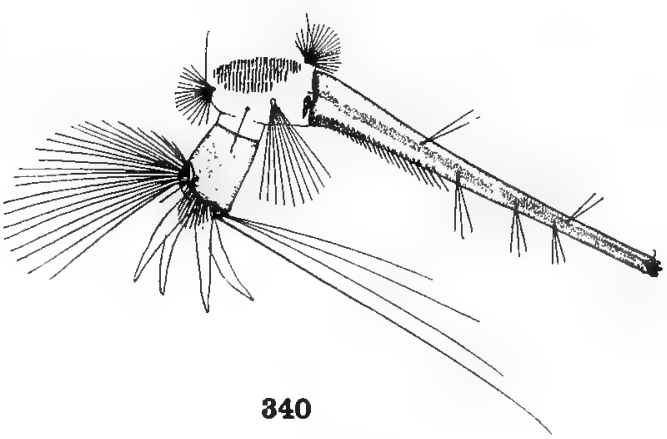

DETAILS OF LARV

337. Culex janitor Theobald.

338. Culex stigmatosoma Dyar.

339. Culex erythrothorax Dyar.

340. Culex bisulcatus Coquillett. 


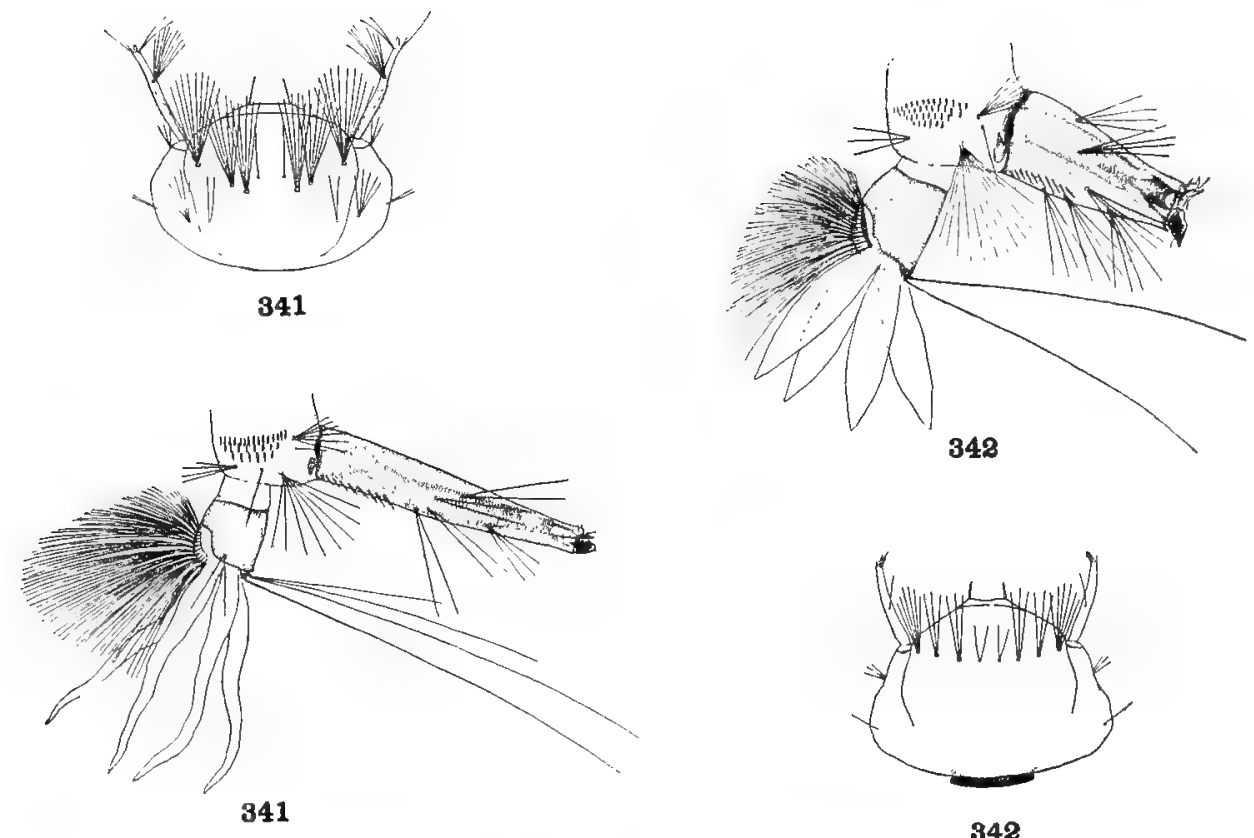

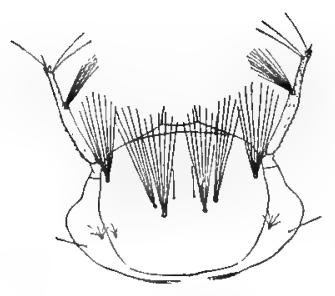

343

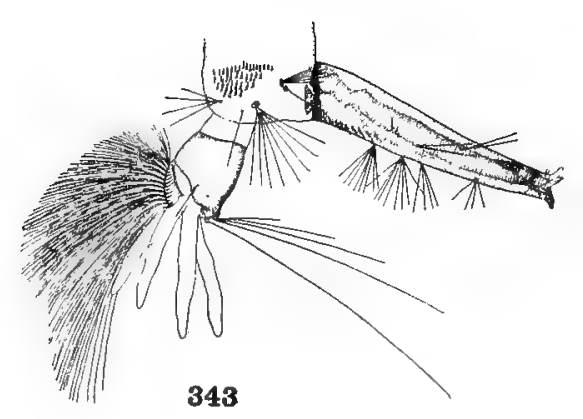

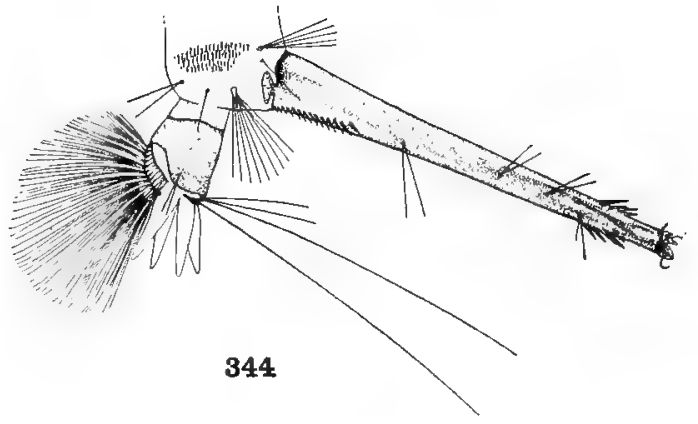

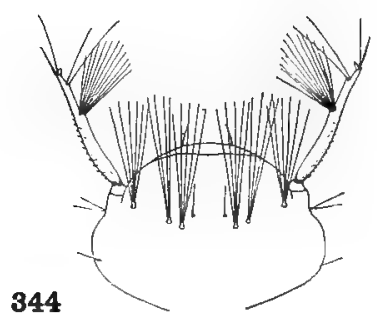

DETAILS OF LARV

34I. Culex stenolepis Dyar \& Knab.

342. Culex corniger Theobald.

343. Culex eumimetes Dyar \& $K n a b$.

344. Culex coronator Dyar \& Knab.

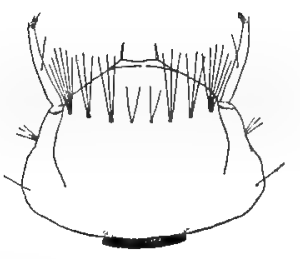

342 



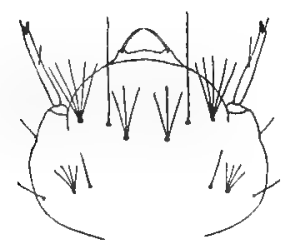

345
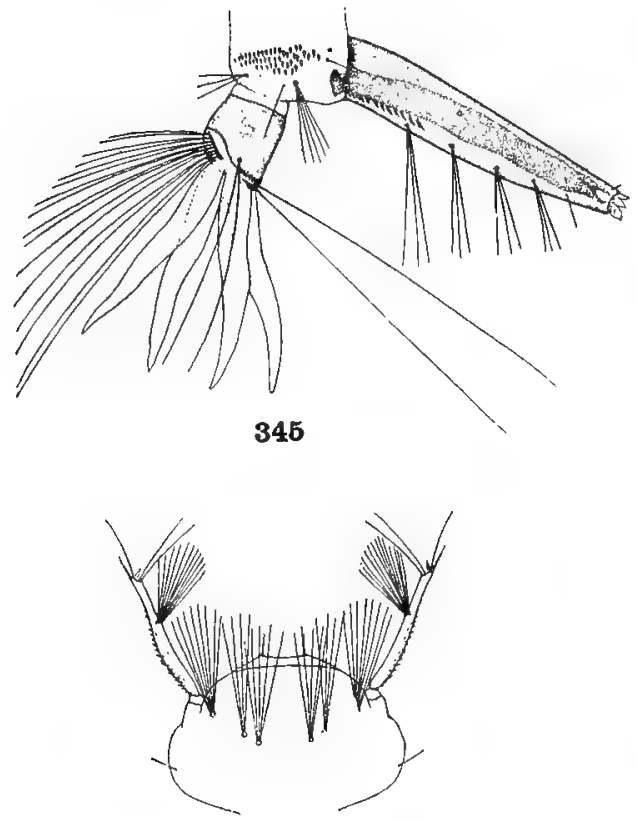

347

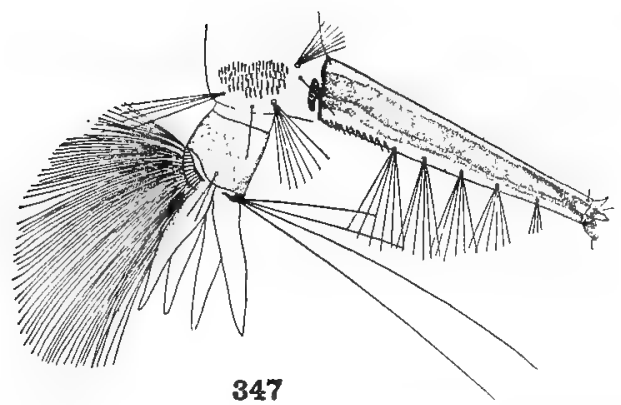

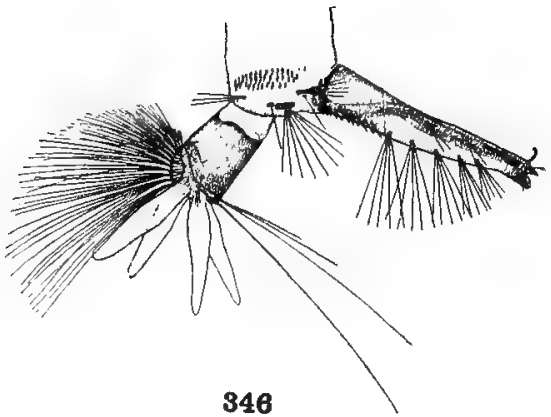

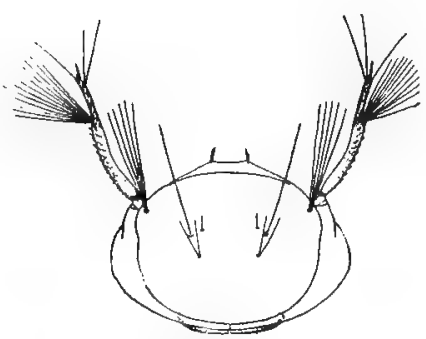

346

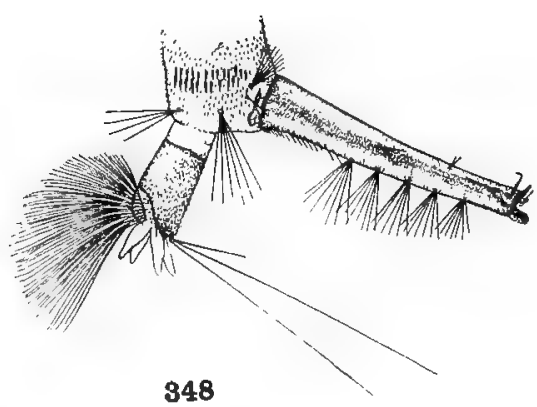

348

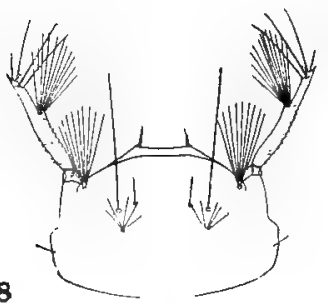

DETAILS OF LARVA.

345. Culex azymus Dyar \& $K n a b$.

346. Culex conspirator Dyar \& Knab.

347. Culex tarsalis Coquillett.

348. Culex abominator Dyar \& Knab. 


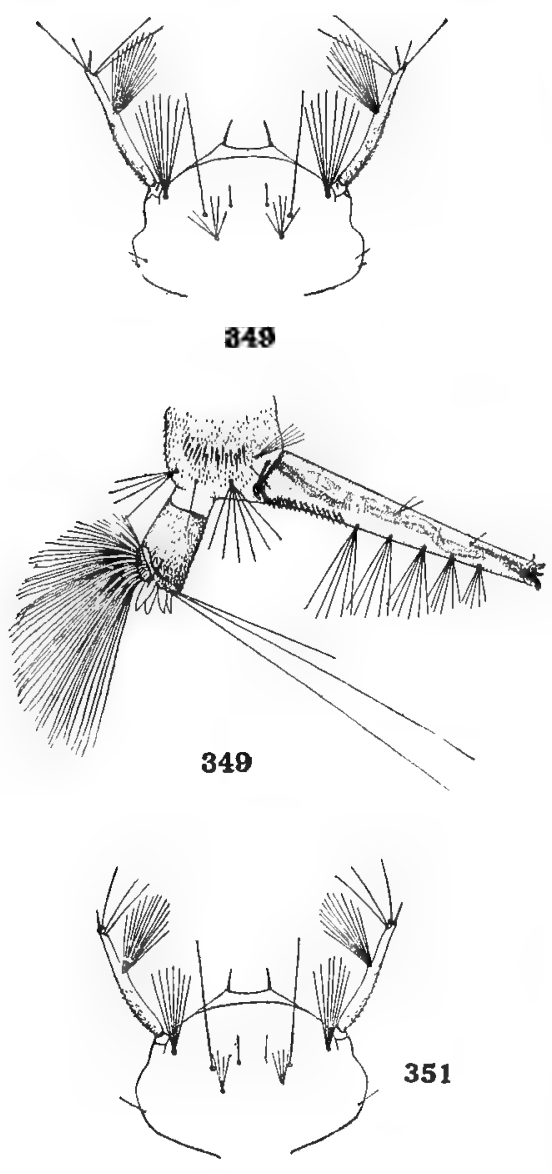

351

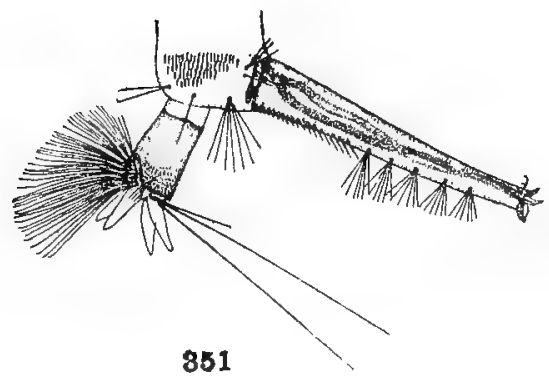

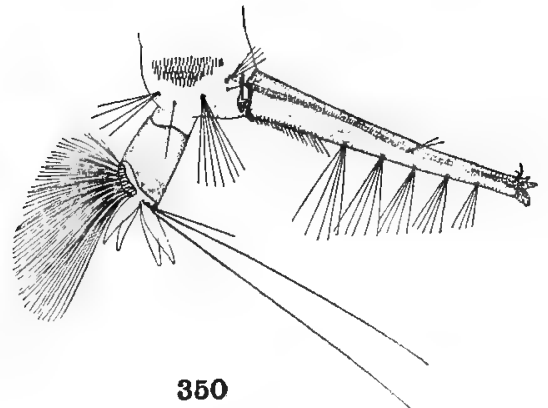
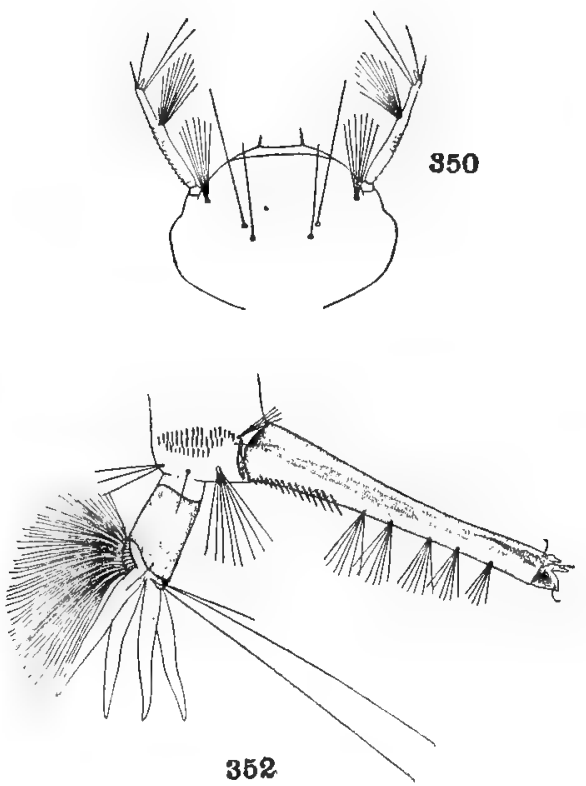

352

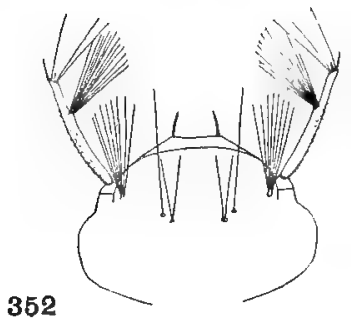

DETAILS OF LARVIE.

349. Culex erraticus Dyar \& Knab.

350. Culex gravitator Dyar \& Knab.

351. Culex investigator Dyar \& Knab.

352. Culex simulator Dyar \& Knab. 



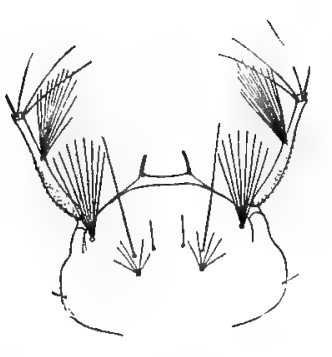

353
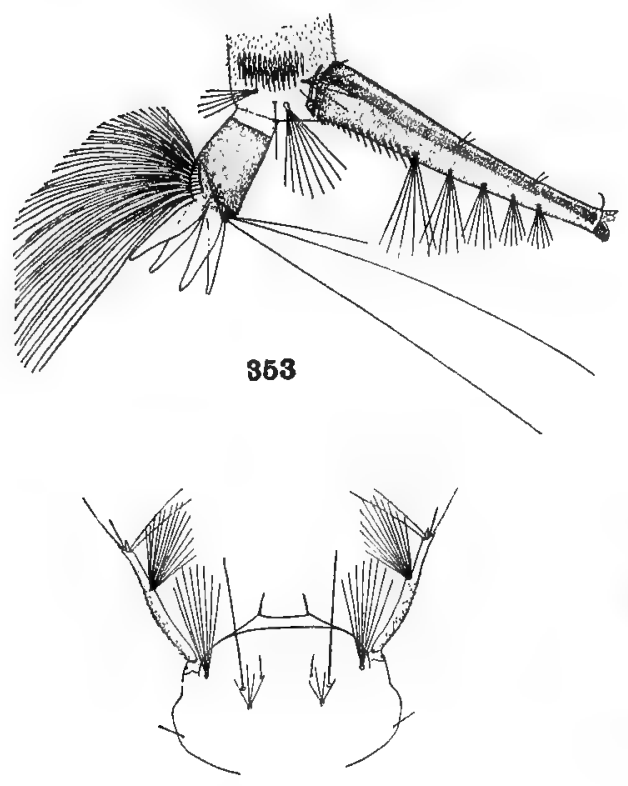

355

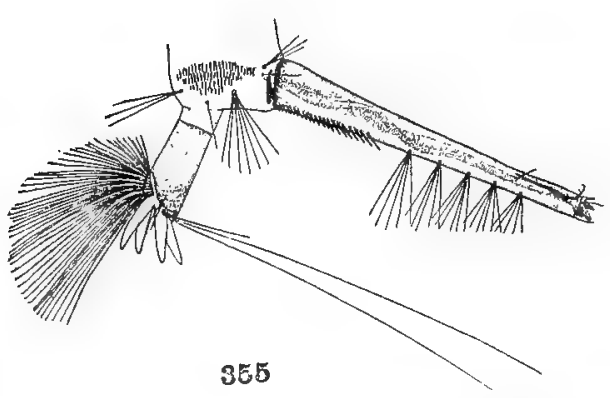

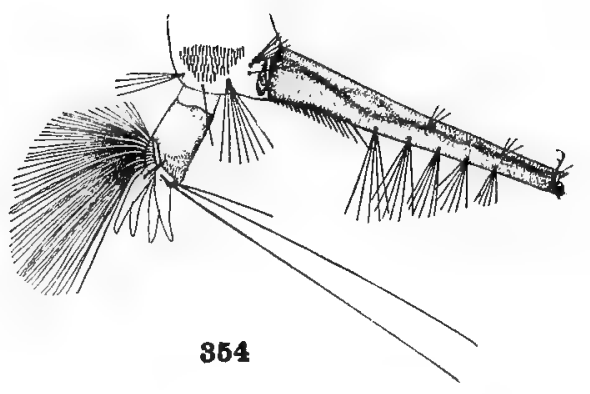

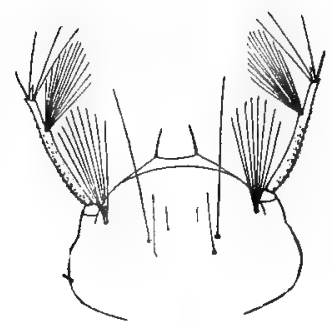

$\mathbf{3 5 4}$
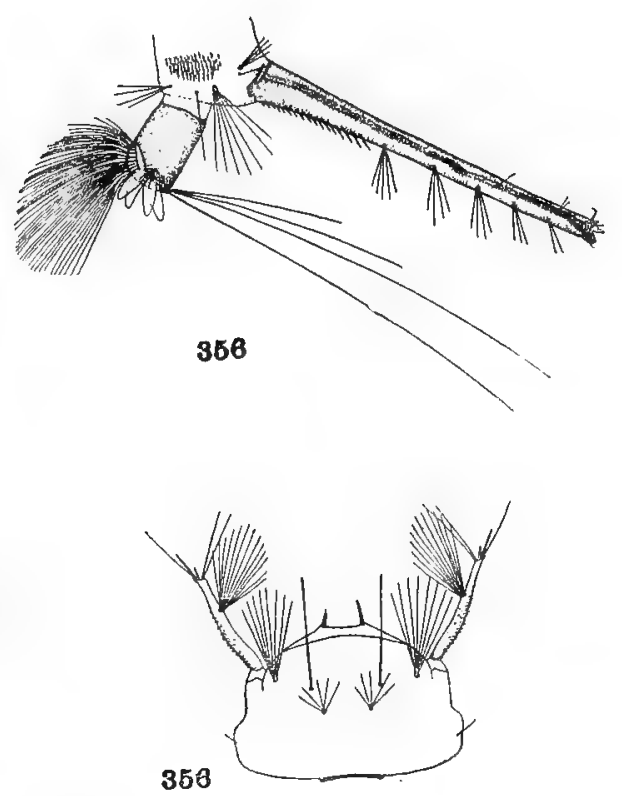

\section{DETAILS OF LARVIE.}

353. Culex leprincei Dyar \& $K n a b$.

354. Culex elevator Dyar \& Knab.

355. Culex inhibitator Dyar \& Knab.

356. Culex atratus Theobald. 


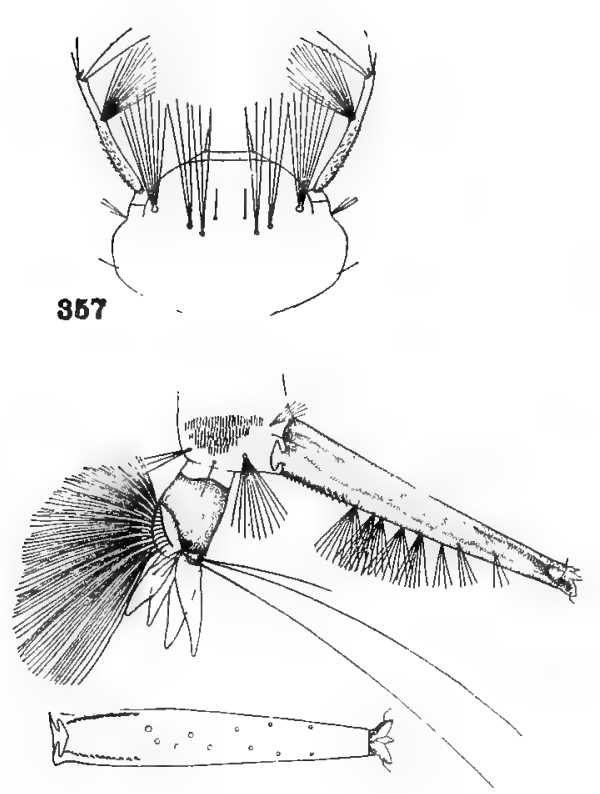

357

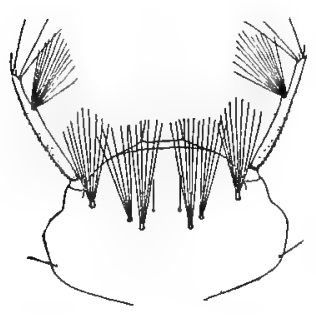

358

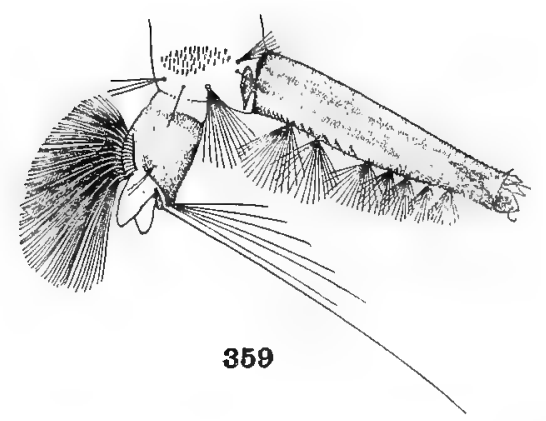

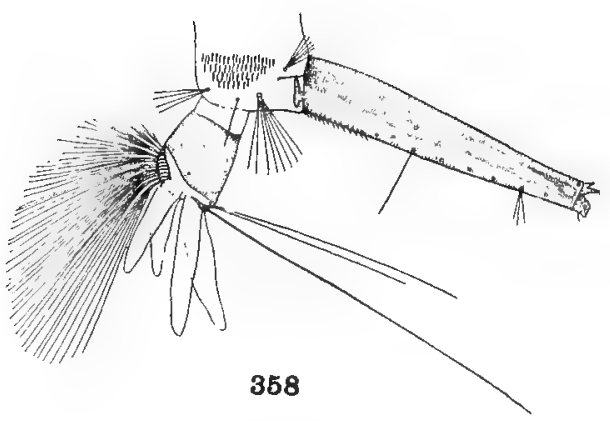

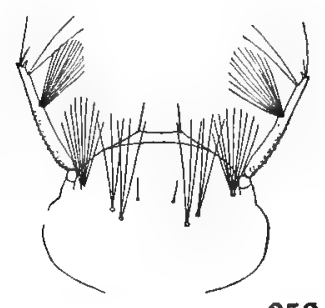

868
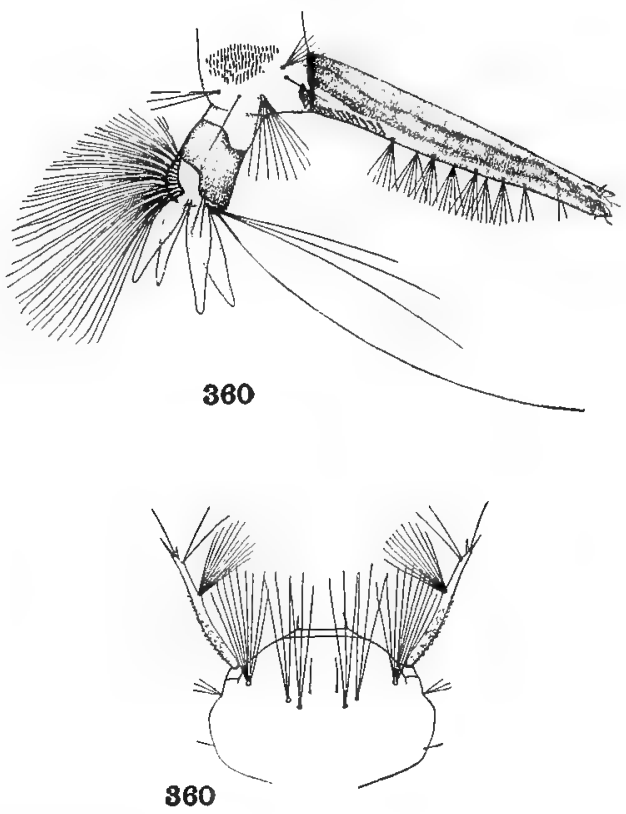

DETAILS OF LARVA.

357. Culex lamentator Dyar \& Knab.

358. Culex toweri Dyar \& $K n a b$.

359. Culex bahamensis Dyar \& Knab.

360. Culex secutor Theobald. 



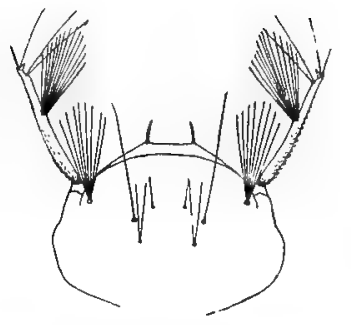

381

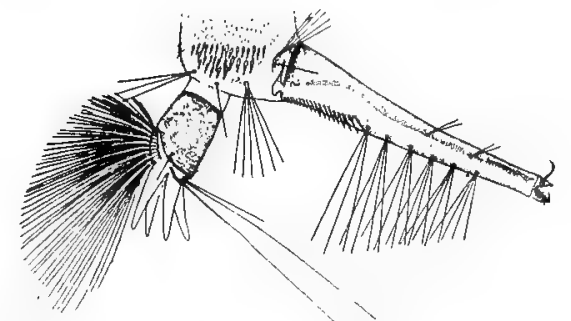

361
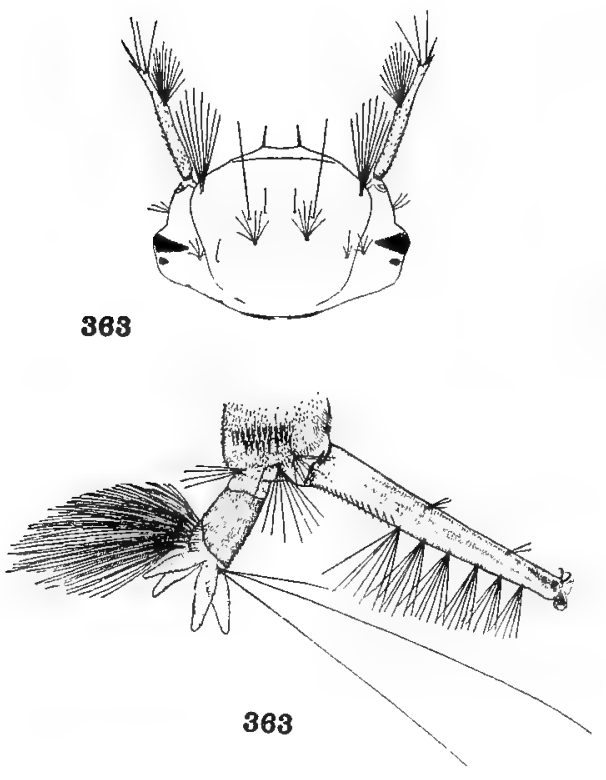

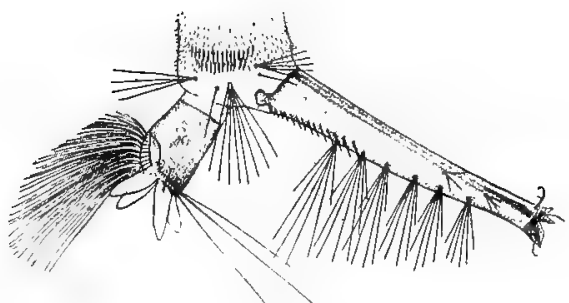

362

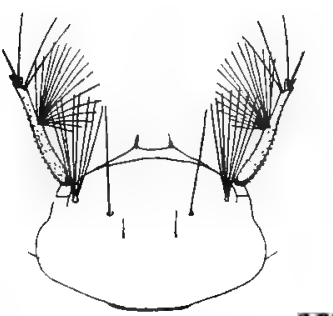

389
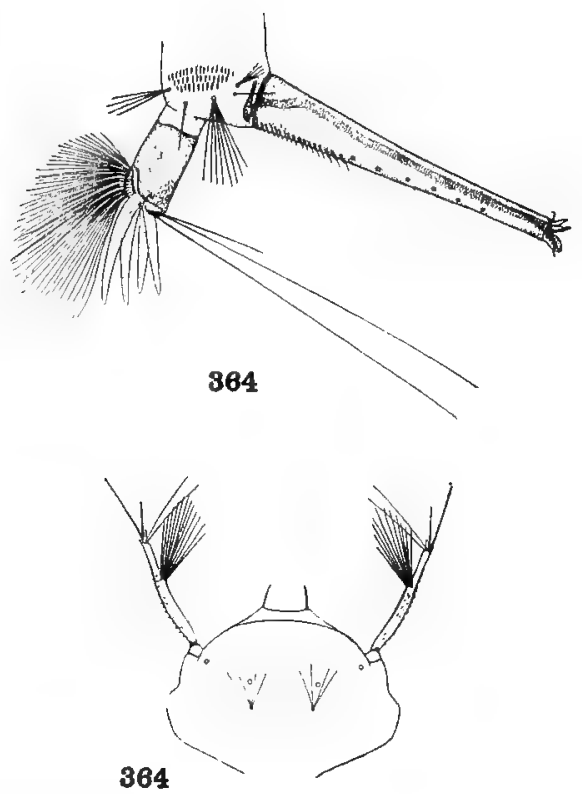

DETAILS OF LARV压.

36r. Culex mutator Dyar \& Knab.

362. Culex chrysonotum Dyar \& $K n a b$.

363. Culex trachycampa Dyar \& $K n a b$.

364. Culex bastagarius Dyar \& Knab. 



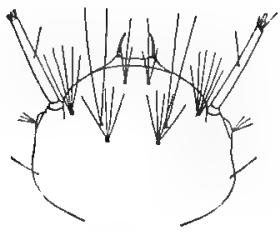

365
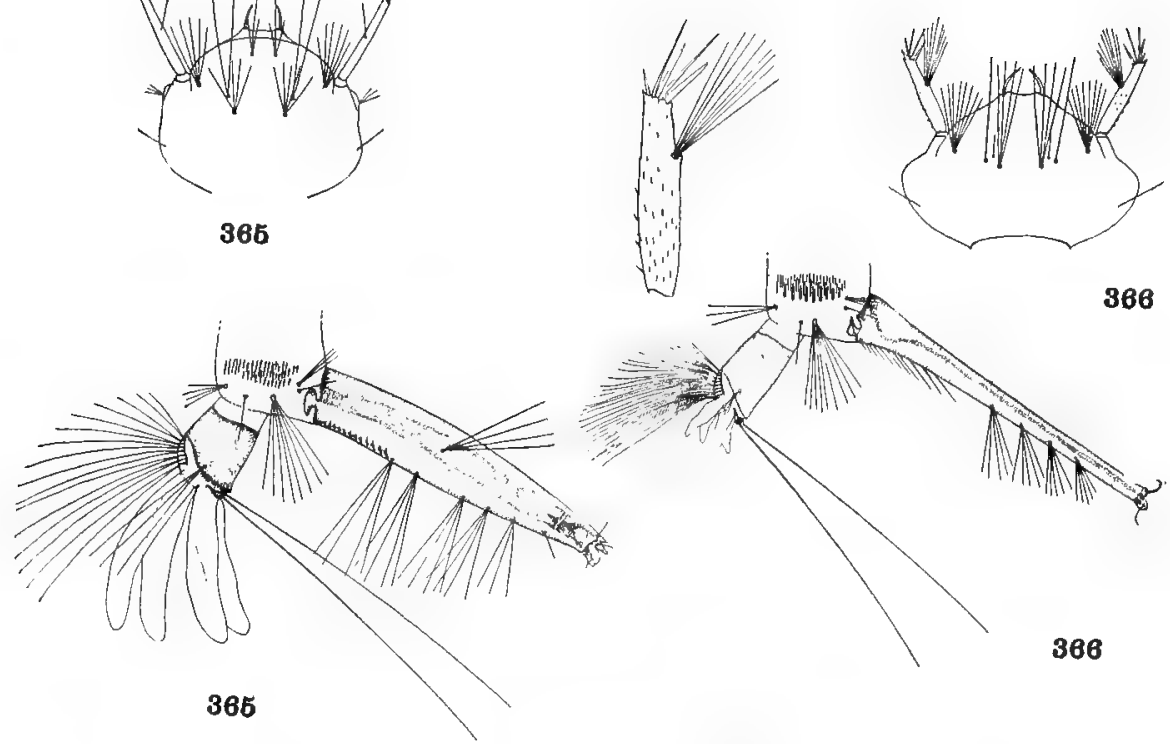

366

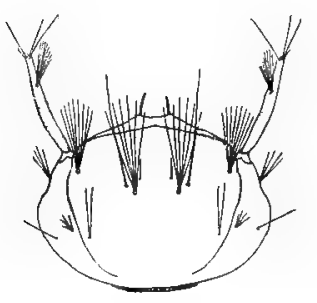

367

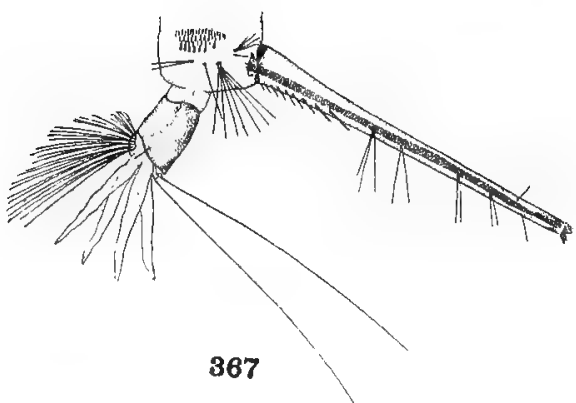

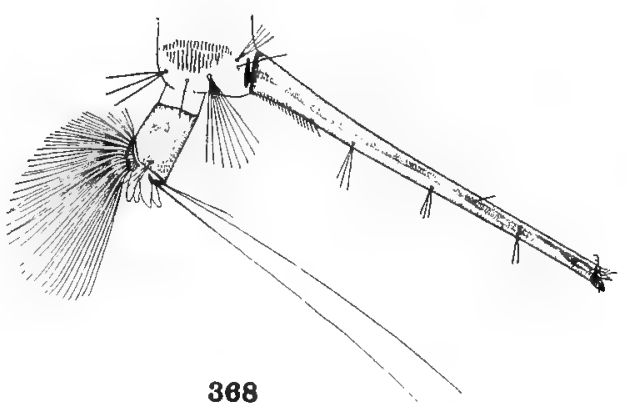

368

366

DETAILS OF LARVE.

365. Culex pleuristriatus Theobald.

366. Culex daumastocampa Dyar \& $K n a b$

367. Culex rejector Dyar \& Knab.

368. Culex carcinophilus Dyar \& Knab. 


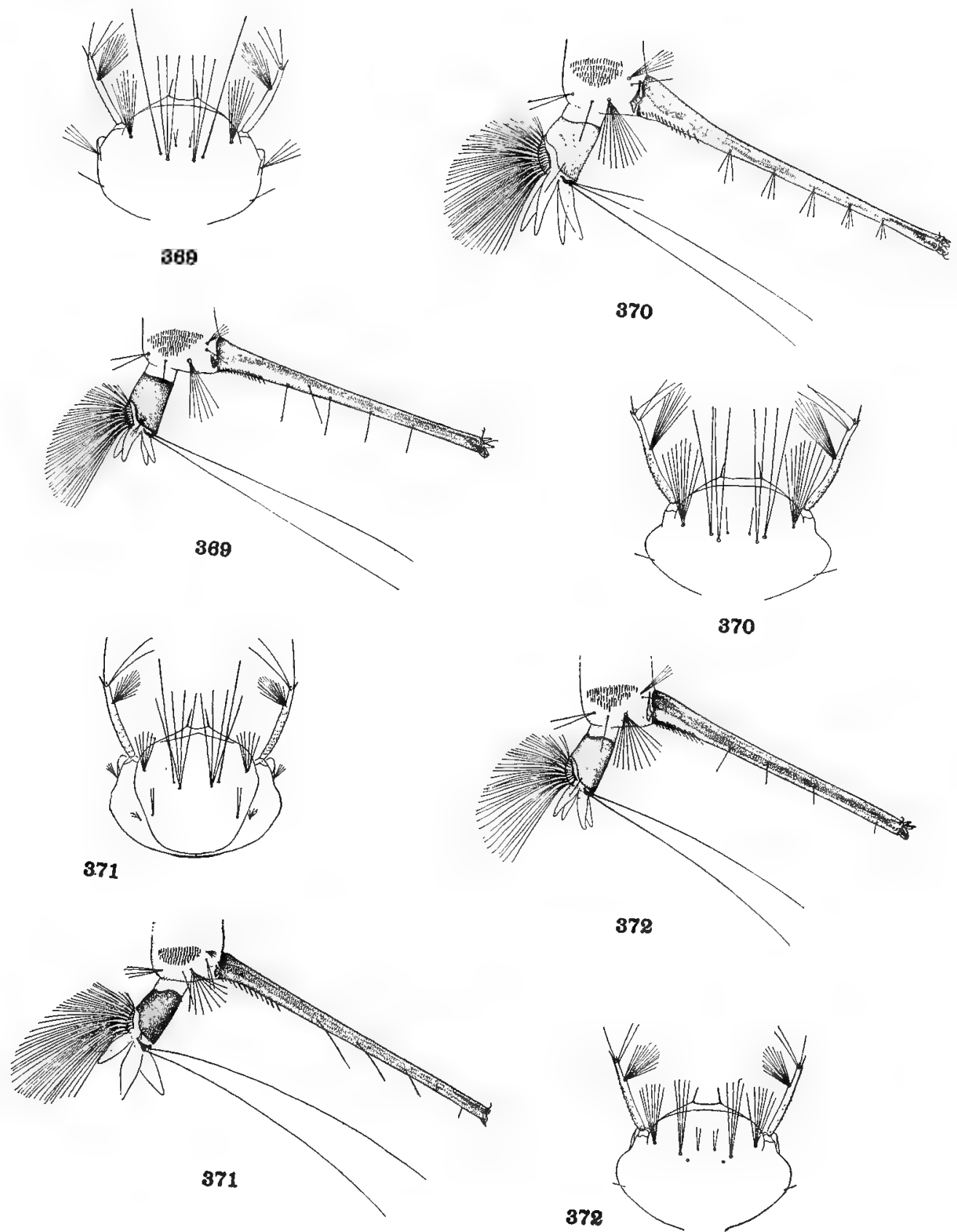

DETAILS OF LARVA.

369. Culex restrictor Dyar \& $K n a b$.

370. Culex derivator Dyar \& Knab.

371. Culex corrigani Dyar \& Knab.

372. Culex conservator Dyar \& Knab. 


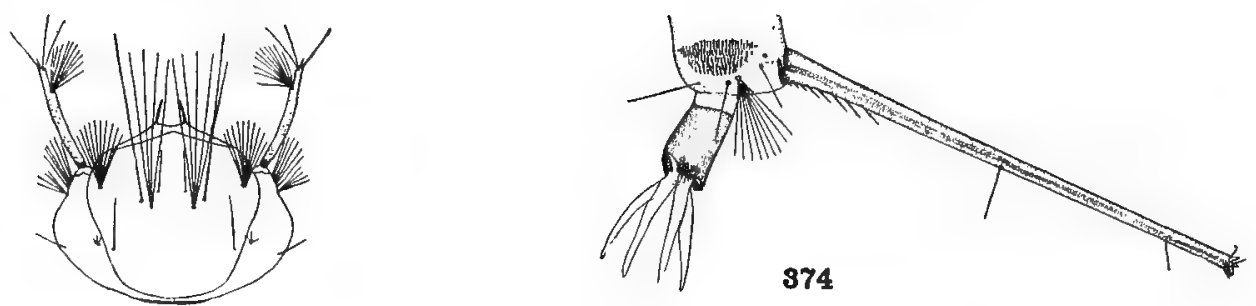

373

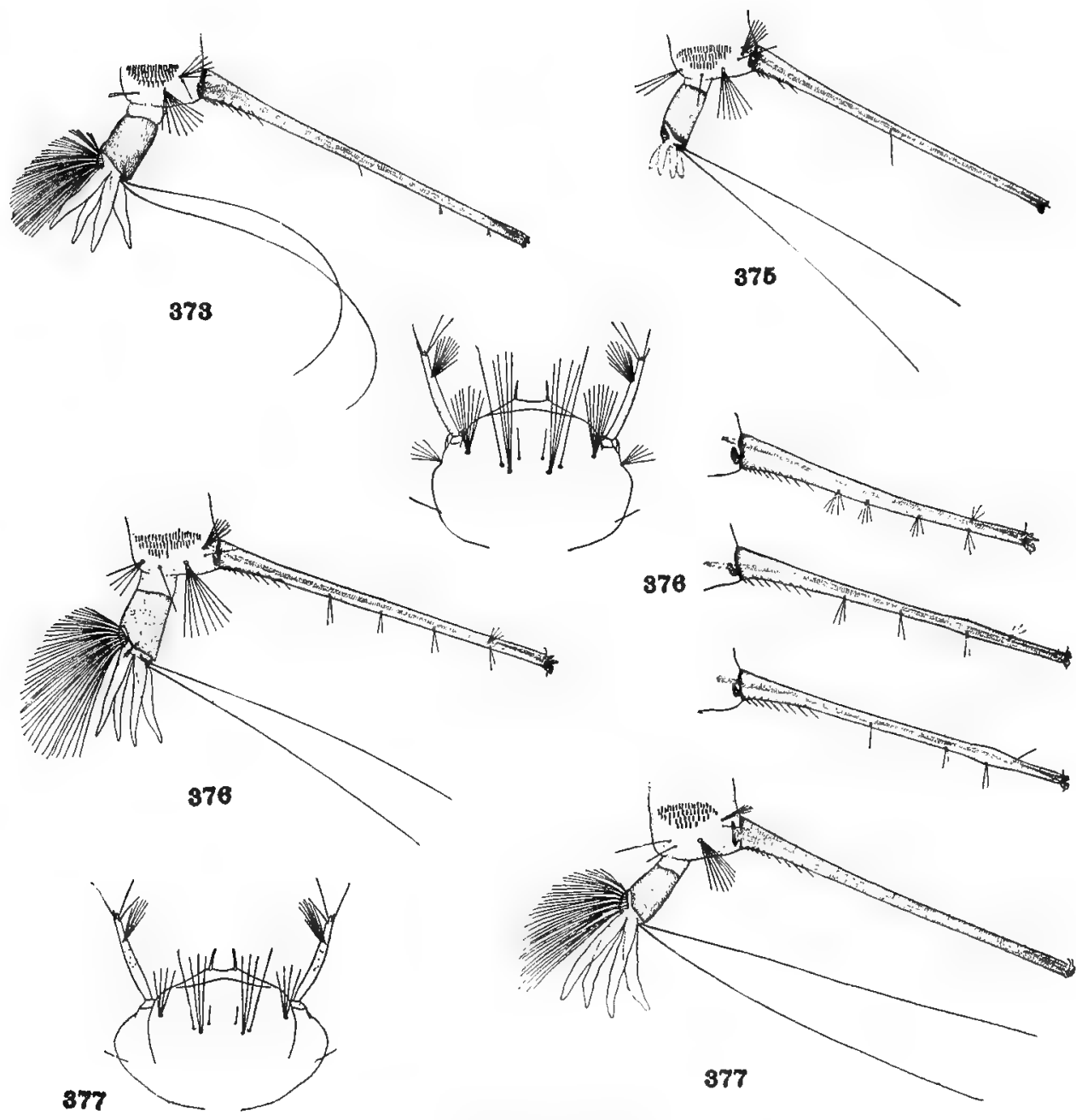

DETAILS OF LARVA.

373. Culex jenningsi Dyar \& Knab.

374. Culex consolator Dyar \& Knab.

375. Culex inimitabilis Dyar \& Knab.

376. Culex imitator Theobald.

377. Culex ocellatus Theobald. 


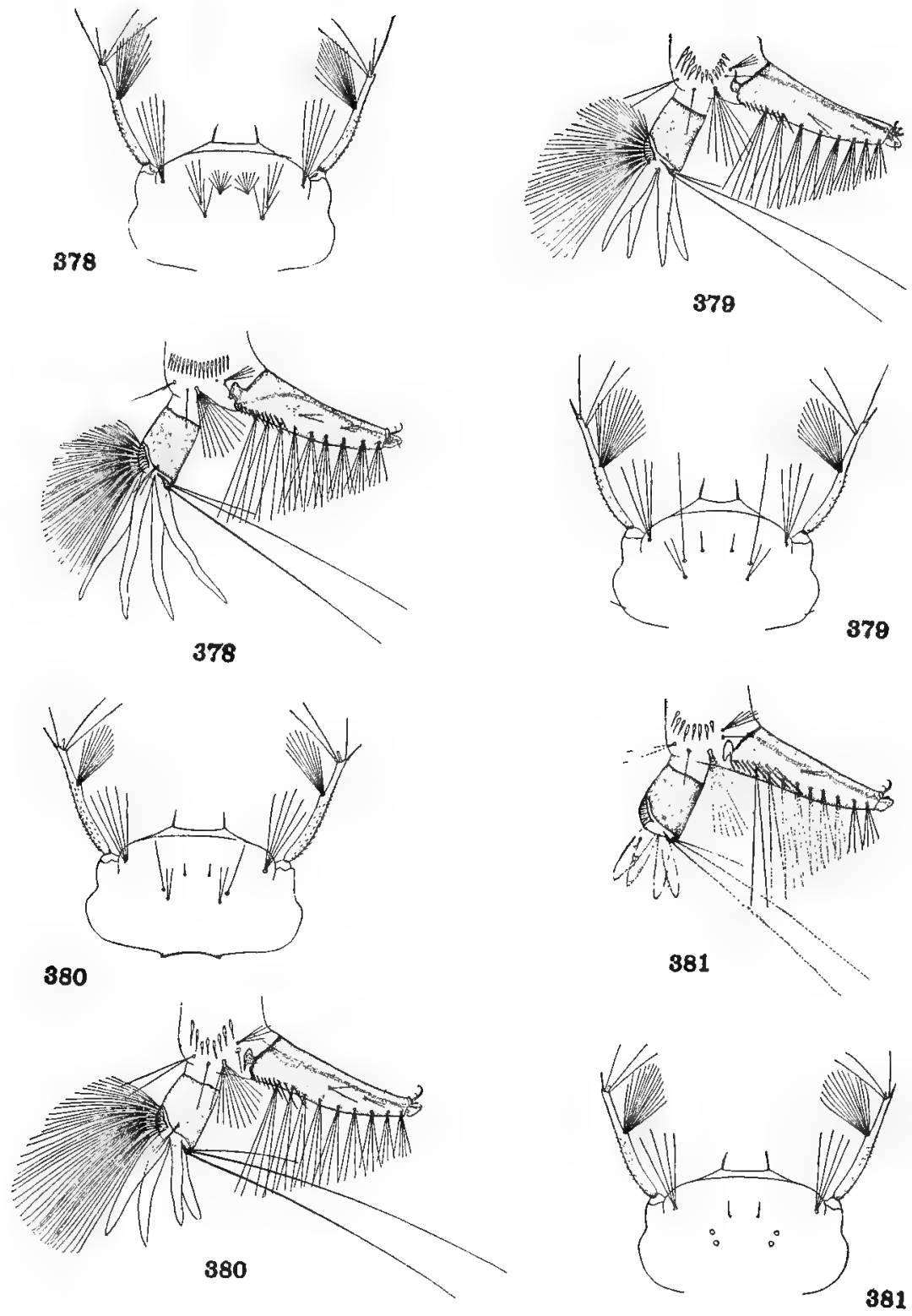

DETAILS OF LARV 现.

378. Culex caudelli Dyar \& Knab.

379. Culex pilosus Dyar \& $K n a b$.

380. Culex floridanus Dyar \& Knab.

38I. Culex agitator Dyar \& Knab. 


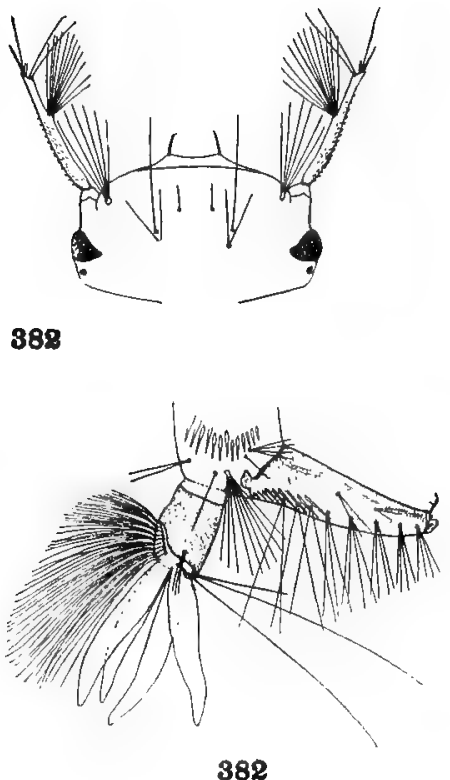

382

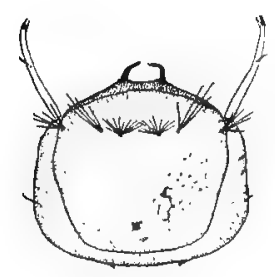

384

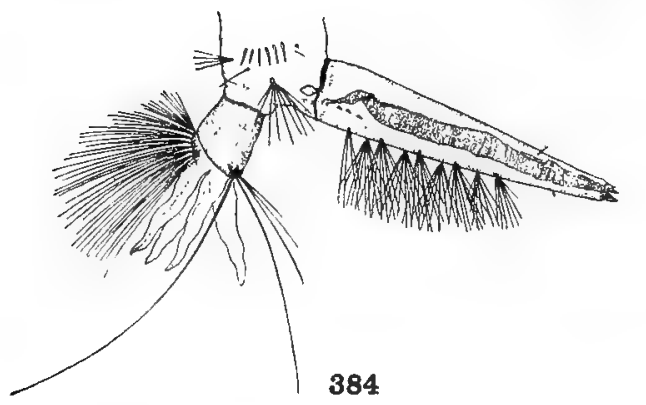

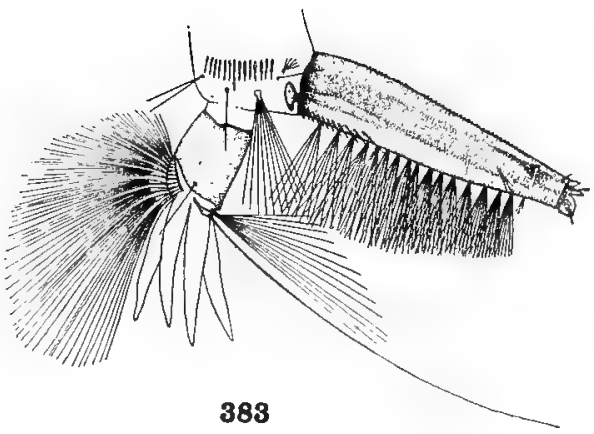

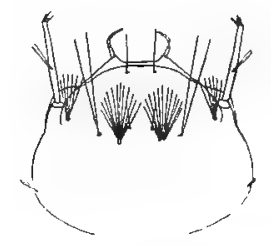

383

DETAILS OF LARVAE.

382. Culex reductor Dyar \& Knab.

383. Carrollia urichii Coquillett.

384. Carrollia iridescens Lutz.

385. Lutzia bigoti Bellardi.

385

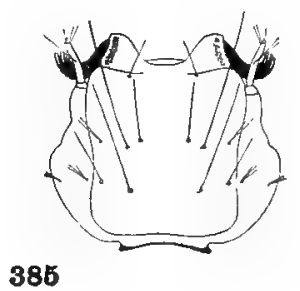




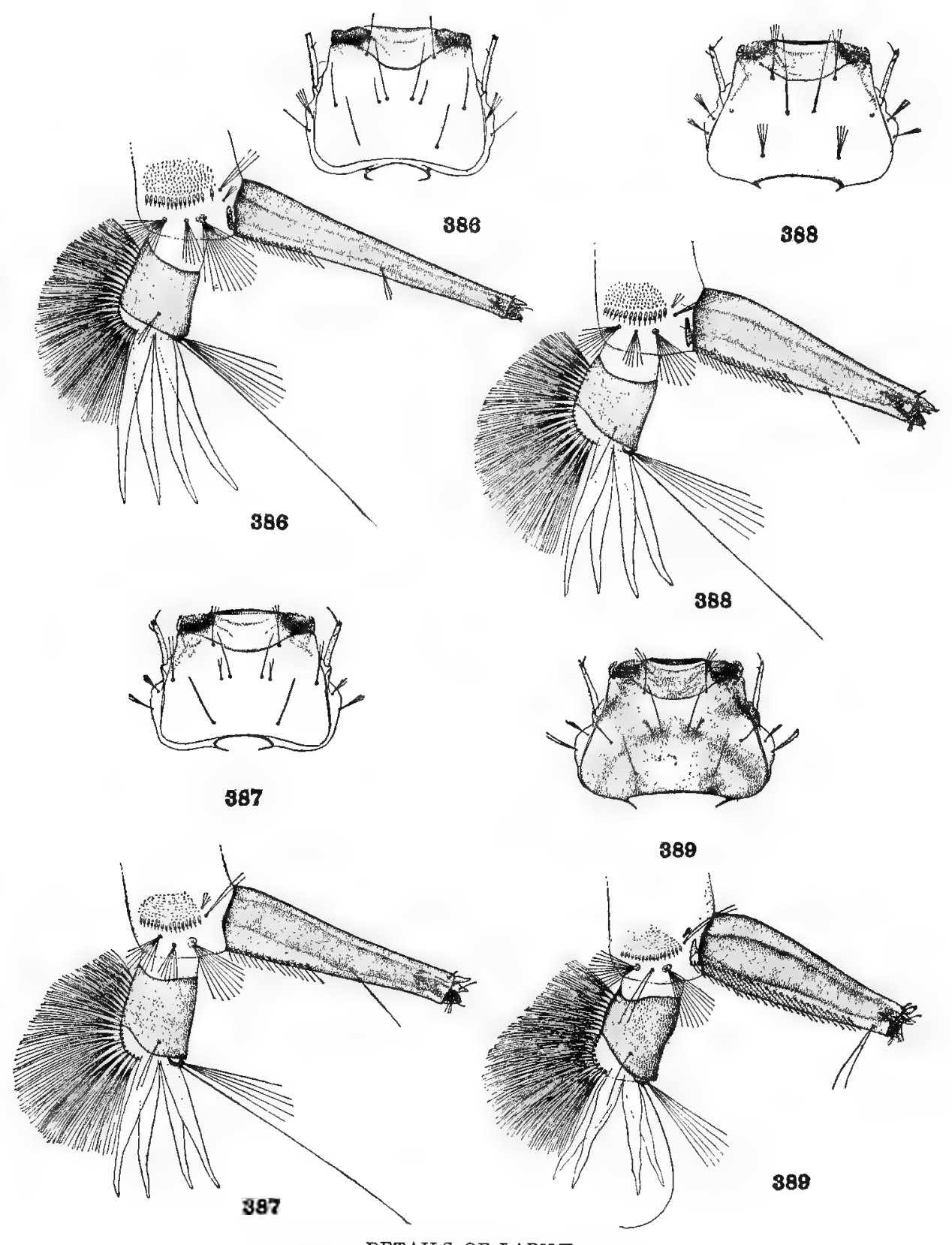

DETAILS OF LARVA.

386. Psorophora sæva Dyar \& Knab.

387. Psorophora virescens Dyar \& Knab.

388. Psorophora howardii Coquillett.

389. Psorophora cilipes Fabricius. 


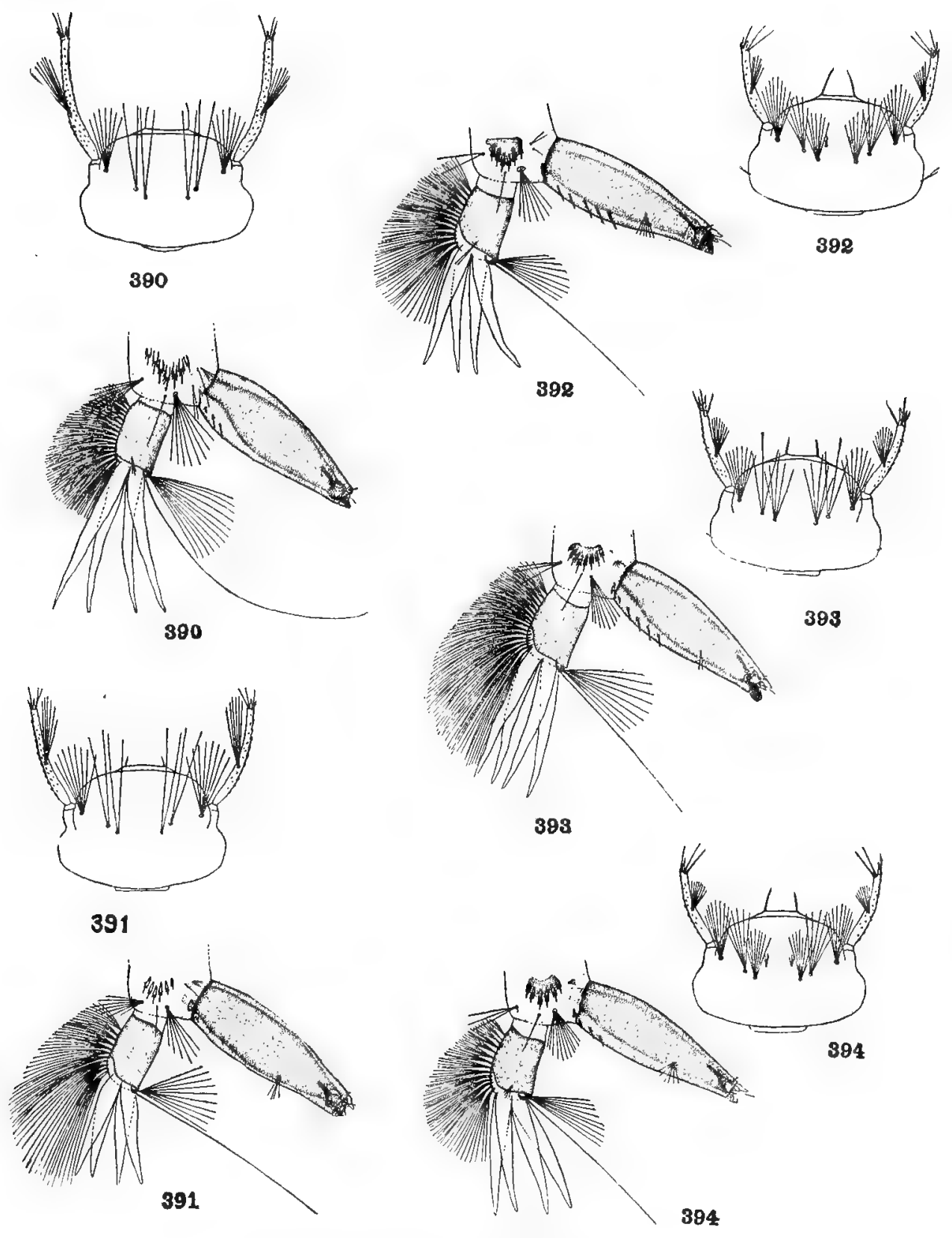

DETAILS OF LARV死.

390. Psorophora sayi Dyar \& Knab.

391. Psorophora discrucians Wiedemann.

392. Psorophora toltecum Dyar \& Knab.

393. Psorophora floridense Dyar \& $K n a b$.

394. Psorophora jamaicensis Theobald. 


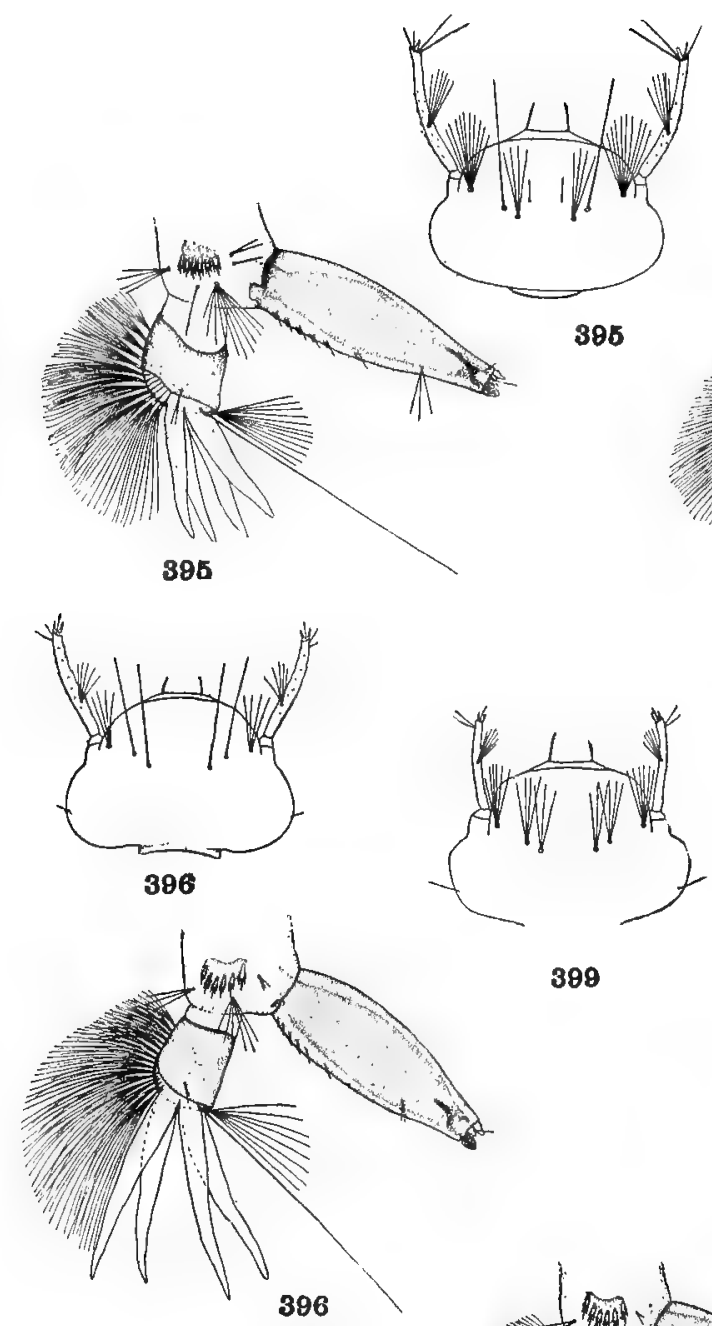

389

398
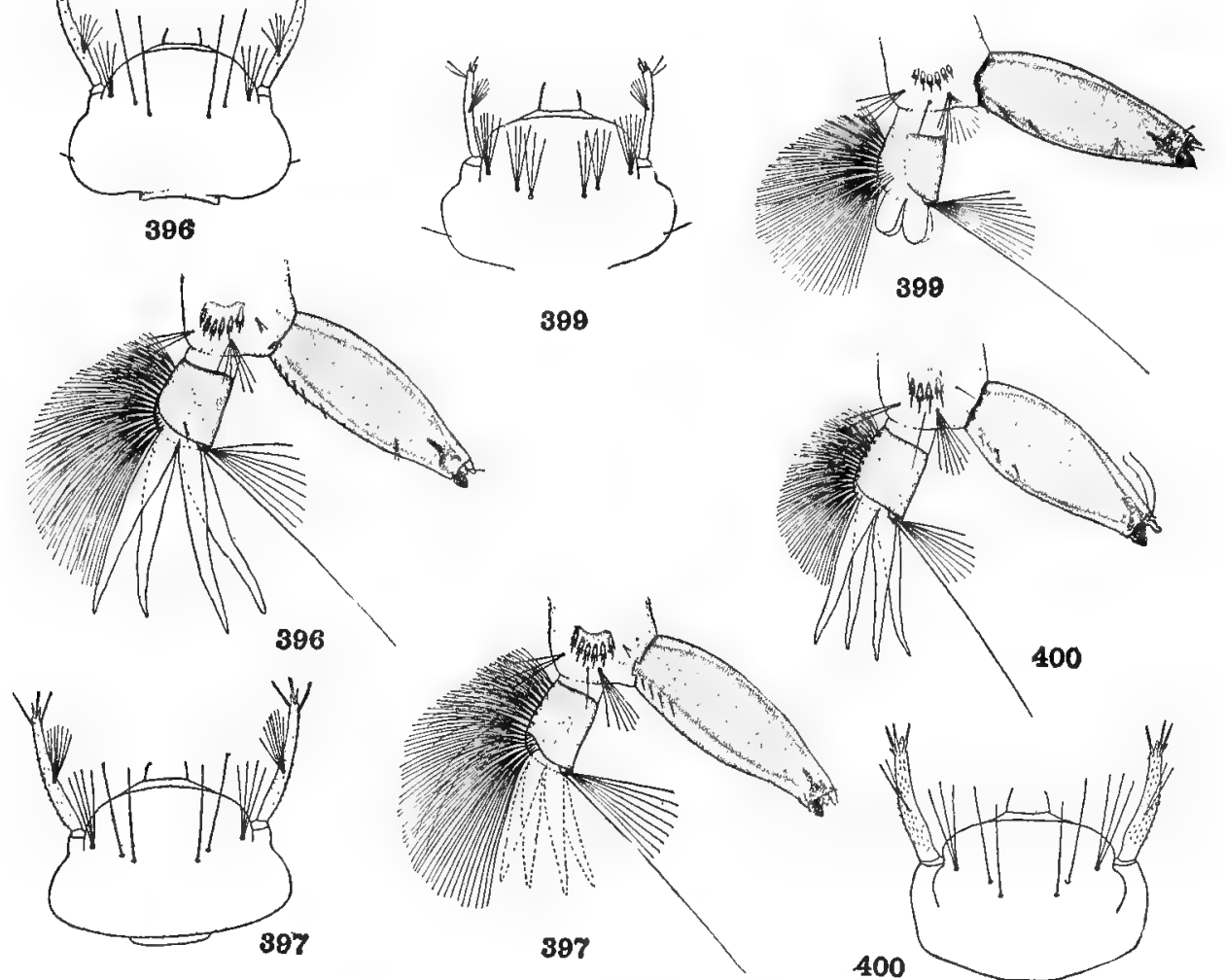

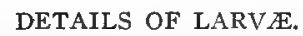

395. Psorophora infine Dyar \& Knab.

396. Psorophora pygmæa Theobald.

397. Psorophora signipennis Coquillett.

398. Psorophora haruspicus Dyar \& $K n a b$.

399. Psorophora insularius Dyar \& Knab.

400. Psorophora cyanescens Coquillett. 


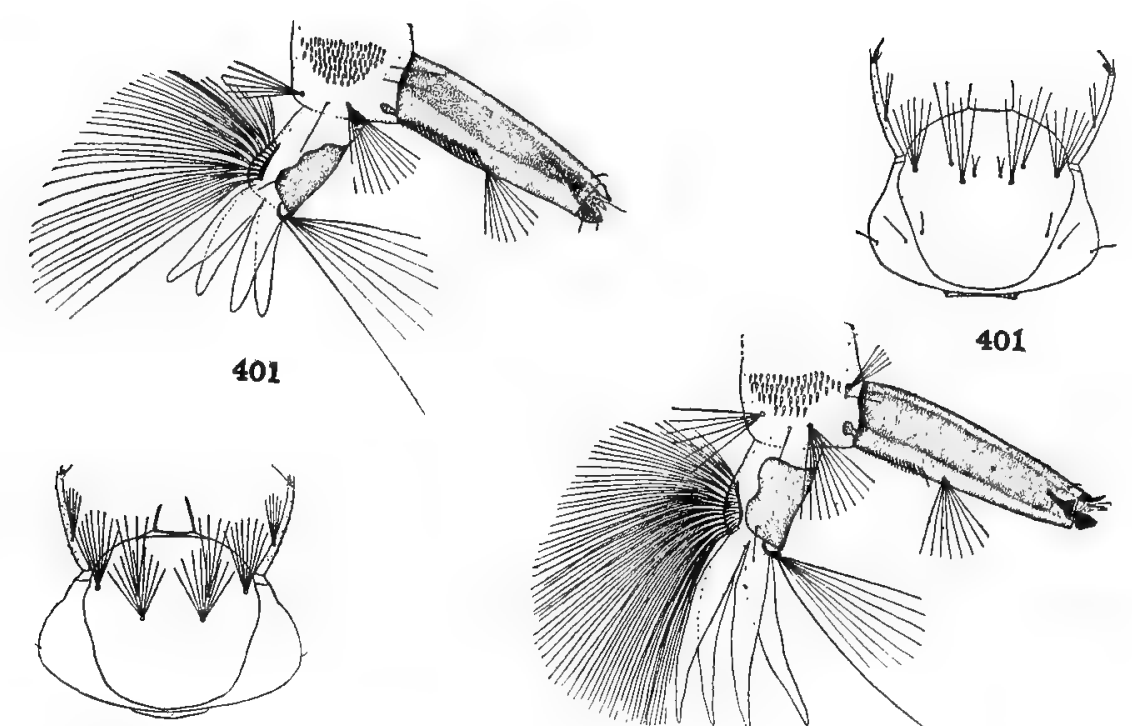

402

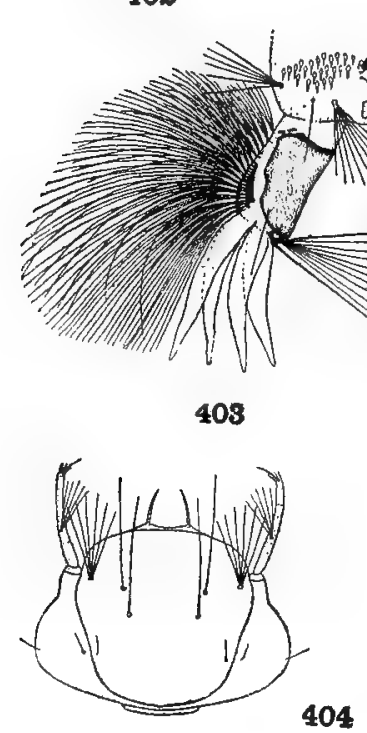

404

DETAILS OF LARV PE.
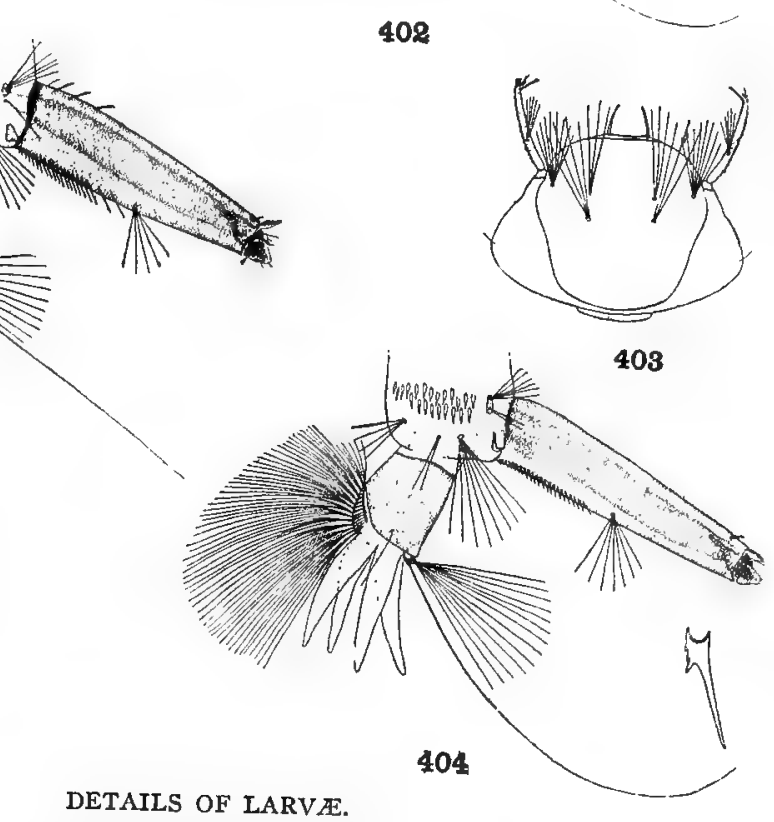

40I. Aëdes fluviatilis Lutz.

402. Aëdes pullatus Coquillett.

403. Aëdes fletcheri Coquillett.

404. Aëdes mitchellæ Dyar. 



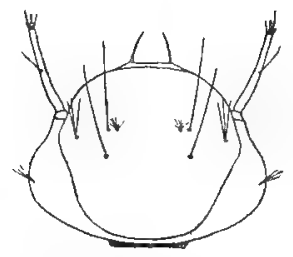

405
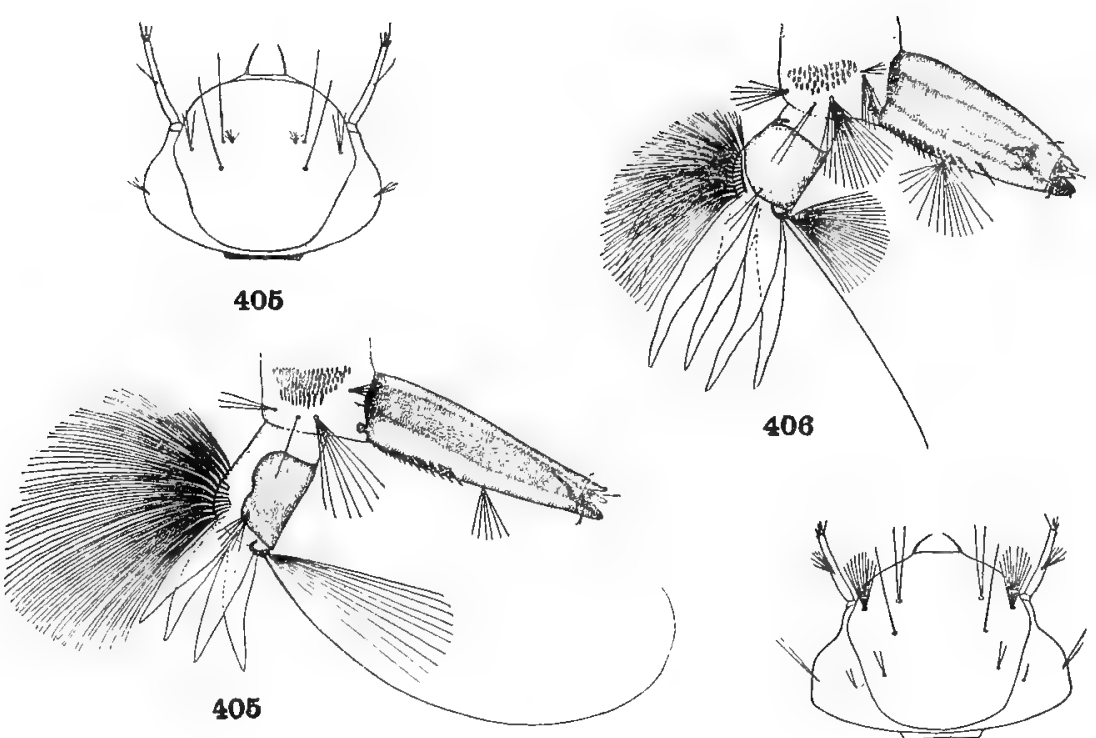

406
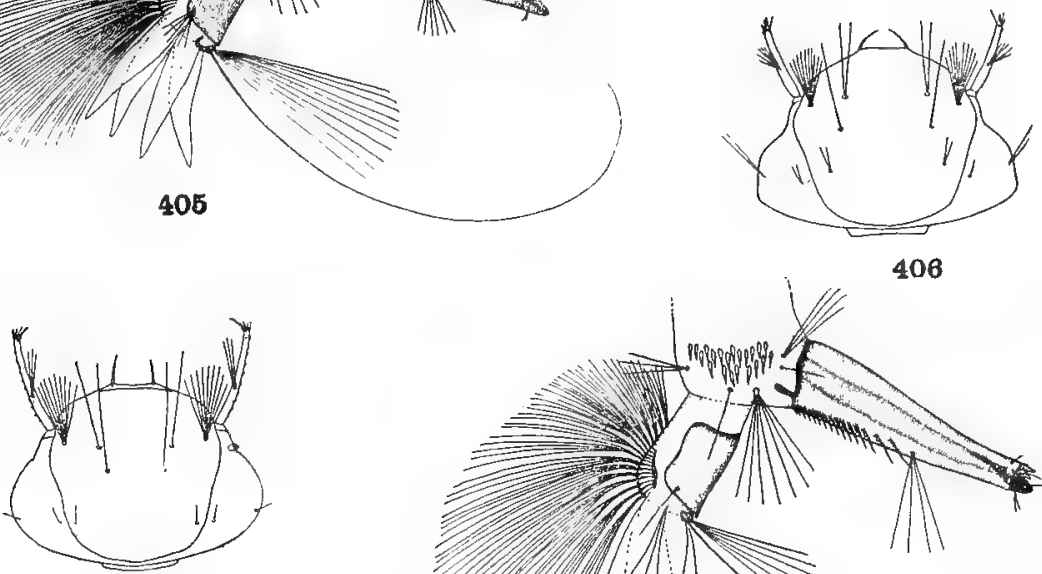

407
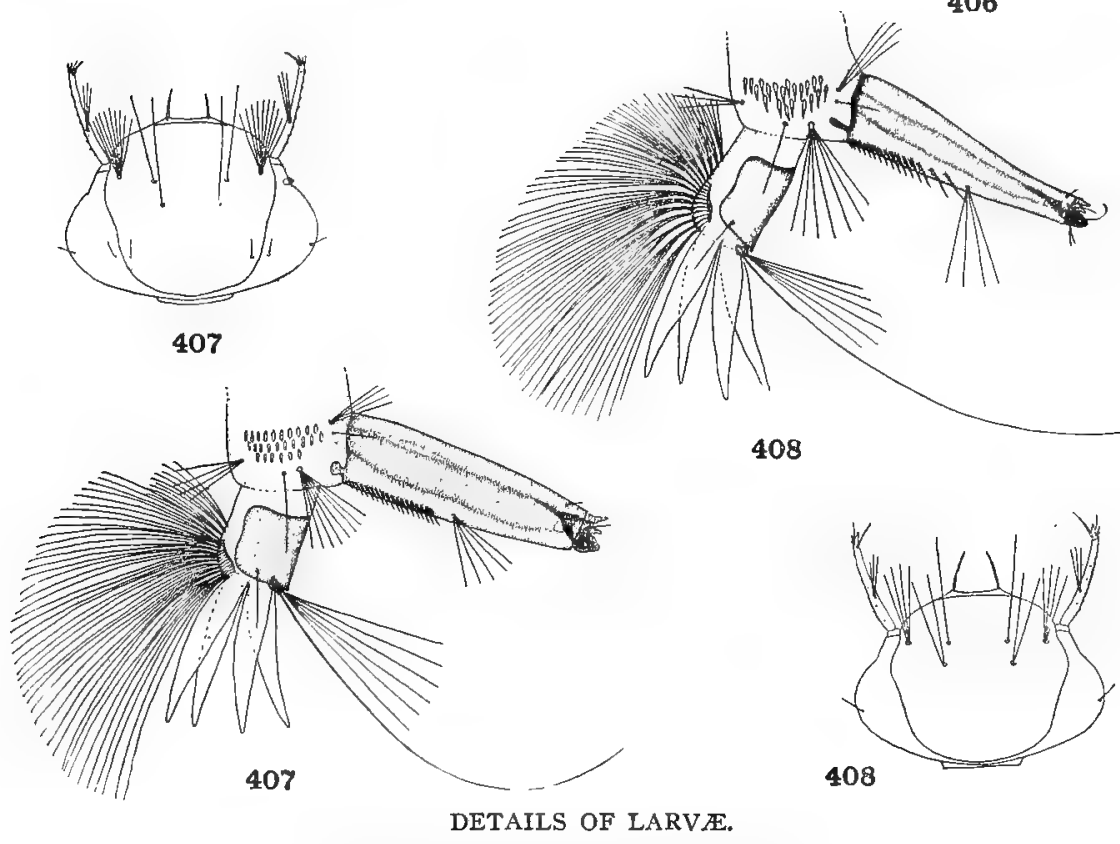

408

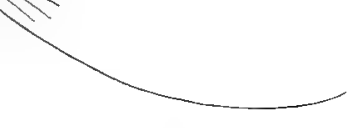

405. Aëdes thorntoni Dyar \& $K n a b$.

406. Aëdes bimaculatus Coquillett.

407. Aëdes lazarensis Felt \& Young.

408. Aëdes sansoni Dyar \& Knab.

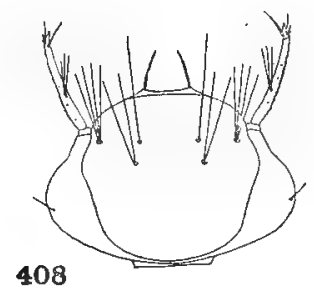




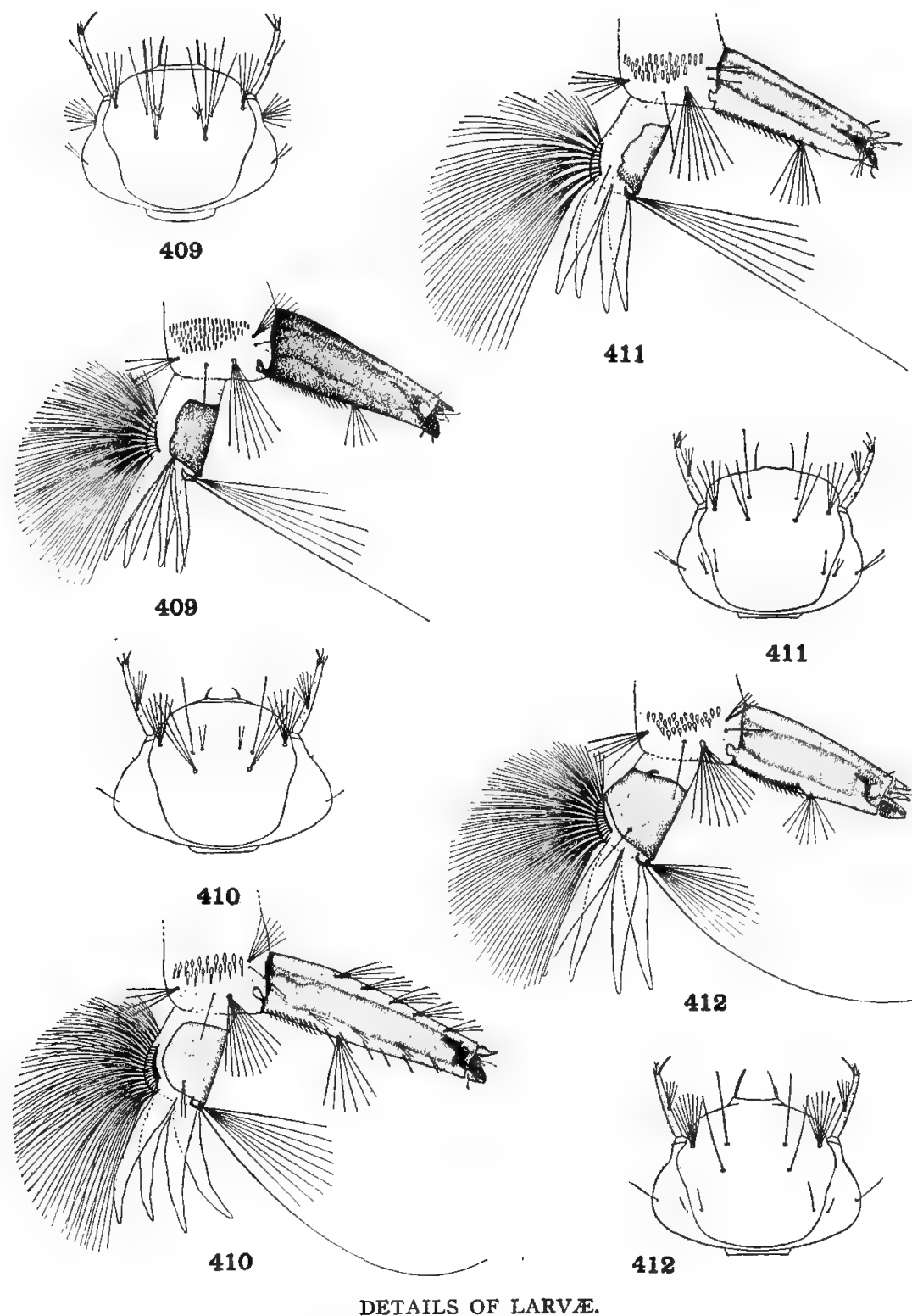

411
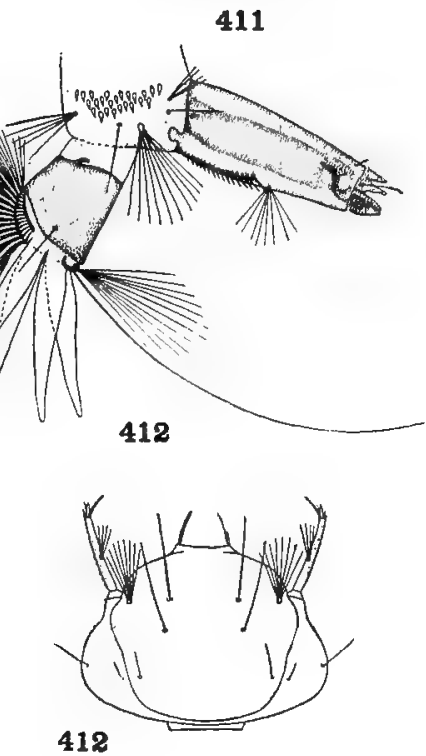

409. Aëdes oswaldi Lutz.

4IO. Aëdes trichurus Dyar.

4II. Aëdes epactius Dyar \& Knab.

412. Aëdes cuneatus Dyar $\mathcal{E} K n a b$. 



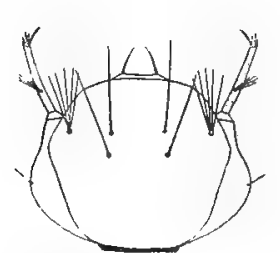

413
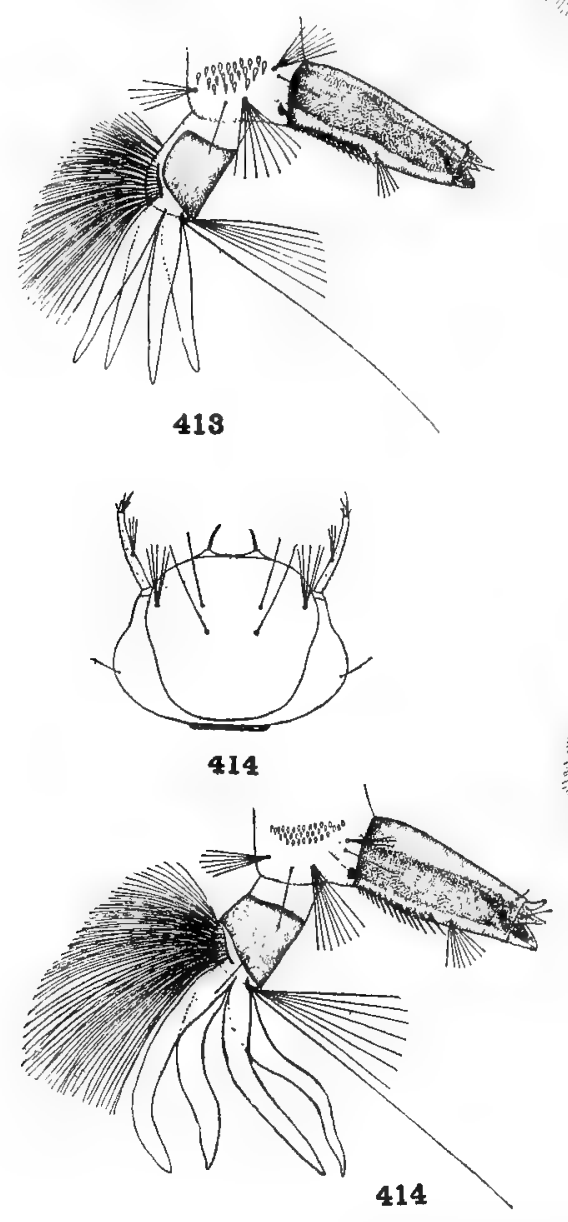

DETAILS OF LARVE.
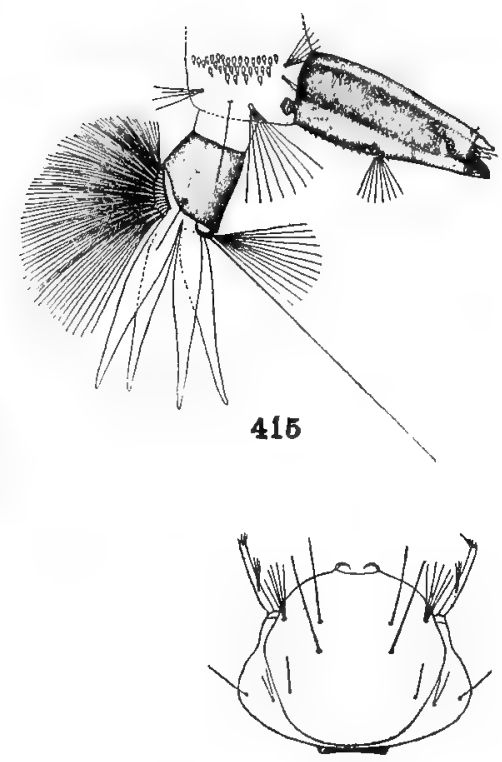

415

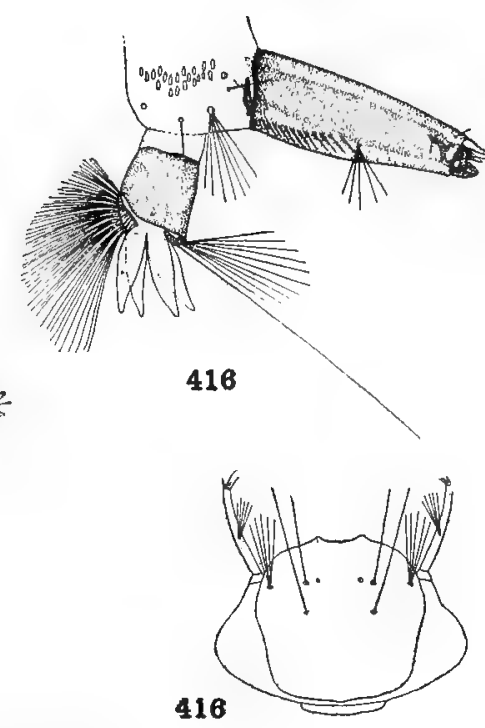

413. Aëdes æstivalis Dyar.

4I4. Aëdes scapularis Rondani.

4I5. Aëdes tortilis Theobald.

4I6. Aëdes bracteatus Coquillett. 



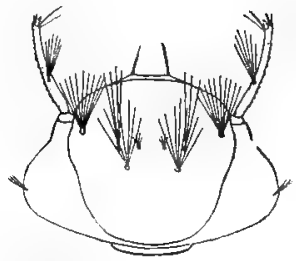

417
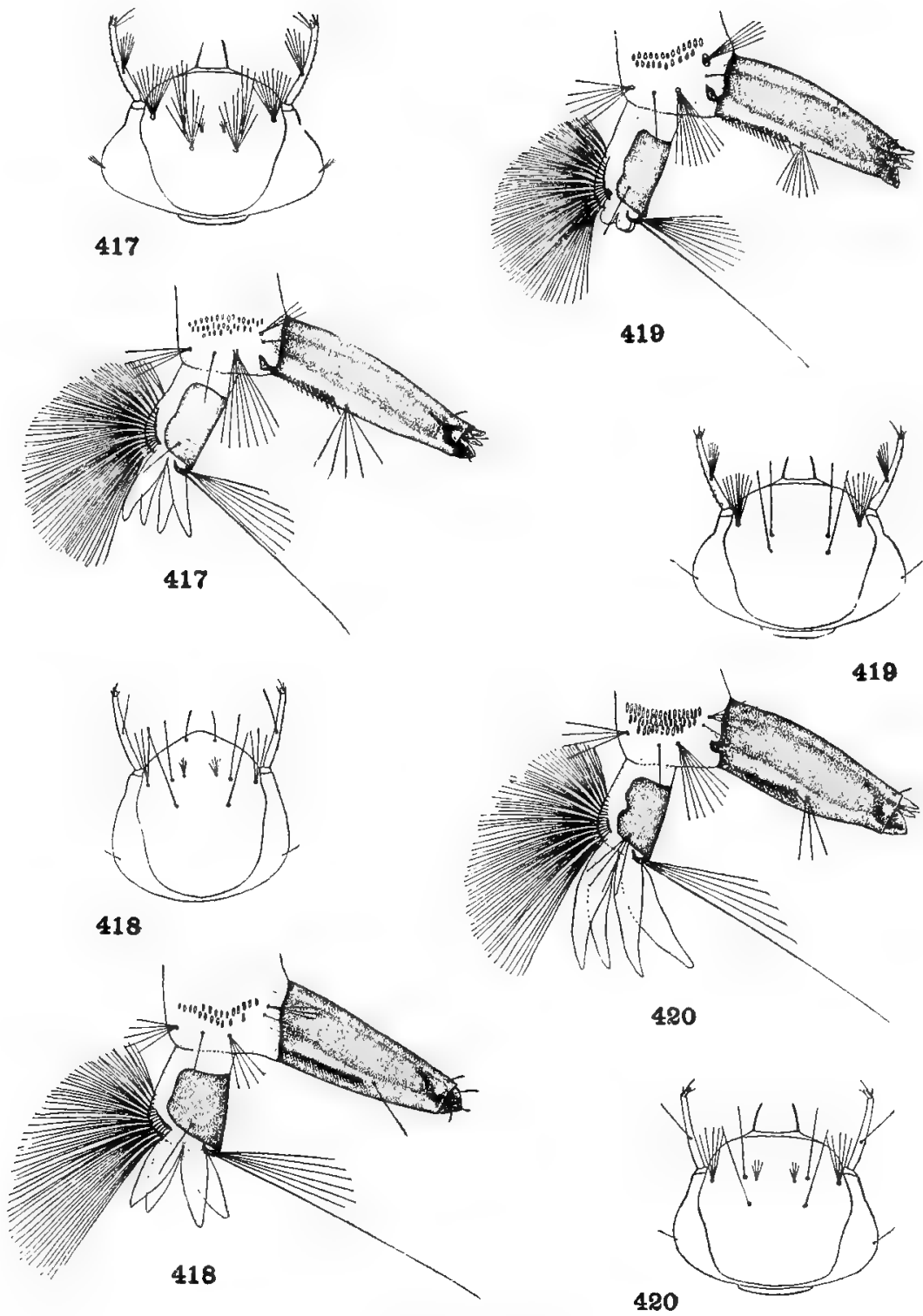

418
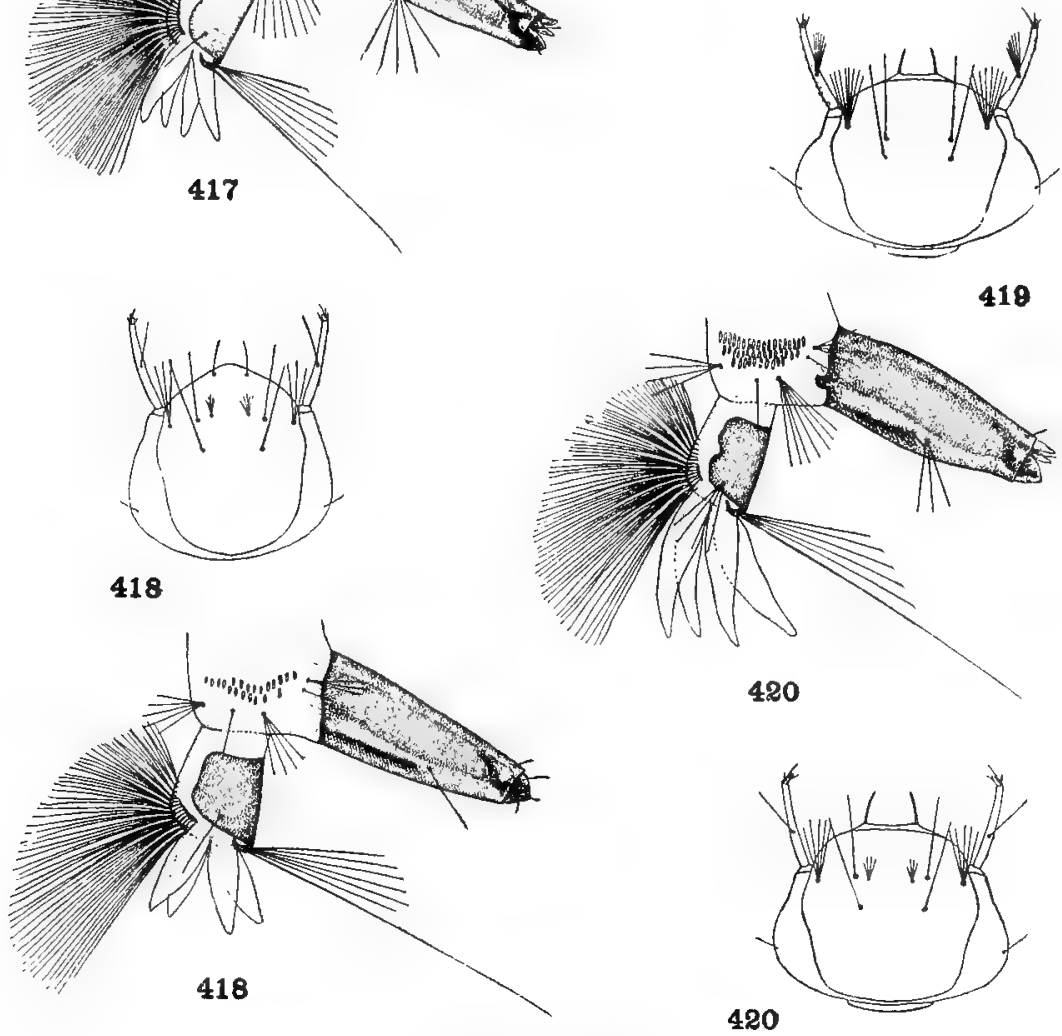

DETAILS OF LARV E.

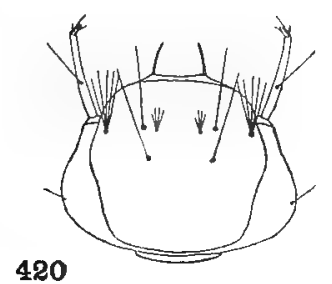

4I7. Aëdes curriei Coquillett.

4I8. Aëdes knabi Coquillett.

419. Aëdes onondagensis quaylei Dyar \& $K n a b$.

420. Aëdes podographicus $D y a r$ \& $K n a b$. 



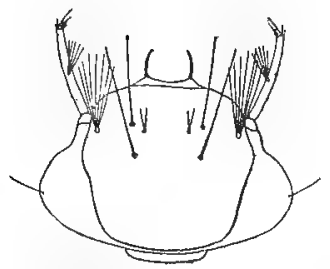

421
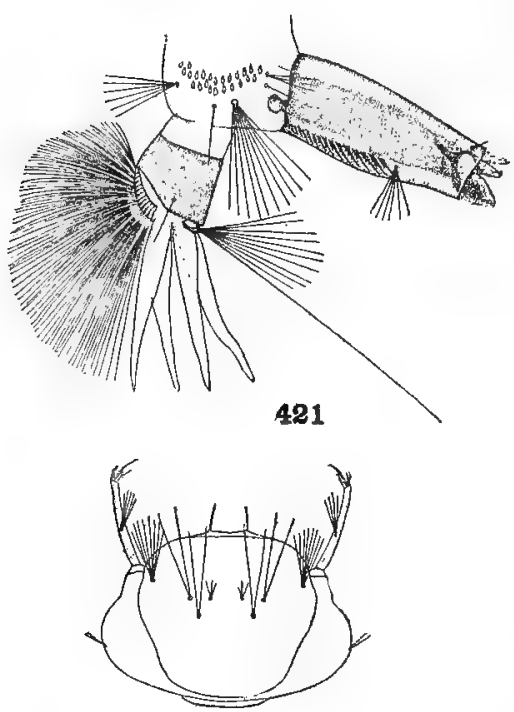

422

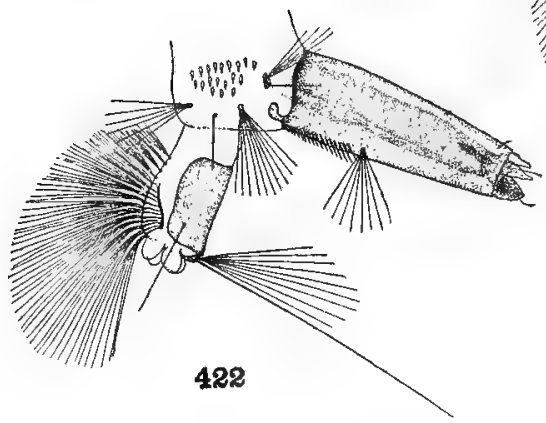

DETAILS OF LARV王.

421. Aëdes euplocamus Dyar \& $K n a b$. 422. Aëdes squamiger Coquillett.

423. Aëdes niger Giles.

424. Aëdes busckii Coquillett.
429

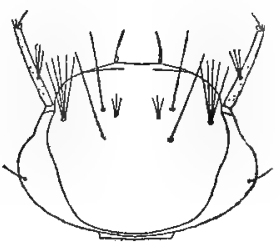

428
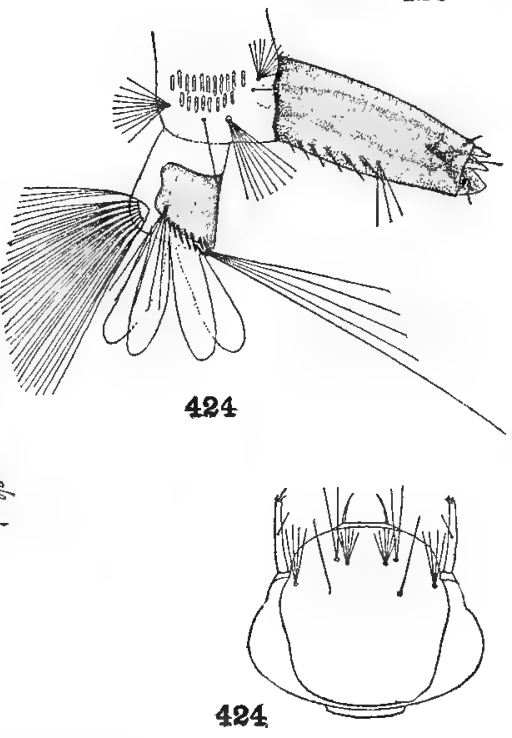



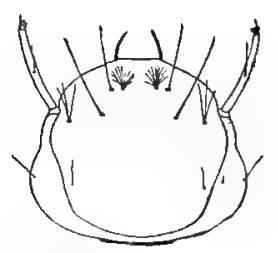

485
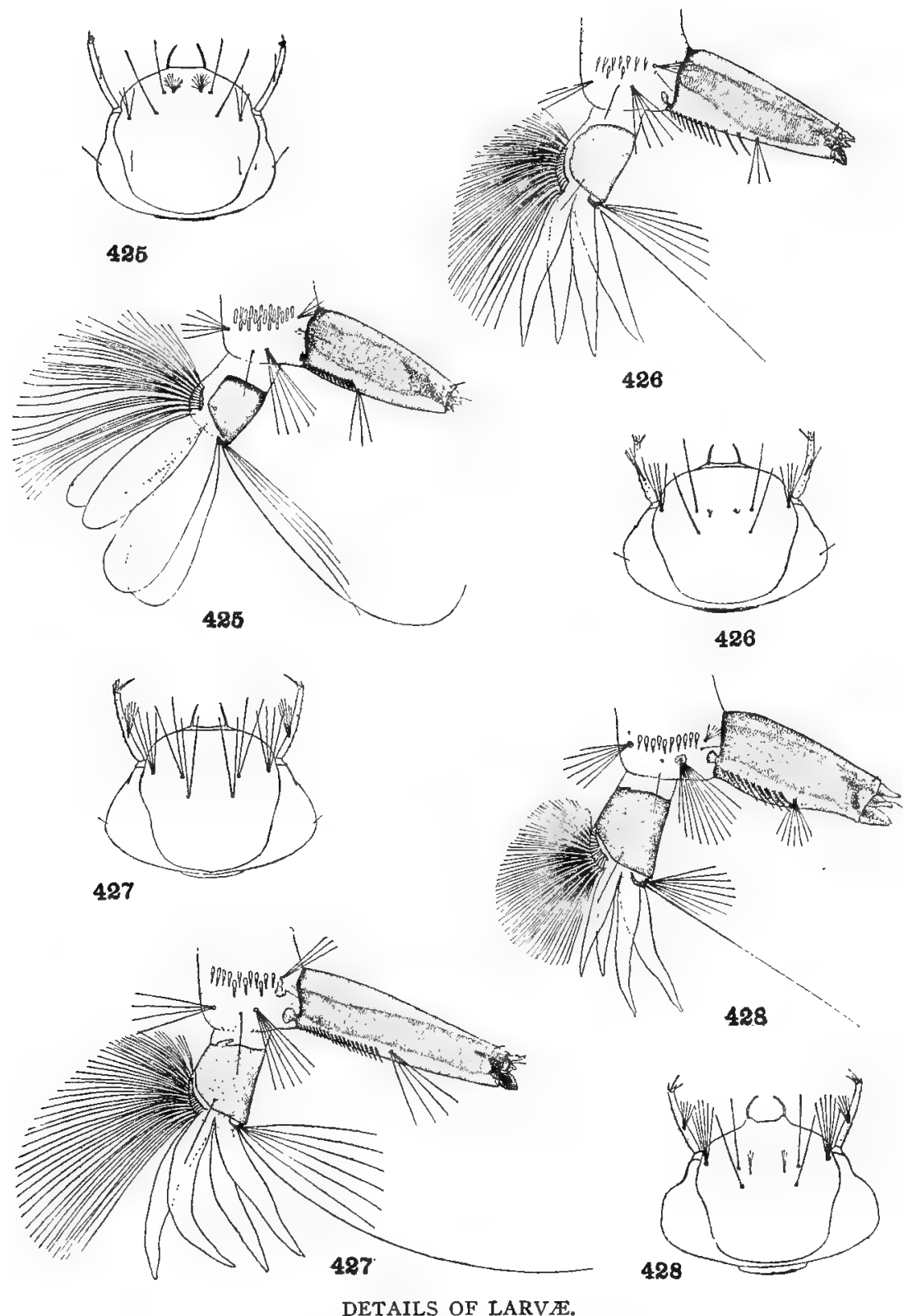

425. Aëdes fulvithorax Lutz.

426. Aëdes spencerii Theobald.

427. Aëdes auroides Felt.

428. Aëdes serratus Theobald.

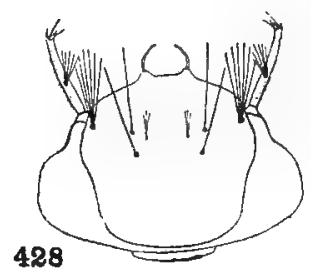




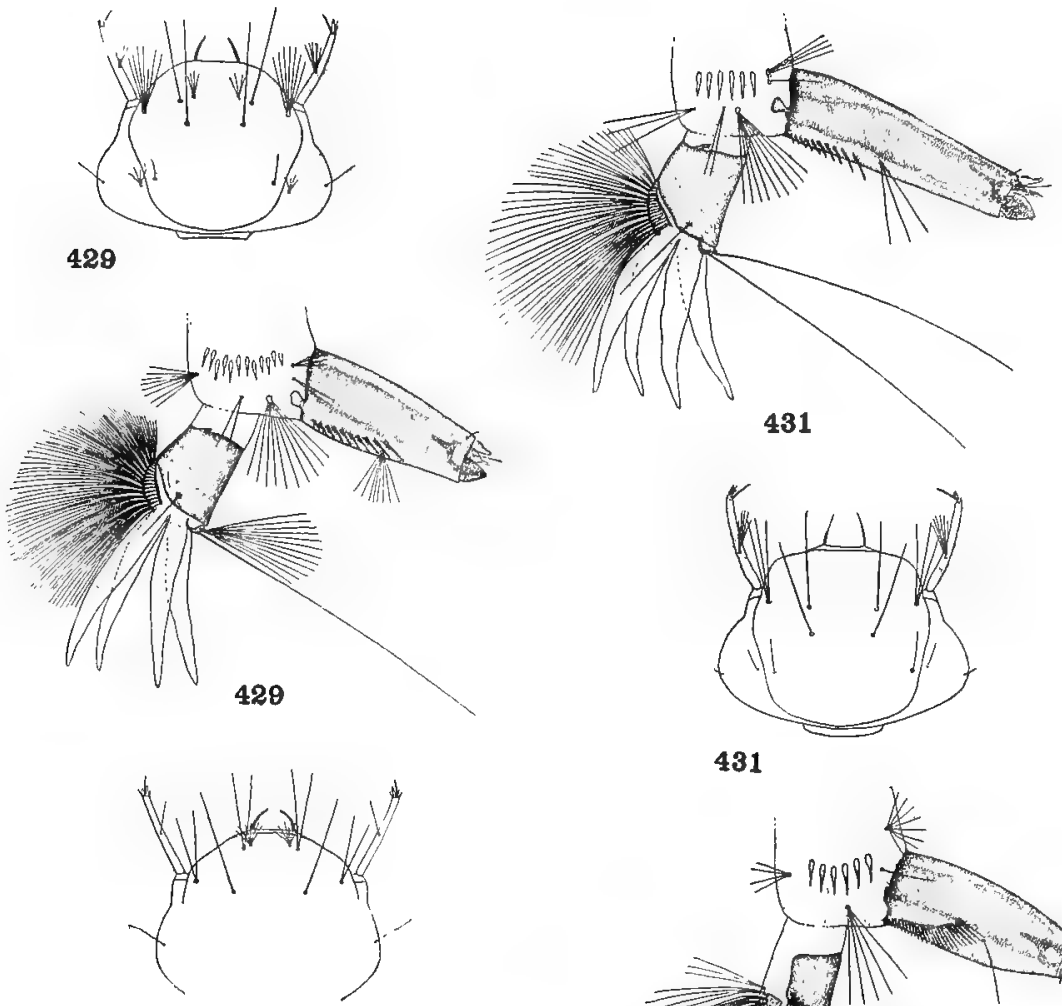

431

430

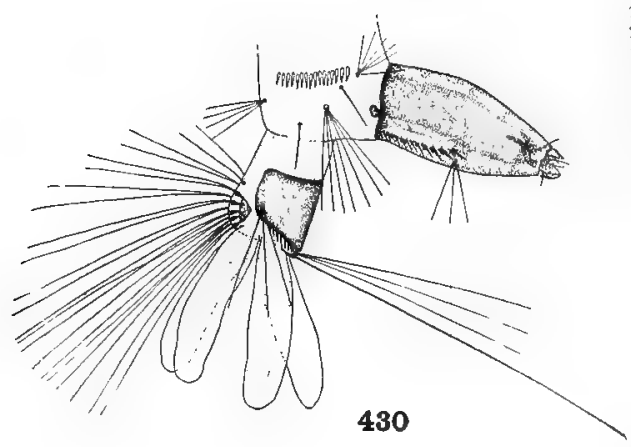

DETAILS OF LARVA.
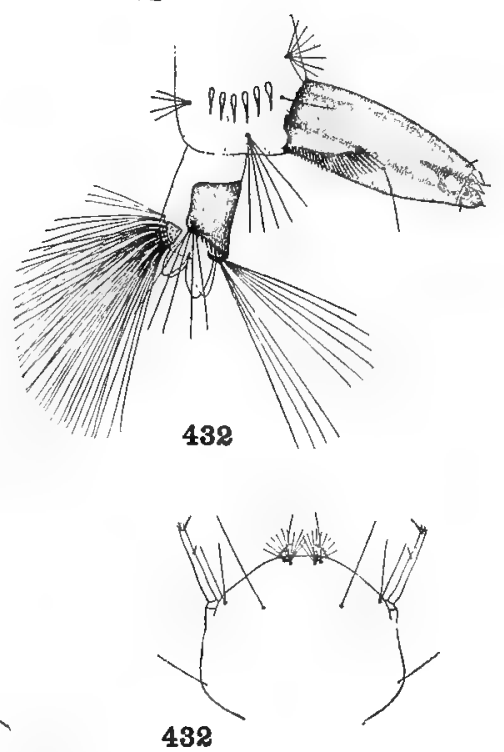

429. Aëdes pertinax Grabham.

430. Aëdes albonotata Coquillett.

43I. Aëdes abserratus Felt \& Young.

432. Aëdes mediovittata Coquillett. 


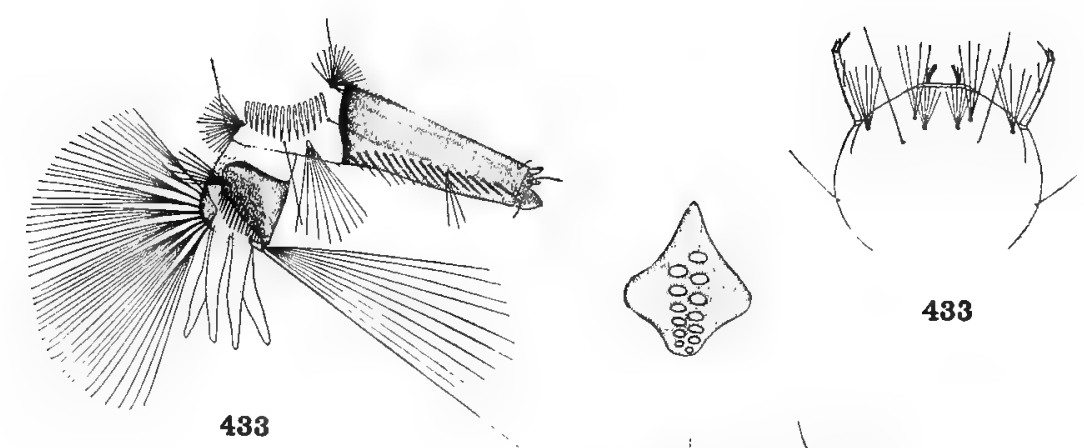

433

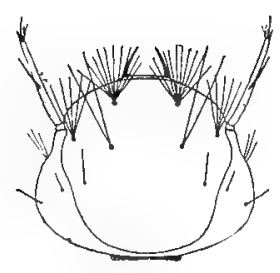

434
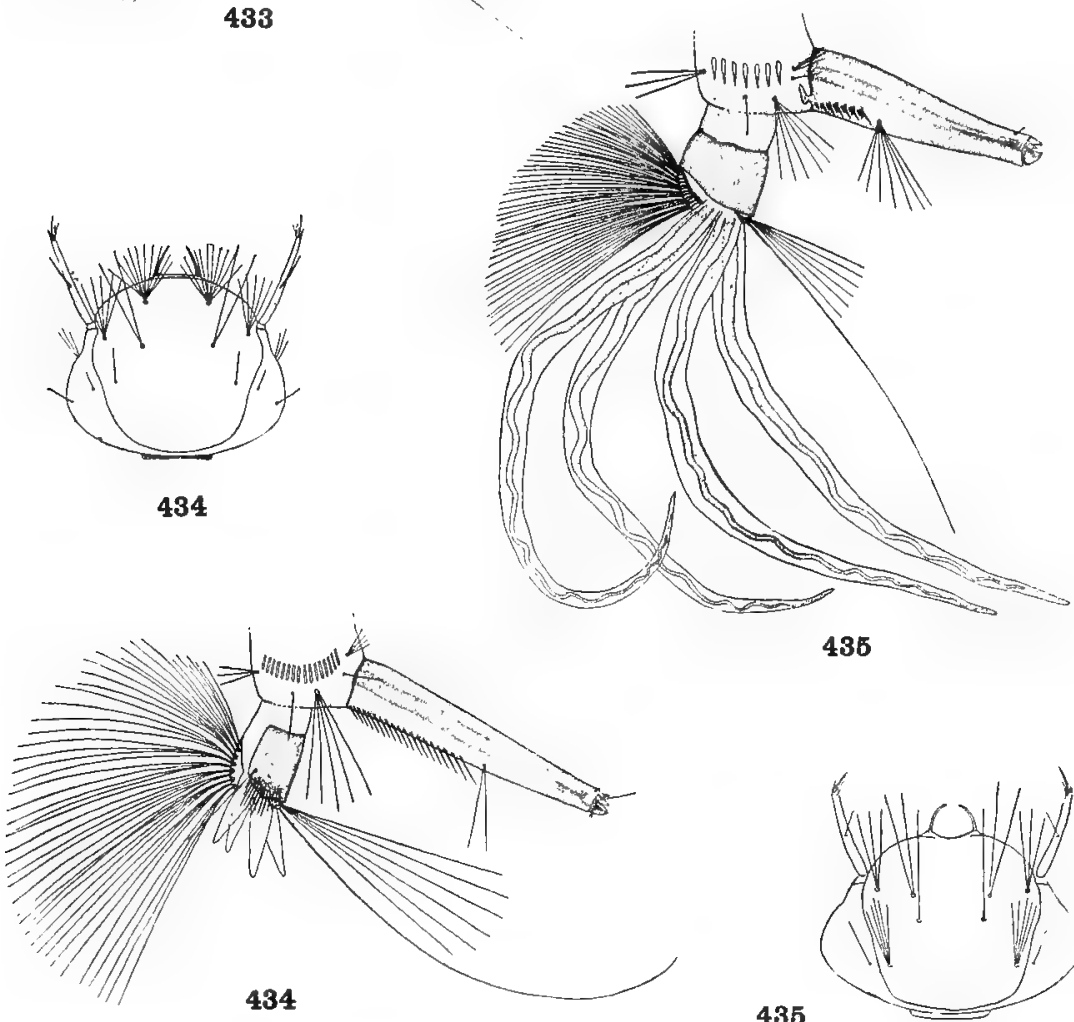

435

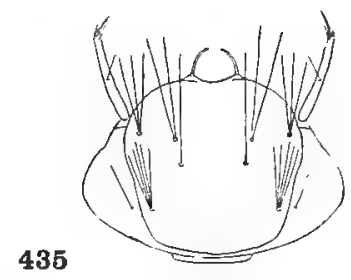

DETAILS OF LARVAE.

433. Aëdes walkeri Theobald.

434. Aëdes aureostriata Grabham.

435. Aëdes dupreei Coquillett. 


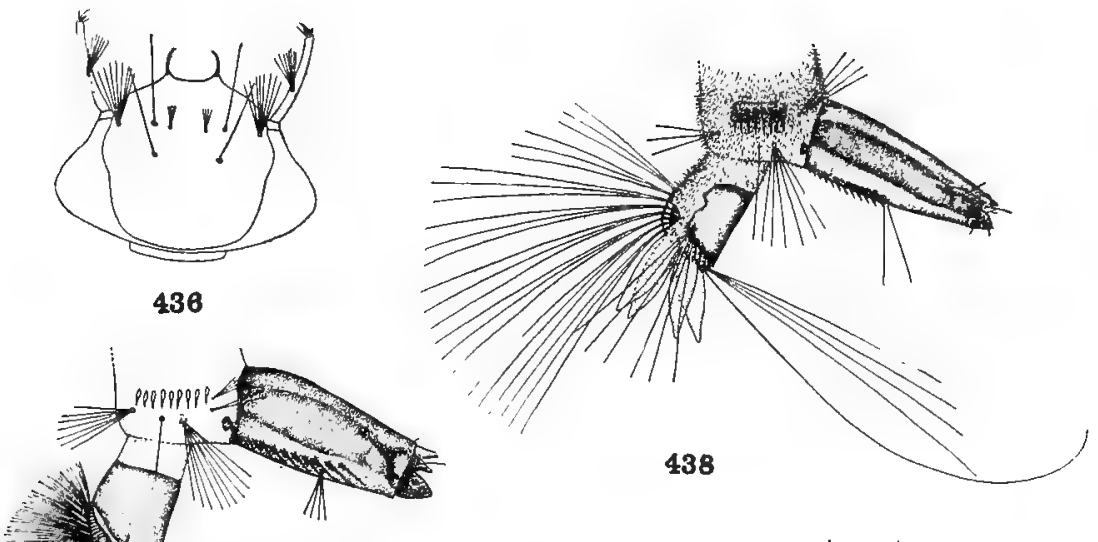

438

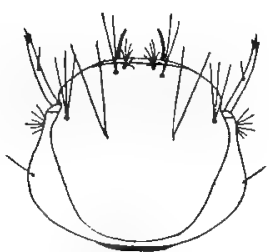

436

438

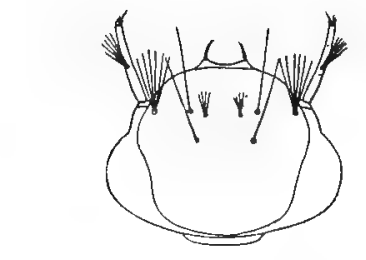

437
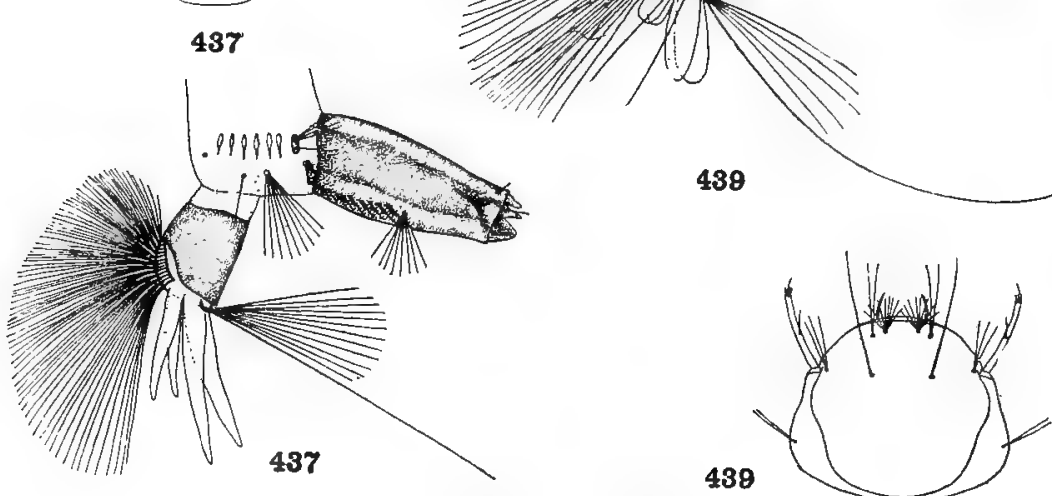

DETAILS OF LARVF.

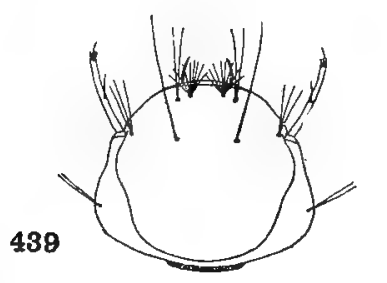

436. Aëdes tormentor Dyar \& Knab.

437. Aëdes atlanticus $D y a r$ \& $K n a b$.

438. Stegoconops capricornii Lutz.

439. Stegoconops albomaculatus Theobald. 



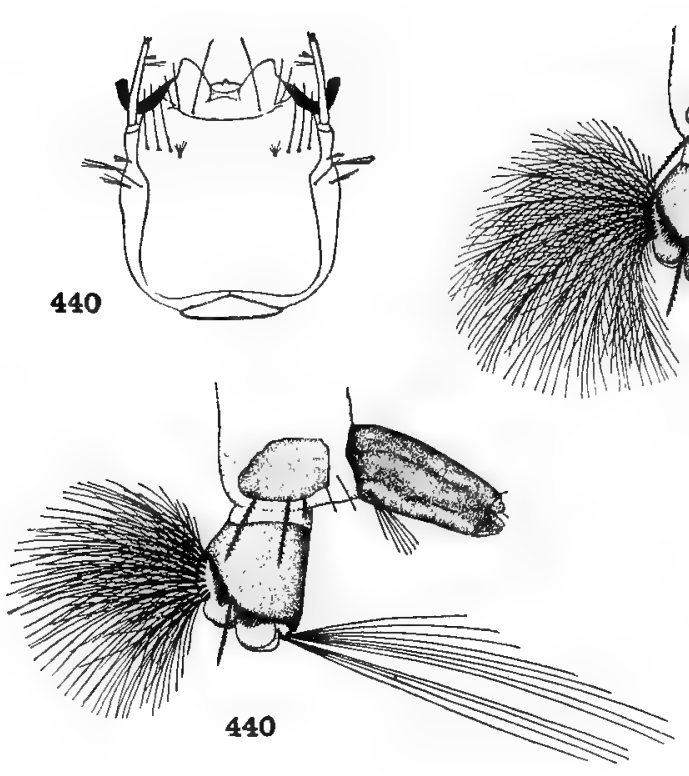

\section{1}
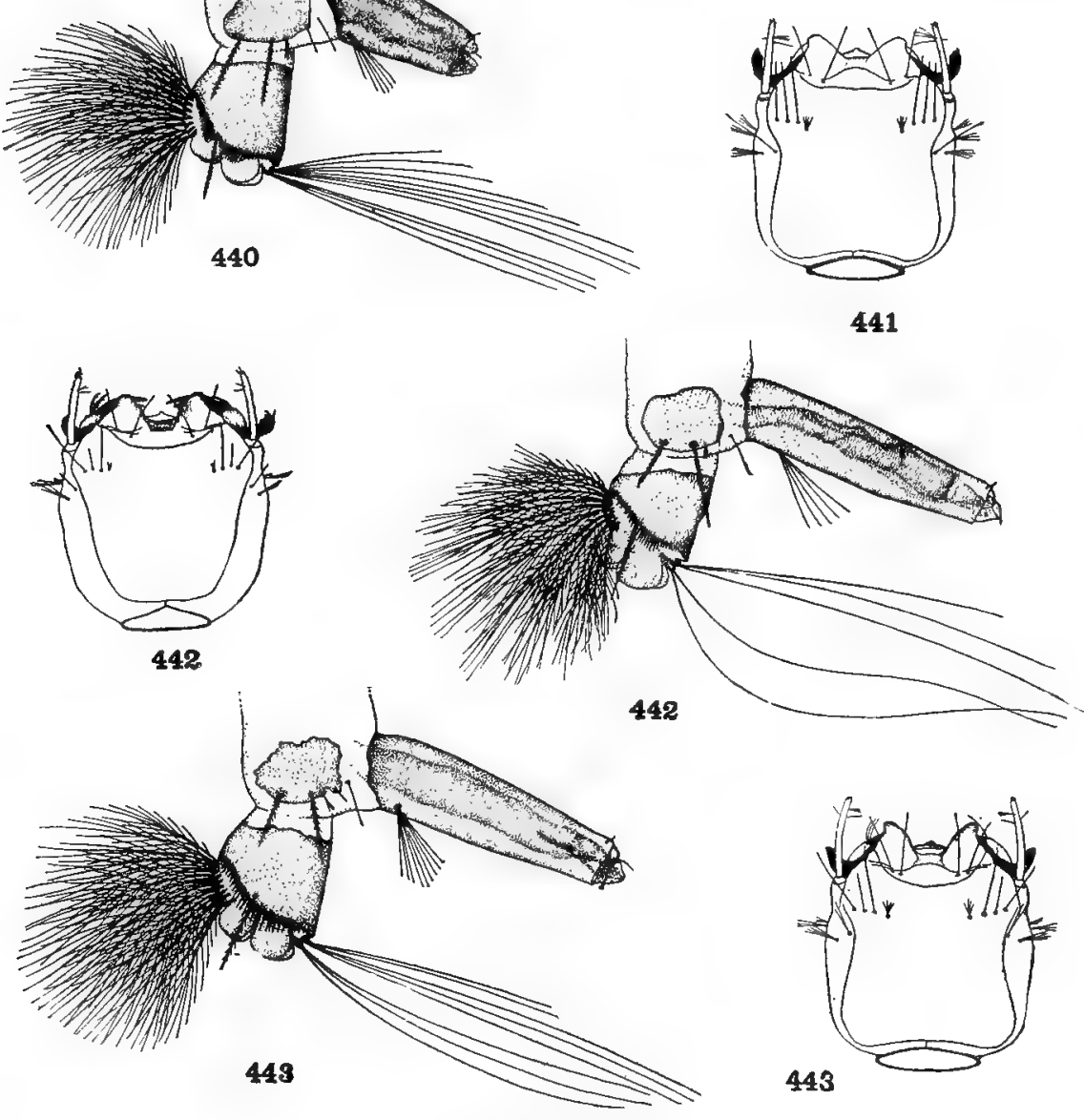

DETAILS OF LARVA.

440. Megarhinus portoricensis von Roeder.

44I. Megarhinus trinidadensis Dyar \& $K n a b$.

442. Megarhinus superbus Dyar \& $K n a b$.

443. Megarhinus guadeloupensis Dyar \& $K n a b$. 


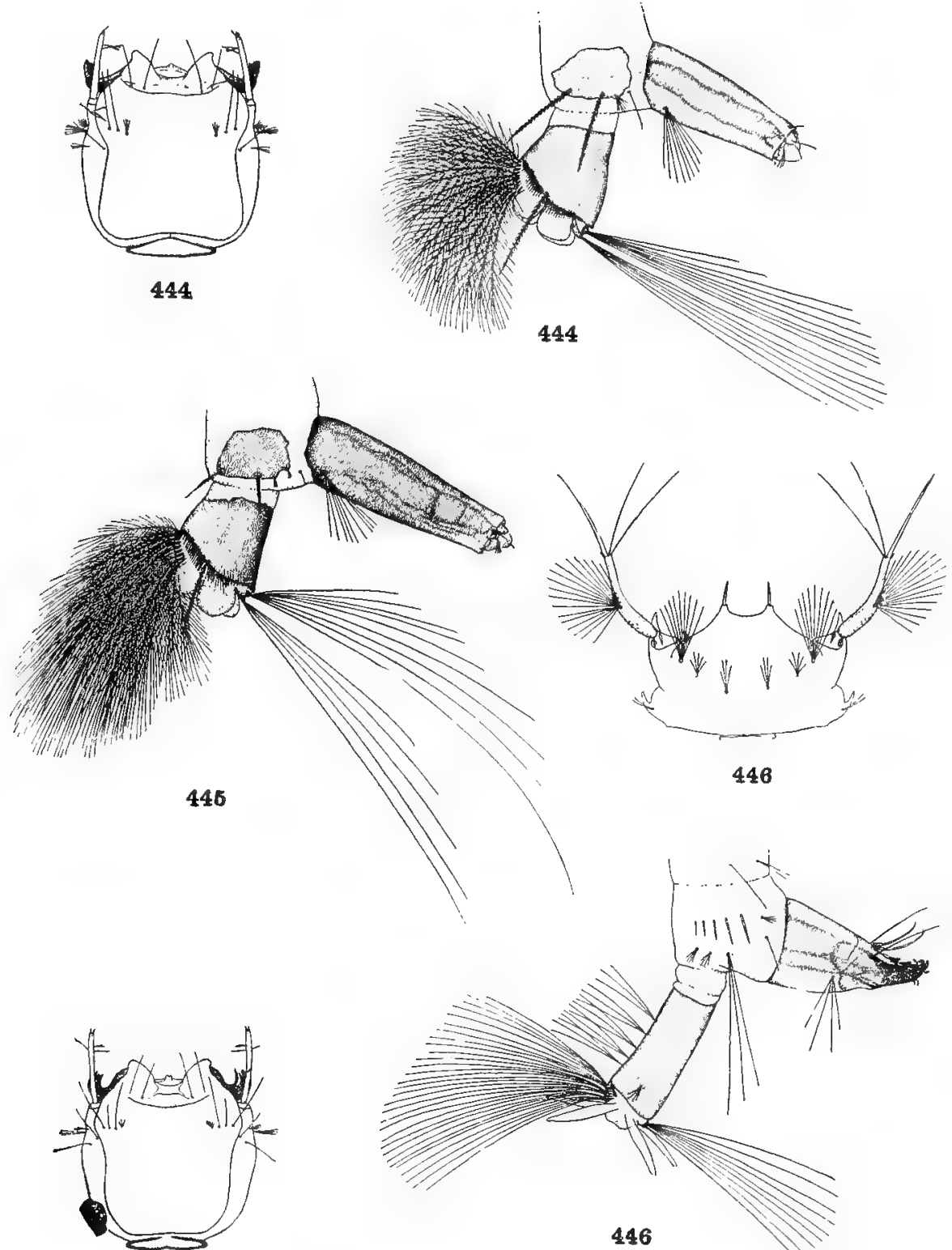

445

DETAILS OF LARV $邓$.

444. Megarhinus moctezuma $D$ yar \& Knab. 445. Megarhinus hypoptes Knab.

446. Mansonia titillans Walker. 


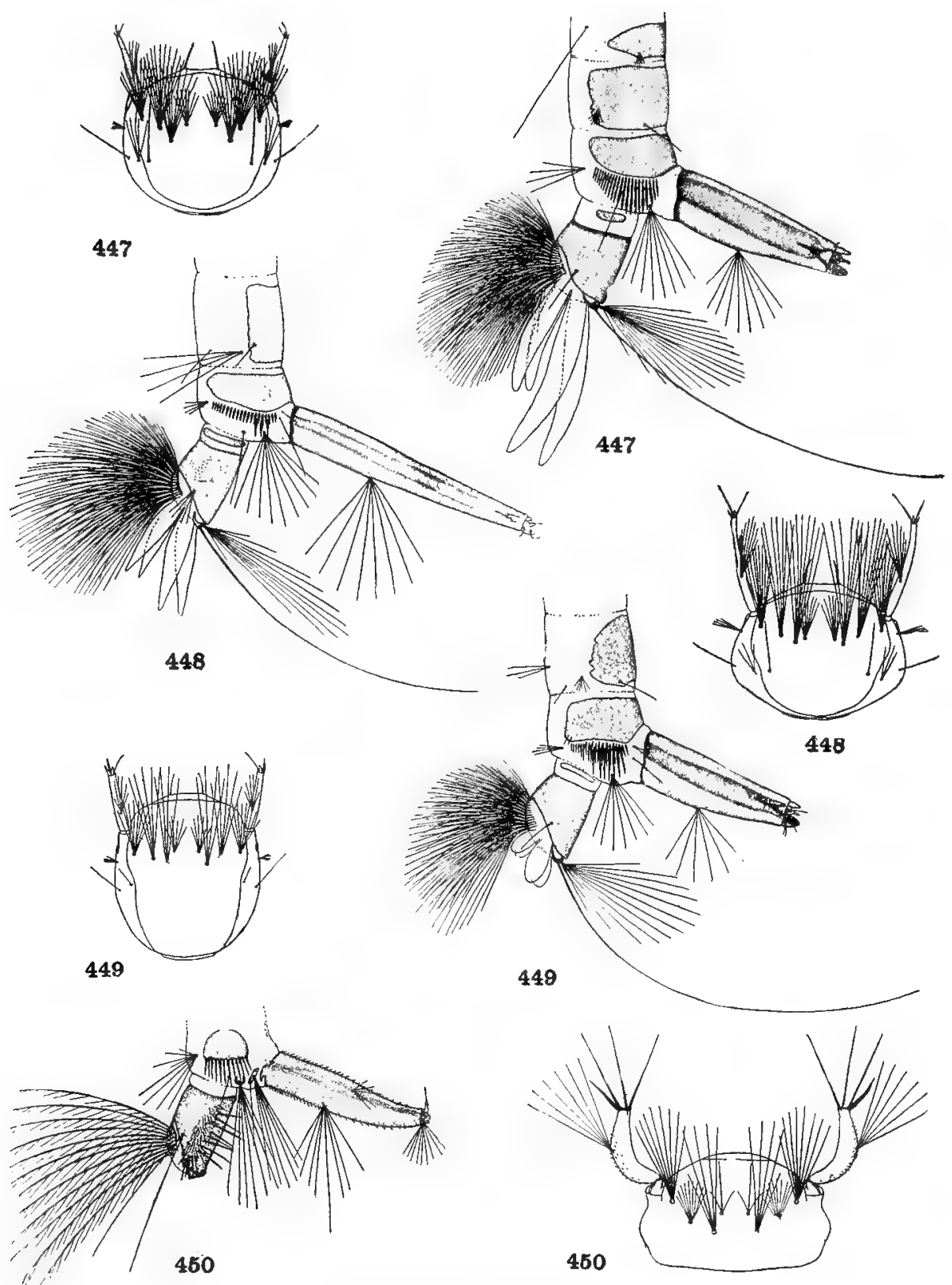

DETAILS OF LARVA.

447. Bancroftia fascipes Caquillett.

448. Bancroftia phyllozoa Dyar \& Knab.

449. Bancroftia waverleyi Grabham.

450. Aëdeomyia squamjpennis Arribalzaga. 


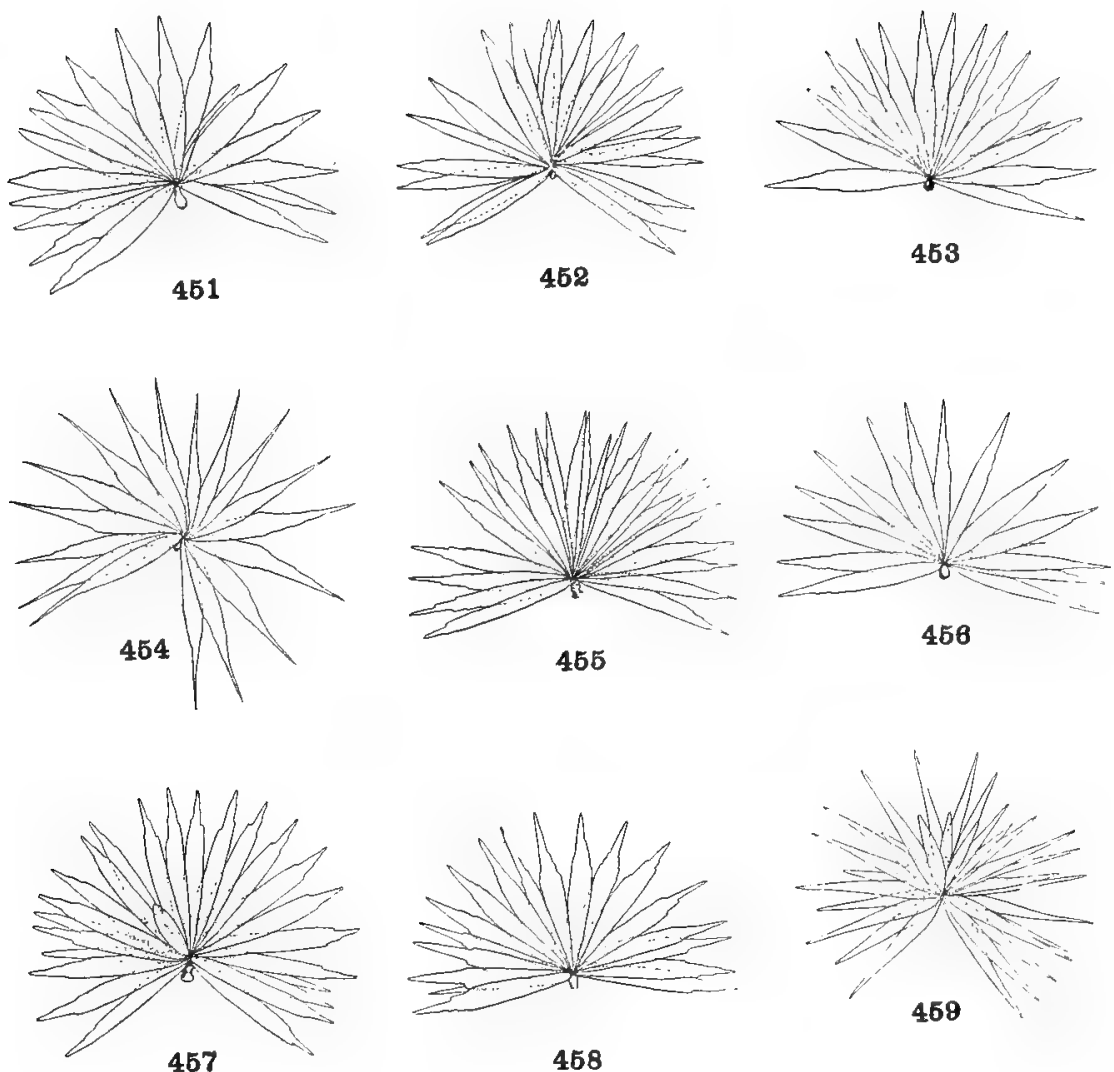

457

458

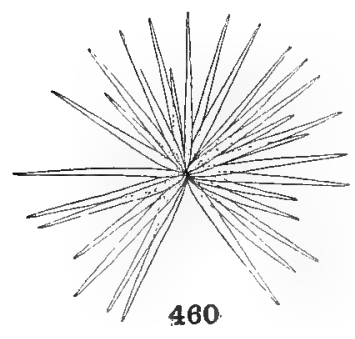

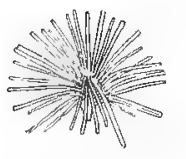

461

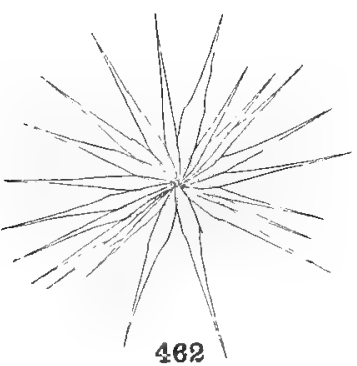

LARVAL FLOAT-HAIRS.
45I. Anopheles punctipennis Say.

452. Anopheles crucians Wiedemann.

453. Anopheles quadrimaculatus Say.

454. Anopheles pseudopunctipennis Theob.

455. Anopheles grabhamii Theobald.

456. Anopheles strigimacula Dyar \& Knab.

457. Anopheles eiseni Coquillett.
458. Anopheles malefactor Dyar \& Knab. 459. Anopheles argyritaris Robineau-Desvoidy.

460. Anopheles albimanus Wiedemann and A. tarsimaculata Goeldi.

46r. Anopheles neivai $H ., D$. \& $K$. 462. Colodiazesis barberi Coquillett. 


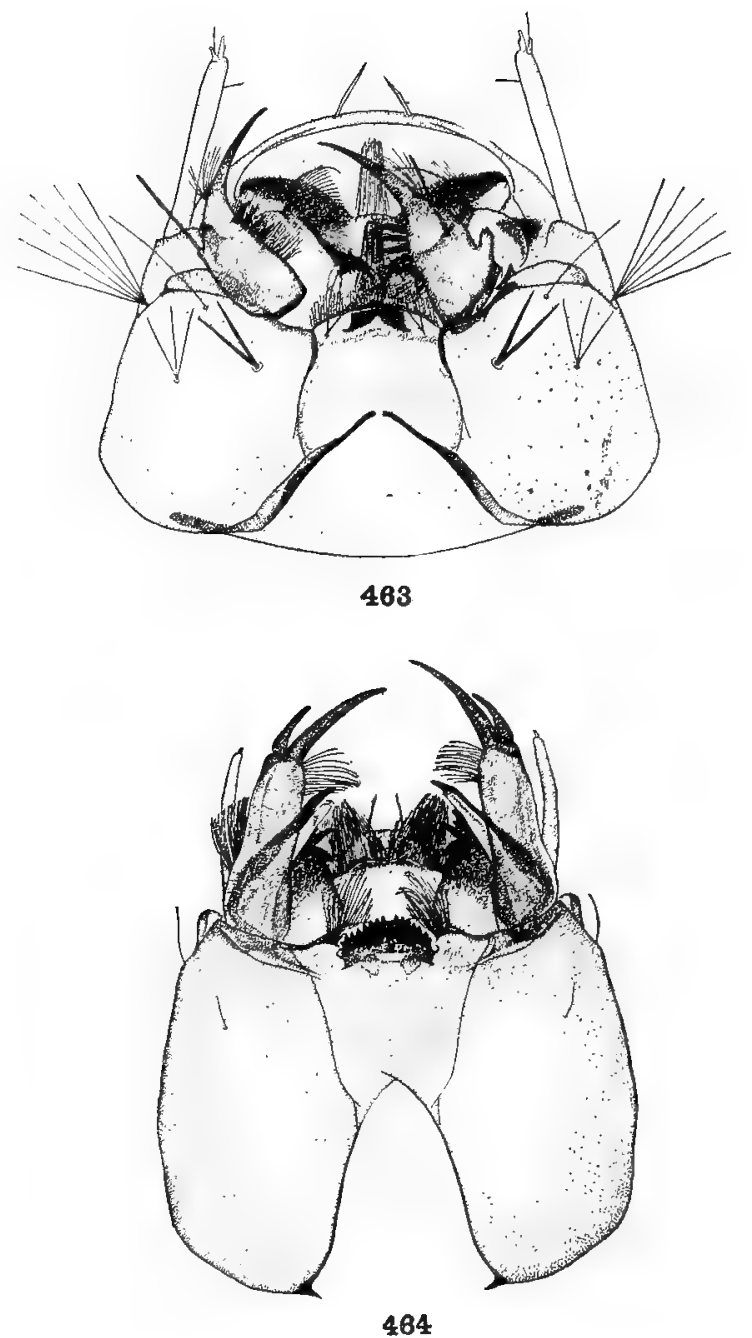

HEAD OF LARVA, VENTRAL VIEW.

463. Sabethinus undosus Coquillett. 464. Lesticocampa rapax Dyar \& Knab. 


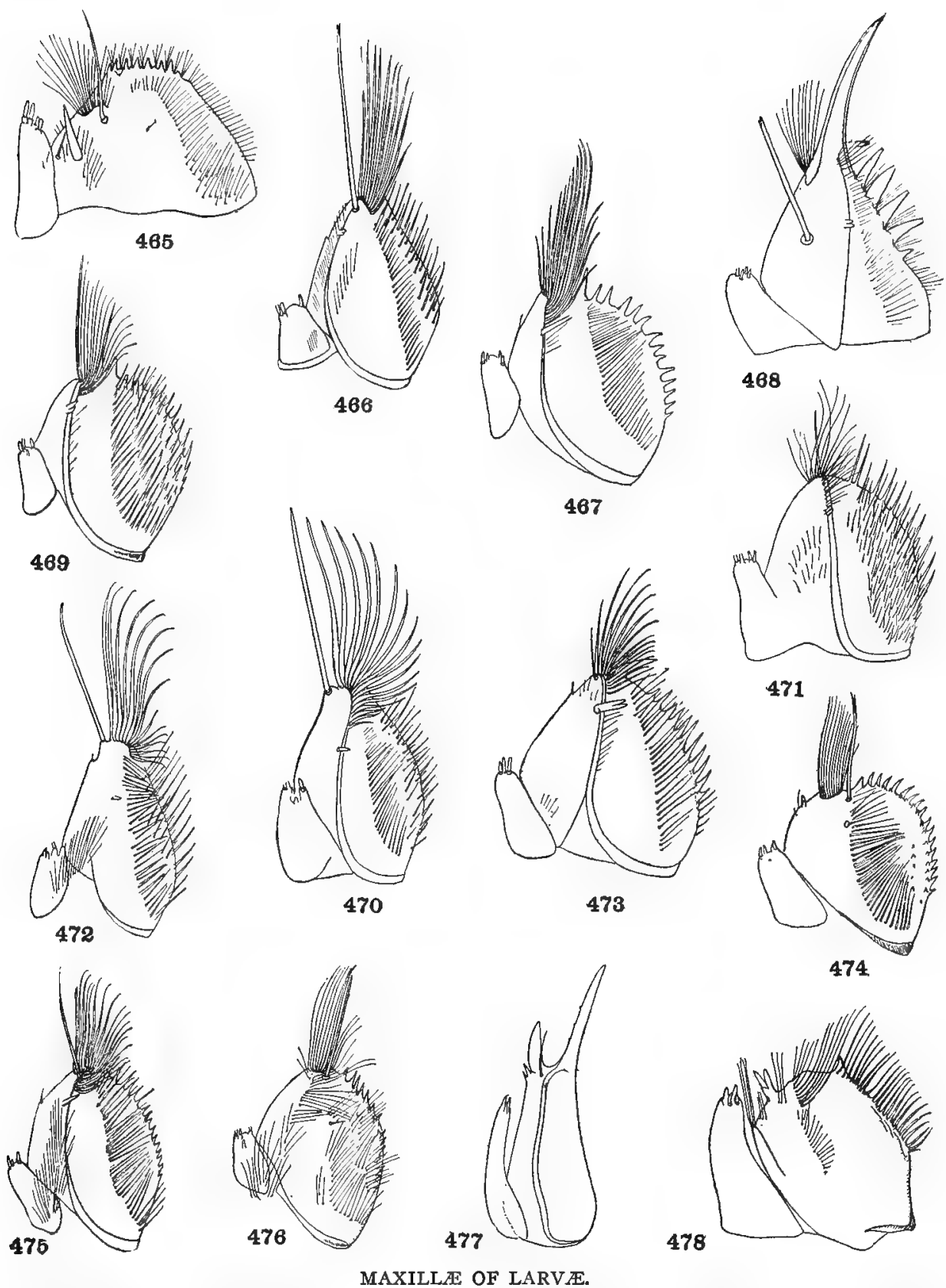

474

MAXILLA OF LARVÆ.

465. Limatus durhamii Theobald.

466. Wyeomyia trinidadensis Theobald.

467. Wyeomyia aporonoma $D y a r$ \& $K n a b$.

468. Sabethinus undosus Coquillett.

469. Wyeomyia sororcula Dyar \& Knab.

470. Wyeomyia mitchellii Theobald.

471. Wyeomyia smithii Coquillett.
472. Wyeomyia autocratica Dyar \& $K n a b$. 473. Wyeomyia pseudopecten Dyar \& Knab. 474. Wyeomyia bromeliarum Dyar \& Knab. 475. Wyeomyia telestica Dyar \& $K n a b$. 476. Wyeomyia hemisagnosta $D$ yar \& $K n a b$. 477. Lesticocampa rapax Dyar \& $K n a b$. 478. Joblotia digitatus Rondani. 


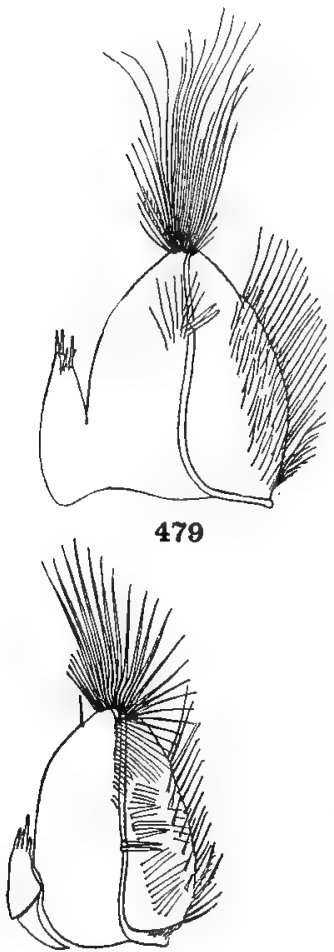

483

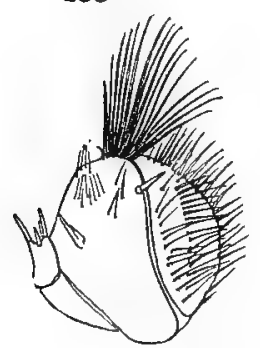

487

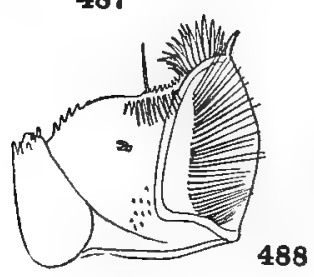

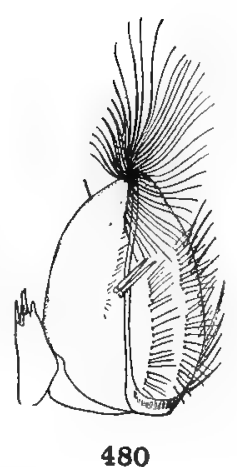

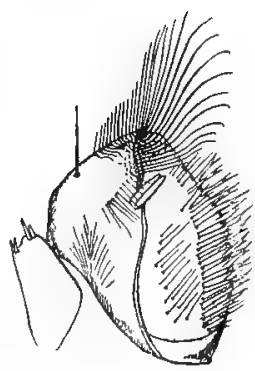

481

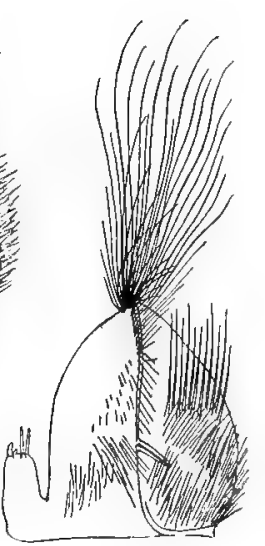

482
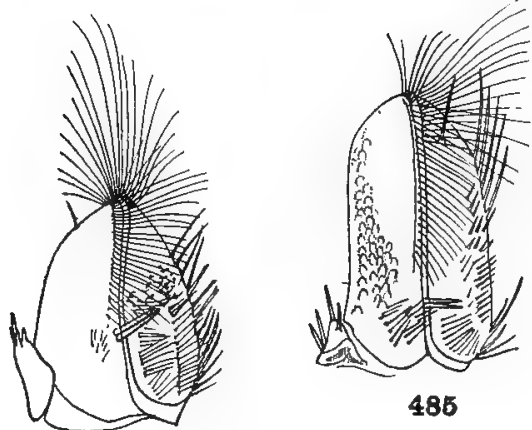

485

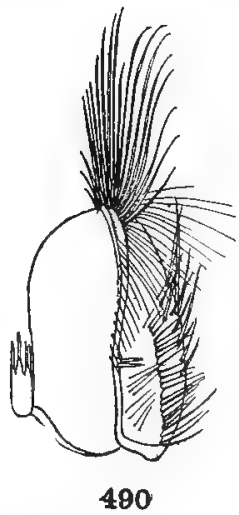

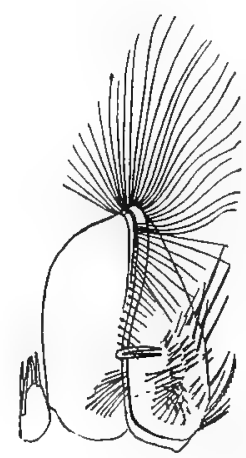

486

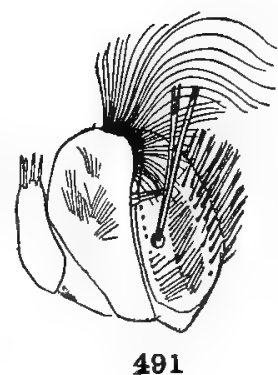

MAXILLE OF LARV无.

479. Deinocerites cancer Theobald.

480. Culex mortificator Dyar \& Knab.

481. Culex corniger Theobald.

482. Culex dyari Coquillett.

483. Culex carmodyz Dyar \& Knab.

484. Culex factor Dyar \& $K n a b$.
485. Culex caudelli Dyar \& Knab. 486. Culex mutator Dyar \& Knab. 487. Culex pleuristriatus Theobald. 488. Lutzia bigoti Bellardi. 489. Culex rejector Dyar \& Knab. 490. Culex inimitabilis Dyar \& Knab. 49I. Carrollia urichii Coquillett. 



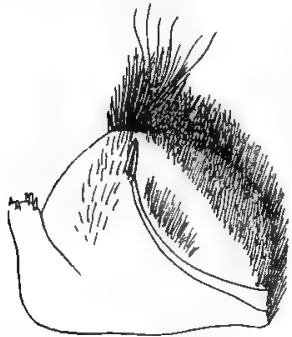

492

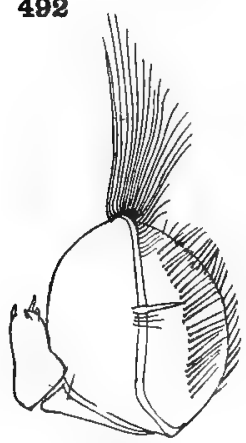

496

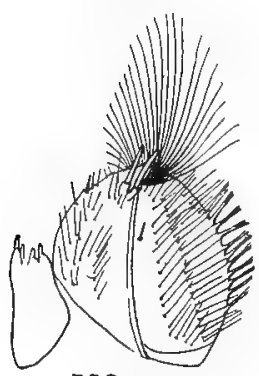

500

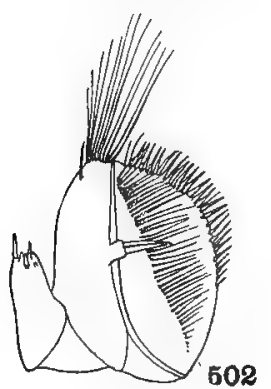

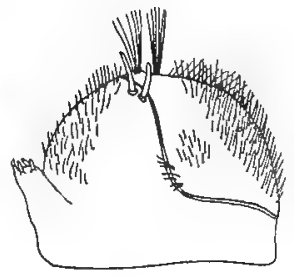

493

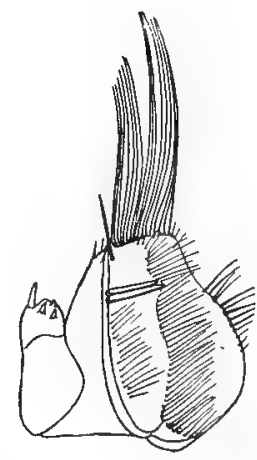

497

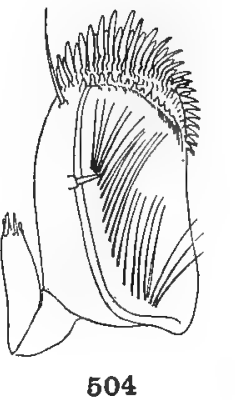

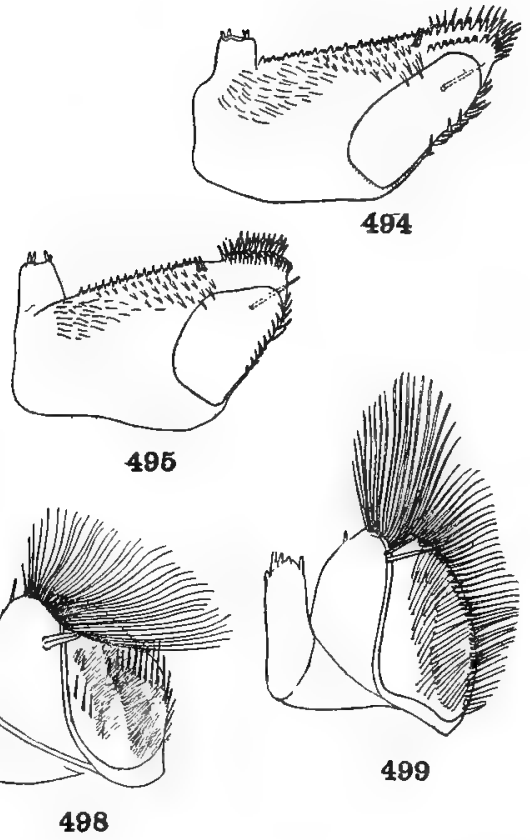

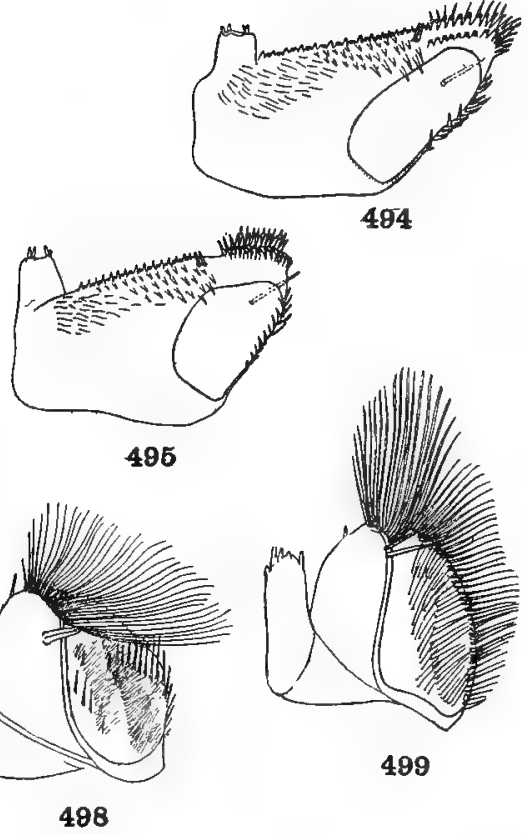

484
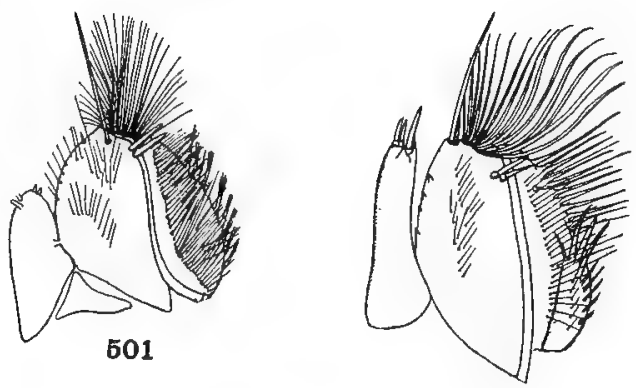

608
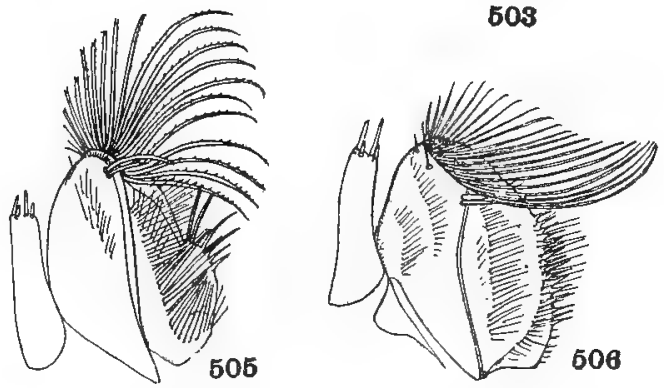

MAXILL正 OF LARVA.

492. Culiseta incidens Thomson.

493. Culiseta inornatus $W$ illiston.

494. Psorophora ciliata Fabricius.

495. Psorophora howardii Coquillett.

496. Psorophora signipennis Coquillett.

497. Aëdes cantator Coquillett.

498. Aëdes lazarensis Felt \& Young.
499. Aëdes trivittatus Coquillett.

500. Aëdes euplocamus Dyar \& Knab.

501. Aëdes atlanticus $D$ yar $\mathcal{E} K n a b$.

502. Aëdes podographicus Dyar \& Knab.

503. Aēdes mediovittata Coquillett.

504. Aëdes knabi Coquillett.

505. Aëdes albonotata Coquillett. 506. Stegoconops lucifer $H$., $D$. \& $K$. 


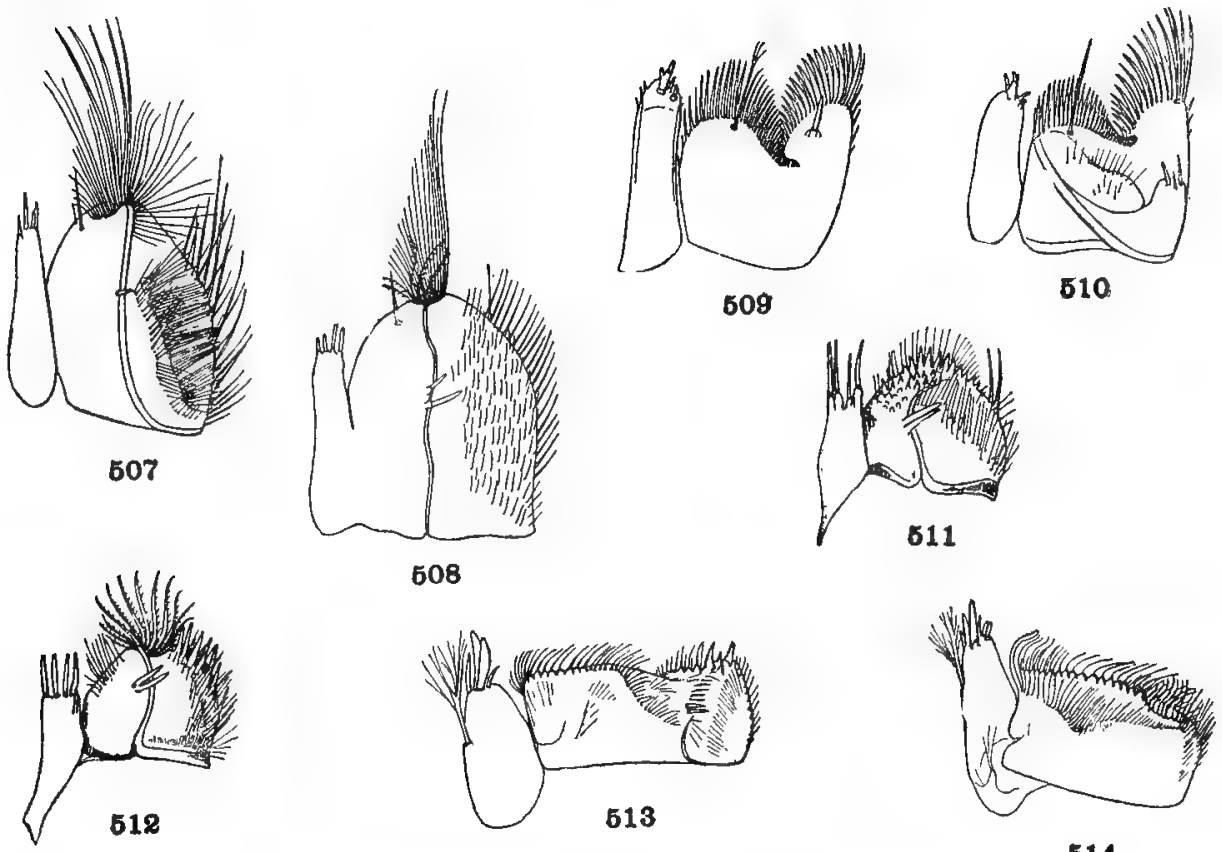

514
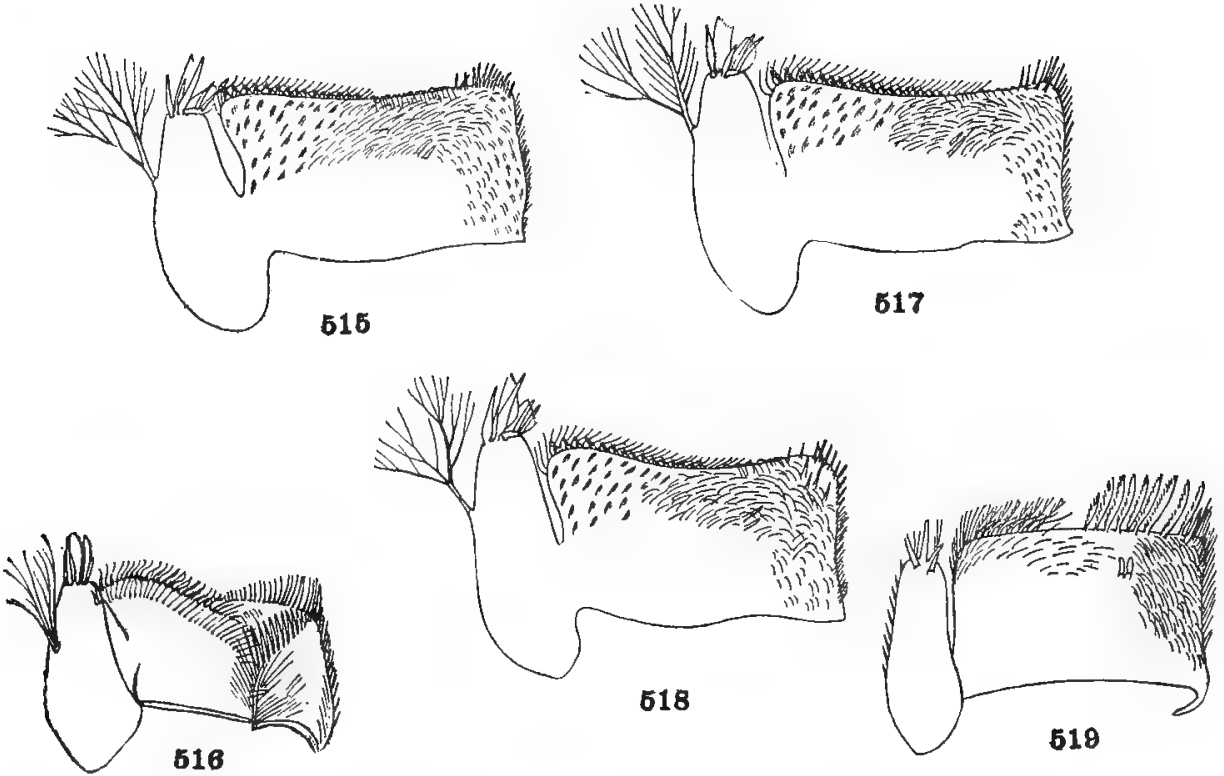

MAXILLE OF LARVA.

507. Bancroftia fascipes Coquillett.

508. Bancroftia signifer Coquillett.

509. Megarhinus septentrionalis $D$. \& $K$.

510. Megarhinus superbus Dyar \& Knab.

5II. Uranotænia geometrica Theobald.

512. Uranotænia coatzacoalcos $D$. \& $K$.
513. Anopheles eiseni Coquillett.

5I4. Anopheles grabhamii Theobald.

5I5. Anopheles punctipennis Say.

5I6. Anopheles pseudopunctipennis Theobald.

517. Anopheles crucians Wiedemann.

5I8. Anopheles quadrimaculatus Say. 


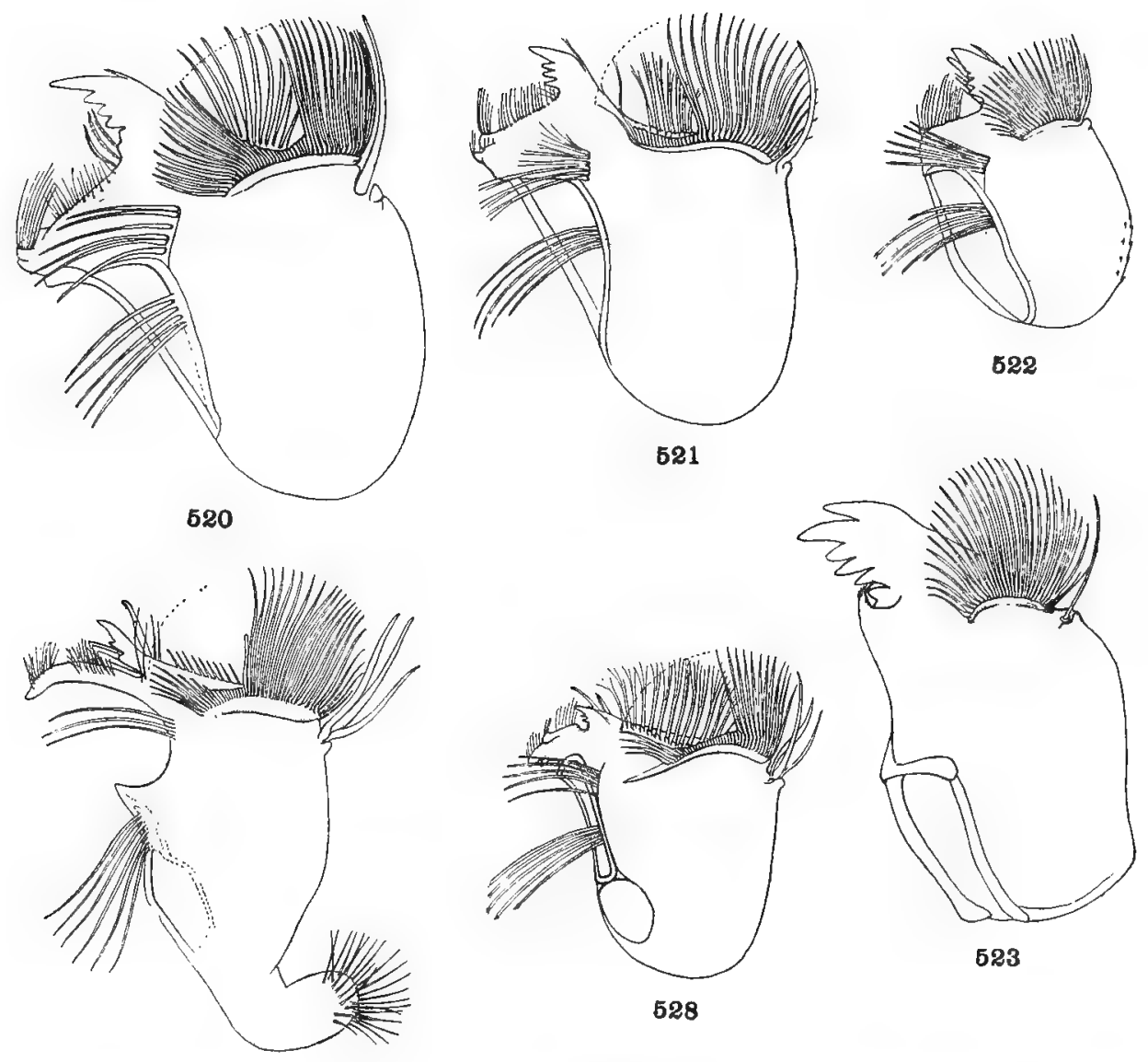

524
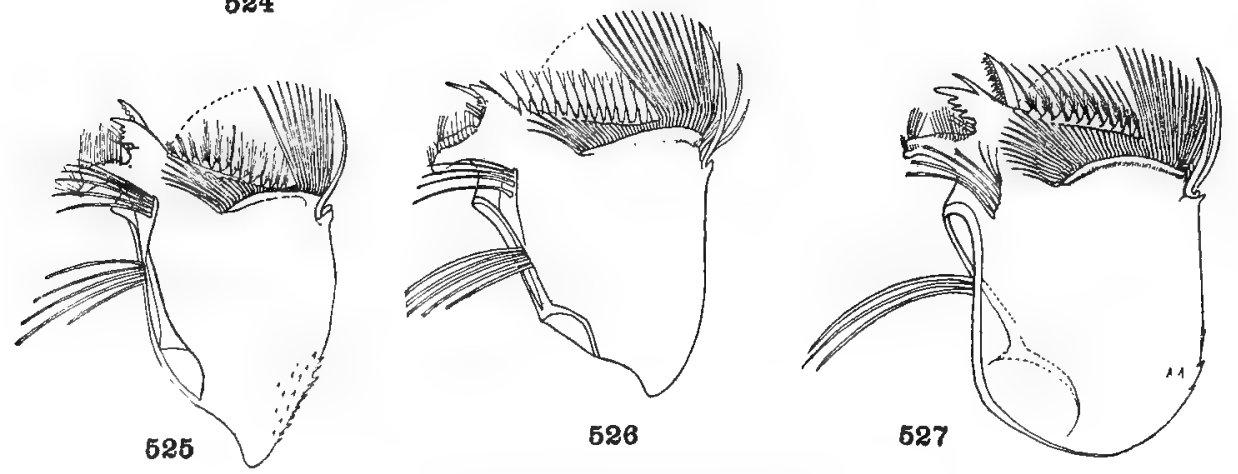

MANDIBLES OF LARVÆ.

520. Sabethinus undosus Coquillett.

521. Wyeomyia sororcula Dyar \& Knab.

522. Lesticocampa rapax Dyar \& Knab.

523. Joblotia digitatus Rondani.

524. Deinocerites cancer Theobald.

525. Culex pleuristriatus Theobald.

526. Culex rejector Dyar \& Knab.

527. Culex corniger Theobald.

528. Culex divisor Dyar \& Knab. 



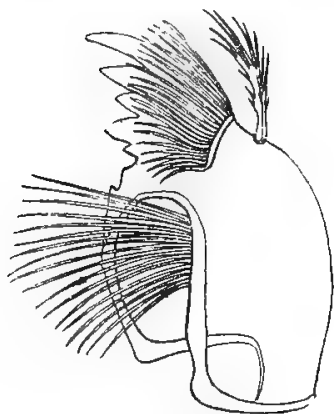

Б95

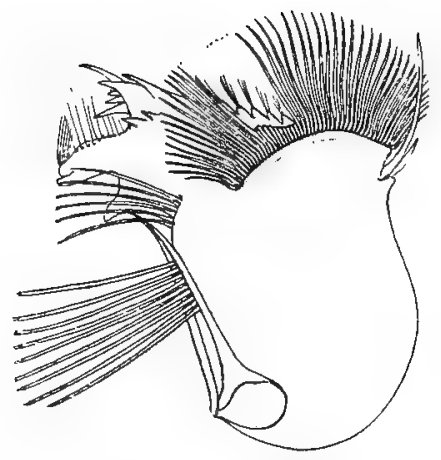

532

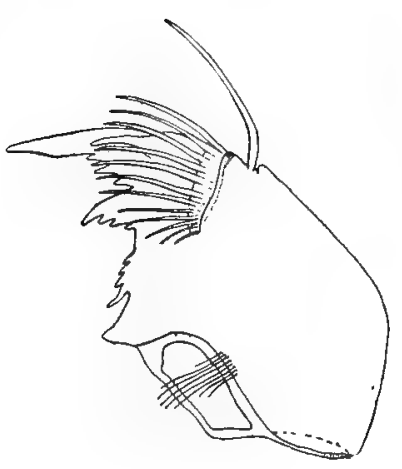

531

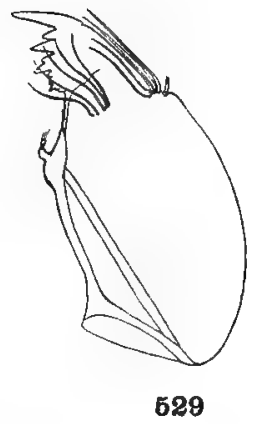

629

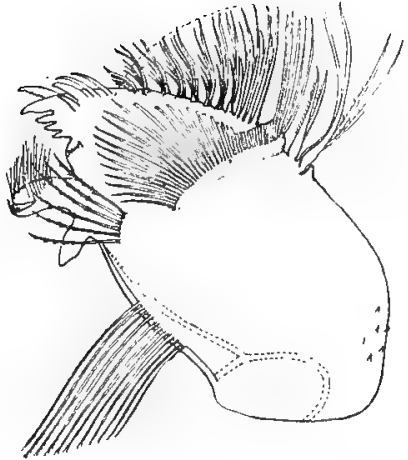

530

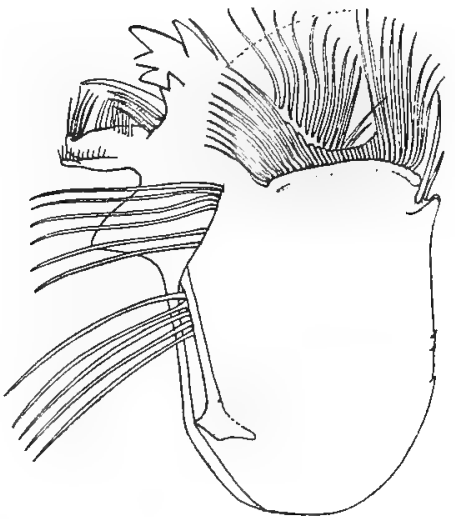

533
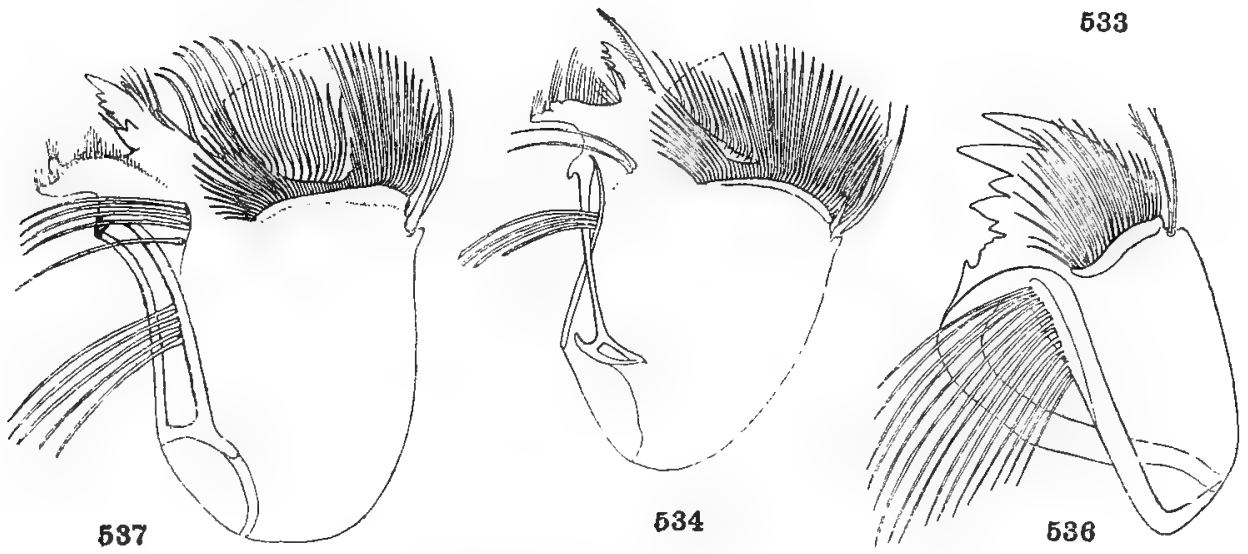

MANDIBLES OF LARVA..

529. Lutzia bigoti Bellardi.

530. Culiseta incidens Thomson.

531. Psorophora ciliata Fabricius.

532. Psorophora signipennis Coquillett.

533. Aëdes cantator Coquillett.

534. Bancroftia fascipes Coquillett.

535. Megarhinus superbus Dyar \& Knab.

536. Megarhinus septentrionalis Dyar \& $K n a b$.

537. Stegoconops lucifer Howard, Dyar \& Knab. 


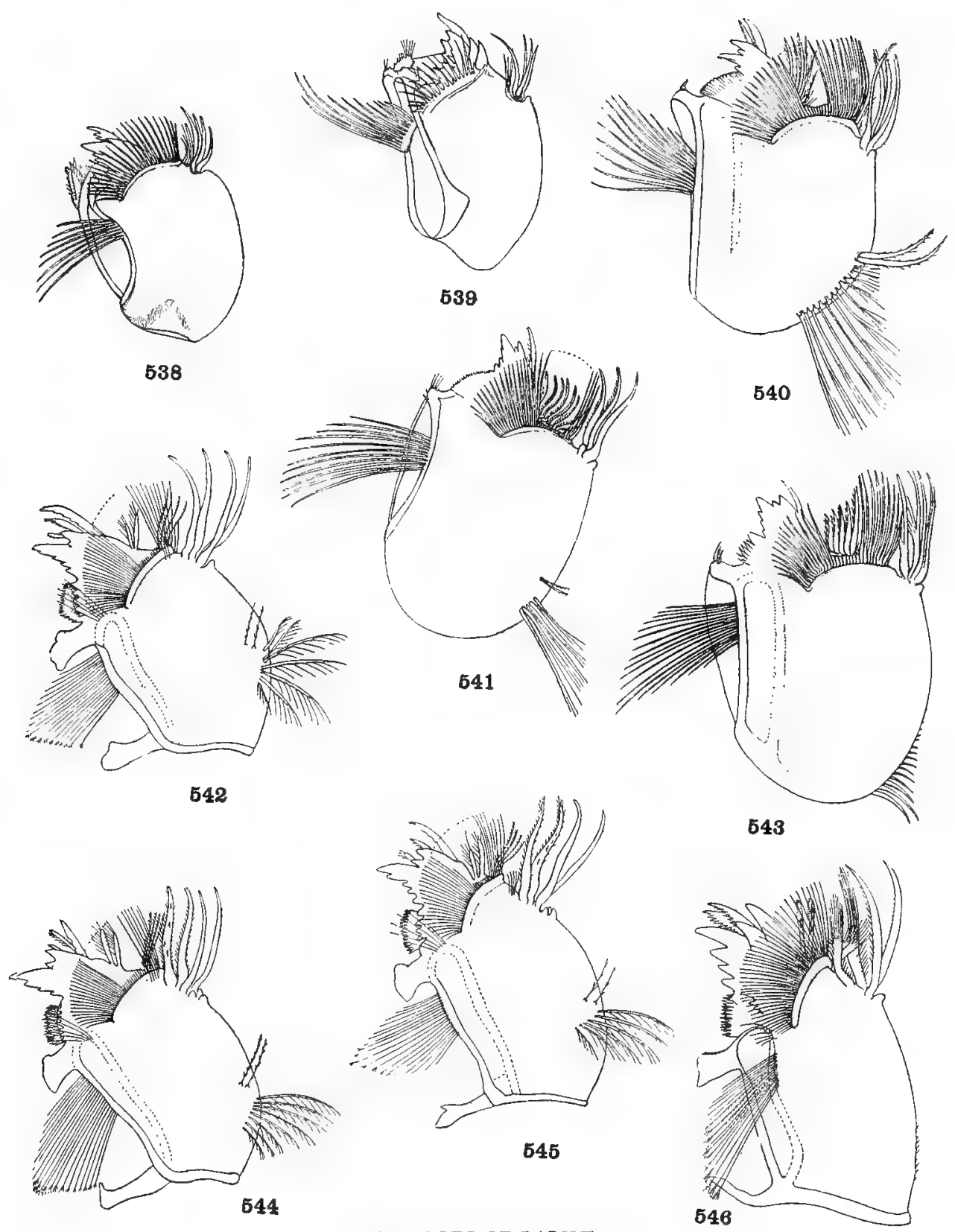

MANDIBLES OF LARVAE.

538. Uranotænia coatzacoalcos Dyar \& $K n a b$.

539. Uranotænia geometrica Theobald.

540. Anopheles grabhamii Theobald.

54I. Anopheles eiseni Coquillett.

542. Anopheles punctipennis Say.

543. Anopheles pseudopunctipennis Theobald.

544. Anopheles crucians Wiedemann.

545. Anopheles quadrimaculatus Say.

546. Cœlodiazesis barberi Coquillett. 



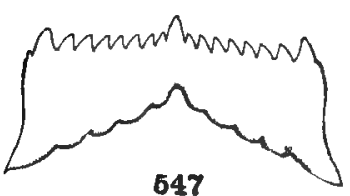

647

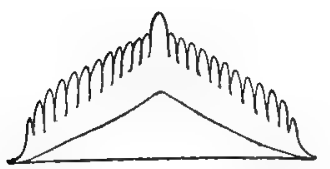

560

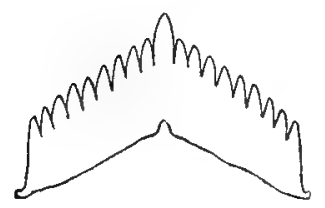

653

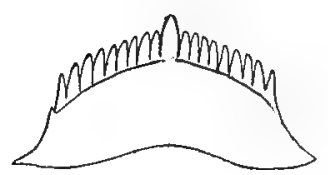

556

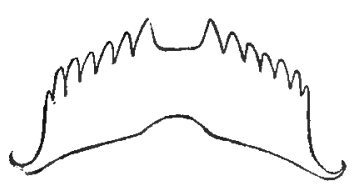

569

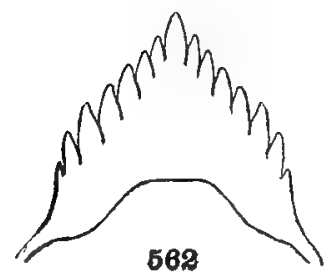

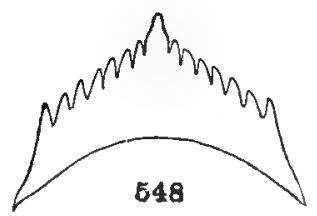

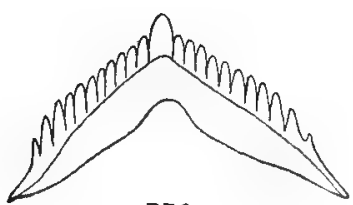

651

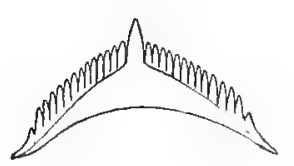

5.64

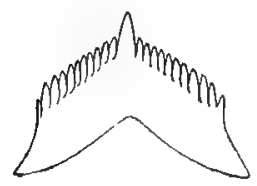

557
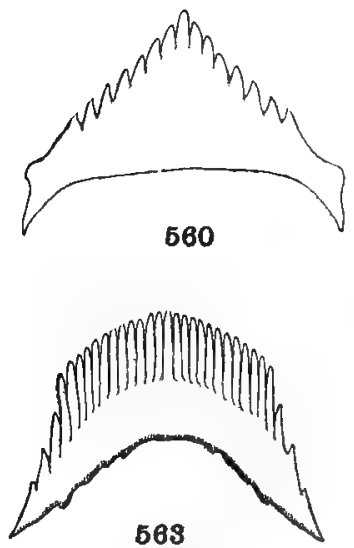

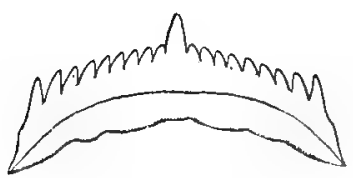

649

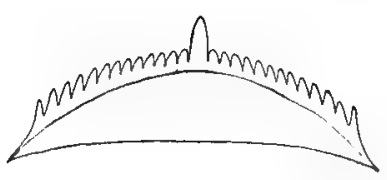

552

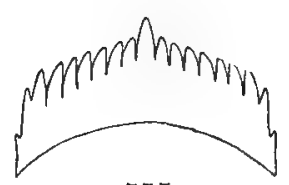

555

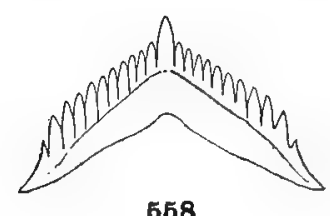

568

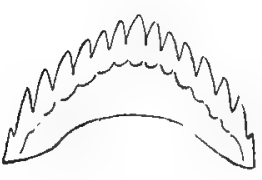

.561

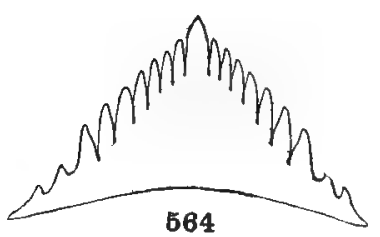

MENTAL PLATES OF LARVIE.
547. Sabethinus undosus Coquillett.

548. Limatus durhamii Theobald.

549. Wyeomyia trinidadensis Theobald.

550. Wyeomyia sororcula Dyar \& $K n a b$.

551. Wyeomyia aporonoma Dyar \& Knab.

552. Wyeomyia mitchellii Theobald.

553. Wyeomyia smithii Coquillett.

554. Wyeomyia autocratica Dyar \& Knab.

555. Wyeomyia pseudopecten $D$ yar \& $K n a b$.
556. Wyeomyia bromeliarum $D$ yar \& $K n a b$.

557. Wyeomyia telestica Dyar \& Knab.

558. Wyeomyia hemisagnosta $D$. $\mathcal{E} K$.

559. Lesticocampa rapax Dyar \& $K n a b$.

500. Joblotia digitatus Rondani.

56r. Lutzia bigoti Bellardi.

562. Culex tarsalis Coquillett.

563. Culex corniger Theobald.

564. Culex dyari Coquillet. 


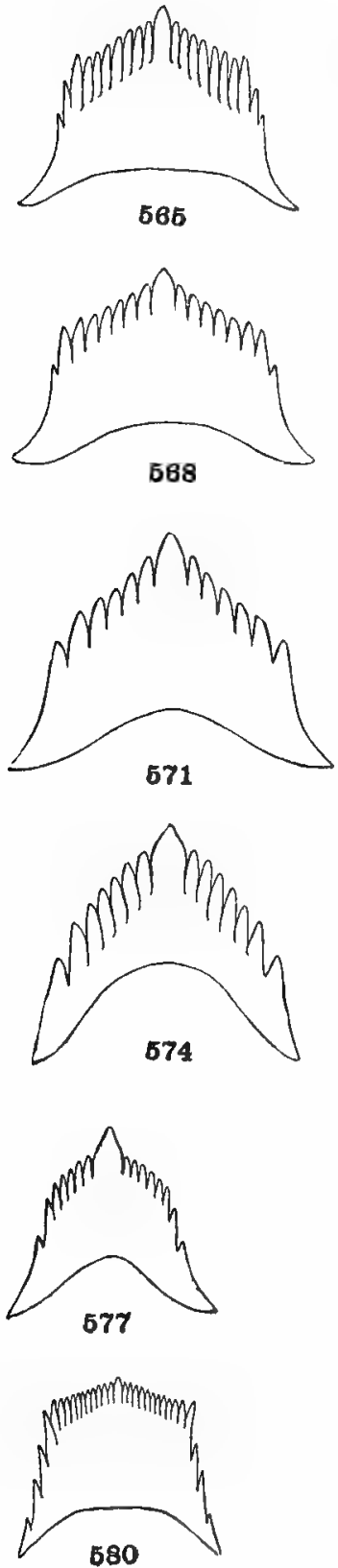
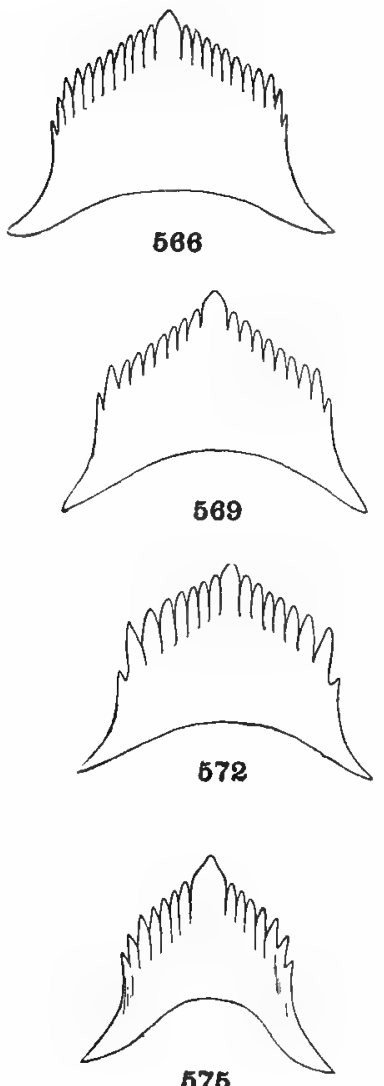

675
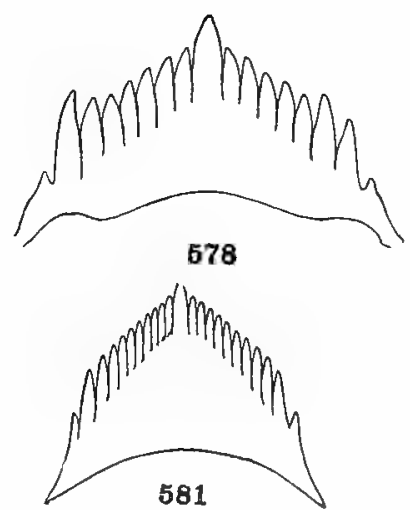

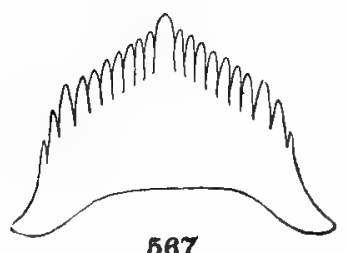

687
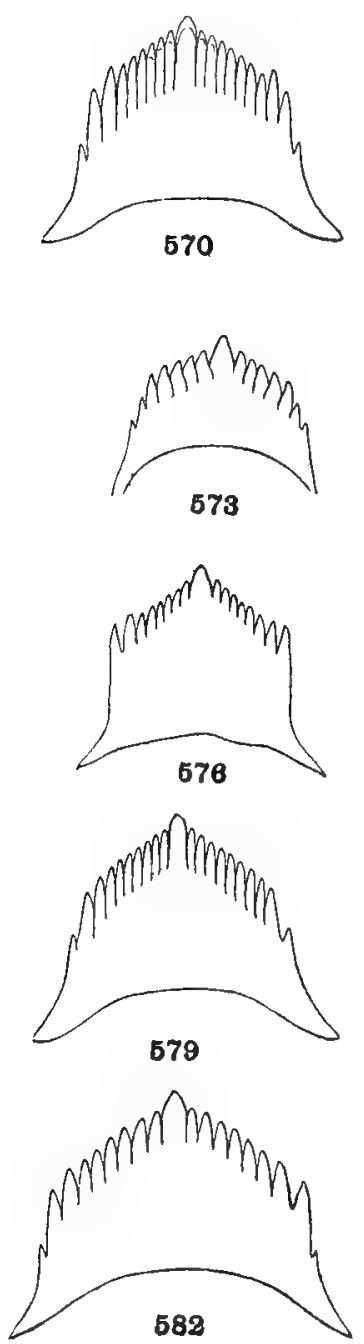

MENTAL PLATES OF LARVE.

565. Culex mortificator Dyar \& Knab.

566. Culex carmodyæ Dyar \& Knab.

567. Culex habilitator Dyar \& $K n a b$.

568. Culex declarator Dyar \& Knab.

569. Culex inquisitor $D$ yar \& $K n a b$.

570. Culex proclamator Dyar \& $K n a b$.

57r. Culex coronator Dyar \& Knab.

572. Culex derivator Dyar \& $K n a b$.

573. Culex territans $W$ alker.
574. Culex bahamensis Dyar \& Knab. 575. Culex simulator Dyar \& Knab. 576. Culex bisulcatus Coquillett. 577. Culex conservator Dyar \& Knab. 578. Culex melanurus Coquillett. 579. Culex extricator Dyar \& Knab. 580. Culex restuans Theobald. 581. Culex barbarus Dyar \& Knab. 582. Culex similis Theobald. 



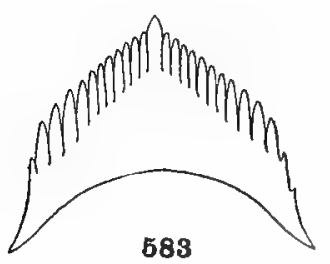

583

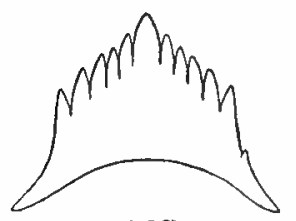

587

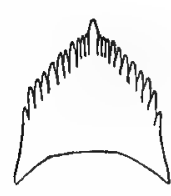

584
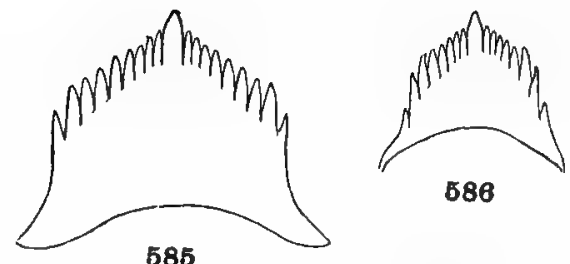

588

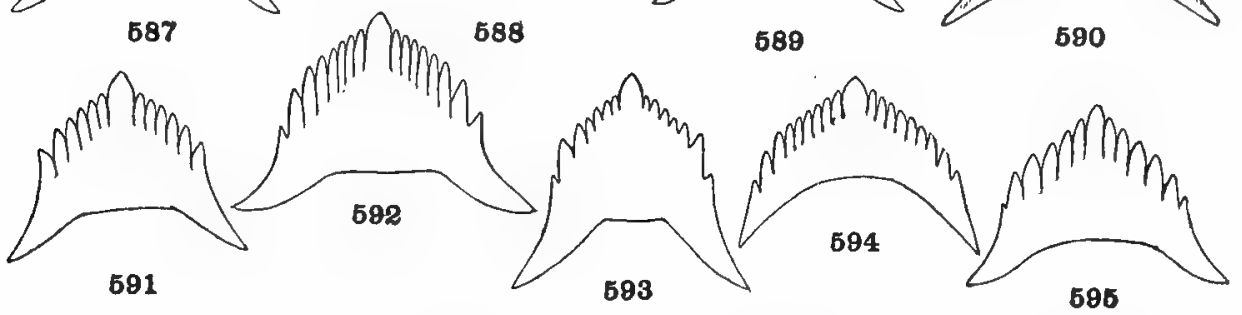

593

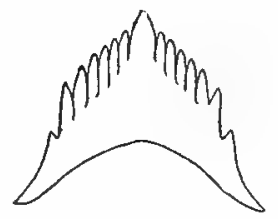

688

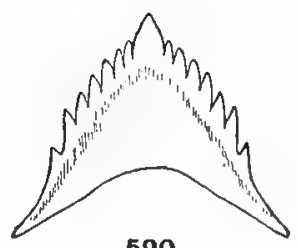

590

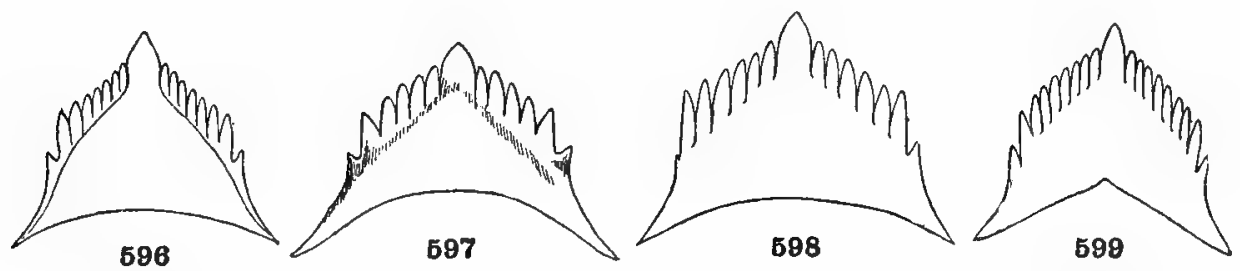

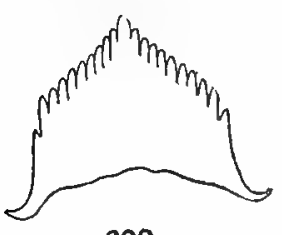

600

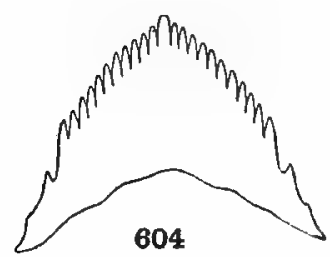

604

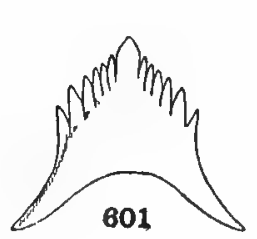

601

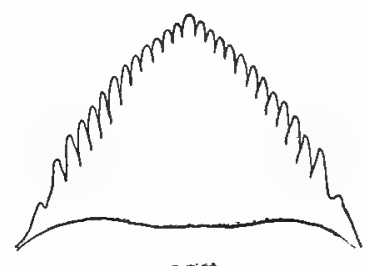

605

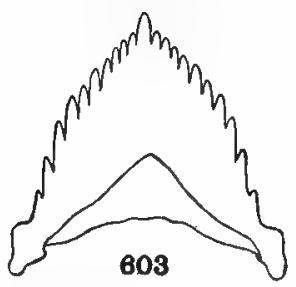

MENTAL PLATES OF LARVÆ.

583. Culex quinquefasciatus Say.

584. Culex pipiens Linnous.

585. Culex factor Dyar \& $K n a b$.

586. Culex salinarius Coquillett.

587. Culex investigator Dyar \& $K n a b$.

588. Culex erraticus Dyar \& $K n a b$.

589. Culex agitator Dyar \& $K n a b$.

590. Culex caudelli Dyar \& Knab.

591. Culex conspirator Dyar \& Knab.

592. Culex carcinophilus Dyar \& $K n a b$.

593. Culex elevator Dyar \& $K u a b$.

594. Culex interrogator Dyar \& $K n a b$.
595. Culex mutator Dyar \& Knab.

596. Culex restrictor Dyar \& Knab.

597. Culex decorator Dyar \& $K n a b$.

598. Culex imitator Theobald.

599. Culex rejector Dyar $\mathcal{E} K n a b$.

600. Culex pleuristriatus Theobald.

6or. Culex gravitator Dyar \& $K n a b$.

602. Culex inimitabilis Dyar \& $K n a b$.

603. Carrollia urichii Coquillett.

604. Culiseta incidens Thomson.

605. Culiseta inornatus Williston.

606. Psorophora ciliata Fabricius. 



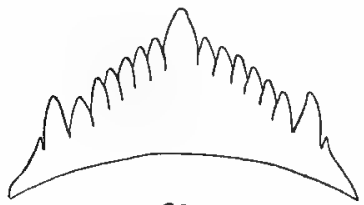

607

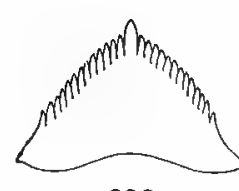

608

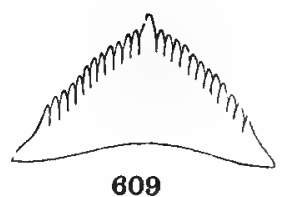

609

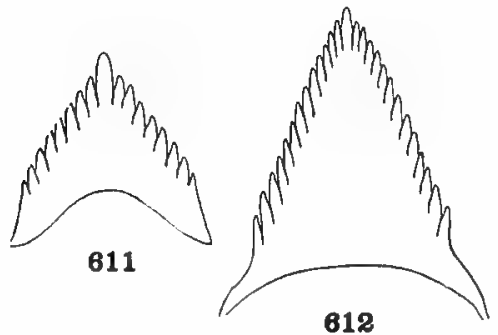

812

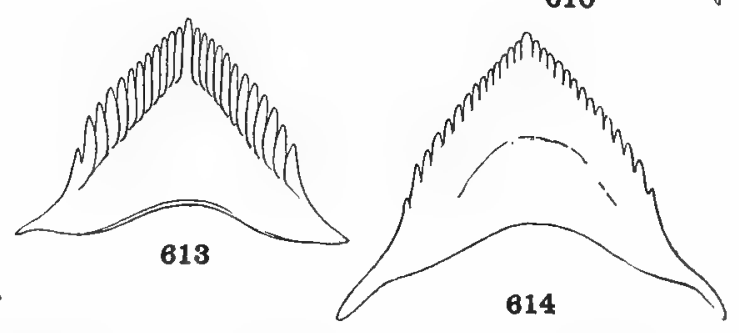

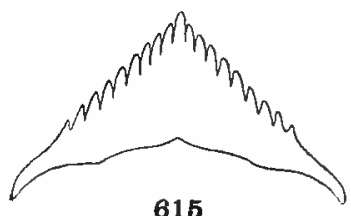

615

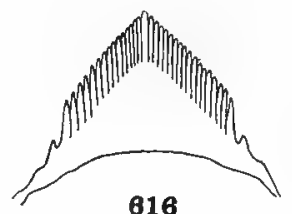

816

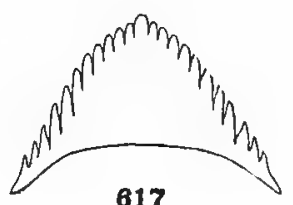

617
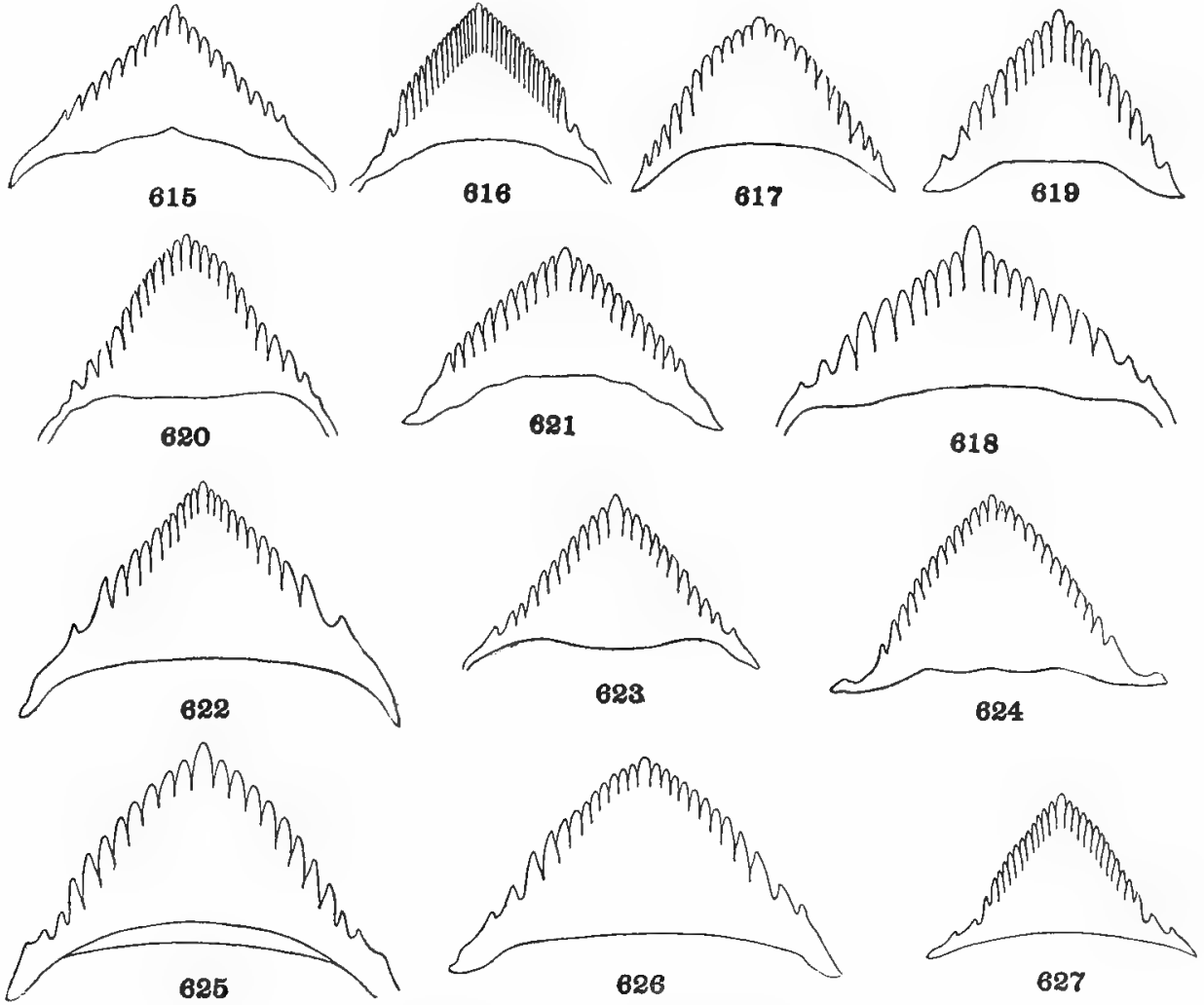

MENTAL PLATES OF LARVE.

607. Psorophora howardii Coquillett.

608. Psorophora sayi Dyar \& Knab.

609. Psorophora discrucians Walker.

6ro. Psorophora signipennis Coquillett.

6rI. Psorophora discolor Coquillett.

6r2. Psorophora columbix Dyar $\mathcal{E} K n a b$.

6r3. Psorophora infine Dyar \& $K n a b$.

6I4. Psorophora indoctum Dyar \& $K n a b$.

6I5. Psorophora insularius Dyar \& $K n a b$.

6I6. Aëdes bimaculatus Coquillett. 6r7. Aëdes curriei Coquillett.

6I8. Aëdes atropalpus Coquillett.

6rg. Aëdes varipalpus Coquillett.

620. Aëdes canadensis Theobald.

621. Aëdes sollicitans Walker.

622. Aëdes mitchella Dyar.

623. Aëdes tæniorhynchus Wiedemann.

624. Aëdes stimulans Walker.

625. Aëdes fitchii Felt \& Young.

626. Aëdes abfitchii Felt.

627. Aëdes sylvestris Theobald. 


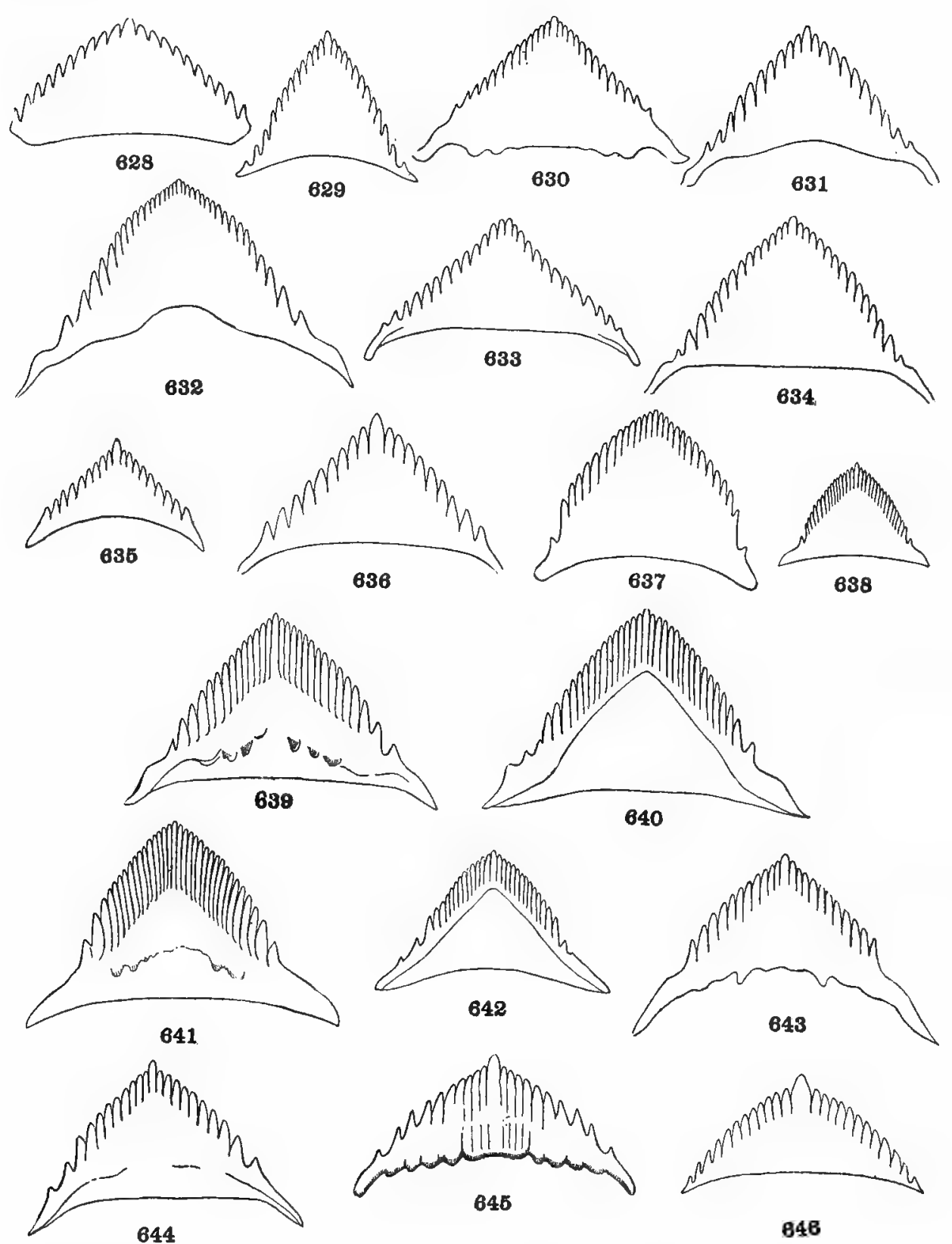

848

MENTAL PLATES OF LARV $Æ$.

628. Aëdes cantator Coquillett.

629. Aëdes fuscus Osten Sacken.

630. Aëdes lazarensis Felt $\mathcal{E}$ Young.

631. Aëdes pullatus Coquillett.

632. Aëdes hirsuteron Theobald.

633. Aëdes auroides Felt.

634. Aëdes trichurus Dyar.

635. Aëdes triseriatus Say.

636. Aëdes aurifer Coquillett.
637. Aëdes trivittatus Coquillett.

638. Aëdes dupreei Coquillett.

639. Aëdes euplocamus Dyar \& $K n a b$.

640. Aëdes serratus Theobald.

641. Aëdes atlanticus Dyar \& $K n a b$.

642. Aëdes bracteatus Coquillett.

643. Aëdes podographicus Dyar \& $K n a b$.

644. Aëdes oswaldi Lutz.

645. Aëdes mediovittata Coquillett. 



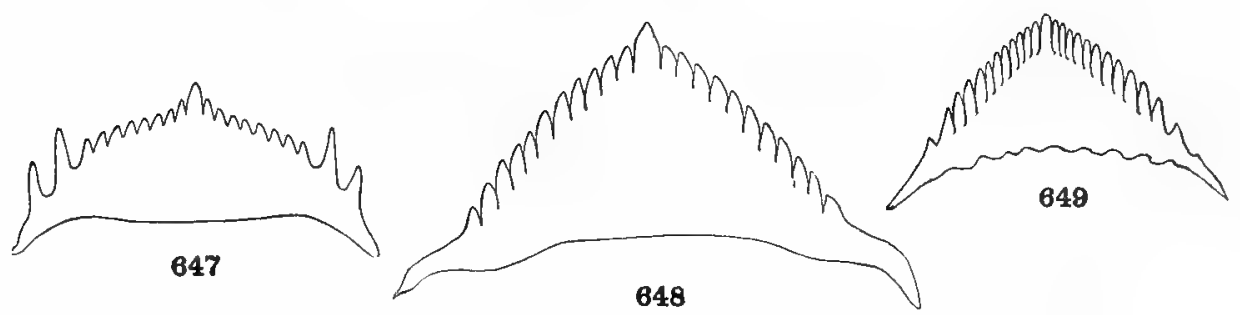

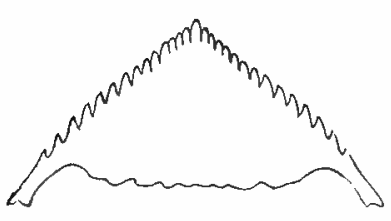

650
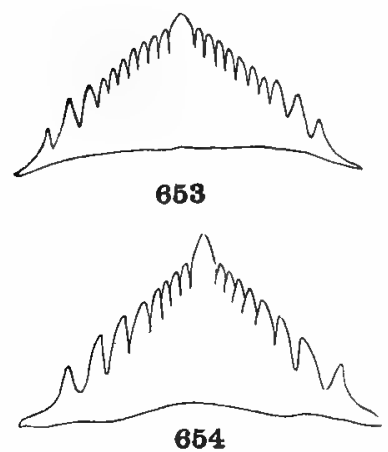

654

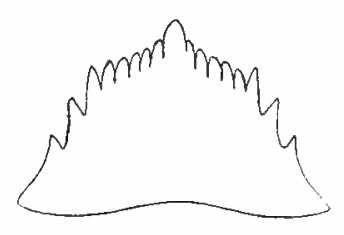

651
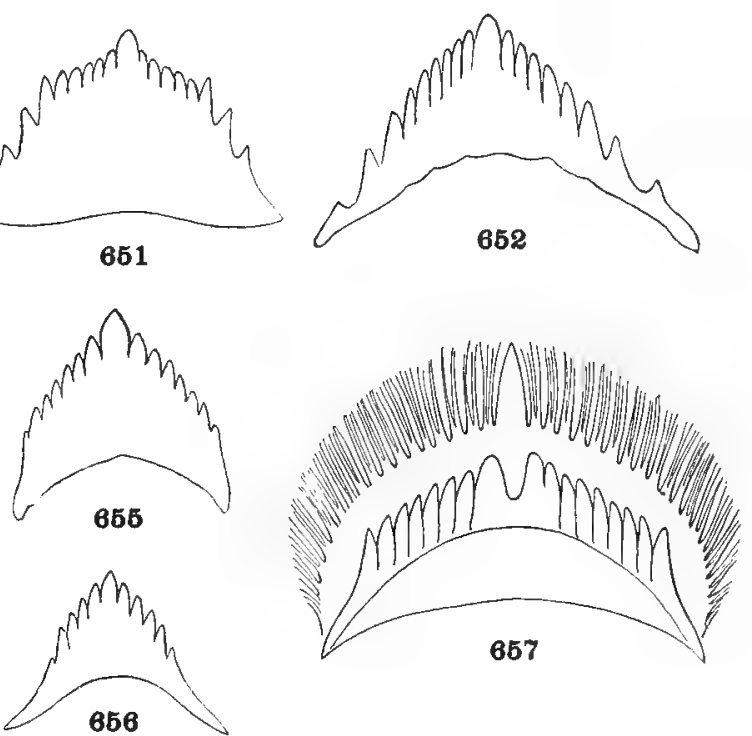

656

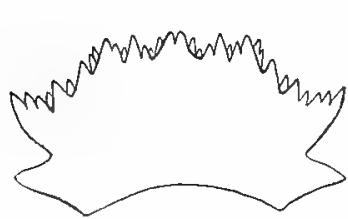

658

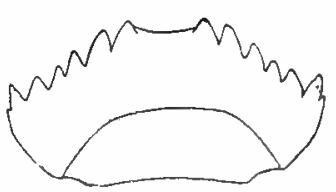

659
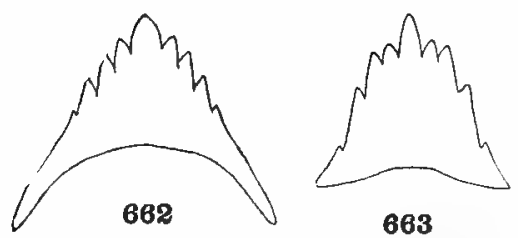

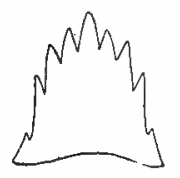

664

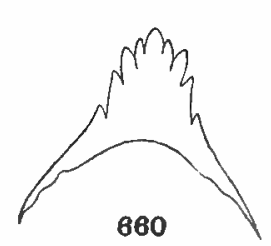

680

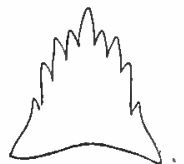

665

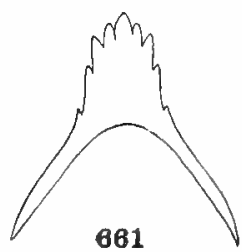

661

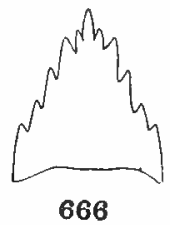

666

MENTAL PLATES OF LARVA.

647. Aëdes knabi Coquillett.

648. Aëdes walkeri Theobald.

649. Aëdes albonotata Coquillett.

650. Aëdes busckii Coquillett.

65I. Stegoconops lucifer $H ., D$. \& $K$.

652. Stegoconops equinus Theobald.

653. Bancroftia fascipes Coquillett.

654. Bancroftia signifer Coquillett.

655. Uranotrenia geometrica Theobald.

656. Uranotænia coatzacoalcos $D$. $\mathcal{F} K$.
657. Megarhinus septentrionalis $D$. \& $K$. 658. The same, second plate.

659. The same, third plate.

660. Anopheles grabhamii Theobald.

66I. Anopheles eiseni Coquillett.

662. Anopheles pseudopunctipennis Theob.

663. Anopheles crucians Wiedemann.

664. Anopheles punctipennis Say.

665. Anopheles quadrimaculatus Say.

666. Colodiazesis barberi Coquillett. 



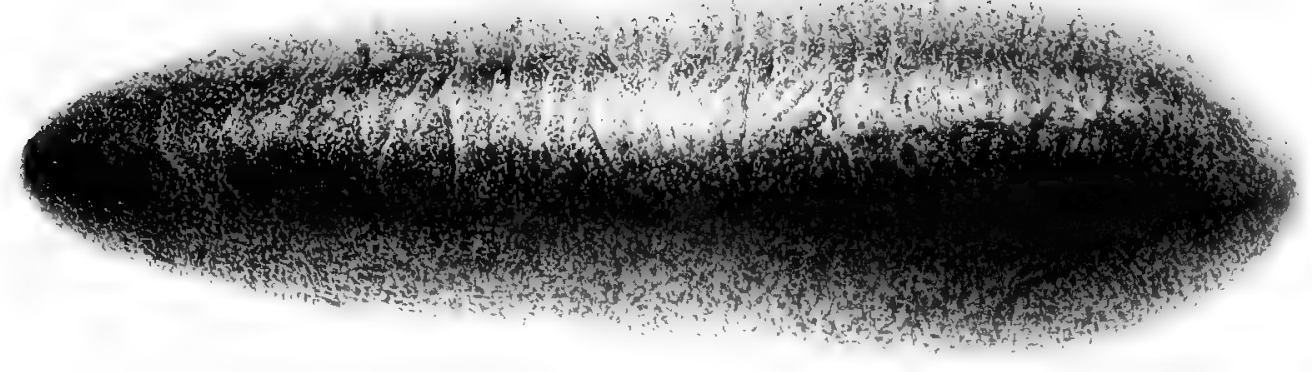

\section{7}

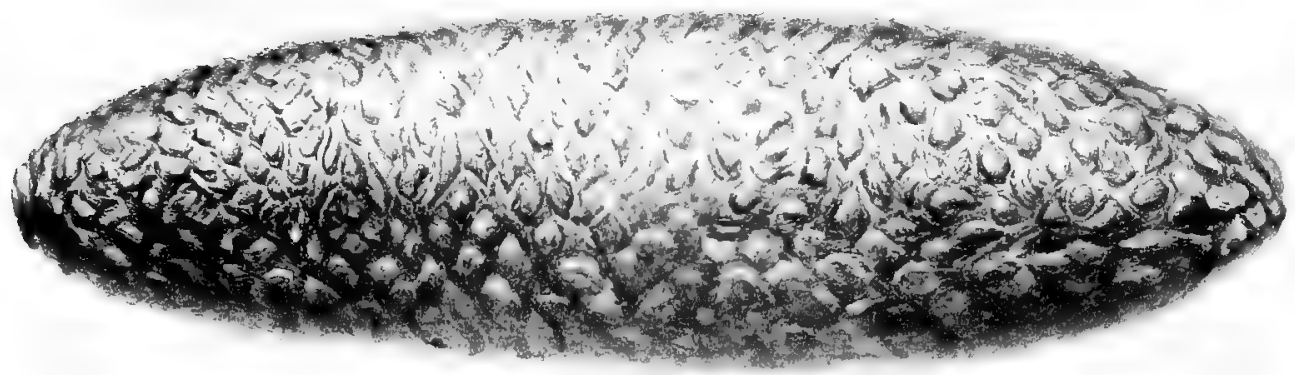

668

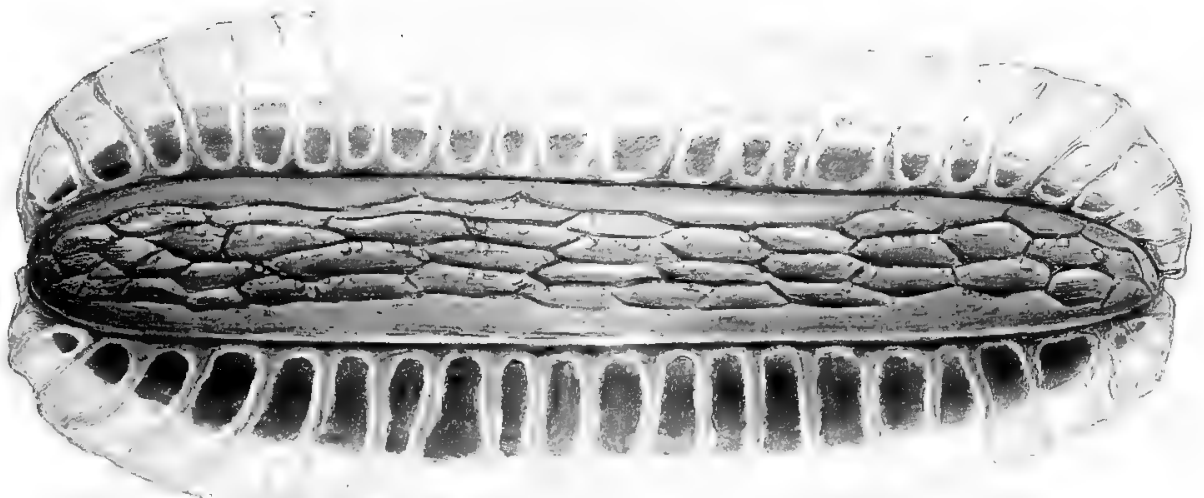

669

Eggs:

667. Aedes atropalpus Coquillett.

668. Aedes calopus Meigen.

669. Bancroftia signifer Coquillett. 


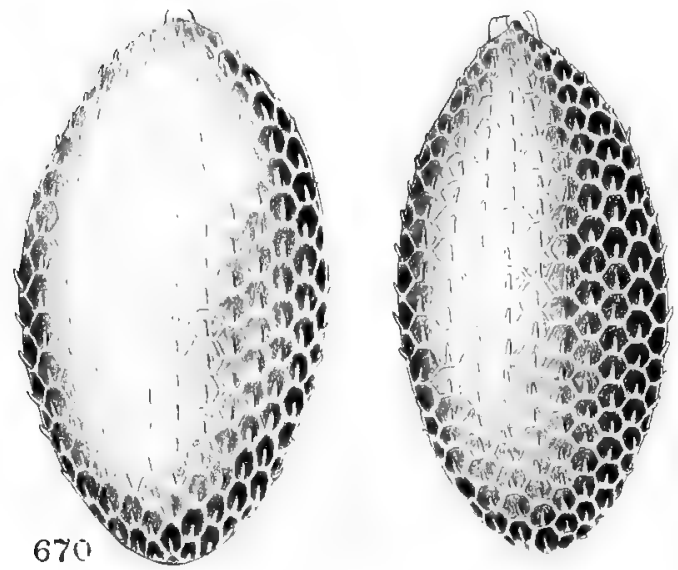

671

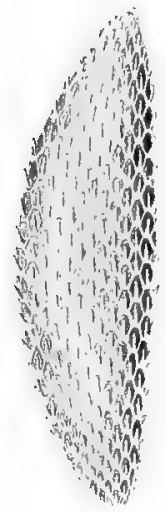

675

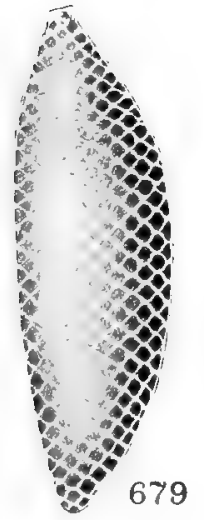

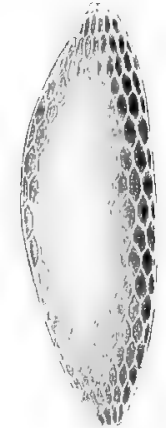

677

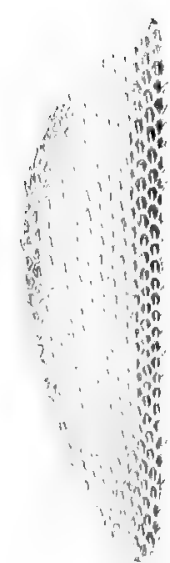

672

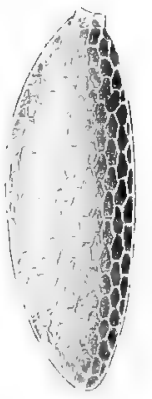

678

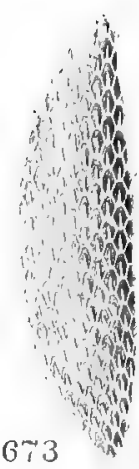

674
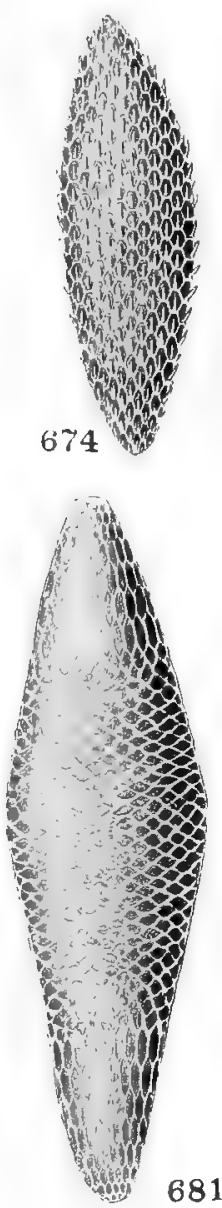

\section{Eggs :}

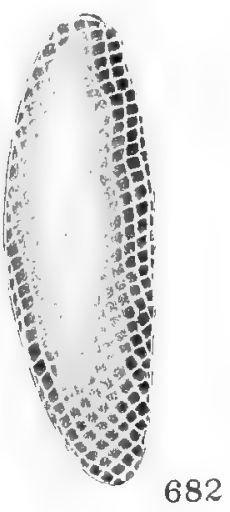

6i6. Aedes triseriatus Say.

67\%. Aedes sylvestris Theobald.

678. Aedes tæniorhynchus Wiedemann.

679. Aedes sollicitans Walker.

680. Aedes infirmatus Dyar \& Knab.

681. Aedes tormentor Dyar \& Knab.

682. Aedes canadensis Theobald. 



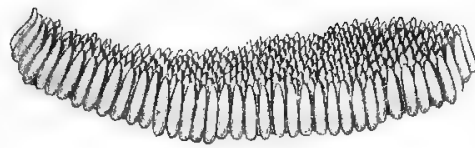

686
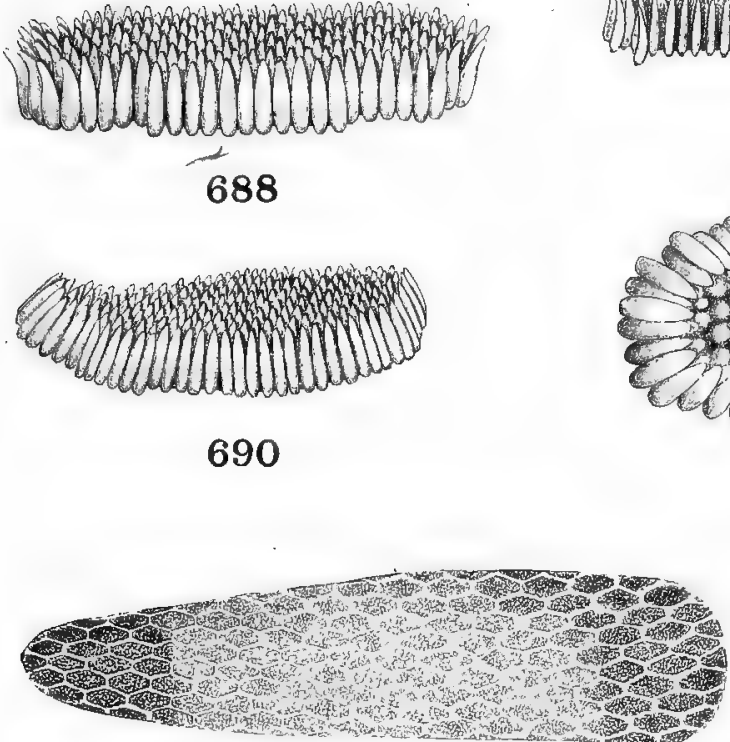

692

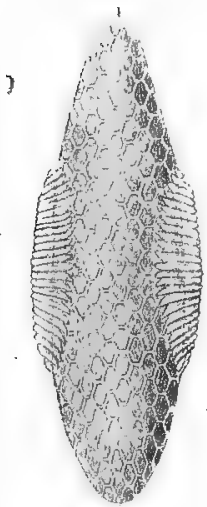

693

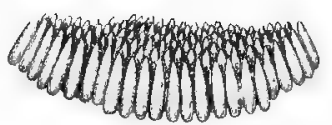

687

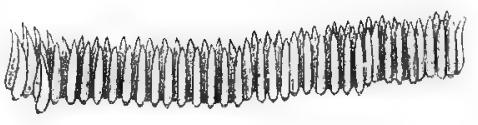

689

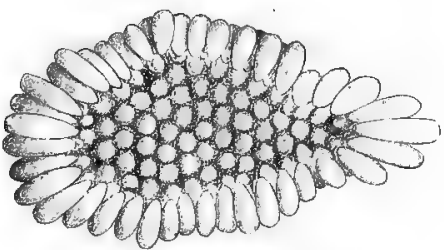

683

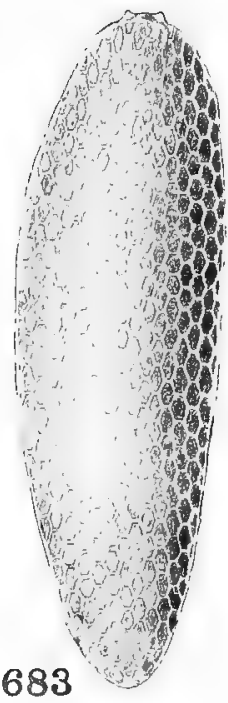

691

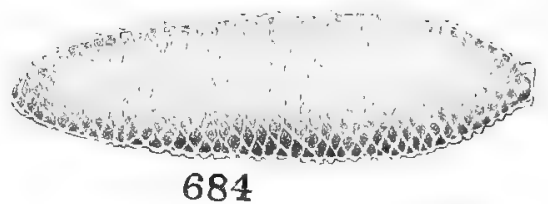

684

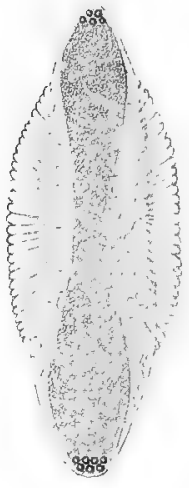

696

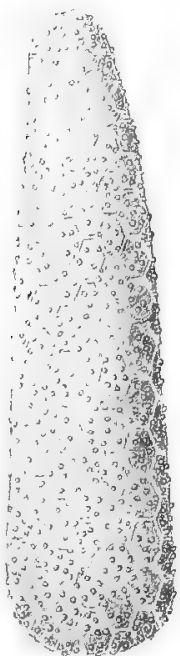

685

Eggs :

683. Aedes grossbecki Dyar \& Knab.

684. Aedes calopus Meigen.

685. Mansonia perturbans Walker

686. Culex pipiens Linncus.

687. Culex salinarius Coquillett.

688. Culex territans Walker.

689. Culex erraticus Dyar \& Kuab.
690. Culex restuans Theobald.

691. Culiseta inornatus Williston.

692. The same, single egg.

693. Anopheles punctipennis Say, dorsal view.

694. The same, ventral view. chorsal

695. Anopheles quadrimaculatus Say, view.

696. Anopheles crucians Wiedemann, ventral view. 


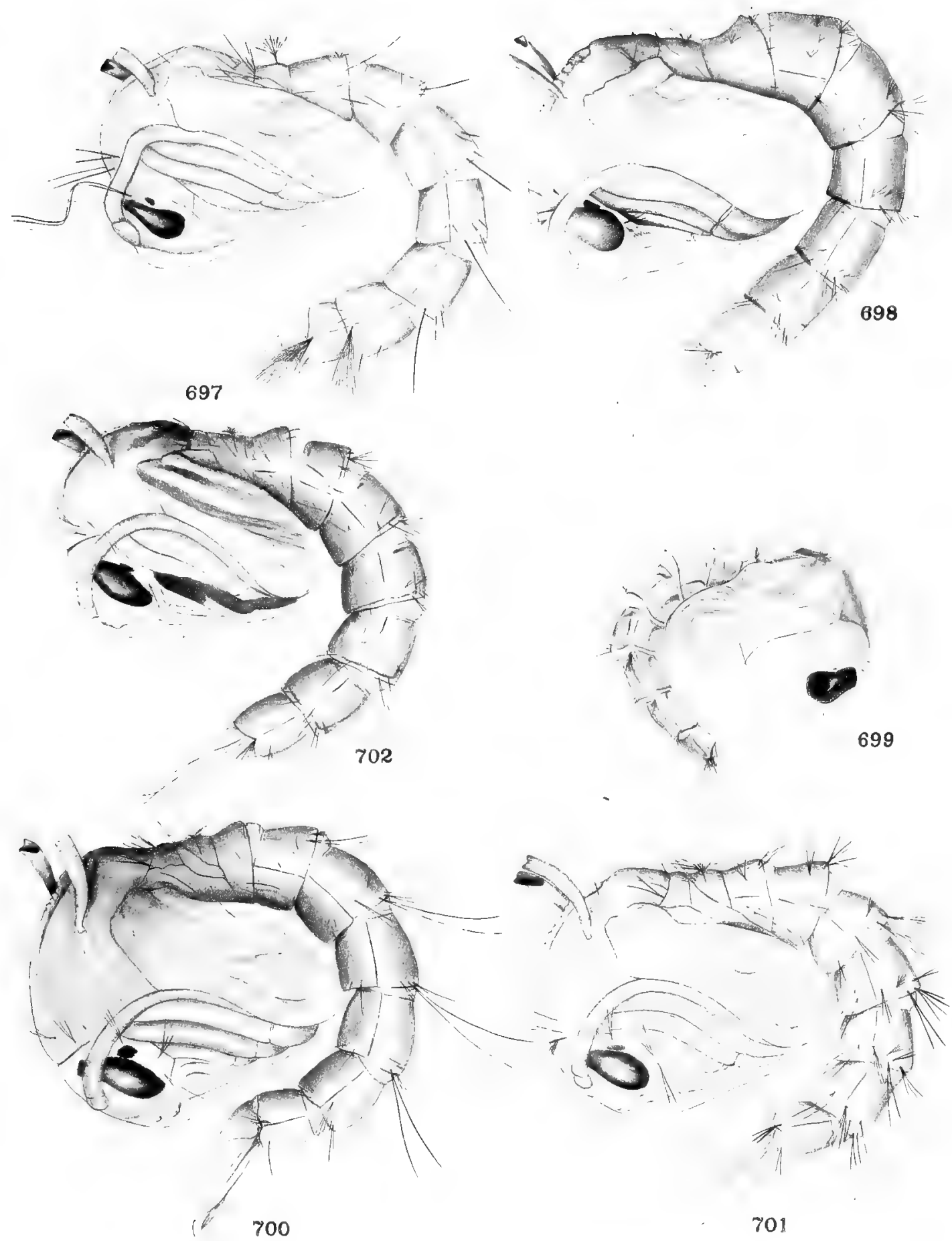

Pupæ:

697. Wyeomyia smithii Coquillett.

698. Culex territans Walker.

699. Wyeomyia circumcincta Dyar \& Knab.
700. Culex restuans Theobald.

701. Culex pipiens Linnaus.

702. Aedes sylvestris Theobald. 



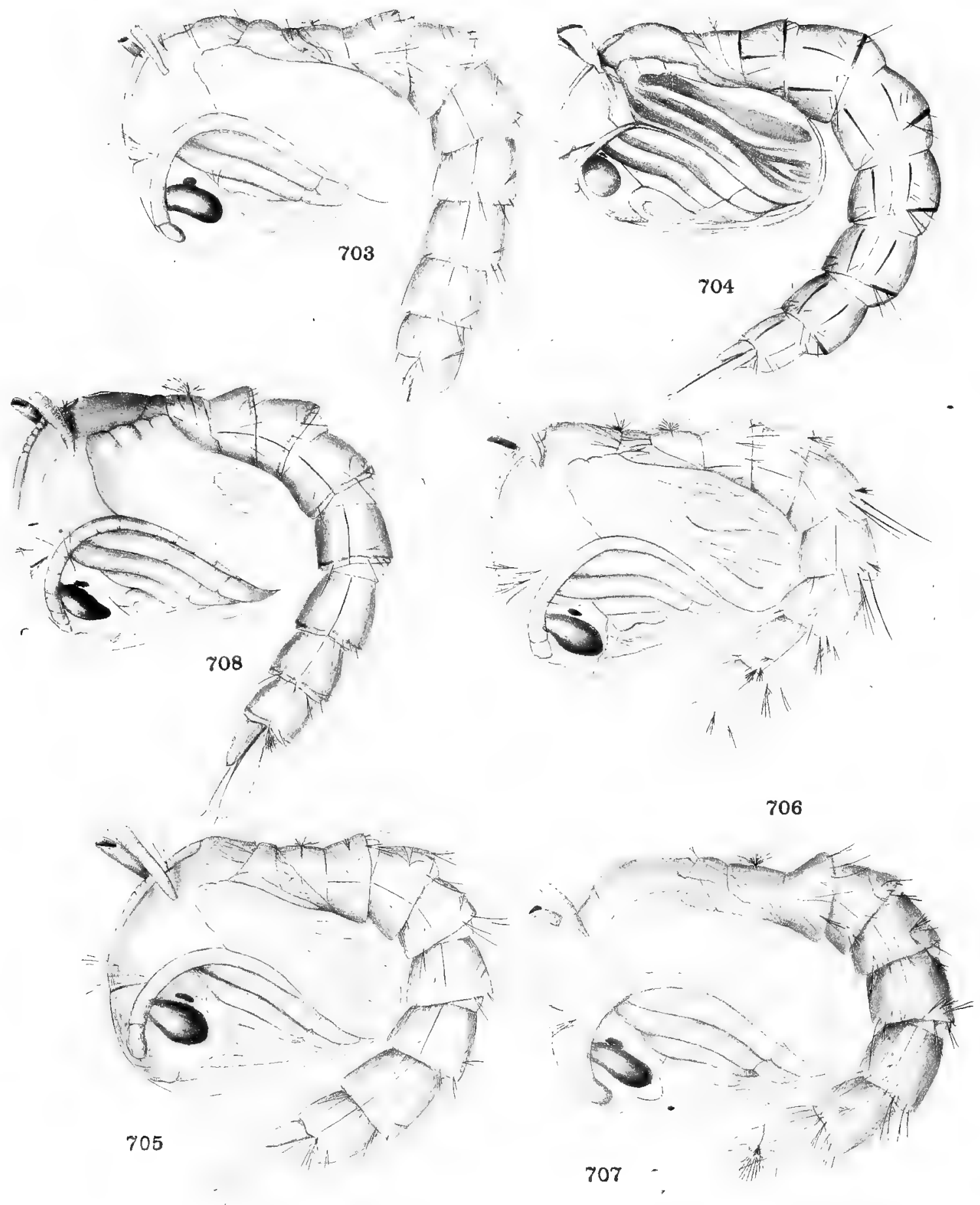

Pupæ:

703. Aedes fuscus Osten Sacken.

704. Psorophora ciliata Fabricius.

705. Psorophora sayi Dyar \& Knab.
706. Psorophora columbix Dyar \& Knab.

70\%. Bancroftia signifer Coquillett.

708. Aedes triseriatus Say. 


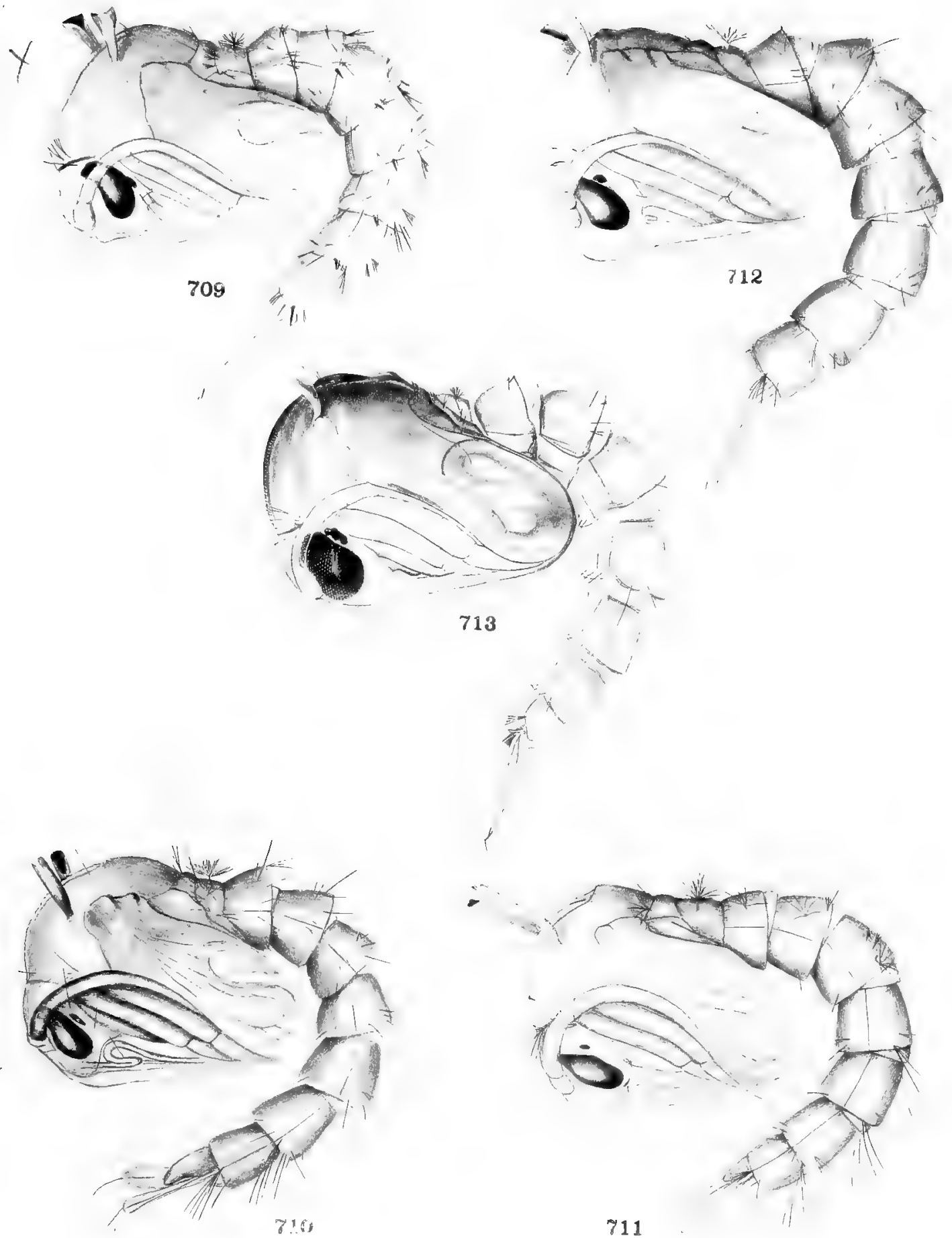

Pupæ:

709. Anopheles punctipennis Say.

710. Aedes mediovittata Coquillett.
711. Uranotænia sapphirinus Osten Sacken.

712. Aedes atropalpus Coquillett. 






